\title{
RADIATION LOSSES IN \\ DIELECTRIC OPTICAL WAVEGUIDES
}

\author{
Ian Arthur White
}

A thesis submitted for the degree of Doctor of Philosophy

at the Australian National University, Canberra

August 1977 


\section{PREFACE}

This dissertation is an account of work carried out in the Department of Applied Mathematics of the Institute of Advanced Studies in the Australian National University between March 1974 and August 1977 under the supervision of Drs. Allan w. Snyder and Colin Pask.

While I have benefited immeasurably throughout this time from discussions with members of this Department and other workers in the field, the work described in the following pages is, unless explicitly disowned in the text, my own.

None of this work has been submitted to any other institution of learning for any degree.

$$
\text { San A. White. }
$$

I.A. White 


\section{PUBLICATIONS}

1. A.W. Snyder, I.A. White and D.J. Mitchell

Radiation from bent optical waveguides

Electronics Letters 11, no. 15 (1975) 332.

2. I.A. White

Radiation from dielectric optical fibres

Presented at 1976 R.R.B. Symposium "Optics Communications and Integrated Optics", Melbourne 1976).

3. I.A. White, A.W. Snyder and C. Pask

Directional change of beams undergoing partial reflection Joumal of the Optical Society of America 67 (1977), 703.

4. I.A. White and A.W. Snyder

Radiation from dielectric optical fibres - a comparison of techniques

Applied Optics

5. B.J. Dalton and I.A. White

Neoclassical theory of resonance scattering from certain atomic systems

Journal of Quantum Spectroscopy and Radiative Transitions

6. I.A. White and C. Pask

Goos-Hänchen shifts in optical fibres

Applied Optics

7. I.A. White

Reflection of cylindrical waves from concentric cylinders

[in preparation]

8. I.A. White

Radiation from dielectric optical fibres: a correction to the Volume Current Method

[in preparation]

9. A.W. Snyder and I.A. White

Radiation from dielectric optical fibres

[in preparation]

10. I.A. White

Ray optics interpretation of field deformation and mode coupling due to bends in optical waveguides

[in preparation] 
11. I.A. White

Curvature induced radiation losses in graded-index fibres [in preparation]

12. I.A. White

Lateral shift of a beam incident upon a parabolic dielectric halfspace

[in preparation] 


\section{ACKNOWLEDGEMENTS}

Before beginning with the acknowledgements, I would like to apologise for the obesity of this thesis. The reader should also consider all the imperfections of this thesis on imperfections in optical waveguides prosaic rather than mechanistic or intellectual blundering!

I have been exceptionally lucky to have worked in the Department of Applied Mathematics, with the humour, enthusiasm and friendship of the staff, students and workers making it a most relaxed environment in which to work. The small size and geographic location of the Department together with the high quality of the staff and students over a diversity of disciplines have allowed for the best possible interactions between Department members. The heated, but good hearted, arguments that are continually featuring in the Departmental tea room provide an excellent forum in which to unashamedly iron out one's ideas on anything from the eightfold way to most appropriate dietary procedures!

In particular, I would like to thank Barry Ninham, erstwhile Head of the Department, for his enthusiasm for science and his awe inspiring pipe performance upon my introduction to the Department. His continuing friendship and the hyperactivity and conceptual fecuntity of one Allan Whitenack Snyder have rekindled my enthusiasm for science and humanity. Thanks must also go to my other supervisor Colin Pask and to John Mitchell, without whose moral and intellectual support, none of this would be possible. For the long, heated and uncompromising arguments with John Love, I thank him for his tolerance.

During the three and one-half years taken to complete this tome, I have been privileged to meet many well respected research 
workers in electromagnetic theory and colloid science who have visited the Department. Particular thanks must go to Dr. Dieter Marcuse of Bell Labs., U.S.A., with whom I spent many enjoyable hours trying to appreciate Coupled Mode Theory. From this brief personal exposure and our continuing correspondence, I have obtained much perspective in the field of fibre optics and in particular the idea for Chapter 4 of this thesis owes much to our discussions.

The students of the Department have provided me with many interesting ideas, some connected with science, and in particular I should like to thank Kevin Kavanagh (née Barrell) for some of the computational analysis of this thesis.

The greatest accolades must go to Norma Chin, the magnificent lady who managed to transfer my voluminous illegible script into this visually appealing tome, with the minimum of physical violence to my person. Her advice and threats have been a strong motivation for the completion of this thesis and the quality of the presentation. Thanks also to Pauline Wallace and Diane Shepherd for tolerating me and for typing my manuscripts throughout the duration of my thesis.

I would like to thank my brother Lee and his lovely wife Yvonne and my niece Emma and nephew Jamie, for providing an island of sanity and humour in the midst of my trials and tribulations. To all the above and especially to all the beautiful women in the world without whose inspiration none of this would be possible, I express my sincerest thanks.

Finally, I am grateful for financial support from a Commonwealth Post-graduate Award to undertake this study. 


\section{ABSTRACT}

The effect of irregularities and deviations from the perfect structure of the ideal waveguide is to scatter some of the guided power carried by the modes of the ideal waveguide incident on the irregularity. This scattered power is redistributed over the (non-attenuating) bound modes of the structure and into the radiation modes of the waveguide. The study of this redistribution of the power over the discrete (bound) mode spectrum can be adequately analysed by conventional electromagnetic Coupled Mode Theory. However, the application of this technique for the analysis of the radiative losses, i.e. the coupling into the radiation modes of the waveguide proves to be extremely tedious due to the difficulty of normalisation and orthogonalisation of these "improper" modes. The aim of this thesis is to present alternative techniques to the Coupled Mode Theory analysis, which provide simple treatments of these radiation loss processes in weakly guiding dielectric optical fibres. The philosophy of the presentation is the present the technique and choose the least number of practical examples that elucidate the strengths and deficiencies of each approach, rather than list ad nauseum a wide range of practical examples.

In Chapter 1, a general background to the theoretical analysis of propagation in dielectric optical waveguides is presented, together with a qualitative introduction to the effects of irregularities and their relative importance in the design of a practical fibre optic communications network.

In Chapter 2, the bound electromagnetic modes of the weakly guiding dielectric optical fibre of circular cross section with an arbitrary dielectric profile in the core and an infinite uniform cladding are presented in the azimuthal travelling wave form, viz. exp $\{-i \ell \phi\}$ variation. These results are derived within the approximation 
of ignoring all terms in the gradient of the dielectric permittivity. These modal fields are used as the basis of all the analysis of the remaining chapters.

In Chapter 3, the philosophy of the Volume Current Method for the calculation of the radiation loss due to slight imperfections in the ideal straight optical waveguide is presented. In particular, the radiation induced by small weak isolated dielectric irregularities and fluctuation in the core radius are analysed by this technique.

Comparisons are made with the exact treatment (to first order in the perturbation) of Coupled Mode Theory, to display the validity of the method.

In Chapter 4, the breakdown of the Volume Current Method for paraxially directed radiation is discussed and a correction to the Volume Current Method is formulated, so that the simplicity of the analysis via the Volume Current Method is retained but the validity of the results extended. The correction based upon the harmonic time equivalent of the "method of images" in statics is applied to both planar and circular cylindrical structures.

In Chapter 5, the philosophy of the Surface Current Method which is derived from the Stratton-Chu Integral, is presented and applied to determine the tunnelling leaky mode power attenuation coefficient for the tunnelling leaky modes of arbitrary dielectric profile. In this chapter, we demonstrate the relative simplicity of the Stratton-Chu Integral for the radiation fields of the weakly guiding dielectric optical fibre when the azimuthal travelling wave modes are used.

In Chapter 6, this Surface Current Method is applied to the study of radiation losses due to slow bends in dielectric optical waveguides. The modal power attenuation coefficient of a mode incident on a planar bend of constant curvature is derived for slabs and fibres and compared with previously reported results. Ray optical analyses are used to elucidate the physical nature of the approximations utilised in the analysis and from those arguments, restrictions on the radius of curvature for which no significant field deformation and mode coupling 
occurs, are presented.

In Chapter 7, we summarise the major conclusions of this thesis and suggest directions for further research in this field. 
Notes on the text:

(1) References are numbered consecutively and listed at the end of each chapter.

(2) The equations are numbered consecutively within each section. In the notation ( $($ m. $n), Z$ is the chapter number, $m$ the section number and $n$ the equation number. Equations of another section referred to in the same chapter, are referred to by their section and equation number only, viz. (m.n).

(3) The figures are numbered consecutively in each chapter. 


\section{CONTENTS}

PREFACE ii

PUBLICATIONS · iii

ACKNOWLEDGEMENTS $\quad$ v

ABSTRACT vii

CHAPTER 1. INTRODUCTION 1

1.1 The Ideal Dielectric Optical Fibre 2

1.2 A Qualitative Discussion of Imperfections in the
Dielectric Optical Fibre

$\begin{array}{lll}1.3 & \text { The Practical Optical Fibre } & 14\end{array}$

$\begin{array}{ll}\text { References } & 21\end{array}$

CHAPTER 2. THE BOUND MODES OF THE IDEAL STRAIGHT DIELECTRIC OPTICAL FIBRE $\quad 24$

2.1 Preliminary Results and Notation 25

2.2 The Bound Modes of an Ideal Straight Dielectric Fibre 32

2.2.1 The bound modes of an ideal straight dielectric waveguide

2.2.2 The weakly guiding fibre: $\varepsilon_{1} / \varepsilon_{2} \sim 1$

2.3 Discussion

Appendix A: The Modal Fields of an Optical Fibre with Arbitrary Dielectric Difference

Appendix B: On the Effect of $\nabla \varepsilon$ Terms in Inhomogeneous Circular Cross Sectioned Dielectric Cylinders 56

References

CHAPTER 3. RADIATION FROM IRREGULARITIES IN THE STRAIGHT DIELECTRIC WAVEGUIDE: THE VOLUME CURRENT METHOD

3.1 The Volume Current Method

3.2 The Equivalence of the Volume Current Method and Approximate Coupled Mode Theory 
3.3 Applications of the Volume Current Method 79

3.3.1(a) Radiation loss from isolated irregularities 80

3.3.1(b) The efficiency of dipole sources in excitation of the fundamental mode of a step-index fibre

3.3.1(c) Radiation from a random distribution of dielectric irregularities

3.3.2 Radiation loss due to variations in the radius of the core

Appendix A: The Electromagnetic Fields Induced by Electric and Magnetic Currents

Appendix B: Radiation from an Isolated Irregularitiy

Appendix C: Radiation from an Optical Fibre with Axial Sinusoidal Variations of the Core Radius

References

\section{CHAPTER 4. CORRECTIONS TO THE VOLUME CURRENT METHOD}

4.1 Introduction

4.2 The Philosophy of the Volume Current Method and Application to Planar Structures

4.3 Correction to the Volume Current Method for Cylindrical waveguides

4.3.1 The cylindrical electromagnetic wave reflection coefficient

4.3.2 Volume Current Method correction for cylindrical waveguides

\subsection{Discussion}

Appendix A: Radiation Scattered from an Incident Plane Wave Due to a Sinusoidal Perturbation of the Interface between Two Homogeneous Dielectrics

Appendix B: Radiation from the Even TE Modes of a Symmetric Slab Waveguide Due to Diameter Fluctuations

Appendix C: The Cylindrical Electromagnetic wave Reflection Coefficient of a Convergent wave Incident on a Concentric Cylinder of Arbitrary Dielectric Profile

Appendix D: The Corrected Power Attenuation Coefficient of the $\mathrm{HE}_{11}$ Mode on a Step-Index Fibre Due to Sinusoidal Radius Fluctuations 
CHAPTER 5. CURVATURE INDUCED RADIATION: THE SURFACE CURRENT METHOD

5.1 Introduction

5.2 The Surface Current Method

5.3 Radiation from the Ideal Straight Optical Fibre 167

5.4 Conclusions

Appendix A: The Radiation Fields of the Stratton-Chu Integral

Appendix B: The Radiation Fields from the Azimuthal Travelling Wave Modes and their Relation to the Standing Wave Modes

Appendix C: Radiation from the Modes of the Ideal Straight Dielectric Optical Fibre: The Tunnelling Leaky Modes 184

References

CHAPTER 6. RADIATION FROM BENDS IN OPTICAL WAVEGUIDES

6.1 Introduction

6.2 The Philosophy of the Surface Current Method for the Analysis of Bends in Optical Waveguides

6.3 Radiation from the Bent Symmetric Slab Waveguide

6.4 Radiation from the Bent Optical Fibre

6.5 The Ray Optical Determination of the Critical Bending Radius

228

6.6 Conclusions

Appendix A: Radiation from the Bent Symmetric Waveguide

Appendix B: Ray Optical Analysis of the Radiation Loss in a Bent Slab Waveguide

Appendix C: Radiation from a Bend in an Optical Fibre of Circular Cross Section

Appendix D: Equivalence of the Radiated Power from the Azimuthal Travelling Wave and Standing Wave Modes Due to a Planar Bend

References

CHAPTER 7. SUMMARY AND CONCLUSIONS 


\section{CHAPTER 1}

\section{INTRODUCTION}

The remarkable technological achievements in the manufacture and application of cheap, pure, low-loss, glasses which have proliferated in recent years, have opened the way for optical fibres to replace metal waveguides as the basic guidance mechanisms of communication systems. These technological achievements have, in turn, stimulated the theoretical investigations of dielectric waveguides and electromagnetic wave propagation in general.

Because of the very high frequencies of the visible spectrum, the information carrying capacity of the optical fibre is potentially very high. Their small size and light weight, together with the economy of using one of earth's most abundant materials, namely silica, are but a few of the advantages that make the dielectric optical waveguide an attractive alternative to contemporary systems, as well as heralding a new era in electronics with the development of integrated optical circuitry.

The history and development of the theory and technology of optical waveguides, as they are to this time, have been well documented by men eminently more qualified than the author [1-17]. The reader is referred to these tomes for an overview of the field together with rich detail. It is important to note that the full history of the field has yet to be written as evidenced by the ever-expanding list of relevant publications and texts together with the many international conferences on this topic recently held [18-20] and proposed for the near future.

In this thesis, new theoretical techniques are developed and extant techniques extended to provide a basis for the study of radiative losses in dielectric waveguides. Although the applications of these 
techniques are, in general, restricted to an idealised dielectric waveguide, the techniques, themselves, are readily applicable to the practical optical fibre, and wherever possible this extension will be indicated, the emphasis of this thesis is on the fundamental physical processes occurring in different radiation mechanisms.

The real optical fibre, unlike the theorist's vision, possesses inherent structural and material imperfections which cause some of the energy that would propagate without loss in the absence of these imperfections, to be dissipated as it travels along the fibre. To enlighten the reader, to the semantics of this statement, we must first discuss the theorist's vision, i.e. the ideal dielectric optical fibre.

\subsection{THE IDEAL DIELECTRIC OPTICAL FIBRE}

In this section we investigate the ideal dielectric optical waveguide and discuss the principles of propagation along such waveguides. To simplify the description of these principles, we restrict the discussion to the step-index waveguide. The generalisation of the discussion to the graded index waveguide is straightforward, but would not be profitable at this stage as it would only contribute more detail than is necessary to understand the underlying physical principles of optical fibre transmission.

\section{In its most elementary form, ${ }^{\dagger}$ the ideal dielectric optical} fibre consists of a straight, axially uniform, dielectric cylinder of circular cross-section of radius $\rho$, with a real (non-lossy) dielectric permittivity and magnetic permeability which have well defined mathematical forms, $\varepsilon_{1}(r)\left(=\varepsilon_{0} n_{1}^{2}(r)\right)$ and $\mu_{1}(r)$ respectively, depending only on the radial co-ordinate $r$. Here $\varepsilon_{0}$ denotes the dielectric permittivity of free space and $n$ is the refractive index. This cylinder

T The reader is referred to refs. [12] and [16] to appreciate the simplistic nature of this structure amongst the myriad of aesthetically appealing dielectric structures that have been proposed as light guidance structures. However, it appears from both a theoretical [21] and practical viewpoint that this simple model is highly accurate for practical optical fibres. 
is embedded in an infinite homogeneous dielectric medium with a real dielectric permittivity $\varepsilon_{2}\left(=\varepsilon_{0} n_{2}^{2}\right)$ and magnetic permeability $\mu_{2}$, as in fig. 1. These two regions of the dielectric optical fibre are known as the core and cladding respectively. A necessary condition for the guidance of energy in the core of the fibre is

$$
\varepsilon_{1}^{2}(r)>\varepsilon_{2} \quad \text { for } r<\rho
$$

Intuitively, propagation in ideal dielectric structures is most easily described in geometric optics terminology. In a planar (i.e. slab) homogeneous dielectric core waveguide, as in fig. 2, using the classical Snell's Law for rays incident on the core-cladding interface from the core medium, a ray will suffer no loss in energy upon reflection from the interface if the angle that the ray makes to the normal to the interface, $\alpha$, is greater than the critical angle, $\alpha_{c}$, of the interface. This condition may be written as

$$
\alpha \geqslant \alpha_{c}=\sin ^{-1}\left(n_{2} / n_{1}\right)
$$

Such rays continue to travel unattenuated in the axial direction of the waveguide by total internal reflection from the core-cladding interface.

In the ideal straight dielectric optical fibre with a core of homogeneous dielectric, as in fig. 3, it has been shown that a similar result applies [23] except that the result is stated in terms of the complement of the critical angle, $\theta_{C}\left(=\pi / 2-\alpha_{C}\right)$ and from a geometric optics viewpoint can be related to the angle that the ray makes with the z-direction, $\theta_{z^{*}}$. Rays that are incident at the core-cladding interface at angles $\theta_{\mathbf{z}}$ to the axis that satisfy

$$
\theta_{z} \leqslant \theta_{c}=\sin ^{-1}\left(1-n_{2}^{2} / n_{1}^{2}\right)
$$

suffer no loss in energy upon reflection from the core-cladding interface. As before, these rays can then propagate along the fibre with no attenuation of their energy. The more general class of inhomogeneous

\footnotetext{
+ This geometric optics argument obviously ignores the phase interference phenomena of wave theory, that imposes a consistency condition [22] (or eigenvalue equation) upon the allowed directions of propagating rays.
} 


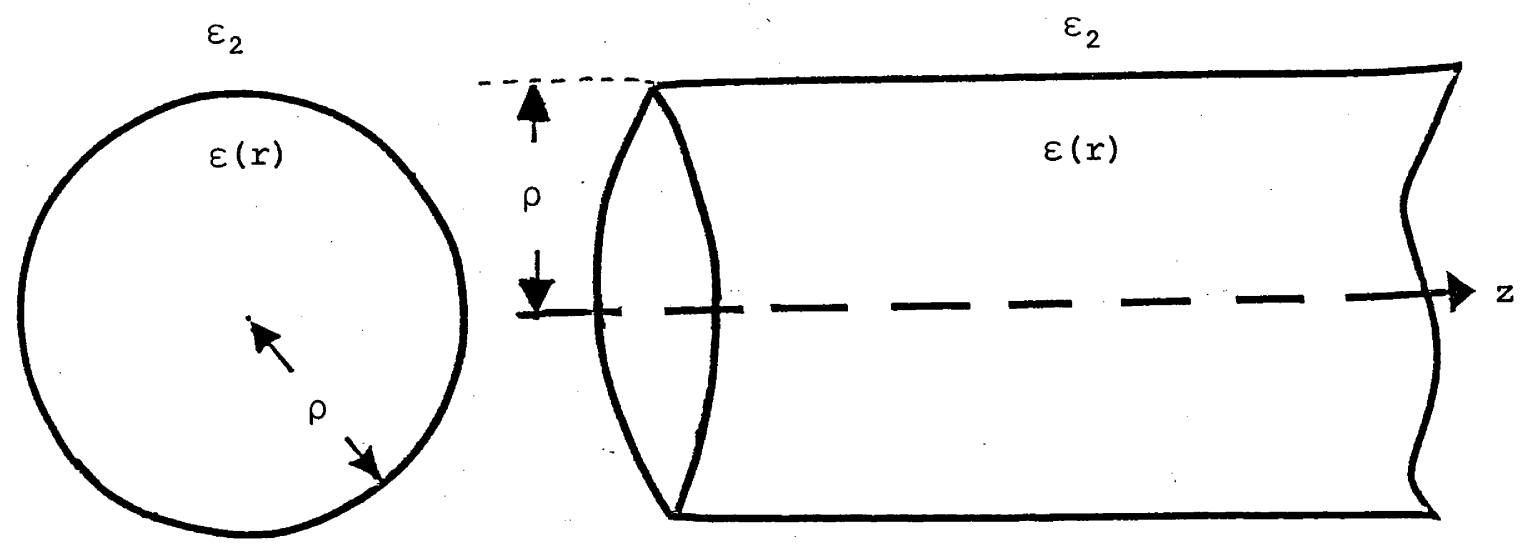

Fig. 1: The ideal dielectric optical fibre of circular cross section. 


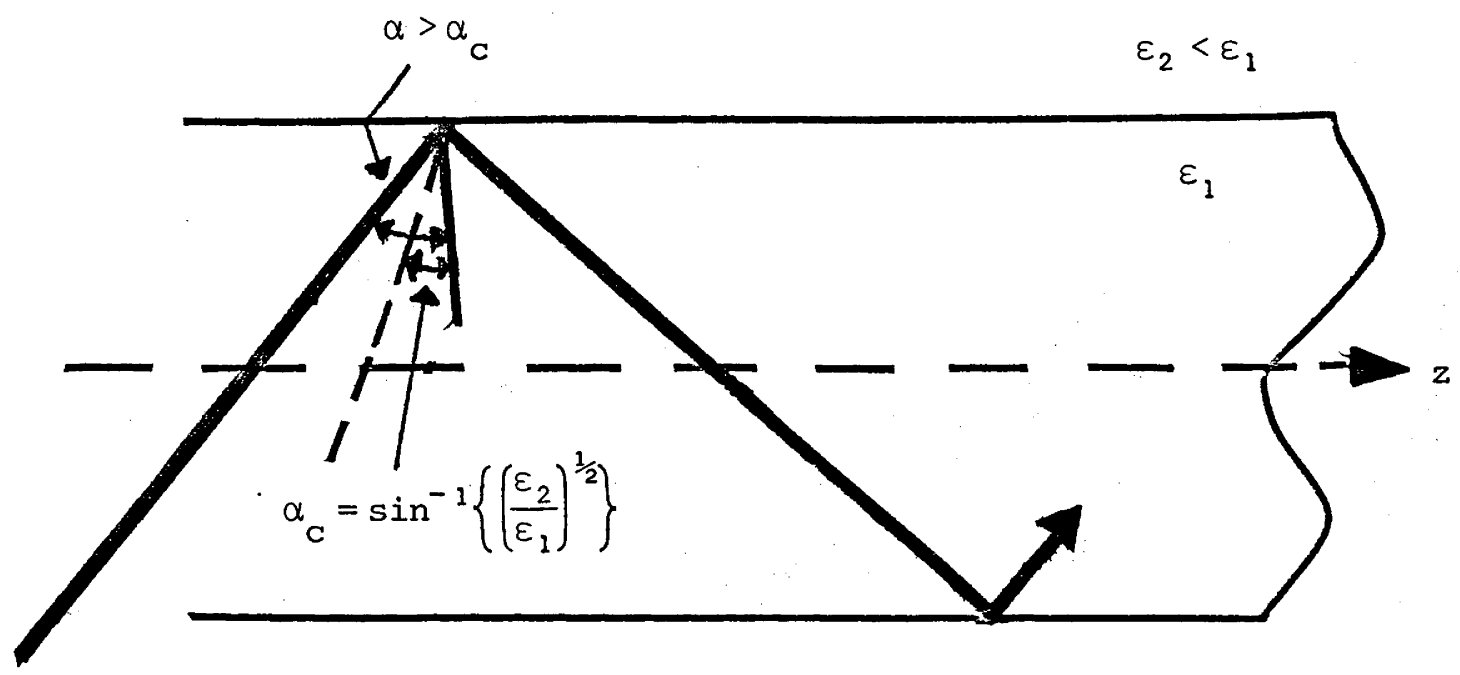

Fig. 2: Total internal reflection of a ray in a slab waveguide. 


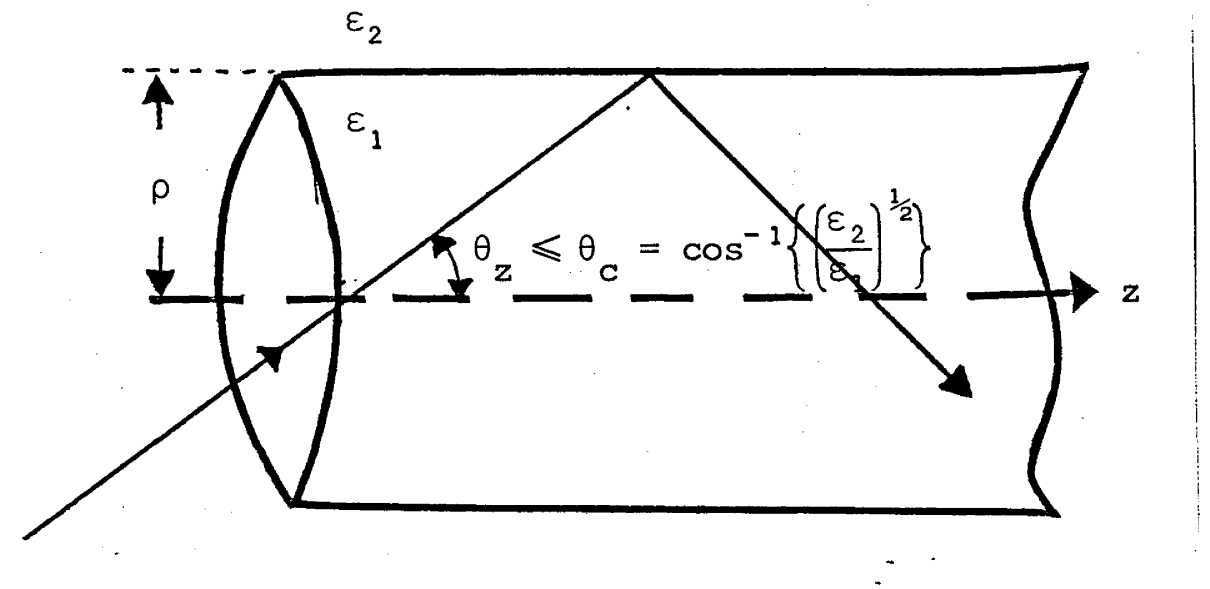

Fig. 3: Rays in circular cylinder dielectric waveguides suffer total internal reflection from the core-cladding interface if $\theta_{z} \leqslant \theta_{C}=\cos ^{-1}\left[\left(\varepsilon_{2} / \varepsilon_{1}\right)^{\frac{1}{2}}\right]$. 
dielectric core waveguides have been shown to exhibit similar characteristics $[13,24]$. This criteria for trapped rays (equation (2)) is strictly a consequence of the wave nature of the light. The electromagnetic counterpart of rays, i.e. modes, must propagate in the $z-$ direction at a speed less than the speed of light in the bulk material with the core dielectric permittivity so that travelling waves can exist in the core. A trapped mode propagates along the fibre with a speed that is greater than the speed of light in the cladding, thus ensuring that no bound mode energy can radiate away from the core. The rays that are excluded by equation (2), that by classical geometric optics should be trapped, represent the tunnelling leaky rays [10] which are only very slowly attenuated in comparison to the refracting rays.

Even in the elementary form of the optical fibre described above, there can be many variations on the basic theme. However, there are three basic types of dielectric optical fibre, as in figs. 4a-c, which are generally accepted as the most practicable for optical fibre systems. The essential distinction between them is the size of the core compared to the wavelength of the light.

The structures of figs. $4 a, b$ are known as step-index and graded-index multimode dielectric optical fibres. Such waveguides are generally characterised by core dimensions many times greater than the wavelength of the light. To be more explicit, Snyder [25] has shown that for weakly guiding step-index waveguides, a parameter $\mathrm{V}$ completely specifies the behaviour of the electromagnetic modes of the waveguide for a monochromatic field, where $\mathrm{V}$ is defined by

$$
v=\frac{2 \pi \rho}{\lambda}\left(n_{1} \sin \theta_{c}\right)
$$

or

$$
\mathrm{V}=\mathrm{k}_{1} \rho \sin \theta_{c^{\prime}},
$$

where $k_{1}=2 \pi n_{1} / \lambda$ is the wavenumber in the core for light with wavelength $\lambda$ in free space. The light acceptance capabilities of the fibre excited by a monochromatic source and the characteristics of the individual modes are completely determined by $\mathrm{V}$ and the acceptance angle $\theta_{\mathrm{C}}$.

A multimode waveguide is characterised by the parameter $\mathrm{V} \gg>1$ 


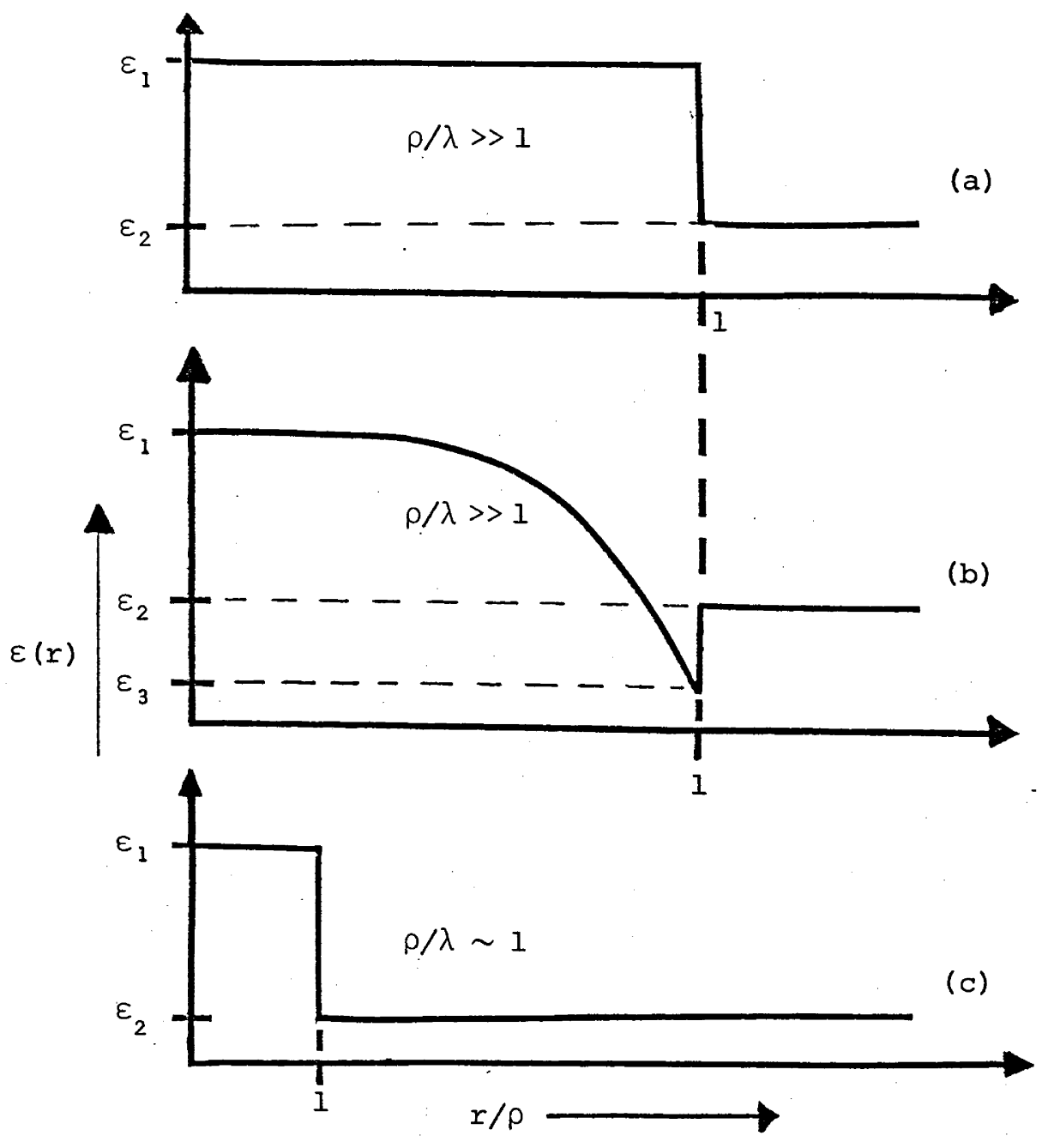

Fig. 4: The dielectric profiles of optical waveguides:

(a) The multimode step-index waveguide.

(b) The multimode graded-index waveguide.

(c) The monomode step-index waveguide. 
and as the name suggests is capable of supporting many bound (nonattenuating) electromagnetic modes. For a monochromatic source each of these modes carries energy along the fibre at different speeds, corresponding to the modal group velocities of the respective modes [26] As the frequency of the source is changed, the normalised frequency $V$ changes according to equation (3), hence the modal eigenvalue varies. Associated with this variation in the frequency there is a corresponding change in the modal group velocity. Thus when a temporal pulse of light enters the fibre, the energy then travels along the waveguide distributed amongst the modes each travelling at a different speed along the axis. This causes the pulse to spread as it travels down the fibre. This effect is known as the modal dispersion of the waveguide, and proves to be the main disadvantage of multimode waveguides. Because of the non-uniform dielectric profile of the graded-index fibre, the effect of modal dispersion can be much reduced [27] in comparison with the step-index fibre, with the same maximum dielectric difference. To reduce this modal dispersion, practical optical fibres have small differences between the core and cladding dielectric permittivities, i.e.

$$
\frac{\Delta \varepsilon}{\varepsilon_{1}}=\sin ^{2} \theta_{c}=\frac{\left(\varepsilon_{1}-\varepsilon_{2}\right)}{\varepsilon_{1}} \ll 1
$$

In fig. 4c the most attractive structure for an optical fibre system is shown. Its cross-sectional dimensions are such as to allow only one bound mode to exist. This structure is appropriately known as the monomode step-index fibre and because of this, the modal dispersion present in monomode fibres is due completely to the material dispersion of the core and cladding dielectrics. The main disadvantage of the monomode fibre is its small core size, making handling the fibre and aligning joins in fibres very difficult. The main attraction of these waveguides is the very small impulse response allowing them to carry much more information than multimode waveguides.

The inter-relationship between the geometric optics and electromagnetic descriptions of propagation in optical waveguides alluded to previously, requires discussion. For the step-index waveguide, the asymptotic evaluation $[2,28]$ of the modal electromagnetic fields, for fibres with $V \gg 1$, shows that these fields are formed by a 
family of rays, with each ray of this family incident on the corecladding boundary at the same angle to the normal. Using this procedure, we can define the characteristic angles $\left(\theta_{z}, \gamma\right.$ ) as in fig. 5. (relative to the core-cladding interface) of the rays that constitute the geometric optics equivalent of an electromagnetic mode. For multimode gradedindex fibres similar results apply [29], although the rays cannot be referred to a common interface as in the step-index fibre. (This lack of a common interface for all rays in the graded-index fibre also restricts the use of the equivalent characteristic angles as used in the step-index waveguide.)

The classical electromagnetic theory approach to the study of propagation in dielectric waveguides has been shown to be overly complicated for multimode step-index waveguides [10] and proves to be mathematically intractable for all but a special class of graded-index waveguides. A classical physics approach using geometric optics has enjoyed great success for the multimode waveguides, describing the fundamental mechanisms of the fibre optic transmission with elegant simplicity. For monomode and small $V$ fibres $(V<20$ for the step-index waveguide), electromagnetic theory must be used as the wave nature of the light becomes significant.

The excessively mathematical analysis of dielectric optical fibres using electromagnetic theory obscures insight into the occurring physical processes. It is often observed in articles in this field that the authors revert to geometric optics wherever possible for explanation and insight. Indeed, as in quantum mechanics, wave theory is often cross-fertilised to good effect by its classical counterpart, the particulate theory. It is therefore the wish of the author to refrain from either dogma and wherever possible to relate the modal analysis to the geometric optics with the hope that this marriage bears the fruit of greater understanding.

Now that we have defined the ideal straight dielectric optical fibre, we are in a position to discuss in a qualitative manner the effect of structural imperfections on propagation in dielectric optical fibres. 

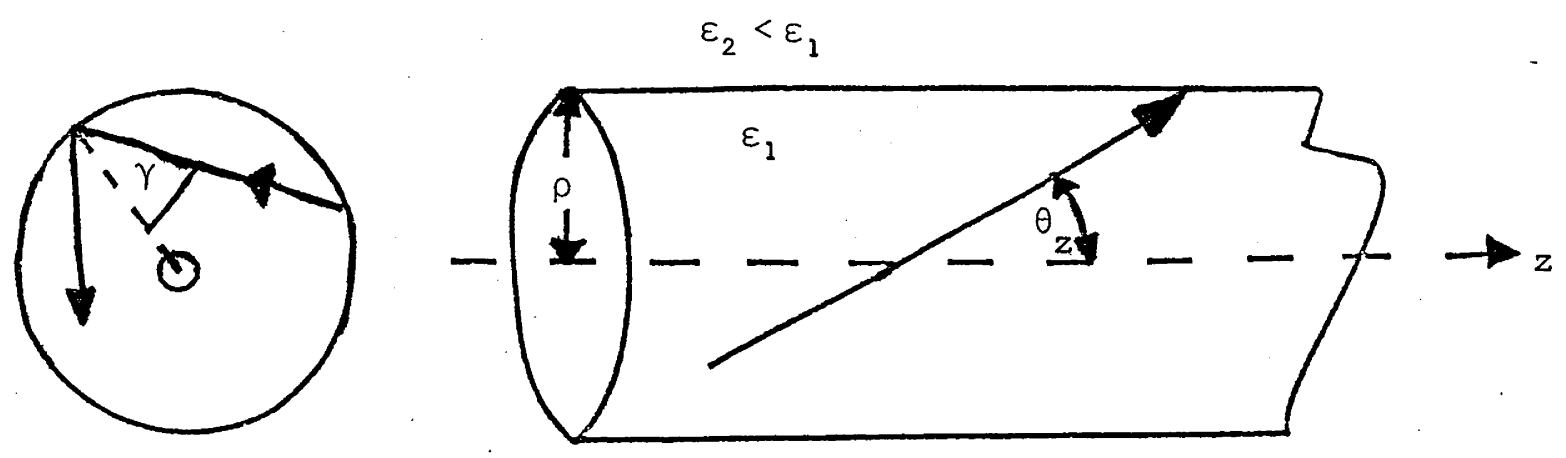

Fig. 5: The characteristic ray directions in the step-index optical fibre $\theta_{z}$ is the angle to the $z$ direction and $\gamma$ is the angle between the radial direction and the ray direction in the cross section of the fibre at the core-cladding interface. 


\subsection{A QUALITATIVE DISCUSSION OF IMPERFECTIONS IN THE DIELECTRIC OPTICAL FIBRE}

The theoretical study of propagation of electromagnetic waves in ideal straight dielectric optical waveguides remains an active area of research with contemporary emphasis on propagation in inhomogeneous dielectric cored waveguides. Along with such fundamental work, an examination of the effect of irregularities and imperfections in the dielectric structure, induced in the manufacture and application of such waveguides must follow since perfection appears to be beyond the capabilities of man (or at least technologists). For want of economy of manufacture and facility of utilisation, the real fibre possesses deviations from the ideal guiding structure such as the inherent surface roughness of the core-cladding interface in a step-index fibre, and bending of the fibre axis. The effect of irregularities is to scatter the incident power, thereby altering the modal power density profile as it travels down the fibre. Studies are necessary to determine the allowable tolerances on the type, size and density of such imperfections in order that the dielectric optical waveguide retains its attractive features.

The appeal of the dielectric optical fibre is in its large information carrying capacity; therefore the most important consideration in dielectric optical waveguides is the evolution of a temporal pulse of light as it travels down the optical fibre as this will determine the ultimate information carrying capacity of the fibre. In general, the imperfections manifest themselves in two significant ways in pulses, namely:

(a) A distortion of the input pulse, by a redistribution of a portion of the incident guided energy among the bound modes of the waveguide, thereby producing a change in the bandwidth, or impulse response, of the fibre; and

(b) A reduction in the total energy carried in the pulse due to a portion of the incident energy being scattered into the radiation modes which are rapidly attenuated. This radiation loss can also affect the impulse response of the multimode fibre since for most radiative mechanisms the magnitude of the 
loss is dependent on the mode initially excited.

Of these two manifestations of irregularities, only the radiative losses are extant in monomode fibres.

To incorporate the effect of irregularities into a study of the evolution of pulses in dielectric optical fibres, it is therefore necessary to study the effects of individual scattering mechanisms on isolated modes of the structure.

The bulk of this thesis is concerned with the development of simple techniques to determine the radiative losses due to irregularities and structural imperfections. The conventional coupled Mode Theory treatment [9], although an excellent tool for the study of the redistribution of power among the bound modes, can only be used with much difficulty for the study of the radiative losses, owing to the complex nature of the radiation modes. With the techniques presented here, it is possible to circumvent these tedious Coupled Mode Theory analyses.

There are two physically distinct classes of radiative loss mechanisms in dielectric optical fibres, namely:

(1) Radiation losses from straight fibres with material and structural imperfections. Some typical examples are the inherent roughness of the core-cladding surface and fluctuations in the dielectric permittivity induced in the manufacturing process and material impurity.

(2) Curvature induced radiation arising from the inherent curvature of the core-cladding interface and due to deformations of the fibre such as bends in the fibre axis induced in laying a practical fibre system.

From experience, it has been found that these two distinct classes of radiative loss mechanism require two different techniques to treat them. In the practical weakly guiding optical fibre obeying equation (1.4) the type (1) mechanisms can be studied by conventional perturbation analysis of the ideal straight dielectric optical fibre $[3,9,10]$, where the perturbations relative to the straight fibre are 
everywhere small, as in fig. 6. However, such a perturbation analysis is inapplicable for the analysis of curvature induced radiation losses since, as in the case of a bend in the fibre axis, the perturbations about the unperturbed straight fibre can be very large as demonstrated in fig. 7. A perturbation analysis can be used for bound mode coupling in bends by the local normal mode $[9,30]$ formulation of coupled mode theory, since the bound modes are strongly localised near the core of the fibre, but is inapplicable for radiative losses due to the nonlocalised nature of the radiation modes.

In this thesis, two conceptually related techniques known as the Volume Current Method and the Surface Current Method are developed to treat the two classes of radiative loss mechanisms. The theoretical analysis of the effect of irregularities is by no means a new field. Previous studies, however, have been restricted to metal clad waveguides where radiative loss is of little significance. The weakly guiding fibre has the advantage that, in most cases where applicable, first order perturbation theory is very accurate, since most irregularities are structural and thus the magnitude of the dielectric perturbation is $\Delta \varepsilon$, which is very small.

\subsection{THE PRACTICAL OPTICAL FIBRE}

The discussion of the dielectric optical waveguide in the previous sections has been confined to a somewhat esoteric structure, i.e. a waveguide with a cladding of infinite extent. For fiscal, as well as spatial, economy the practical waveguide consists of a cladding of finite extent as in fig. 8. Outside this cladding, there is usually a thin highly lossy coating to effectively isolate the electromagnetic modes of the individual fibre and thus prevent any cross-talk [30] between neighbouring fibres. The thickness of the inner cladding is usually sufficient to confine the fields of the majority of the bound modes of the ideal waveguide.

This thesis is primarily concerned with the study of the effect of material and structural irregularities in the ideal dielectric 


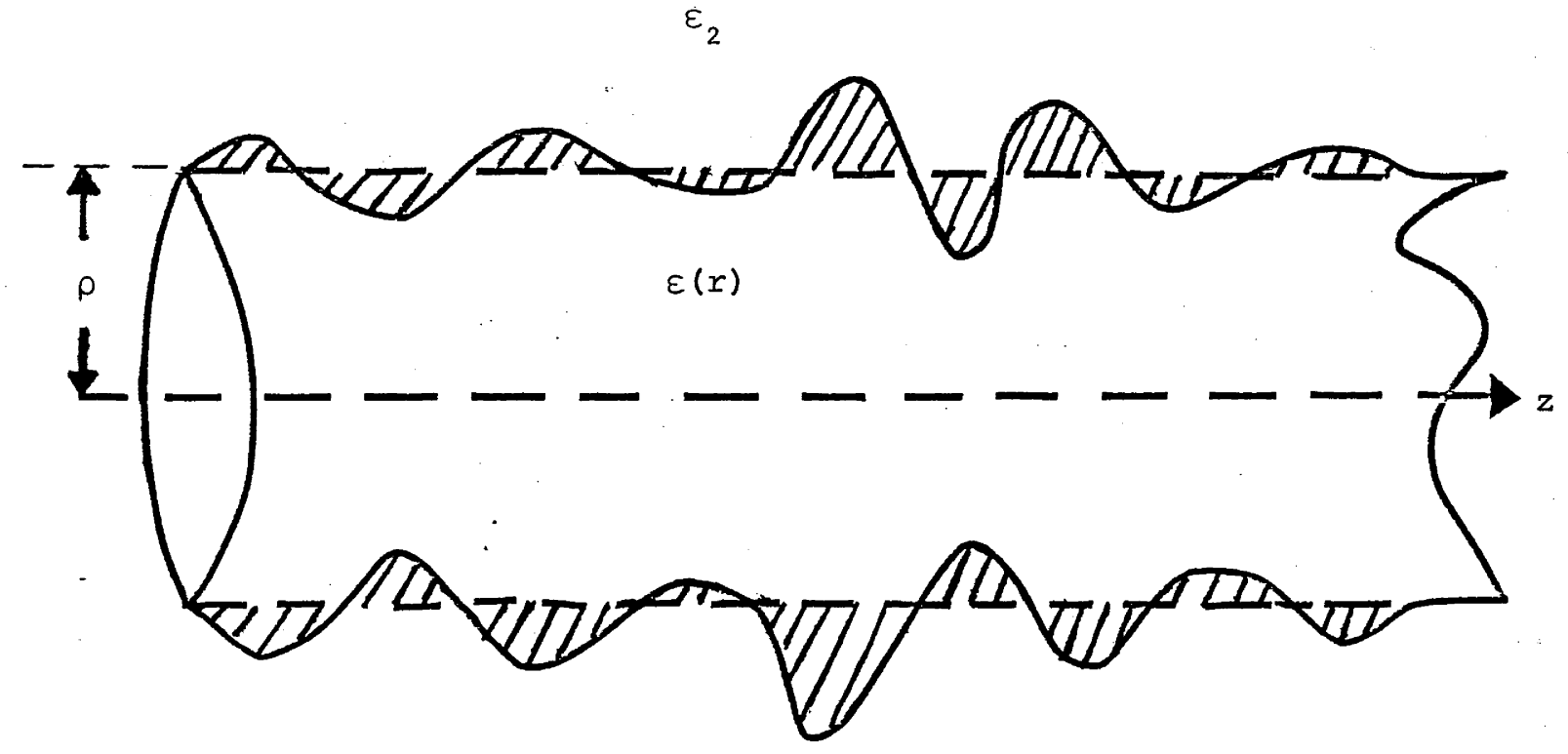

Fig. 6: Dielectric optical waveguide with slight imperfections. The shaded areas represent the perturbations to the ideal dielectric optical fibre whose unperturbed radius is indicated by the dotted lines. 


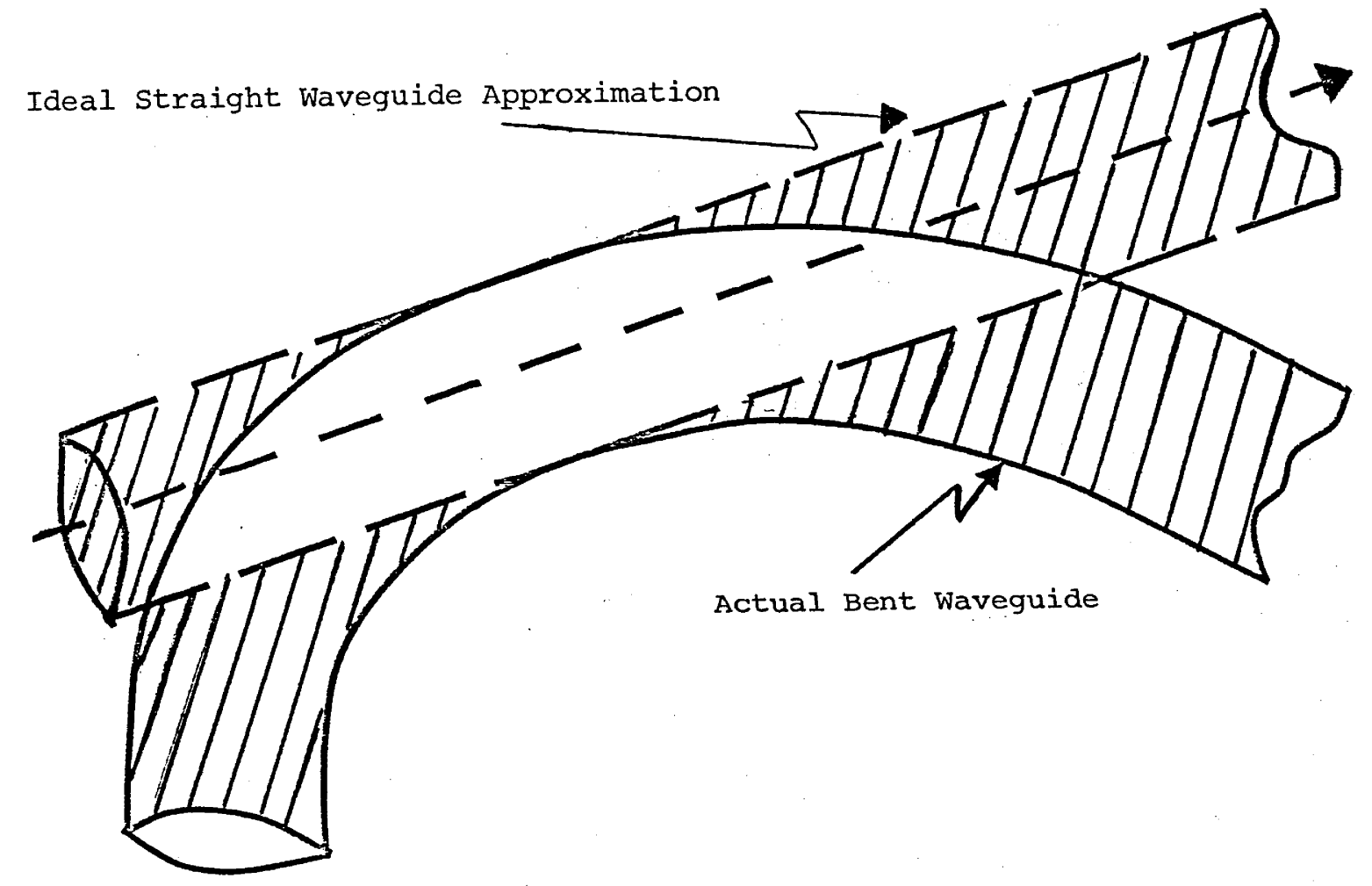

Fig. 7: The shaded regions indicate the perturbation to the ideal waveguide. The perturbation of the bent waveguide is no longer slight with respect to the ideal straight guide. 


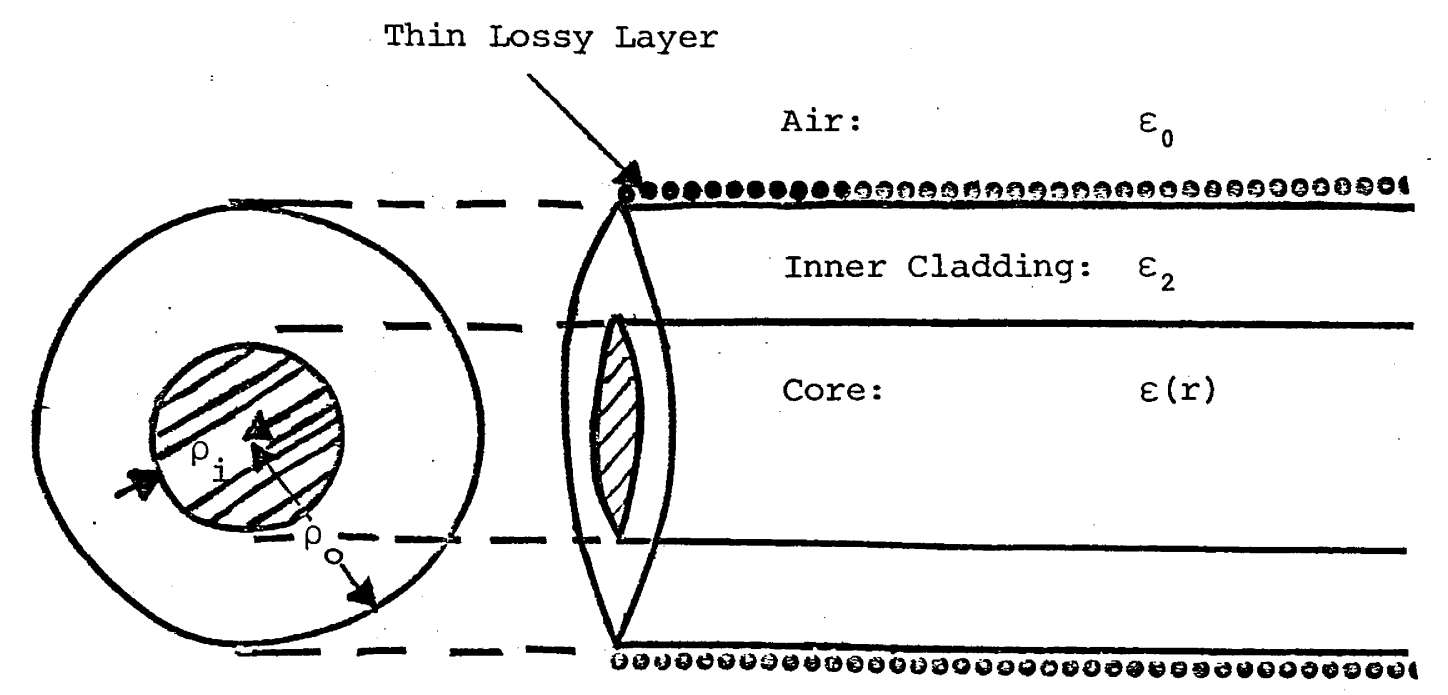

Fig. 8: The practical optical fibre. Such a structure usually possesses a highly lossy thin outer layer in order to reduce crosstalk between optical waveguides. This is shown in the diagram as the dots. 
waveguide of section 1.1. To determine the significance for the practical fibre of the results and theories presented here, we must first discuss the effect of the finite extent of the cladding. For radiative losses this finiteness is significant in two respects, viz.,

(1) The effect of the finite cladding on the functional forms of the bound modes; and

(2) The effect of the presence of another interface beyond the core-cladding boundary on the radiation fields generated by the imperfections.

If the finite extent of the cladding significantly alters the intensity of a mode at the location of an irregularity relative to the intensity of the corresponding mode of the ideal waveguide, then the magnitude of the power scattered from the mode by an irregularity in the waveguide will be affected.

The assumption of an infinite cladding has been shown by Clarricoats and Chan [21] not to be particularly critical in determining the behaviour of the bound modes except very close to cutoff, where the fields extend far into the cladding and thus strongly experience the presence of the extra boundary. It may be expected to be of more importance in the case of leaky modes whose fields extend further from the core. In fact, if the medium outside a cladding of finite extent were of refractive index $n_{3}<n_{2}$, then some of the leaky modes will become proper modes of the cladding structure (the so-called "cladding modes"). On the other hand, if $n_{3}>n_{2}$ all modes are leaky [12] due to frustrated total internal reflection [2]. Thus, for the majority of the modes of the practical fibre, the finite extent of the cladding does not affect the amount of power scattered from an irregularity. However, the existence of this outer boundary will strongly determine how much of this scattered power is in the form of radiation from the waveguide.

The important consideration of the effect of this additional interface on the radiation fields induced by any imperfections in the waveguide must be discussed relative to the radiation loss mechanism generating the fields. If the radiation emanates from the region consisting of the core and inner cladding, the effect of the outer 
interface between the cladding and the medium of dielectric $n_{3}$, depends on the relative magnitudes of $n_{2}$ and $n_{3}$. For $n_{2}<n_{3}$, the radiation will be refracted by the interface, with transmission coefficient depending on the generalised Fresnel's Laws. For $n_{3}<n_{2}$, the radiative power with $\theta_{z} \leqslant \sin ^{-1}\left(1-n_{3}^{2} / n_{2}^{2}\right)^{\frac{1}{2}}$ will be totally internally reflected and constitute energy coupled into the cladding modes. The remainder will be refracted with the transmission coefficient determined by the generalised Fresnel's Law for the interface between $n_{2}$ and $n_{3}$.

For the situation in which the cladding absorption loss is high, as in the polymer clad fibres [31], the radiation emanating from the core may be completely absorbed by the cladding and thus never experience the outer cladding surface and the approximation of the infinite cladding should prove to be excellent for determination of the magnitude of the loss. In this case, the lossy cladding acts as a sink that absorbs the radiative energy -

If the radiation emanates from beyond this outer interface, such as is the case for slowly leaking tunnelling leaky modes, the magnitude of loss depends on the intensity of the fields at this outer surface [32]. Such a situation cannot be treated by the modes of the two region fibre and one must resort to analysis of three or more, region fibres. For the modes of the fibre that are tightly bound to the core, the fields at the outer cladding interface should be well approximated by the amplitude of the modal field at this position for the fibre with an infinite cladding, without resorting to the more complicated analysis of the multi-region waveguide.

These effects for the radiation emanating from beyond the outer cladding interface will not be presented in this thesis except where the simplifying assumption of tightly bound modes is used. Some studies by Kawakami et aZ. [33] have been done on W-type slabs to determine these effects. More detailed studies of the effect of the finite cladding on curvature induced losses are required.

To study the effects of the radiative loss mechanisms of the modes below cutoff in the fibre with finite cladding, i.e. the tunnelling leaky modes [10], the cladding modes of Clarricoats and Chan 
[21] should be used. However, as this thesis attempts to develop a set of simple techniques for the study of radiative loss mechanisms, we shall not complicate the study with these considerations. The effect of the radiation mechanisms on these cladding modes will be left for future study. 


\section{REFERENCES}

[1] N.S. Kapany: Fibre Optics, Principles and Applications (Academic Press, New York, 1967).

[2] N.S. Kapany and J.J. Burke: Optical Waveguides (Academic Press, New York., 1972).

[3] D. Marcuse: Light Transmission Optics (Van Nostrand Reinhold, New York, 1972).

[4] R.D. Maurer: "Glass fibres for optical communications", Proc. I.E.E.E. 61, 452 (1973).

[5] D. Marcuse: "Optical fibres for communications", Radio Elec. Eng. 43 , 655 (1973).

[6] S.E. Miller, E.A.J. Marcatili and Tingye Li: "Research toward optical-fibre transmission systems", Proc. I.E.E.E. 61, 1703 (1973).

[7] D. Gloge: "Optical fibres for communication", App 2. Optics 13, 249 (1974).

[8] H.F. Taylor and A. Yariv: "Guided wave optics", Proc. I.E.E.E. 62, 1044 (1974).

[9] D. Marcuse: Theory of Dielectric Optical Waveguides (Academic Press, New York, 1974).

[10] A.W. Snyder: "Leaky-ray theory of optical waveguides of circular cross-section", Appl. Phys. 4, 273 (1974).

[11] C.P. Sandbank: "Fibre optics communications: a survey", Elec. Commun. 50, 20 (1975).

[12] P.J.B. Clarricoats: "Theory of optical fibre waveguides: a review" in Progress in Optics, vol. 14 (E. Wolf, ed.) (NorthHolland, Amsterdam, 1976).

[13] D. Gloge: "Propagation effects in optical fibers", I.E.E.E. Trans. MTT-23, 106 (1975).

[14] W.B. Allan: Fibre Optics, Theory and Practice (Plenum Press, New York, 1973).

[15] M.K. Barnoski: Fundomentals of Optical Fiber Communications (Academic Press, New York, 1976). 
[16] J.A. Arnaud: Beam and Fiber Optics (Academic Press, New York, 1976).

[17] J.E. Midwinter: "Trends in optical fibre transmission research", Nature 261, 371 (1976).

[18] O.S.A./I.E.E.E. Meeting on Optical Fibre Transmission, Williamsburg, 1975.

[19] European Conference on Optical Fibre Communication, London, September 1975. (IEE Conference Publication 132.)

[20] 2nd European Conference on Optical Fibre Communication, Paris, September 1976.

[21] P.J.B. Clarricoats and K.B. Chan: "Propagation behaviour of cylindrical dielectric-rod waveguides", Proc. I.E.E. 120, 1371 (1973).

[22] K.G. Budden: Radio Waves in the Ionosphere: the Mathematical Theory of the Reflection of Radio Waves from Stratified Ionised Layers (Cambridge Univ. Press, 1961).

[23] D. Marcuse: "Cutoff condition of optical fibres", J. Opt. Soc. Am. 63, 1369 (1973).

[24] M.J. Adams, D.N. Payne and F.M.E. Sladen: "Leaky rays on optical fibres of arbitrary (circularly symmetric) index profiles", Electron. Letts. 11, 238 (1975).

[25] A.W. Snyder: "Asymptotic expressions for the eigenfunctions and eigenvalues of a dielectric or optical waveguide", I.E.E.E. Trans. MTT-17, 1130 (1969).

[26] J.D. Jackson: Classical EZectrodynamics (Wiley, New York, 1962).

[27] D. Marcuse: "The impulse response of an optical fiber with parabolic index profile", BelZ Syst. Tech. J. 52, 1169 (1973).

[28] A.W. Snyder and D.J. Mitchell: "Leaky rays on circular optical fibres", J. Opt. Soc. Am. 64, 599 (1974).

[29] A. Ankiewicz and C. Pask: "Geometric optics approach to light acceptance and propagation in graded index fibres", Optical and Quantum Electronics $\underline{9}, 87$ (1977).

[30] A.W. Snyder: "Mode propagation in optical waveguides", Electron. Letts. 6, September (1970).

[31] S. Tanaka, K. Inada, T. Akimoto and M. Kozima: "Silicon-clad fused-silica-core fibre", Electron. Letts. 11, 153 (1975).

[32] J.A. Stratton: Electromagnetic Theory, pp.464-468 (McGraw-Hill, New York, 1941). 
[33] S. Kawakami, M. Miyagi and S. Nishida: "Bending losses of dielectric slab optical waveguide with double or multiple claddings: Theory", Appl. Optics 14, 2588 (1975). 
CHAPTER 2

\section{THE BOUND MODES OF THE IDEAL STRAIGHT DIELECTRIC OPTICAL FIBRE}

In the proceeding chapters, the bound modes of the ideal straight dielectric optical fibre of circular cross-section will be used as the basis for analysis of radiative loss mechanisms in the dielectric waveguide. The present state of the art of dielectric waveguides requires techniques that are useful for both homogeneous (step-index) and inhomogeneous dielectric cored waveguides. To this end, this chapter presents a general set of of bound modes for the simplistic (two region) form of the dielectric optical fibre, with an arbitrary radial variation of the dielectric profile $\varepsilon(r)$ in the core. With this set of modes the analysis of radiative loss mechanisms will be studied and only for quantitative discussions will a definite form for the profile be considered. For these quantitative results the modes of the step-index fibre will be used.

Studies of the propagation behaviour of a cylindrical dielectric embedded in an infinite medium of different dielectric permittivity began with a theoretical treatment by Hondros and Debye [1] at the turn of the century. These were followed much later by theoretical and experimental investigations relevant to the propagation of microwave signals along dielectric rod waveguides and to the preliminary analysis of circular waveguides containing ferrite rods [2-6]. The possible use of the structure as an optical waveguide is attributed to Snitzer [7] whose investigations stimulated interest in optical fibre waveguides for telecommunication applications.

Due to the complexity of the exact solutions of Maxwell's equations for cylindrical structures, analysis using these modes proves 
cumbersome. The computational analysis of the modes far from cutoff, in Snitzer's theoretical study, first suggested the existence of a simplified set of modes for these dielectric structures but it was Snyder [8] in 1969 who first derived such a simplified modal electromagnetic field for step-index waveguides based on the assumption of a weakly guiding dielectric optical fibre in which the dielectric difference between the core and cladding dielectrics is small. This approximate set of modes facilitate calculations of all modal characteristics. These modal forms together with the simplified eigenvalue equation have been shown to be excellent approximations for the fibre with infinite cladding for all frequencies [9]. This "weak guidance" approximation can be extended to include a more general class of dielectric profiles and in section 2.2 such a set of approximate modes are presented. In the final section of this chapter a discussion of the results obtained in the previous sections is given along with the modal fields for the step-index fibre, which will be used to discuss quantitatively the radiative loss mechanisms to be studied in the following chapters.

We begin with a brief resumé of some classical electromagnetic theory with specific reference to cylindrical structures of circular cross-section.

\subsection{PRELIMINARY RESULTS AND NOTATION}

The fundamental quantities involved in electromagnetic phenomena are the electric field intensity $\underset{\sim}{E}$, the electric displacement $\underset{\sim}{D}$, the magnetic field intensity $\underset{\sim}{H}$ and the magnetic flux density $\underset{\sim}{\sim}$. The equations governing their behaviour in a linear, source-free medium of dielectric permittivity $\varepsilon$ and magnetic permeability $\mu$ are Maxwell's equations [10]

$$
\begin{aligned}
& \underset{\sim}{\nabla} \times \underset{\sim}{E}=-\frac{\partial \underline{\sim}}{\partial t}, \\
& \underset{\sim}{\nabla} \times \underset{\sim}{H}=\frac{\partial \underset{\sim}{D}}{\partial t}, \\
& \underset{\sim}{\nabla} \underset{\sim}{D}=0,
\end{aligned}
$$




$$
\underset{\sim}{\nabla} \cdot \underset{\sim}{B}=0
$$

and the constitutive relations

$$
\begin{aligned}
& \underset{\sim}{D}=\underset{\sim}{E}, \\
& \underset{\sim}{B}=\mu \underset{\sim}{H},
\end{aligned}
$$

where $\varepsilon$ and $\mu$ are tensors in an anisotropic medium.

At a dielectric interface (a discontinuity in the dielectric permittivity) the electromagnetic fields must also satisfy the boundary conditions

(i) components of $\underset{\sim}{E}$ and $\underset{\sim}{H}$ tangential to the interface are continuous across the interface, and

(ii) components of $\underset{\sim}{D}$ and $\underset{\sim}{B}$ normal to the interface are continuous.

In the absence of static electric charges or magnetic fields, equations (3) - (4) and boundary conditions (ii) are a direct consequence of equations (1) - (2) so that, in the time-varying situations with which we are concerned, only the latter equations and boundary conditions (i) need be considered [11].

Any well-behaved complex field $\underset{\sim}{F}(\underset{\sim}{\underline{x}}, t)$ (electric or magnetic) which satisfies these equations and conditions can be Fouriertransformed in time to give

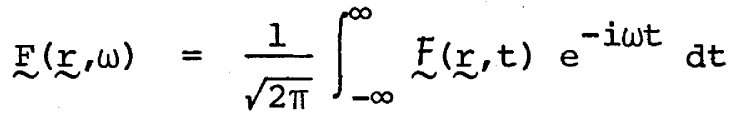

$$
\begin{aligned}
& \left.\underset{\sim}{F}(\underset{\sim}{r}, t)=\frac{1}{\sqrt{2 \pi}} \int_{-\infty}^{\infty} \underset{\sim}{\mathbb{F}} \underset{\sim}{\underline{r}}, \omega\right) e^{i \omega t} \mathrm{~d} \omega
\end{aligned}
$$

so that it is only necessary to consider time-harmonic fields of the form $\underset{\sim}{\mathbb{F}}(\underset{\sim}{\sim}, \omega) e^{i \omega t}$, where $\omega$ is the angular frequency of the field. Throughout this thesis, the fields to be studied will be complex, timeharmonic fields, it being understood that the physical field is given by the real part of this quantity. 
For these harmonic fields, equations (1) - (2) can be rewritten using equations (5) - (6) to give

$$
\underset{\sim}{\nabla} \times \underset{\sim}{\mathrm{E}}=-i \omega \mu \underset{\sim}{\mathrm{H}}
$$

and

$$
\underset{\sim}{\nabla} \times \underset{\sim}{\mathbb{H}}=i \omega \varepsilon \underset{\sim}{\sim},
$$

where $\underset{\sim}{\mathrm{E}}$ and $\underset{\sim}{\mathrm{H}}$ are the Fourier transforms (in time) of $\underset{\sim}{\mathbb{E}}$ and $\underset{\sim}{H}$.

Taking the curl of equation (9), substituting equation (10) and using the identity (in a cartesian co-ordinate system),

$$
\underset{\sim}{\nabla} \times(\underset{\sim}{\nabla} \times \underset{\sim}{E})=\underset{\sim}{\nabla}(\underset{\sim}{\nabla} \underset{\sim}{E})-\underset{\sim}{\nabla^{2}} \underset{\sim}{E}
$$

we find

$$
{\underset{\sim}{\nabla}}^{2} \underset{\sim}{E}+\underset{\sim}{\nabla}[\underset{\sim}{E} \cdot \underset{\sim}{\nabla}(\ln \varepsilon)]+\underset{\sim}{\nabla}(\ln \mu) \times(\underset{\sim}{\nabla} \times \underset{\sim}{E})+\omega^{2} \mu \varepsilon \underset{\sim}{\mathrm{E}}=0
$$

and similarly the equation for $\underset{\sim}{\mathrm{H}}$ is obtained by replacing $\underset{\sim}{\mathrm{E}}$ by $\underset{\sim}{\mathrm{H}}$ and interchanging $\varepsilon$ and $\mu$. The ( ) indicates that the $\underset{\sim}{ }$ operator acts on the term in the brackets only.

In graded-index fibres, where $\varepsilon, \mu$ are not homogeneous, we can consider the inhomogeneity of the dielectric permittivity and magnetic permeability as perturbations in homogeneous waveguides. From an investigation of equation (12) above, the inhomogeneity manifests itself in two ways, viz. (a) in the term $\left(\omega^{2} \mu \underset{\sim}{E}\right)$ and (b) $\left.\nabla \underset{\sim}{E} \cdot \nabla \varepsilon\right)$ and $\nabla(\ln \mu) \times \nabla \times \underset{\sim}{\mathrm{E}}$. When the dielectric permittivity and magnetic permeability vary much more slowly than the wavelength of light the latter terms may be ignored in comparison with the former $[12,13]$, and equation (12) then reduces to the Helmholtz equation

$$
\left[\nabla^{2}+k^{2}\right] \underset{\sim}{E}=0,
$$

and

$$
\mathrm{k}=\omega(\mu \varepsilon)^{\frac{1}{2}}=\frac{2 \pi}{\lambda}
$$

is the magnitude of the propagation vector, $\lambda$ being the wavelength of light in the medium in question. We can determine the higher order corrections to the electromagnetic by an iteration procedure, demonstrated in Appendix B. 
If we choose a co-ordinate system in which the z-axis lies in the direction of the waveguide axis, then provided the waveguide is translationally invariant in the axial direction, the field $\underset{\sim}{\mathbb{E}}$ can be

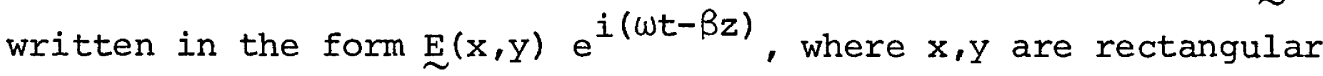
co-ordinates in the transverse plane and $\beta$ is the $z$-component of the wave vector [14]. The minus sign is chosen in the exponent to give a wave travelling in the positive $z$-direction (a forward wave) when the real part of $\beta$ is positive.

Substituting this form for the electric field in equation (13) we obtain the equation

$$
\left[\nabla_{t}^{2}+\left(k^{2}-\beta^{2}\right)\right] \underset{\sim}{E}=0
$$

where $\underset{\sim}{\nabla_{t}^{2}}$ is the transverse part of the Laplacian operator. An identical equation is obtained for $\mathrm{H}$.

In rectangular co-ordinates, equation (15) separates so that each component of $\underset{\sim}{\mathrm{E}}$ (or $\underset{\sim}{\mathrm{H}}$ ) satisfies the scalar Helmholtz equation, $\nabla_{t}^{2}$ being replaced by the scalar operator

$$
\nabla_{t}^{2}=\frac{\partial^{2}}{\partial x^{2}}+\frac{\partial^{2}}{\partial y^{2}}
$$

In the cylindrical polar co-ordinate system more appropriate to our problem, the equations for the transverse components remain coupled, however, and it is only the z-component which satisfies the scalar Helmholtz equation, with

$$
\nabla_{t}^{2}=\frac{\partial^{2}}{\partial r^{2}}+\frac{1}{r} \frac{\partial}{\partial r}+\frac{1}{r^{2}} \frac{\partial^{2}}{\partial \phi^{2}}
$$

But the transverse components can be derived directly from the longitudinal ( $z$ ) components.

To see this we write

and

$$
\begin{aligned}
& \underset{\sim}{\nabla}=\underline{\sim}_{t}+\frac{\partial}{\partial z} \hat{z},
\end{aligned}
$$

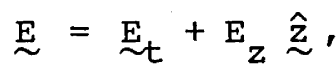

$$
\underset{\mathrm{H}}{\sim}=\underset{\mathrm{H}}{\mathrm{H}}+\mathrm{H}_{\mathrm{z}} \underset{\sim}{\stackrel{\mathrm{z}}{\sim}} \text {, }
$$


where $\underset{\sim}{\hat{z}}$ is a unit vector in the $z$-direction and the subscript $t$ again indicates the transverse part. Then substituting in equations (9) and (10) and equating trasnverse components, we find

$$
\begin{aligned}
& -i \omega \mu_{\sim_{t}}={\underset{\sim}{\nabla}}_{t} \times\left(E_{z} \quad \hat{z}\right)+\hat{z} \frac{\partial}{\partial z} \times{\underset{\sim}{t}}_{t} \\
& i \omega \varepsilon{\underset{\sim}{t}}_{t}=\underset{\sim}{\nabla} \times\left(H_{z} \stackrel{\hat{z}}{\sim}\right)+\underset{\sim}{\hat{z}} \frac{\partial}{\partial z} \times \underset{\sim}{H_{t}} \cdot
\end{aligned}
$$

Substituting equation (22) into (21),

$$
k^{2}{\underset{\sim}{H}}_{t}=i \omega \varepsilon\left(\underset{\sim}{\nabla} E_{z} \times \underset{\sim}{\hat{z}}\right)+\underset{\sim}{\nabla}\left(\frac{\partial H_{z}}{\partial z}\right)-\frac{\partial^{2}}{\partial z^{2}} \underset{\sim t}{H} .
$$

Finally, using the fact that $\partial / \partial z=-i \beta$ in equation (23), we have

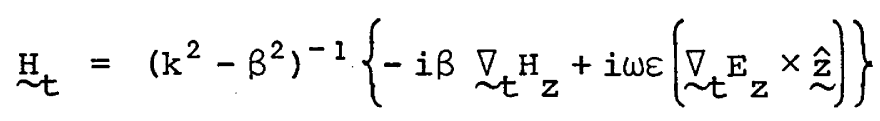

and similarly

$$
\stackrel{E}{\sim}_{t}=\left(k^{2}-\beta^{2}\right)^{-1}\left\{-i \beta \underset{\sim}{\nabla} E_{z}-i \omega \mu\left(\underset{\sim}{\nabla_{t}}{ }_{z} \times \underset{\sim}{\sim}\right)\right\} .
$$

Equations (24) and (25) hold for arbitrary graded-index profiles provided only that $\varepsilon$ and $\mu$ do not vary with $z$.

So in determining the fields propagating along a waveguide, we need only solve the scalar Helmholtz equation for the z-components of the electric and magnetic fields. The remaining components can be found from equations (24) - (25).

The particular system we will be studying in the remainder of this thesis is that shown in fig. 1. The "core" of the waveguide has radius $\rho$, refractive index $n_{1}(r)$ (dielectric permittivity $\varepsilon(r)=\varepsilon_{0} n_{1}^{2}$ ) and magnetic permeability $\mu_{1}$. It is embedded in an infinite "cladding" of uniform refractive index $n_{2}\left(<n_{1}\right)$ and magnetic permeability $\mu_{2} \cdot^{\dagger}$ Both $\varepsilon_{1}$ and $\varepsilon_{2}$ are assumed real so that the system is dissipationless. The effect of this assumption in the study of the practical optical

$+\mu_{1}$ and $\mu_{2}$ are allowed to differ in deriving the modal fields in order to retain symmetry in the expressions for electric and magnetic fields but all calculations will assume that $\mu_{1}=\mu_{2}$. 


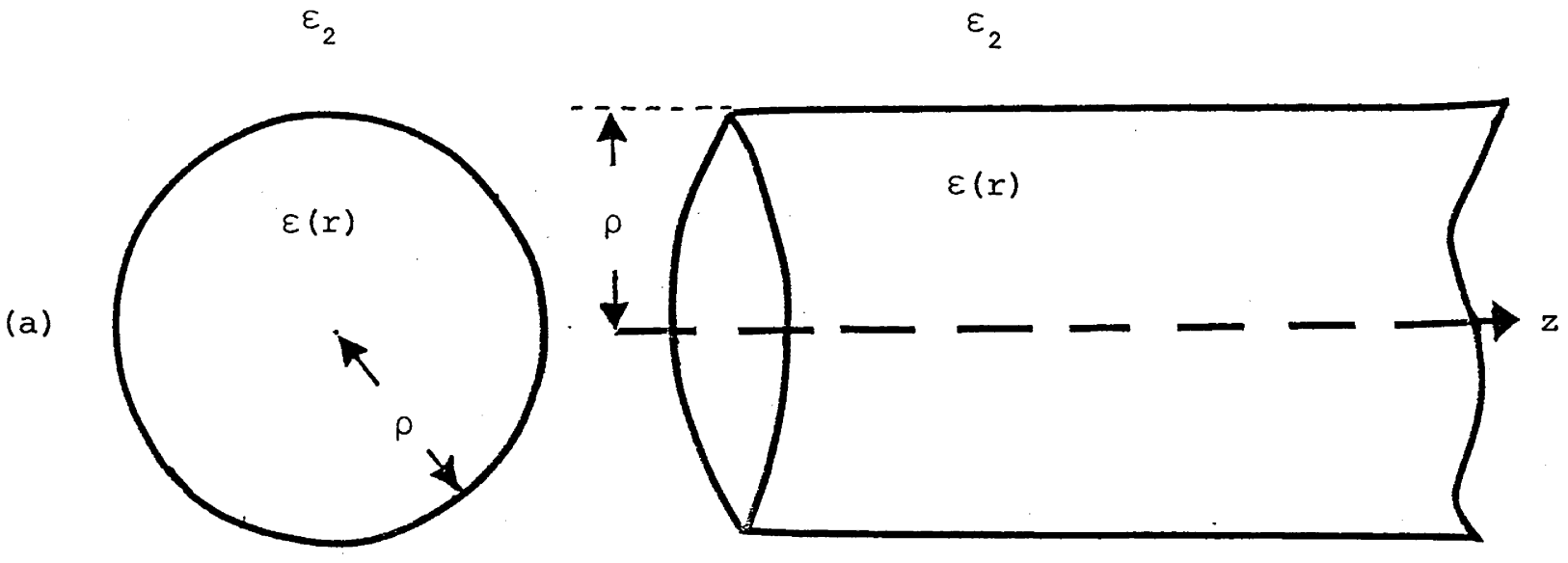

(b)

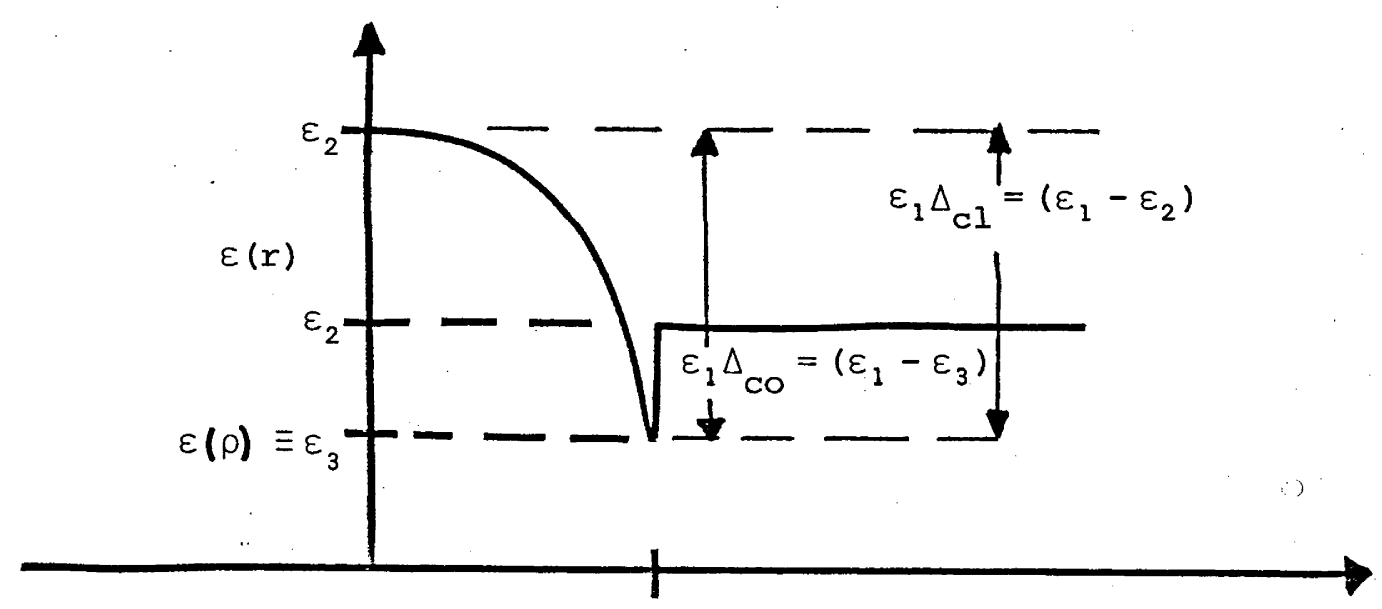

Fig. 1: Circular cylindrical ideal dielectric optical waveguide with an arbitrary core-cladding dielectric difference as defined in fig. $I(b)$. 
fibre has been discussed previously in section 1.3.

In the system with cylindrical symmetry shown in fig. 1, the requirement that the electromagnetic field be a single-valued function of position implies that the solution of the scalar wave equation is of the form

$$
f_{\ell}(r) \exp [i(\omega t-\beta z-\ell \phi)]
$$

where $\ell$ is an integer. The equation satisfied by $\mathrm{f}_{\ell}$, found by substituting equation (26) in equation (15) using equation (17), is then

$$
\frac{d^{2} f_{\ell}}{d r^{2}}+\frac{1}{r} \frac{d f_{\ell}}{d r}+\left\{k^{2}(r)-\beta^{2}-\frac{\ell^{2}}{r^{2}}\right\} f_{\ell}=0,
$$

where

$$
k(r)=k(r)=\omega\left[\mu_{1} \varepsilon(r)\right]^{\frac{1}{2}} \text { in the core, }
$$

and

$$
=k_{2}=\omega\left(\mu_{2} \varepsilon_{2}\right)^{\frac{1}{2}} \quad \text { in the cladding. }
$$

In equations (26) - (27), $\beta$ may be either positive (forward modes) or negative (backward modes), the fields having reflection symmetry about the plane $z=0$. Since we shall be concerned with problems in which a source is placed in this plane with a semi-infinite cylinder extending in the positive $z$ direction, we shall assume from now that $\beta>0$. The magnitude of $\beta$ must be less than $k_{1}$ in order to give well-behaved solutions of equation (27) in the core. For a propagating non-attenuating mode in the dielectric waveguide, the axial component of the phase velocity must be less than that of the external (cladding) medium; therefore, if $\beta>k_{2}$, the field solutions correspond to bound modes, and if $\beta<k_{2}$, the solution corresponds to a radiation mode.

We now have sufficient background to proceed to a discussion of these modes in greater detail. 


\subsection{THE BOUND MODES OF AN IDEAL STRAIGHT DIELECTRIC FIBRE}

From section 2.1 when $\nabla \varepsilon, \nabla \mu$ terms can be ignored, the longitudinal components of the electromagnetic field, $\mathrm{E}_{z}$ and $\mathrm{H}_{z^{\prime}}$, satisfy the scalar wave equation and completely determine the remaining four transverse field components. In this section, we discuss in detail the form of the components of the electromagnetic field for the two region fibre, with an arbitrary smoothly varying refractive index profile in the core. In section 2.2.1, we derive the form of the transverse fields in terms of the longitudinal field components and by matching these fields at the core-cladding interface the eigenvalue equation for the general case of arbitrary differences between core and cladding dielectric permittivities is obtained. In section 2.2.2, we discuss the "weakly guiding" approximation and derive a simpler set of modes and eigenvalue equation for the practical optical fibre based upon this approximation. The initial derivation of the weakly guiding fibre eigenvalue equation closely follows the original formulation by snyder [8].

\subsubsection{The Bound Modes of the Ideal} Straight Dielectric Waveguide

We take $f_{\ell}(r)$ to be the solution of equation (1.27) in the core that is bounded for $r \leqslant \rho$, and the solution in the cladding is chosen to be $\mathrm{H}_{\ell}^{(1)}$ (iWr/ $/$ ) (the Hankel function of the first kind [17]), to satisfy the boundary condition at infinity [10], where $\mathrm{W}$ is defined in equation (5) below. The four transverse field components in cylindrical co-ordinates $r, \phi, z$ can be written from equations (1.23) (1.24) in terms of $\mathrm{E}_{z}$ and $\mathrm{H}_{z}$, giving

$$
\begin{aligned}
& E_{\phi}=\frac{1}{k_{T}^{2}}\left\{-\frac{\beta l}{r} E_{z}+i \omega \mu H_{z}^{\prime}\right\} \\
& E_{r}=-\frac{1}{k_{T}^{2}}\left\{i \beta E_{z}^{\prime}+\frac{\ell \omega \mu}{r} H_{z}\right\} \\
& H_{\phi}=-\frac{1}{k_{T}^{2}}\left\{i \omega \varepsilon_{z}^{\prime}+\frac{\beta l}{r} H_{z}\right\}
\end{aligned}
$$




$$
H_{r}=\frac{1}{k_{T}^{2}}\left\{\frac{l \omega \varepsilon}{r} E_{z}-i \beta H_{z}^{\prime}\right\},
$$

where the ' indicates $d / d r$ (not differentiation with respect to the argument); $\mathrm{k}_{\mathrm{T}}$ is the transverse wavenumber and is

$$
\begin{aligned}
\mathrm{k}_{\mathrm{T}}^{2} & =\frac{\mathrm{U}^{2}(r)}{\rho^{2}}=\mathrm{k}_{0}^{2} \varepsilon(r)-\beta^{2}, & & r \leqslant \rho \\
& =-\frac{\mathrm{W}^{2}}{\rho^{2}}=\mathrm{k}_{0}^{2} \varepsilon_{2}-\beta^{2}, & & r \geqslant \rho
\end{aligned}
$$

$\mathrm{w}^{2} \geqslant 0$ for bound modes; where $\mathrm{k}_{0}$ is the wavenumber in free space and $\rho$ is the radius of the core of the fibre. (We neglect the common factor $e^{i(\omega t-\beta z-\ell \phi)}$ in equations (6) and (7) for conciseness.) $E_{z}$ and $H_{z}$ are

$$
\begin{aligned}
E_{z} & =A_{\ell} f_{\ell}(r), & & r \leqslant \rho \\
& =C_{\ell} H_{\ell}^{(1)}\left(\frac{i W r}{\rho}\right), & & r \geqslant \rho \\
H_{z} & =\left(\frac{\varepsilon(r)}{\mu_{1}}\right)^{\frac{1}{2}} B_{\ell} f_{\ell}(r), & & r \leqslant \rho \\
& =\left(\frac{\varepsilon_{2}}{\mu_{2}}\right)^{\frac{1}{2}} D_{\ell} H_{\ell}^{(1)}\left(\frac{i W r}{\rho}\right), & & r \geqslant \rho
\end{aligned}
$$

where $\mathrm{A}_{\ell}, \mathrm{B}_{\ell}, \mathrm{C}_{\ell}$, and $\mathrm{D}_{\ell}$ are as yet undetermined constants. For dielectric profiles for which $\varepsilon(r)$ is analytic in the core region, an investigation of the indicial equation for the power series solution of the radial wave equation, about $T=0$, readily shows that the two roots of this indicial equation differ by $2 l$ which, from the single-valued nature of the fields, is integral. Therefore, only one solution is finite at the origin [18] and we define this to be $f_{\ell}(r)$. The exact form of the solution $f_{\ell}(r)$ need not be known for this analysis. In fact, only in exceptional cases for well-defined mathematical forms of $\varepsilon(r)$, can $f_{\ell}(r)$ be written in a closed form, and in general, an asymptotic analysis of the radial wave equation is used to provide an approximate solution for $f_{\ell}(r)$. In the following analysis, such asymptotic solutions can be used as long as they are uniformly valid in the core. This restriction, therefore, invalidates the use of the common W.K.B.J. [19] approximate solutions as they are singular at the 
caustics where the electromagnetic field changes from being a propagating wave in the radial direction to an evanescent wave. The use of connection formulae [19] across these caustic regions, implicitly introduces a third dielectric region and thus invalidates the following analysis restricted to two region waveguides. Alternative asymptotic techniques which yield uniformly valid solutions throughout the core are possible $[20,21]$ but will not be discussed further in this thesis.

To satisfy the boundary conditions at the core-cladding interface, we require, in general, both $\mathrm{A}_{\ell}$ and $\mathrm{B}_{\ell}$, the arbitrary constants of the longitudinal electric and magnetic fields, to be non-zero. The presence of both means that the fields in the cylindrical geometry cannot be described as easily as the planar case in which transverse electric and magnetic fields can propagate independently. The general modal field of the dielectric optical fibre consists of a mixture of both transverse electric and magnetic fields and is described as a "hybrid" mode.

By matching the electromagnetic fields at the core-cladding interface, $r=\rho$, via the appropriate boundary conditions on the continuity of the tangential components of the electromagnetic field, results in four simultaneous equations in the four unknowns $A_{\ell}, B_{\ell}, C_{\ell}$ $\mathrm{D}_{\ell}$. These parameters have unique solutions if the eigenvalue equation is satisfied. The eigenvalue equation for the dielectric fibre is (see Appendix A)

$$
\begin{aligned}
& n_{1} n_{2}-k^{2}=0, \\
& n_{j}=\left.\left\{\frac{a_{j} f_{\ell}^{\prime}}{U^{2}(\rho) f_{\ell}}+\frac{H_{\ell}^{(1)^{\prime}}}{w^{2} H_{\ell}^{(1)}}\right\}\right|_{r=\rho}, \text { for } j=1,2
\end{aligned}
$$

where the ' indicates differentiation with respect to $r$, and

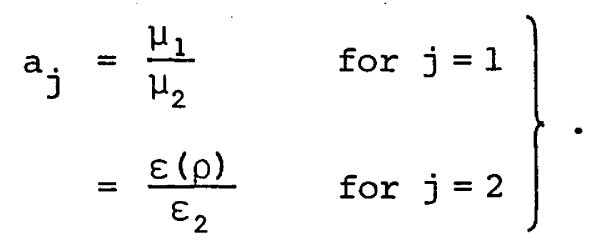

$\varepsilon(\rho)$ is the value of the dielectric permittivity of the core region at 
$r=\rho$ as in fig. 2. $K$ is defined by

$$
\kappa^{2}=\left\{\frac{\beta l}{k_{2} \rho}\right\}^{2} \frac{V^{2}(\rho)}{U^{2}(\rho) W^{2}}
$$

with $\mathrm{k}_{2}=\mathrm{k}_{0}\left(\varepsilon_{2}\right)^{\frac{1}{2}}$, the wavenumber in the cladding, and

$$
V^{2}(\rho)=U^{2}(\rho)+w^{2}
$$

which from equation (5) becomes

$$
V^{2}(\rho)=\left(k_{0} \rho\right)^{2}\left(\varepsilon(\rho)-\varepsilon_{2}\right)
$$

For homogeneous cored dielectric waveguides

$$
V(p) \equiv V^{\prime}
$$

where $\mathrm{V}$ is the well-known normalised frequency of the waveguide [8].

For the solutions for the axial propagation constant, $\beta$, that satisfy the eigenvalue equation, equation (8), the four arbitrary constants are

$$
\begin{aligned}
& C_{\ell}=\left.\left(\frac{f_{\ell}}{H_{\ell}^{(I)}}\right)\right|_{r=\rho}{ }^{A} l \\
& D_{\ell}=\left.\left\{\left(\frac{a_{2}}{a_{l}}\right)^{\frac{1}{2}} \frac{f_{\ell}}{H_{\ell}^{(1)}}\right\}\right|_{r=\rho} A_{\ell}
\end{aligned}
$$

and

$$
B_{\ell}=-\left(\frac{i\left(\frac{a_{2}}{a_{1}}\right)^{\frac{1}{2}} \eta_{2}}{k}\right) A_{\ell}
$$

which from equation (8) is identical to

$$
B_{\ell}=-\left(\frac{i\left(\frac{a_{2}}{a_{1}}\right)^{\frac{1}{2}} k}{n_{1}}\right) A_{\ell} .
$$

For the step-index fibre where $f_{\ell}=J_{\ell}(U r / \rho)$ (where $J_{\ell}$ is the Bessel function [17] of order $l)$ these results agree with those of ref. [7], when the travelling wave (i.e. $e^{-i \ell \phi}$ ) formulation is used. 


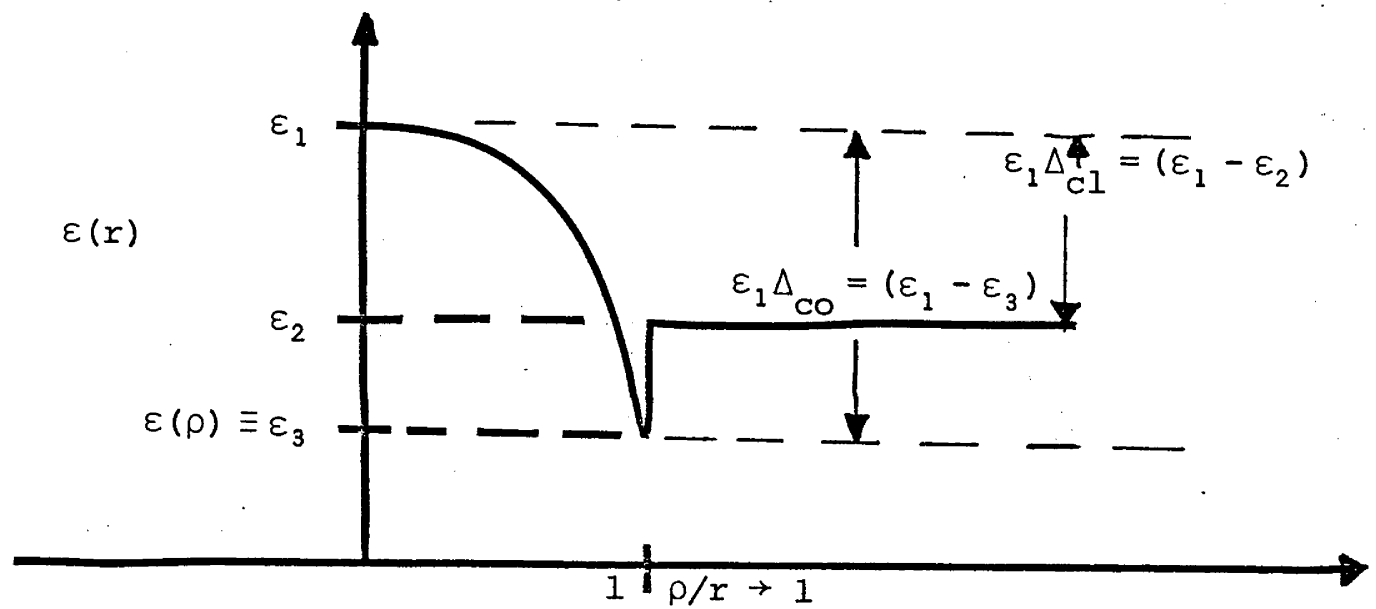

Fig. 2: The dielectric profile of the circular cylindrical dielectric optical fibre. 
Let us investigate the particular case of $K=0$ more closely. This situation occurs when the azimuthal mode number $\ell$.satisfies

$$
\ell \equiv 0
$$

and for

$$
V(\rho)=0
$$

which occurs for dielectric profiles which are continuous across the core-cladding interface, i.e. $\varepsilon(\rho)=\varepsilon_{2}$. When $k=0$, the two arbitrary components $\mathrm{A}_{\ell}$ and $\mathrm{B}_{\ell}$ are independent and one can choose either $\mathrm{A}_{\ell}=0$, $\mathrm{B}_{\ell} \neq 0$ which corresponds to a $\mathrm{TM}$ mode or $\mathrm{A}_{\ell} \neq 0$ and $\mathrm{B}_{\ell}=0$ which corresponds to a $\mathrm{TE}$ mode where $\mathrm{TE}$ (or TM) indicate the electric (or magnetic) field is purely in the transverse plane.

The result for $l=0$ is well known for step-index waveguides and waveguides with walls of infinite conductivity. However, from equation (19) using equation (13), it appears that all modes in a waveguide with a continuous dielectric profile can decouple into the TE and TM modes for all values of the azimuthal mode number. In Appendix 2B this apparent decoupling of the fields for waveguides satisfying equation (19) is investigated and it is shown that this effect is a manifestation of ignoring $\nabla \varepsilon$ terms for these profiles. With this in mind, a hybrid mode consisting of both polarisations will be used, even when equation (19) is satisfied, in order to retain the generality of these modal fields for all graded-index profiles.

The orthogonality of these modes follows directly from the Lorentz Reciprocity Theorem [5] and the normalisation of the modes to unit power follows straightforwardly. However, due to the complex form of this general modal field, we shall not attempt this calculation for the general case, but shall derive the results only for the weakly guiding dielectric fibre modes that will be derived in the next section.

2.2.2 The Weakly Guiding Fibre: $\frac{\varepsilon_{1}}{\varepsilon_{2}} \sim 1$

Due to the increase in intermodal dispersion of a temporal pulse as the dielectric difference between core and cladding increases, 
practical optical fibres employ small dielectric differences to maximise the information carrying capacity. This condition can be stated as

$$
\Delta=\frac{\varepsilon_{1}-\varepsilon_{2}}{\varepsilon_{1}}=\sin ^{2} \theta_{C} \ll 1
$$

where $\varepsilon_{1}, \varepsilon_{2}$ are defined in fig. 2 , and $\theta_{C}$ is the acceptance angle of the waveguide.

Equation (20) allows $\sin \theta_{C}$ to be approximated by

$$
\sin \theta_{C} \sim \theta_{C}
$$

Using this physical constraint, equation (20), in equations (8) - (17) for the amplitude of the field components and eigenvalue euqation, introduces considerable simplification of the expressions for the modal fields.

Since the longitudinal propagation constant, $\beta$, must satisfy

$$
\mathrm{k}_{2}=\mathrm{k}_{0} \varepsilon_{2}^{\frac{1}{2}} \leqslant \beta \leqslant \mathrm{k}_{1}=\mathrm{k}_{0} \varepsilon_{1}^{\frac{1}{2}}
$$

for a bound mode, the weakly guiding fibre approximation, equation (20), has a clear physical interpretation using geometric optics. By defining an angle $\theta_{z}$ to the z-axis as in fig. 1.5 such that

$$
\beta=k_{1} \cos \theta_{z} \text {, }
$$

we see that bound modes of the weakly guiding fibre correspond to wave vectors with

$$
\theta_{\mathrm{z}} \leqslant \theta_{\mathrm{c}} \ll 1
$$

and if we interpret the direction of the wave vector as the ray direction of a geometric optics ray, the "bound rays" are paraxially directed. Thus, the weakly guiding approximation is also known as the paraxial ray approximation.

In the practical optical fibre $\mu_{1}=\mu_{2}$ as well as $\varepsilon_{1} / \varepsilon_{2} \sim 1$. Perturbing the eigenvalue equation about $\Delta \varepsilon=0$, we readily see that the zeroth order approximation for the eigenvalue equation (8) becomes 


$$
n_{0}=\mp \kappa_{0}
$$

where

$$
\begin{aligned}
\eta_{0} & =\eta_{1}=\eta_{2} \quad \text { with } a_{j}=1 \text { for } j=1,2 \\
& =\left.\left\{\frac{1}{U^{2}(r)} \frac{f_{\ell}^{\prime}}{f_{\ell}}+\frac{1}{w^{2}} \frac{H_{\ell}^{(1)^{\prime}}}{H_{\ell}^{(1)}}\right\}\right|_{r=\rho},
\end{aligned}
$$

and $k_{0}$ from equation (11), using the paraxial approximation of $\beta / k_{2} \sim 1$, is

$$
K_{0}=\frac{\ell}{\rho} \frac{V^{2}(\rho)}{U^{2}(\rho) W^{2}} .
$$

In conventional parlance for the step-index fibre, the modes corresponding to the $\mp$ signs are referred to as the $\mathrm{HE}_{\ell}$ and $\mathrm{EH}_{\ell}$ modes with the upper sign for the HE modes and the lower for the EH modes. This convention, established by Beam et al. [24], refers to the dominant axial component of the electromagnetic field. The $\mathrm{EH}_{\ell}$ fields are those for which the magnitude of the z-component of the electric field is greater than the corresponding component of the magnetic and vice versa for the $\mathrm{HE}_{\ell}$ modes.

In the weakly guiding fibre approximation equation (16) or (17) for the inter-relationship of the magnitudes of two polarisations defined in equations (6) and (7) become

$$
\mathrm{B}_{\ell}= \pm \mathrm{iA}_{\ell}
$$

In the following analysis the upper sign is taken as referring to the $\mathrm{HE}$ modes and the lower sign for the $\mathrm{EH}$ modes following the extension of the nomenclature of Beam to weakly guiding dielectric waveguides [7]. From equation (28) it can be seen that the raison d'étre for the nomenclature becomes shallow for the weakly guiding fibre.

Upon substitution of equation (28) into equations (1) - (4) via (6) and (7), using the paraxial approximation, the modal electromagnetic fields for the weakly guiding fibre become (excluding the common factor $\exp \{i(\omega t-\beta z-\ell \phi)\})$, 


$$
\begin{aligned}
& E_{z}=A_{\ell}{ }^{F} \ell^{(r)} \\
& E_{\phi}={ }^{A} \ell^{G} \ell^{\mp}(r) \\
& E_{r}= \pm i E_{\phi}
\end{aligned}
$$

and the complex magnetic field in the travelling wave form, viz. ( $e^{-i \ell \phi}$ ) becomes

$$
\underset{\sim}{H}= \pm i\left(\frac{\varepsilon_{1}}{\mu}\right)^{\frac{1}{2}} \stackrel{\mathrm{E}}{\sim},
$$

where the radial variation of the transverse fields $G_{\ell}^{\mp}(r)$ is defined by

$$
G_{\ell}^{\mp}(r)=\frac{k_{1}}{k_{T}^{2}}\left\{\mp F_{\ell}^{\prime}(r)-\frac{\ell}{r} F_{\ell}(r)\right\} .
$$

(The superscript $\mp$ on $G_{\ell}^{\mp}(r)$ refer to the $\mathrm{HE}$ and $\mathrm{EH}$ modes respectively.) $k_{1}$ is the wavenumber at the axis of the waveguide, defined in equation (22). We have consistently used the weak guidance approximation throughout equations (29) - (33).

$F_{\ell}(r)$ in equation (33), used to represent the field in both the core and cladding regions, is defined by

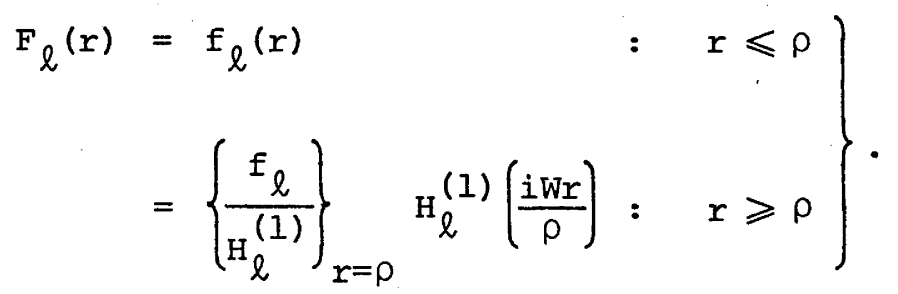

Furthermore, upon investigation of equations (30)-(33) we find that the transverse field components are related by

$$
\underset{\mathrm{T}}{\mathrm{H}_{\mathrm{T}}}=\left(\frac{\varepsilon_{1}}{\mu}\right)^{\frac{1}{2}} \underset{\sim}{\hat{z}} \times{\underset{\mathrm{T}}{\mathrm{E}}} .
$$

where $\underset{\sim}{\hat{z}}$ is the unit vector in the z-direction (along the fibre axis and the subscript $T$ refers to the transverse components.

In the weakly guiding dielectric fibre, the bound mode field components which are dominant are the transverse fields. However, our 
derivation of the field components expresses the transverse fields in terms of the longitudinal field. It would be preferable to have the components expressed in terms of the transverse fields. Such a study has been used by Kurtz and Streifer [22] for more complicated longitudinal field solutions. Substituting $G_{\ell}^{\mp}(r)$ into the radial wave equation, equation (1.27), (or by using equations (1.3-6)) results in a similar equation to equation (33) for $f_{\ell}(r)$ in terms of $G_{\ell}^{\mp}(r)$ and we find (ignoring terms in $\mathrm{d} \varepsilon / \mathrm{d} r$ and higher order)

$$
F_{\ell}(r)=\frac{1}{k_{1}}\left\{ \pm\left(G_{\ell}^{\mp}\right)^{\prime}-\left(\frac{\ell \mp 1}{r}\right) G_{\ell}^{\mp}\right\} .
$$

Using equation (36) together with equation (33), the transverse wave function $G_{\ell}^{\mp}(r)$ is found to satisfy the second order differential equation

$$
\left\{\frac{1}{r} \frac{d}{d r}\left(r \frac{d}{d r}\right)+\left(k_{T}^{2}(r)-\frac{(\ell \mp 1)}{r^{2}}\right)\right\} G_{\ell}^{\mp}=0,
$$

again ignoring all terms in $d \varepsilon / d r$ and higher.

Comparing equation (37) with the radial wave equation, equation(1.27), $G_{\ell}^{\mp}(r)$ satisfies an identical equation with " $\ell$ ", the azimuthal mode number replaced by $(\ell \mp 1) " \cdot G_{\ell}^{\mp}(r)$ is known as the "scalar optical field" [22], which from equation (37) can now be written as

$$
\left.\begin{array}{rlrl}
G_{\ell}^{\mp}(r) & =-N_{\ell \mp 1} f_{\ell \mp 1}\left(r, \beta_{\ell}\right) & \text { for } r<\rho \\
& =-M_{\ell \mp 1} H_{\ell \mp 1}^{(1)}\left(\frac{i W r}{\rho}\right) & \text { for } r>\rho
\end{array}\right\},
$$

where $\mathrm{N}_{\ell \mp 1}, \mathrm{M}_{\ell \mp 1}$ are both constants which must be chosen so that the transverse fields satisfy the boundary conditions at $r=\rho$. Thus, we find

$$
\mathrm{N}_{\ell \mp I}=+\left(\Phi_{\ell \mp l}\right)^{-1} \mathrm{M}_{\ell \mp l}
$$

where

$$
\Phi_{p}=\left(\frac{f_{p}(r)}{H_{p}^{(1)}(i W)}\right)_{r=\rho} \text {. }
$$


Using the recurrence relations for cylinder functions [17] and equation (34), within the weak guidance approximation, ${ }^{M_{\ell \mp 1}}$ can be written as

$$
M_{\ell \mp 1}=+\left(\frac{k_{1} \rho}{i W}\right) \Phi_{\ell}>0,
$$

where $\Phi_{\ell}$ is defined in equation (40). Using equation (38) in (36) we find that the radial variations of the longitudinal fields can now be written as

$$
f_{\ell}\left(r, \beta_{\ell}\right)=-\frac{N_{\ell \mp 1}}{k_{1}}\left\{ \pm f_{l \mp 1}^{\prime}\left(r, \beta_{\ell}\right)-\frac{(\ell \mp 1)}{r} f_{\ell \mp 1}\left(r, \beta_{\ell}\right)\right\},
$$

where we have explicitly included the dependence of the functions, $f_{\ell}(r)$, on $\beta_{\ell}$, the eigenvalue, so that equation (42) is not confused with ordinary recurrence relations for the functions.

Using the above relationships between the longitudinal and transverse field components we can now write the modal electromagnetic fields defined in equations (29) - (34) in a simpler form. In the fields presented below, we have extended the analysis to include the modes with azimuthal mode number $l$, defined in the $\exp \{-i \ell \phi\}$ variation of the fields, to include $\ell \leqslant 0$, so that now the modal fields in the waveguide can be written as, ${ }^{\dagger}$ for $r \leqslant \rho$

and

$$
\begin{aligned}
& E_{z}=-\frac{|\ell|}{\ell} A_{|\ell| \mp 1}\left({ }^{N}|\ell| \mp 1 f_{|\ell| \mp 1}(\rho)\right)^{-1} f_{|\ell|}(r) e^{-i \ell \phi} \\
& E_{\phi}={ }_{|\ell| \mp 1}\left(f_{|\ell| \mp 1}(r) / f_{|\ell| \mp 1}(\rho)\right) e^{-i \ell \phi}
\end{aligned}
$$

$$
E_{r}=i \frac{|l|}{\ell} E_{\phi}
$$

and the complex magnetic field, when written in this azimuthal travelling wave form satisfies

\footnotetext{
+ The modulus signs on the subscripts of the functions $f_{\ell}(r)$ etc. is necessary, since only $l^{2}$ appears in the radial wave equation, equation (1.27). From now on however, for the sake of conciseness, we will omit these signs from the order of the function.
} 


$$
\underset{\sim}{\mathrm{H}}= \pm \frac{|\ell|}{\ell} i\left(\frac{\varepsilon_{1}}{\mu}\right)^{\frac{1}{2}} \stackrel{\mathrm{E}}{\sim},
$$

where we have normalised the transverse electric field to unity at the core-cladding boundary, $r=\rho$. As usual the $\mp$ signs refer to the $\left\{\begin{array}{l}\mathrm{HE} \\ \mathrm{EH}\end{array}\right\}$ modes respectively. $A_{\ell \mp 1}$ ' the power normalisation coefficient is related to $A_{\ell}$ defined in equations (29) - (34) by

$$
A_{\ell \mp 1}=N_{\ell \mp 1} A_{\ell}
$$

where $\mathrm{N}_{\ell \mp I}$ is defined by equations (39) and (41). The sign $\mid$ denotes the modulus of the number inside. In the cladding $r \geqslant \rho$, the modal fields are

$$
E_{z}=-\frac{|\ell|}{\ell} A_{|\ell| \mp 1}\left\{\frac{i W}{k_{2} \rho} H_{|\ell|}^{(1)}\left(i W \frac{r}{\rho}\right) / H_{|\ell| \mp 1}^{(1)}(i W)\right\} e^{-i \ell \phi}
$$

and

$$
E_{\phi}=A_{|\ell| \mp_{1}}\left(H^{(1)}|\ell| \mp_{1}\left(\frac{i W r}{\rho}\right) / H^{(1)}|\ell| \mp_{1}(i W)\right) e^{-i \ell \phi}
$$

with all other field components obtained from equations (47)-(48) by application of the relations of equations (45)-(46). These fields are correct to ignoring all terms in $\mathrm{d} \varepsilon / \mathrm{dr}$. Higher order corrections to these fields and the eigenvalue equation can be developed using the formulation of Appendix 2B.

In this travelling wave form we can observe that these results agree with those derived by Marcuse for the step-index weakly guiding fibre. The standing wave forms, viz. $\cos \ell \phi, \sin \ell \phi$ forms, are obtained simply by addition and subtraction of the two travelling wave modal fields.

Either by direct substitution of equations (33) and (42) into the eigenvalue equation, equation (25), or by the observation from (42), we can show that all boundary conditions can be satisfied by the condition

$$
\left.\frac{f_{l \mp 1}^{\prime}}{f_{\ell \mp 1}}\right|_{r=\rho}=\frac{\mathrm{H}_{\ell \mp 1}^{(1)}(i w)}{\mathrm{H}_{\ell \mp 1}(i w)},
$$


where as usual the ' indicates $d / d r$.

This simple form of the eigenvalue equation for the modes with azimuthal mode number $l$, allows easy computation of the cutoff values of a mode, exactly analogous to Suitzer's treatment for the cutoff values for the step-index fibre modes. It is immediately apparent from equation (49), that the $\mathrm{HE}_{\ell}$ and $\mathrm{EH}_{\ell-2}$ modes satisfy identical eigenvalue equations so that the degeneracy of these mode pairs is the same as in the step-index weakly guiding fibre.

Equation (49) is identical to the eigenvalue equation derived by the "scalar" approximation recently used by Arnaud [24] for stepindex waveguides, or from the analysis of graded-index fibres by okoshi and okamoto [25], and more recently Hashimoto et al. for cladded parabolic profiles [26].

To conclude our discussion on the modes of the weakly guiding fibre, we must determine the formulae for the normalisation coefficient $A^{\prime}{ }^{\prime} \mp 1$ (defined below equation (46)), defined to be the arbitrary constant that yields unit power propagating in the mode with azimuthal mode number $l$.

The conventional power orthogonality between electromagnetic modes propagating in the same axial direction, with fields defined by

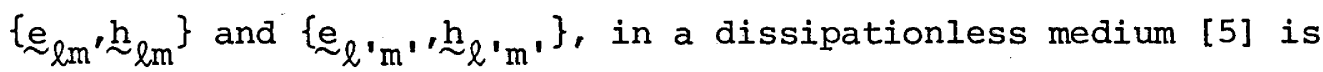

$$
\frac{1}{2} \int_{A_{\infty}} e_{\ell m} \times \underset{\sim}{h} \ell^{\prime} m^{\prime} \cdot \underset{\sim}{\underline{z}} \mathrm{dA}=\delta_{\ell \ell^{\prime}} \delta_{\mathrm{mm}}, \cdot
$$

where the $\delta$ 's are the usual Krönecker delta and $A_{\infty}$ is the infinite cross-sectional plane. (The additional subscripts $m$ and $m$ ' refer to the mth (or $m^{\prime} t h$ ) solution of the eigenvalue equation for the mode with azimuthal mode number $\ell$ (or $\ell$ ').)

Using equation (35) we can now write equation (50) as

$$
\frac{1}{2}\left(\frac{\varepsilon_{1}}{\mu}\right)^{\frac{1}{2}} \int_{A_{\infty}} \cdot \stackrel{e}{T}_{\ell m}^{T} \cdot\left(e_{\ell m}^{T}\right)^{*} d A=1
$$

Substituting for $e_{\ell m}^{\prime}$ from equations (43) - (48) equation (51) 
becomes

$$
\left(\mathrm{A}_{\ell \mp 1, \mathrm{~m}}\right)^{-2}=\left(\frac{\varepsilon_{1}}{\mu}\right)^{\frac{1}{2}} \int_{\mathrm{A}_{\infty}}\left|\mathrm{E}_{\phi}\right|^{2} \mathrm{dA},
$$

which upon conversion of the integral to cylindrical polar co-ordinates can be written as

$$
\left(A_{l \mp 1, m}\right)^{-2}=2 \pi\left(\frac{\varepsilon}{\mu}\right)^{\frac{1}{2}} \int_{0}^{\infty} r\left|F_{\ell \mp I}(r)\right|^{2} d r,
$$

where we have used $\mathrm{F}_{\ell \mp l}(r)$ to represent both the core and cladding radial variations of the transverse fields, where

$$
\left.\begin{array}{rl}
F_{\ell \mp 1}(r)=f_{\ell \mp 1}(r) / f_{\ell \mp 1}(\rho) \quad: \quad r \leqslant \rho \\
=H_{\ell \mp 1}^{(I)}(i W r / \rho) / H_{\ell \mp 1}^{(1)}(i W): & r \geqslant \rho
\end{array}\right\} .
$$

We have now derived a very simple set of modes for graded index fibres, defined in equations (43) - (54), where in the expressions for the fields the common factor $\exp \{i(\omega t-\beta z-\ell \phi)\}$ has been omitted.

\subsection{DISCUSSION}

In the previous section we derived simple forms for the modal fields of a wide range of dielectric profiles of the core of a circularly symmetric cylinder, within the restriction of ignoring all terms in $\mathrm{d} \varepsilon / \mathrm{dr}$. This derivation also provides an alternative derivation of the modes of the weakly guiding step-index fibre [8] without resorting to the recurrence relations for the Bessel functions. This simple set of modal fields allows calculation of most radiative loss processes with the same degree of ease as the corresponding calculations for the step-index fibre. Such simple, uniformly applicable forms, allows for Coupled Mode Theory studies of bound mode coupling to be easily extended into a wide range of dielectric profiles.

In the derivation of equations (2.43)-(2.48) for the modal fields we have employed the azimuthal travelling wave formulation via 
azimuthal variations of the form exp $\{-i l \phi\}$. The difference between the modes for $l>0$ and $l<0$ arise only from the equations for the transverse field equations (2.1)-(2.4). The order of the functions is unaffected by this change of sign since only $l^{2}$ appears in equation (1.27). Similarly replacing $(\ell)$ by $(-\ell)$ does not alter the eigenvalues, as can be seen from the form of equation (2.8). By adding and subtracting these two degenerate travelling wave modes we can obtain the usual standing wave formulation, viz. sin and $\cos \ell \phi$ forms. However, the simple relationships between $\underset{\sim}{\mathrm{E}}$ and $\underset{\sim}{\mathrm{H}}$ in the travelling wave forms, viz. equation (2.46), prove to be extremely useful in some of the more involved calculations that follow and thus will be retained.

This derivation of the modal fields employing the classical approach of matching the fields, derived from the longitudinal field components, yields inter-relationships between the components identical to those obtained by Kurtz and Streifer [22] for continuous dielectric profiles. This latter technique, via the $\nabla \varepsilon$ terms, to derive the relative magnitudes of the longitudinal field components, and from a first inspection would appear to produce modal fields correct to first order in $d \varepsilon / d r$. The justification for the inclusion of these terms is that the transverse fields expressed in terms of the longitudinal fields appear to be singular at the point where $k_{T}=0$, without the $\nabla \varepsilon$ terms included. We have shown that within the approximation of ignoring $\nabla \varepsilon$ terms, such singularities in the transverse fields do not occur. On closer investigation of Kurtz and Streifer's analysis, it is seen that they implicitly use the above approximation of ignoring terms in $\mathrm{d} \varepsilon / \mathrm{dr} .^{+}$ This can be proven simply from an elementary argument based upon the divergence relations for the electromagnetic field as follows:

Using

$$
\nabla \cdot(\varepsilon \underset{\sim}{E})=0
$$

we have

$$
i \beta E_{z}=\nabla_{T} \cdot \underset{\sim}{E_{T}}+\nabla(\underset{\sim}{E} \cdot \nabla \ln \varepsilon)
$$

+ The implicit use of this approximation occurs in the expressions for the transverse fields in terms of the longitudinal field, equations $(29,30)$ of ref. 22. By employing the weak guidance approximation in these expressions all differential operations on these transverse fields ignores terms of the order of $\mathrm{d} \varepsilon / \mathrm{dr}$. 
and from

$$
\nabla \cdot \underset{\sim}{\mathrm{H}}=0
$$

we find

$$
\text { i } \mathrm{H}_{\mathrm{z}}=\nabla_{\mathrm{T}} \cdot{\underset{\mathrm{H}}{\mathrm{T}}}^{\prime}
$$

where $\nabla_{T}$ is the transverse component of $\nabla$, the vector differential operator.

Now if the transverse fields are all proportional to the one scalar field, we have

$$
\nabla \cdot \underset{\mathrm{E}_{\mathrm{T}}}{\mathrm{E}}=c \nabla_{\mathrm{T}} \cdot \stackrel{\mathrm{H}}{\mathrm{T}}
$$

where $\mathrm{c}$ is a constant. For the longitudinal electric and magnetic fields to be of the same functional form, equations (1) and (2) using equation (3) must be consistent. From an investigation of equations (1) and (2), we find such approximations for the fields are consistent only within ignoring all terms in $\mathrm{d} \varepsilon / \mathrm{dr}$. The differential equation for the transverse field function, equation (33) of ref. 22 (or equation (2.37) of the previous section), is the differential equation for the transverse electromagnetic fields only within ignoring all terms in $d \varepsilon / d r$ [28]. Once it is recognised that the analysis is only correct to zeroth order in $d \varepsilon / d r$, the simple modal fields of section 2.2 result.

Together with the above, the analysis of the infinite parabolic profile in ref. 22 using the weak guidance approximation is invalid as this approximation is not possible far from the centre of the profile at $r=0$. As the behaviour of the electromagnetic fields far from the waveguide axis is employed to determine the eigenvalues of the structure, the consequent eigenvalues derived with the weak guidance approximation are of questionable accuracy.

The analysis presented in this chapter does not suffer from such criticisms as the analysis is applicable only to bounded regions over which $d \varepsilon / d r<1$ everywhere, and is totally inapplicable to the infinite profile.

In section 2.1 it was noted that the eigenvalue equation, derived by neglecting terms in $\mathrm{d} \varepsilon / \mathrm{d} r$, indicated that the hybrid modes 
(i.e. $\ell \neq 0$ ) of cylindrical waveguides with continuous dielectric profiles, could decouple into pure $\mathrm{TE}_{\ell}$ and $\mathrm{TM}_{\ell}$ modes. However, upon further investigation (as in Appendix B) into the effect of the $(d \varepsilon / d r$ ) terms, using a perturbation analysis correct to first order in $d \varepsilon / d r$, such decoupling cannot occur and the choice of $\mathrm{TE}_{\ell}$ and $\mathrm{TM}_{\ell}$ modes would result in a singular perturbation analysis.

We see from Appendix B, equation (B.25), that the modes of a fibre with a continuous dielectric profile obey the same relationship between the $\mathrm{TE}_{\ell}$ and $\mathrm{TM}_{\ell}$ components as the hybrid modes of fibres with discontinuous dielectric profiles, i.e. equation (2.28). The zeroth order (in $d \varepsilon / d r$ ) eigenvalue equation for continuous dielectric profiles, equation (2.25) with $V(\rho) \equiv 0$, does provide an interesting result. We observe that for such situations, pairs of $\mathrm{HE}_{\ell}$ and $\mathrm{EH}_{\ell}$ modes become degenerate, although from the analysis we cannot order these pairs of modes. Such extra degeneracies, above the $\mathrm{HE}_{\ell}$ and $\mathrm{EH}_{\ell-2}$ degeneracy of weakly guiding fibre modes, have been observed in numerical studies on graded-index waveguides $[16,27]$. However, extension of the preceding analysis is necessary to determine whether these theoretically predicted degeneracies in weakly guiding fibres correspond to the numerically determined modes.

Appendix B then justifies the choice of the amplitudes of the longitudinal electric and magnetic fields, equation (2.28). The question as to which dielectric inhomogeneity, i.e. the dielectric discontinuity or the gradient of the dielectric, is the dominant first order perturbation cannot be fully answered without resorting to an evaluation of the integrals defined in equation (B.13). (The results of Appendix $B$, for the fields correct to first order in $\Delta$, defined in equation (B.2), can be further simplified, as mentioned in the appendix, thereby simplifying the above comparison. However, as this topic is beyond the scope of this thesis, it will not be considered further.)

The dielectric profiles for which closed form solutions of differential equations of the form

$$
\frac{1}{r} \frac{d}{d r}\left(r \cdot \frac{d \phi}{d r}\right)+k_{r}^{2}(r) \phi=0
$$


(where

$$
\mathrm{k}_{r}^{2}(r)=\mathrm{k}_{\mathrm{T}}^{2}-\frac{l^{2}}{r^{2}}
$$

is the radial wavenumber), are rare and generally are infinite profiles tending to negative infinity (as in the infinite parabolic profile), which are physically unrealistic. ${ }^{\dagger}$

Most theoretical studies on graded index fibres use simple W.K.B.J. asymptotic approximations for solutions of equation (4) above, to circumvent the mathematical complexity. However, these approximate solutions are not uniformly valid throughout the core of the fibre, as, in the vicinity of the "turning points" [19], where $k_{r}^{2}=0$, the solutions diverge. Applying the familiar "connection formulae" across the turning points [19], essentially imposes extra boundaries and therefore the modal relationships of the previous section cannot be readily used, since they are applicable to two region waveguides only. However, for modes whose outer caustic, or turning point, is far from the corecladding interface, at $r=\rho$, the mode should behave as if it were in the infinitely extended medium. With these modes the simple W.K.B.J. solutions should provide good estimations of the fields, provided the conditions for the applicability of the asymptotics are satisfied [21] .

Due to the difficulties involved in differentiation of asymptotic series [21], any modal field components which are formed from the derivatives of other field components estimated by asymptotic techniques should be viewed with some scepticism. Such a difficulty is incumbent on Kurtz and Streifer's results which leads to longitudinal field components which are expressed in terms of the scalar optical field and its derivative. The analysis of section 2.2 circumvents this diffficulty and allows for direct and simple asymptotic representations of the fields from the asymptotic analysis of equation (4) together with equations (2.43) $-(2.48)$.

\footnotetext{
+ Landau and Lifshitz confirm the unphysical nature of such static dielectric permittivities by a thermodynamic argument [29]. In dielectric waveguides, the dielectric permittivity is taken to be the static dielectric permittivity unlike the case of plasma waveguides in which the dielectric permittivity can be less than zero.
} 
We retain the $\mathrm{HE}, \mathrm{EH}$ nomenclature for the modal fields in preference to the transverse field representation, denoted by the LP modes, derived by Gloge for the step-index fibre [30], ${ }^{+}$and more recently extended by Kurtz to graded-index fibres [32] and used exclusively in ref. 31. Although such a modal field solution can be generated for a general class of dielectric profiles of the fibre core, by a linear combination of the $\mathrm{HE}_{\ell}$ and $\mathrm{EH}_{\ell-2}$ modes, this set of "pseudo" modes suffer from major disadvantages. Since the LP mode set is a linear combination of the "proper" modes, i.e. $\mathrm{HE}_{\ell}, \mathrm{EH}_{\ell-2}$ modes (which are only degenerate within the weakly guiding fibre approximation), they are pseudo-stable modes due to the slight difference between the propagation constants, $\Delta B=\beta_{\mathrm{HE}} \ell^{-\beta_{\mathrm{EH}} \mathrm{H}_{-2}}$. Consequently the two real modes of the fibre lose their intimate phase relationship (necessary for the formation of the LP modes) as they propagate along the waveguide. The distance for which the pseudo modes are stable for step-index waveguides is [33]

$$
\frac{z}{\rho} \sim o\left(\frac{\pi}{\theta_{C}^{3}}\right)
$$

where $\theta_{C}$ is the complement of the critical angle, defined in equation (2.20). For practical optical fibres this distance is of the order of millimetres and thus are not sufficiently stable for the description of the practical optical fibre.

Together with the above disadvantage, the LP modes have nonuniform azimuthal intensity distributions, which becomes significant for calculation of modal attenuation coefficients induced by bends in the fibre axis. In such studies, the radiation loss of the LP modes depends critically on the orientation of the mode to the plane of the bend, varying from zero to a finite value $[33,34]$.

\footnotetext{
$\dagger$ The corrected eigenvalue equation, equation 11 of ref. 30 , including corrections to order $\Delta=\left(1-\varepsilon_{2} / \varepsilon_{1}\right)$, is correct. However, this correction cannot be deduced directly from the LP formulation as Gloge indicates in the paper. This is because the LP modes and their boundary conditions are consistent only within the approximation $\varepsilon_{1} / \varepsilon_{2}=1$. To obtain the corrected eigenvalue equation, one requires a perturbation analysis of the exact eigenvalue equation (correct to order $\Delta$ at least) as in Appendix B or appendix B of ref. 30 , or by application of degenerate perturbation theory $[38,39]$.
} 
Before completing this chapter, we shall list the modes of the step-index optical fibre upon which all quantitative discussions will be based.

For the step-index profile, as in figs. $1.4 \mathrm{a}$ and $1.4 \mathrm{c}$, the profile is defined by

$$
\left.\begin{array}{rlr}
\mathrm{n}^{2}(r) & =\mathrm{n}_{1}^{2}=\varepsilon_{0}, & \text { for } r \leqslant \rho \\
& =\mathrm{n}_{2}^{2}=\varepsilon_{0}, & \text { for } r>\rho
\end{array}\right\} .
$$

The solution for the radial wave equation for the longitudinal fields in the core is the Bessel function of order $l$, of the first kind [17],

$$
f_{\ell}(r)=J_{\ell}\left(U \frac{r}{\rho}\right)
$$

and from equation $(2.33)$ the transverse radial wave function $F_{\ell}(r)$ in the core is

$$
\begin{aligned}
G_{\ell}^{\mp}(r) & =-\left(\frac{k_{1} \rho}{U}\right) J_{\ell \mp I}\left(U \frac{r}{\rho}\right), \quad \text { for } r \leqslant \rho \\
& =-\left(\frac{k_{2} \rho}{i W} \frac{J_{\ell}(U)}{H_{\ell}^{(I)}(i W)} \cdot H_{\ell \mp I}\left(i W \frac{r}{\rho}\right) .\right.
\end{aligned}
$$

Using the recurrence relations for the Bessel functions [36] the eigenvalue equation $(2.49)$ becomes

$$
\frac{1}{U} \frac{J_{\ell \mp I}(U)}{J_{\ell}(U)}=\frac{1}{i W} \frac{{ }_{\ell \mp I}(i W)}{H_{\ell}(i W)}
$$

and the fields can then be written, from equations $(2.43)-(2.48)$, as

$$
\begin{aligned}
& E_{z}=A_{\ell \mp I} \frac{U}{k_{2} \rho}\left\{F_{\ell}\left(U \frac{r}{\rho}\right) / J_{\ell \mp I}(U)\right\} \cdot e^{-i \ell \phi} \\
& E_{\phi}=-A_{\ell \mp I}\left\{F_{\ell \mp I}\left(U \frac{r}{\rho}\right) / J_{\ell \mp I}(U)\right\} e^{-i \ell \phi} \\
& E_{r}= \pm i E_{\phi}
\end{aligned}
$$




$$
\underset{\sim}{\mathrm{H}}= \pm i\left(\frac{\varepsilon}{\mu}\right)^{\frac{1}{2}} \underset{\mathrm{E}}{\mathrm{E}}
$$

for $\ell \geqslant 0$, where

$$
\begin{aligned}
F_{p} & =J_{p}\left(U \frac{r}{\rho}\right), & & r \leqslant \rho \\
& =\frac{J_{\ell}^{(U)}}{H_{\ell}^{(l)}(i W)} H_{p}\left(i W \frac{r}{\rho}\right), & & r \geqslant \rho,
\end{aligned}
$$

where $\mathrm{A}_{\ell}$ is the normalisation coefficient defined by equation (2.52). Using standard integrals of Bessel functions

$$
\left(A_{\ell \mp 1}\right)^{-2}=\left(\frac{\varepsilon_{1}}{\mu}\right)^{\frac{1}{2}} \pi \rho^{2}\left(\frac{\mathrm{V}}{\mathrm{U}}\right)^{2} \frac{\mathrm{H}_{\ell}^{(1)} \mathrm{H}_{\ell \mp 2}^{(1)}}{\left(\mathrm{H}_{\ell \mp 1}^{(1)}\right)^{2}}
$$

where the argument of the Hankel functions is (iW) and $W$ and $U$ are defined in equation (2.5). 


\begin{abstract}
APPENDIX A
THE MODAL FIELDS OF AN OPTICAL FIBRE WITH ARBITARY DIELECTRIC PERMITTIVITY PROFILE $\varepsilon_{1}(x)$
\end{abstract}

As was discussed in section 2.1 of this chapter, the electromagnetic field components of the bound modes of a dielectric optical fibre can be determined from the form of the longitudinal components, $E_{\mathbf{z}}$ and $\mathrm{H}_{\mathrm{z}}$, via equations $(1.24)-(1.25)$ yielding

$$
\begin{aligned}
& E_{\phi}=\frac{1}{k_{T}^{2}}\left\{-\frac{\beta \ell}{r} E_{z}+i \omega \mu H_{z}^{\prime}\right\} \\
& E_{r}=-\frac{1}{k_{T}^{2}}\left\{i \beta E_{z}^{\prime}+\frac{l \omega \mu}{r} H_{z}\right\} \\
& H_{\phi}=-\frac{1}{k_{T}^{2}}\left\{i \omega E_{z}^{\prime}+\frac{\beta l}{r} H_{z}\right\} \\
& H_{r}=\frac{1}{k_{T}^{2}}\left\{\frac{l \omega \varepsilon}{r} E_{z}-i \beta H_{z}^{\prime}\right\},
\end{aligned}
$$

where $\mathrm{k}_{\mathrm{T}}$ is defined by equations (2.5)

$$
\begin{aligned}
& E_{z}=\left\{\begin{array}{ll}
A_{\ell} f_{\ell}(r) & r<\rho \\
C_{\ell} H_{\ell}^{(I)}\left(i W \frac{r}{\rho}\right)
\end{array}\right\} e^{i(\omega t-\beta z-\ell \phi),}, r>\rho \\
& H_{z}= \begin{cases}\left(\frac{\varepsilon(r)}{\mu_{1}}\right)^{\frac{1}{2}} B_{\ell} f_{\ell}(r) \\
\left.\left(\frac{\varepsilon_{2}}{\mu_{2}}\right)^{\frac{1}{2}}{ }^{D_{\ell} H_{\ell}^{(I)}\left(i W \frac{r}{\rho}\right)}\right\} e^{i(\omega t-\beta z-\ell \phi),}, r>\rho,\end{cases}
\end{aligned}
$$

where $\mathrm{H}_{\ell}^{(1)}$ is the Hankel function of the first kind [17] and is chosen as the appropriate solution in the cladding to ensure the mode is bound 
to the core, $f_{\ell}(r)$ is the solution of the radial wave equation for the longitudinal components, equation (1.27). The ' indicates differentiation with respect to $r$.

Using the boundary conditions for the components tangential to the interface defined by $r=\rho$ of the electric and magnetic fields yields

$$
\begin{aligned}
& \mathrm{C}_{\ell} \mathrm{H}_{\ell}^{(1)}=\mathrm{A}_{\ell} \mathrm{f}_{\ell}(\rho) \\
& \mathrm{D}_{\ell} \mathrm{H}_{\ell}^{(1)}=\left(\frac{\varepsilon(i), \mu_{2}}{\varepsilon_{2} \mu_{1}}\right)^{\frac{1}{2}} \mathrm{~B}_{\ell} \mathrm{f}_{\ell}(\rho)
\end{aligned}
$$

and using equations (1.28) - (1.29)

$$
\begin{aligned}
\frac{1}{U^{2}(\rho)}\left\{-\frac{\beta \ell}{\rho} A_{\ell} f_{\ell}(\rho)+i k(\rho) B_{\ell} f_{\ell}^{\prime}(\rho)\right\} \\
=-\frac{1}{W^{2}}\left\{-\frac{\beta \ell}{\rho} C_{\ell} H_{\ell}^{(1)}+i k_{2} D_{\ell} H_{\ell}^{(1)}\right\}
\end{aligned}
$$

and

$$
\begin{aligned}
\frac{1}{U^{2}(\rho)}\left\{i k(\rho) A_{\ell} f_{l}^{\prime}\right. & \left.+\frac{\beta l}{\rho} B_{\ell} f_{l}\right\} \\
& =-\frac{\left(\frac{\varepsilon_{2} \mu_{1}}{\varepsilon(\rho) \mu_{2}}\right)^{\frac{1}{2}}}{w^{2}}\left\{i k_{2} C_{\ell}{ }_{l}^{(1) '}+\frac{\beta l}{\rho} D_{\ell}{ }^{H}{ }_{l}^{(1)}\right\} .
\end{aligned}
$$

Substituting from equations $(A .7)-(A .8)$ for $C_{l}$ and $D_{l}$, equations (A.9) - (A.10) become

where

$$
\begin{aligned}
& -\kappa A+i\left(\frac{a_{2}}{a_{1}}\right)^{\frac{1}{2}} \eta_{1} B_{\ell}=0 \\
& +i\left(\frac{a_{1}}{a_{2}}\right)^{\frac{1}{2}} \eta_{2} A_{\ell}+\kappa B_{\ell}=0
\end{aligned}
$$

$$
k=\frac{\beta l}{k_{2} \rho}\left(\frac{V^{2}(\rho)}{U^{2}(\rho) W^{2}}\right)
$$

with $V(\rho)$ defined in equation $(2.12)$, and

$$
n_{j}=\left.\left(a_{j} \frac{f_{\ell}^{\prime}}{U^{2}(\rho) f_{\ell}}+\frac{H_{\ell}^{(1)^{\prime}}}{w^{2} H_{\ell}^{(1)}}\right)\right|_{r=\rho},
$$


where

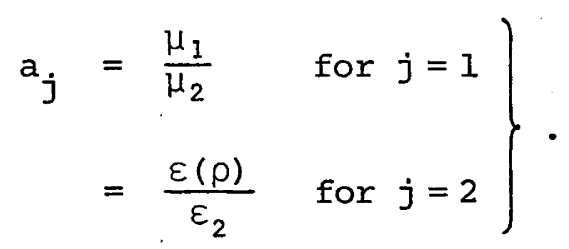

Equations (A.11) - (A.12) constitute a set of linear

homogeneous equations for the unknown parameters $A_{\ell}$, and $B_{l}$ and they admit non-trivial solutions only for the case of the determinant of the coefficients being zero.

This is the eigenvalue equation, with solutions $\beta$ for the longitudinal propagation constant for the bound modes. This eigenvalue equation from (A.11) - (A.12) can be written as

$$
n_{1} n_{2}-\kappa^{2}=0
$$

which when satisfied yields solutions for $A_{\ell}$ and $B_{\ell}$, for $K \neq 0$

$$
B_{\ell}=\left(-\frac{i\left(\frac{a_{2}}{a_{1}}\right)^{\frac{1}{2}} \kappa}{n_{1}}\right) A_{\ell}
$$

or from (A.16)

$$
{ }^{B}=\left(-\frac{i\left(\frac{a_{2}}{a_{1}}\right) n_{2}}{k}\right){ }_{\ell} .
$$

In the particular case of $K=0$, the modes of the optical fibre can be separated into purely transverse electric, TE, corresponding to ${ }^{A} \ell=0$, and transverse magnetic, $T M$, corresponding to $B_{\ell}=0$, modes. The parameter $A_{\ell}$ is determined from the total guided power in the mode [11].

Substitution of equations (A.7) - (A.8) and (A.17) or (A.18) into (A.1) - (A.6) together with the eigenvalue equation provide the explicit expressions for the components of the modal electromagnetic field. 


\section{APPENDIX B \\ ON THE EFFECT OF $\nabla \varepsilon$ TERMS IN INHOMOGENEOUS CIRCULAR CROSS-SECTIONED DIELECTRIC CYLINDERS}

To use the modal electromagnetic fields for cylindrical waveguides derived in section 2.2, with confidence, the effect of the $\nabla \varepsilon$ terms ignored in the analysis should be determined.

Let us explicitly define the dielectric profile as

$$
\left.\begin{array}{rlrl}
\varepsilon(r) & =\varepsilon_{1}(1-\Delta q(r)) & & \text { for } r \leqslant \rho \\
& =\varepsilon_{2} & & \text { for } r>\rho
\end{array}\right\},
$$

where

$$
\begin{aligned}
\Delta_{C l} & =1-\frac{\varepsilon_{3}}{\varepsilon_{1}} \\
q(0) & =0 \\
q(\rho) & =1
\end{aligned}
$$

$q(r)$ is a smoothly varying function, and for generality $\varepsilon_{2} \neq \varepsilon_{3} \equiv \varepsilon(\rho)$.

The longitudinal components of the electromagnetic field obey equations (1.12) which for $\mathrm{E}_{\mathrm{z}}$ can be explicitly written as

$$
\left(\nabla_{\mathrm{T}}^{2}+\mathrm{k}_{\mathrm{T}}^{2}\right) \mathrm{E}_{\mathrm{z}}=\frac{i \beta \varepsilon^{\prime}(r)}{\varepsilon(r)} \mathrm{E}_{r}
$$

and the corresponding equation for $\mathrm{H}_{\mathbf{z}}$, replacing $\varepsilon \rightleftharpoons \mu$ and $\underset{\sim}{\mathrm{E}} \rightleftharpoons \underset{\sim}{\mathrm{H}}$ in equation (1.12), is

$$
\left(\nabla_{\mathrm{T}}^{2}+k_{\mathrm{T}}^{2}\right) \mathrm{H}_{\mathrm{z}}=-i \omega \varepsilon^{\prime}(r) \mathrm{E}_{\phi^{\prime}}
$$

where we have taken $\mu=$ constant and ' indicating $d / d r$, i.e. differentiation with respect to the radial co-ordinate $r$. 
Using the cylindrical symmetry of the fields the $z$ components may be written as

$$
A_{z}(r, \phi, z)=A_{z}(r) e^{i(\omega t-\beta z-\ell \phi)}
$$

where $l$ is integral due to the single-valued nature of the fields. The transverse wave operator $\left(\nabla_{T}^{2}+k_{T}^{2}\right)$ can now be written as

$$
\left(\nabla_{T}^{2}+k_{T}^{2}\right)=\left\{\frac{d^{2}}{d r^{2}}+\frac{1}{r} \frac{d}{d r}+\left(k_{T}^{2}-\frac{\ell^{2}}{r^{2}}\right)\right\} .
$$

For $\Delta<<1$, defined in equation (B.2), the right hand sides of equations (B.3) - (B.4) can be considered as weak coupling terms and a perturbation analysis can be used to determine the higher order corrections to the longitudinal fields. From these longitudinal fields, all other field components can be determined from equations (A.1) - (A.4).

Expanding the solutions of (B.3) - (B.4) in such a perturbation series yields

and

$$
\left.\begin{array}{l}
E_{z}(r)=E_{z}^{(0)}(r)+\Delta E_{z}^{(1)}(r)+\ldots \\
H_{z}(r)=H_{z}^{(0)}(r)+\Delta H_{z}^{(1)}(r)+\ldots
\end{array}\right\},
$$

where the zeroth order fields $\mathrm{H}_{z}^{(0)}, \mathrm{E}_{z}^{(0)}$ satisfy

$$
\left(\nabla_{T}^{2}+k_{T}^{2}\right)\left\{\begin{array}{c}
E_{z}^{(0)}(r) \\
H_{z}^{(0)}(r)
\end{array}\right\}=0
$$

with the transverse wave operator defined in equation (B.6).

Matching coefficients in $\Delta$ we find the first order corrections to these fields, i.e. $\mathrm{E}_{\mathrm{z}}^{(1)}(r)$ and $\mathrm{H}_{\mathrm{z}}^{(1)}(r)$, satisfy

and

$$
\left.\begin{array}{l}
\left(\nabla_{\mathrm{T}}^{2}+k_{\mathrm{T}}^{2}\right) \mathrm{E}_{\mathrm{z}}^{(1)}=-i \beta q^{\prime} \mathrm{E}_{\mathrm{r}}^{(0)} \\
\left(\nabla_{\mathrm{T}}^{2}+\mathrm{k}_{\mathrm{T}}^{2}\right) \mathrm{H}_{\mathrm{z}}^{(1)}=+i \varepsilon_{1} \omega \mathrm{q}^{\prime} \mathrm{E}_{\phi}^{(0)}
\end{array}\right\},
$$

where we have used equation (B.1) for $\varepsilon(r) . \mathrm{E}_{\phi}^{(0)}, \mathrm{E}_{r}^{(0)}$ are the 
transverse electric field components obtained from $\mathrm{E}_{\mathrm{z}}^{(0)},{ }_{\mathrm{H}}^{(0)}$ via equations (A.1) - (A.2) with

$$
\begin{aligned}
& \mathrm{E}_{z}^{(0)}=A_{\ell}^{\prime} \mathrm{f}_{\ell}(r) \\
& { }_{z}^{(0)}=B_{\ell}^{\prime} \mathrm{f}_{\ell}(r)
\end{aligned}
$$

where we have omitted the common factor, $\exp \{i(\omega t-\beta z-\ell \phi)\}$ for conciseness

and

$$
\left.\begin{array}{l}
E_{r}^{(0)}=-\frac{1}{k_{T}^{2}}\left\{i \beta A_{l}^{\prime} f_{l}^{\prime}+\frac{l \omega \mu}{r} B_{l}^{\prime} f_{l}\right\} \\
E_{\phi}^{(0)}=\frac{1}{k_{T}^{2}}\left\{-\frac{\beta l}{r} A_{l}^{\prime} f_{l}+i \omega \mu B_{l}^{\prime} f_{l}^{\prime}\right\}
\end{array}\right\} .
$$

Using equations (B.10) in (B.8), the first order corrections to the longitudinal fields satisfy

$$
\left.\begin{array}{l}
\left(\nabla_{\mathrm{T}}^{2}+k_{\mathrm{T}}^{2}\right) \mathrm{E}_{\mathrm{z}}^{(1)}=\frac{i k_{1}^{2} \mathrm{q}^{\prime}}{k_{\mathrm{T}}^{2}}\left\{i A_{\ell}^{\prime} \mathrm{f}_{\ell}^{\prime}+\frac{\ell}{r}\left(\frac{\mu}{\varepsilon_{1}}\right)^{\frac{1}{2}} \mathrm{~B}_{\ell}^{\prime} \mathrm{f}_{\ell}\right\} \\
\left(\nabla_{\mathrm{T}}^{2}+\mathrm{k}_{\mathrm{T}}^{2}\right) \mathrm{H}_{\mathrm{z}}^{(1)}=\frac{i k_{1}^{2} \mathrm{q}^{\prime}}{\mathrm{k}_{\mathrm{T}}^{2}}\left(\frac{\varepsilon_{1}}{\mu}\right)^{\frac{1}{2}}\left\{-\frac{\ell}{\mathrm{r}} \mathrm{A}_{\ell}^{\prime} \mathrm{f}_{\ell}+i\left(\frac{\mu}{\varepsilon_{1}}\right)^{\frac{1}{2}} \mathrm{~B}_{\ell}^{\prime} \mathrm{f}_{\ell}^{\prime}\right.
\end{array}\right\}
$$

where we have ignored terms in $\mathrm{E}_{r}^{(0)}, \mathrm{E}_{\phi}^{(0)}$, of order $\Delta$ or higher.

$$
\text { Now, using the Green's function, } G_{\ell}\left(r, r_{0}\right) \text { for }\left(\nabla_{T}^{2}+k_{T}^{2}\right) \text {, }
$$
defined in equation (B.6), i.e.

$$
\left\{\frac{d^{2}}{d r^{2}}+\frac{1}{r} \frac{d}{d r}+\left(k_{T}^{2}-\frac{l^{2}}{r^{2}}\right)\right\} G_{\ell}\left(r, r_{0}\right)=\delta\left(r-r_{0}\right)
$$

(where $G_{\ell}\left(r, r_{0}\right)$ satisfies the appropriate boundary conditions at $r=\rho^{\dagger}$ ) with $\delta\left(r_{1}-r_{0}\right)$ the usual Dirac delta function, the first order corrections to the fields become

\footnotetext{
$\dagger$ We could explicitly write [37] $G_{\ell}\left(r, r_{0}\right)=\Sigma a_{m} f_{\ell}\left(r, \beta_{\ell m}\right)$, where $f_{\ell}\left(r, \beta_{\ell m}\right)$ is the solution of the homogeneous transverse wave equation satisfying the boundary conditions, but the following analysis does not require this additional complication.
} 
and

$$
\mathrm{E}_{z}^{(1)}=i \mathrm{k}_{1}^{2}\left\{i \mathrm{~A}_{\ell}^{\prime} \phi_{\ell}^{(1)}+\ell\left(\frac{\mu}{\varepsilon_{1}}\right)^{\frac{1}{2}} \mathrm{~B}_{\ell}^{\prime} \phi_{\ell}^{(2)}\right\}
$$

$$
\left.\mathrm{H}_{\mathrm{z}}^{(1)}=i \mathrm{k}_{1}^{2}\left\{-\ell\left(\frac{\varepsilon_{1}}{\mu}\right)^{\frac{1}{2}} \mathrm{~A}_{\ell}^{\prime} \phi_{\ell}^{(2)}+i \mathrm{~B}_{\ell}^{\prime} \phi_{\ell}^{(1)}\right\}\right\}^{\prime}
$$

where $\phi_{\ell}^{(1)}$ and $\phi_{\ell}^{(2)}$ are defined to be

and

$$
\left.\begin{array}{l}
\phi_{\ell}^{(1)}(r)=\int \frac{1}{k_{T}^{2}\left(r_{0}\right)} q^{\prime}\left(r_{0}\right) f_{\ell}^{\prime}\left(r_{0}\right) G_{\ell}\left(r, r_{0}\right) r_{0} d r_{0} \\
\phi_{\ell}^{(2)}(r)=\int \frac{1}{k_{T}^{2}\left(r_{0}\right)} \frac{q^{\prime}\left(r_{0}\right)}{r_{0}} f_{\ell}\left(r_{0}\right) G_{\ell}\left(r, r_{0}\right) r_{0} d r_{0}
\end{array}\right\} .
$$

Then, using equations (B.7) and (B.9) with equations (B.12), we can write the longitudinal fields correct to first order in $\Delta$ as,

and

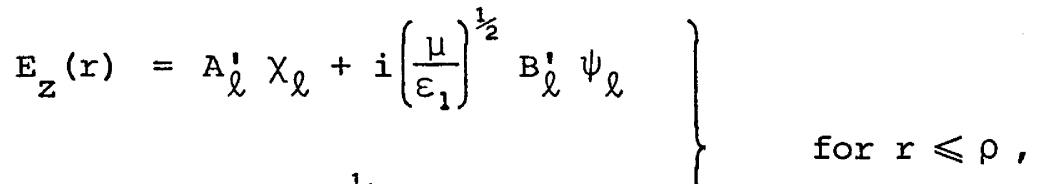

$$
\begin{aligned}
& \mathrm{H}_{z}(r)=-i\left(\frac{\varepsilon_{1}}{\mu}\right)^{\frac{1}{2}} \mathrm{~A}_{\ell}^{\prime} \psi_{\ell}+\mathrm{B}_{\ell}^{\prime} x_{\ell}
\end{aligned}
$$

where

and

$$
\left.\begin{array}{l}
\chi_{\ell}=\mathrm{f}_{\ell}(r)-\Delta \mathrm{k}_{1}^{2} \phi_{\ell}^{(1)} \\
\psi_{\ell}=\Delta \ell \mathrm{k}_{1}^{2} \phi_{\ell}^{(2)}
\end{array}\right\}
$$

and for $r \geqslant \rho$

and

$$
\left.\begin{array}{l}
\mathrm{E}_{z}=\mathrm{C}_{\ell}^{\prime} \mathrm{H}_{\ell}^{(1)}\left(i \mathrm{~W} \frac{r}{\rho}\right) \\
\mathrm{H}_{z}=\mathrm{D}_{\ell}^{\prime} \mathrm{H}_{\ell}^{(1)}\left(i \mathrm{~W} \frac{r}{\rho}\right)
\end{array}\right\} \cdots
$$

Now, using equations (A.1) - (A.4) to obtain the transverse field components, and matching the tangential fields across the boundary, we obtain the inter-relationships between the four unknown constants $A_{\ell}^{\prime}, B_{\ell}^{\prime}, C_{\ell}^{\prime}, D_{\ell}^{\prime}$.

For the z-components, this field matching yields 
and

$$
\begin{aligned}
& C_{\ell}=\left[\frac{1}{H_{\ell}^{(1)}}\left\{A_{l}^{\prime} X_{\ell}+i\left(\frac{\mu}{\varepsilon_{1}}\right)^{\frac{1}{2}} B_{\ell}^{\prime} \psi_{\ell}\right\}\right]_{r=\rho} \\
& \left.D_{\ell}=\left[\frac{1}{H_{\ell}^{(1)}}\left\{-i\left(\frac{\varepsilon_{1}}{\mu}\right)^{\frac{1}{2}} A_{\ell}^{\prime} \psi_{\ell}+B_{\ell}^{\prime} X_{\ell}\right\}\right]_{r=\rho}\right\} .
\end{aligned}
$$

Matching the transverse field components tangential to the boundary at $r=\rho$, using equations (A.1) - (A.4) yields:

For the $\mathrm{E}_{\phi}$ components:

$$
\begin{array}{r}
\frac{1}{U^{2}(\rho)}\left[-\frac{\beta \ell}{\rho}\left\{A_{l}^{\prime} X_{\ell}+i\left(\frac{\mu}{\varepsilon_{1}}\right)^{\frac{1}{2}} B_{l}^{\prime} \psi_{l}\right\}+i k_{1}\left\{-i A_{l}^{\prime} \psi_{l}^{\prime}+\left(\frac{\mu}{\varepsilon_{1}}\right)^{\frac{1}{2}} B_{l}^{\prime} X_{l}^{\prime}\right\}\right] \\
=-\frac{1}{w^{2}}\left\{-\frac{\beta l}{\rho} C_{\ell}^{\prime} H_{\ell}^{(1)}+i \omega \mu D_{l}^{\prime} H_{\ell}^{(1)}\right\},
\end{array}
$$

where $k_{1}=\omega\left(\mu \varepsilon_{1}\right)^{\frac{1}{2}}$ and the argument of the Hankel functions is $i w$, and the ' indicates $d / d r$.

For the $\mathrm{H}_{\phi}$ components :

$$
\begin{aligned}
& -\frac{1}{U^{2}(\phi)}\left\{i \omega \varepsilon_{3}\left(A_{\ell}^{\prime} X_{\ell}^{\prime}+i\left(\frac{\mu}{\varepsilon_{1}}\right)^{\frac{1}{2}} B_{\ell}^{\prime} \psi_{\ell}^{\prime}\right)+\frac{\beta \ell}{\rho}\left(\frac{\varepsilon_{1}}{\mu}\right)^{\frac{1}{2}}\left(-i A_{\ell}^{\prime} \psi_{\ell}+\left(\frac{\mu}{\varepsilon_{1}}\right)^{\frac{1}{2}} B_{\ell}^{\prime} X_{\ell}\right)\right\} \\
& =\frac{1}{W^{2}}\left\{i \omega \varepsilon_{2} C_{\ell}^{\prime} H_{\ell}^{(I) '}+\frac{\beta \ell}{\rho} D_{\ell}^{\prime} H_{\ell}^{(I)}\right\},
\end{aligned}
$$

where $\varepsilon_{3}=\varepsilon(\rho)$ defined in equations (B.1) - (B.2). Using the relations for $C_{\ell}$ and $D_{\ell}$, equations (B.17), in equations (B.18) and (B.19) yield, upon re-arrangement

$$
\begin{aligned}
& A_{\ell}^{\prime}\left\{-\left(\frac{\beta \ell V^{2}(\rho)}{\rho U^{2}(\rho) w^{2}}\right) x_{\ell}+k_{1} \pi_{1}\left(\psi_{l}\right)\right\} \\
& +i\left(\frac{\mu}{\varepsilon_{1}}\right)^{\frac{1}{2}} B_{\ell}^{\prime}\left\{k_{1} \pi_{1}\left(x_{l}\right)-\psi_{\ell}\left(\frac{\beta l V^{2}(\rho)}{\rho U^{2}(\rho) w^{2}}\right)\right\}=0
\end{aligned}
$$

and

$$
\begin{aligned}
& \operatorname{iA}_{\ell}^{\prime}\left\{-\left(\frac{\beta l V^{2}(\rho)}{\rho U^{2}(\rho) w^{2}}\right) \psi_{\ell}+k_{1} \pi_{2}\left(x_{l}\right)\right\} \\
& +\left(\frac{\mu}{\varepsilon_{1}}\right)^{\frac{1}{2}} B_{\ell}^{\prime}\left\{\left(\frac{\beta l V^{2}(\rho)}{\rho U^{2}(\rho) w^{2}}\right) x_{l}-k_{1} \pi_{2}\left(\psi_{l}\right)\right\}=0,
\end{aligned}
$$

where 
with

$$
\pi_{j}(\phi)=\left(\frac{a_{1}^{j} \phi^{\prime}}{U^{2}(\rho)}+\frac{a_{2}^{j} \phi H_{\ell}^{(1)^{\prime}}}{w^{2} H_{\ell}^{(1)}}\right)
$$

and

$$
\left.\begin{array}{rlrl}
a_{i}^{\prime} & =1 & & \text { for } i=1,2 \\
a_{j}^{2} & =\frac{\varepsilon_{3}}{\varepsilon_{1}} & & \text { for } j=1 \\
& =\frac{\varepsilon_{2}}{\varepsilon_{1}} & & \text { for } j=2
\end{array}\right\}
$$

$$
\begin{aligned}
\mathrm{V}^{2}(\rho) & =\mathrm{U}^{2}(\rho)+\mathrm{W}^{2} \\
& =\left(\mathrm{k}_{0} \rho\right)^{2}\left(\varepsilon_{3}-\varepsilon_{2}\right) .
\end{aligned}
$$

Thus, from equations (B.20)-(B.21), we obtain the eigenvalue equation for the modes of the circular cylindrical dielectric waveguide correct to first order in $d \varepsilon / d r$ and $\Delta$. We can readily observe that the continuous profiles, for which $\varepsilon_{3}=\varepsilon(\rho)=\varepsilon_{2}$ such that $V(\rho)=0$, cannot support pure $\mathrm{TE}_{\ell}, \mathrm{TM}_{\ell}$ modes as is suggested from the zeroth order eigenvalue equation, equation (2.8). The relative magnitudes of the $z-$ component fields are as defined in equation (2.28) with equation (2.7), i.e.

$$
\left(\frac{\mu}{\varepsilon_{1}}\right)^{\frac{1}{2}} \mathrm{~B}_{\ell}^{\prime}= \pm i A_{\ell}^{\prime}
$$

and the eigenvalue equation for continuous profiles reduces to

$$
\pi_{1}\left(f_{\ell}\right)=0
$$

correct to ignoring all terms in $d \varepsilon / d r$. Thus, we have now justified the choice of the relative magnitudes for $A_{l}^{\prime}$ and $B_{l}^{\prime}$ as in equation (B.25), even for the continuous dielectric profile, as well as providing the eigenvalue equation and modal fields correct to first order in $\Delta$ and $\mathrm{d} \varepsilon / \mathrm{dr}$.

We could now proceed further with this perturbation analysis, using the relationship between the zeroth order transverse and longitudinal field components, viz. equation (2.33) and (2.42), together with the relationship 


$$
\left(\frac{\mu}{\varepsilon_{1}}\right)^{\frac{1}{2}} B_{\ell}^{\prime}= \pm i A_{\ell}^{\prime}+O(\Delta)
$$

to obtain more manageable forms for the first order corrections to the fields of the dielectric cylinder. However, as only the zeroth fields are to be used in this thesis, such an analysis does not appear to be warranted. 


\section{REFERENCES}

[1] D. Hondros and P. Debye: "Elektromagnetische Wellen und Dielektrischen Drahten", Ann. Phys. 32, 465 (1910).

[2] P.J.B. Clarricoats: "Propagation along bounded and unbounded dielectric rods", Proc. I.E.E., Part C, Monograph No. 409E and 410E (1960).

[3] J.A. Stratton: Electromagnetic Theory (McGraw-Hill, New York, 1941).

[4] S.A. Schelkunoff: Electromagnetic Waves (Van Nostrand Reinhold, New York, 1943).

[5] R.E. Collin: Field Theory of Guided Waves (McGraw-Hill, New York, 1960).

[6] D.G. Kiely: Dielectric Aerials (Methuen \& Co., 1953).

[7] E. Snitzer: "Cylindrical dielectric waveguide modes", J. Opt. Soc. Am. 51, 491 (1961).

[8] A.W. Snyder: "Asymptotic expressions for eigenfunctions and eigenvalues of a dielectric or optical waveguide", I.E.E.E. Trans. MTT-17, 1130 (1969).

[9] G. Biernson and D.J. Kinsley: "Generalised plots of mode patterns in a cylindrical dielectric waveguide applied to the retinal cones", I.E.E.E. Trans. MTT-13, 345 (1965).

[10] J.D. Jackson, Classical Electrodynamics (Wiley, New York, 1962).

[11] D. Marcuse: Light Transmission Optics (Van Nostrand Reinhold, New York, 1972).

[12] D. Marcuse: "The effect of $\nabla n^{2}$ term of the modes of an optical square-law medium", I.E.E.E. J. Quant. Electron. 958, (1973).

[13] M. Matsuhara: "Analysis of electromagnetic-wave modes in lenslike media", J. Opt. Soc. Am. 63, 135 (1973).

[14] R.B. Adler: "Properties of guided waves on inhomogeneous dielectric structures", M.I.T. Research Lab. of Electronics Tech. Report 102, 1949. (Summarised in "Waves in inhomogeneous cylindrical structures", Proc. I.R.E. $\underline{40}, 339$ (1952).) 
[15] P.J.B. Clarricoats and K.B. Chan: "Propagation behaviour of cylindrical dielectric rod waveguides", Proc. I.E.E. 120, 1371 (1973).

[16] P.J.B. Clarricoats: "Theory of optical fibre waveguides: A review" in Progress in Optics 14 (E. Wolf, ed.) (NorthHolland, Amsterdam, 1976).

[17] M. Abramowitz and I. Stegun: Handbook of Mathematical Functions (Dover Press, New York, 1965).

[18] E.T. Whittaker and G.N. Watson: A Course of Modern Analysis (Cambridge Univ. Press, 1927).

[19] L.D. Landau and E.M. Lifshitz: "Electrodynamics of continuous media", Vol. 8 of A Course of Theoretical Physics (Pergamon, Oxford, 1960).

[20] R.E. Langer: "On the asymptotic solutions of ordinary differential equations, with reference to the Stokes' phenomena about a singular point", Trans. Amer. Math. Soc. 37, 397 (1935).

[21] A. Erdélyi: Asymptotic Expansions (Dover Press, New York, 1966).

[22] C.N. Kurtz and W. Streifer: "Guided waves in inhomogeneous focusing media. Part I: Formulation, solution for quadratic inhomogeneity", I.E.E.E. Troans. MTT-17, 11 (1969).

[23] R.E. Beam et al.: North Western Univ. Report A.T.I. 94929, Chapter V (1949).

[24] J.A. Arnaud: "Transverse coupling in fiber optics. Part II: Coupling to mode sinks", B.S.T.J. 54, 675 (1974).

[25] T. Okoshi and K. Okamoto: "Analysis of wave propagation in inhomogeneous optical fibers using a variational method", I.E.E.E. Trans. MTT-22 (11), 938 (1974).

[26] M. Hashimoto, S. Nemoto and T. Makimoto: "Analysis of guided waves along the cladded optical fiber: Parabolic index core and homogeneous cladding", I.E.E.E. Trans. MTT-25 (1), 11 (1977).

[27] P.J.B. Clarricoats and K.B. Chan: "Electromagnetic wave propagation along radially inhomogeneous dielectric cylinders", Electronics Letters, October 1970.

[28] R. Yamada and Y. Inabe: "Guided waves in an optical square law medium", J. Opt. Soc. Am. 64, 964 (1974).

[29] Ref. 19, p.63.

[30] D. Gloge: "Weakly guiding fibers", Appl. Optics 10, 2252 (1970). 
[31] D. Marcuse: Theory of Dielectric Optical Waveguides (Academic Press, New York, 1974).

[32] C.N. Kurtz: "Scalar and vector mode relations in gradient index light pipes", J. Opt. Soc. Am. 65, 1235 (1975).

[33] A.W. Snyder: "Leaky ray theory of optical waveguides of circular cross-section", Appl. Physics 4, 273 (1974).

[34] E.F. Kuester and D.C. Chang: "General theory of surface-wave propagation on a curved optical waveguide of arbitrary crosssection", Univ. of Colorado, Boulder Scient. Rept. No. 11, AFOSR-72-2417 and also in I.E.E.E. Joumal of Quant. Electron. QE-11, 903 (1975).

[35] D. Marcuse: "Curvature loss formula for optical waveguides", J. Opt. Soc. Am. 66, 216 (1976).

[36] Ref. 17, equations 9.1.27.

[37] P.M. Morse and H. Feshbach: Methods of Theoretical Physics, Part I (McGraw-Hill, Tokyo, 1953).

[38] A.W. Snyder: "Mode propagation in optical waveguides", Electron. Lett. 6 (18), September 1970.

[39] A.W. Snyder and W.R. Young: "Modes on optical waveguides", to be published. 
CHAPTER 3

\section{RADIATION FROM IRREGULARITIES IN THE STRAIGHT DIELECTRIC WAVEGUIDE: THE VOLUME CURRENT METHOD}

When a wave travels along a waveguide with imperfections, some of its energy is scattered. A portion of this energy is redistributed among the bound modes while the remainder is radiated away from the waveguide core. Ray optically, we view the radiation loss as due to the ray directions of the angular power distribution of the scattered light, that undergo refraction and electromagnetic tunnelling at the corecladding interface, as in fig. 1. Conventional Coupled Mode Theory for dielectric waveguides, elucidated first by Snyder [1-4] and extended and exhaustively applied by Marcuse [5] is a powerful technique for studying the portion of scattered power redistributed among the bound modes. However, such a technique becomes tedious and complicated to apply for determining the radiation losses. This is due to the complex form of the exact radiation modes of the dielectric waveguide and the difficulties associated with normalisation of these modes, for all but the most simple of planar waveguides.

The exact solution of the radiative losses from imperfections in optical waveguides of circular cross-section, is extremely difficult and always leads to an integral which must be evaluated numerically. For the practical, weakly guiding dielectric waveguide, elementary perturbation solutions can be obtained. Since the dominant type of imperfections in waveguides are structural deformities, the dielectric imperfections associated with these are of the order of core-cladding dielectric difference which is small. This should ensure that the perturbation series analysis rapidly converges.

Even for the special class of weakly guiding dielectric waveguides, the use of Coupled Mode Theory or the dyadic Green's function 


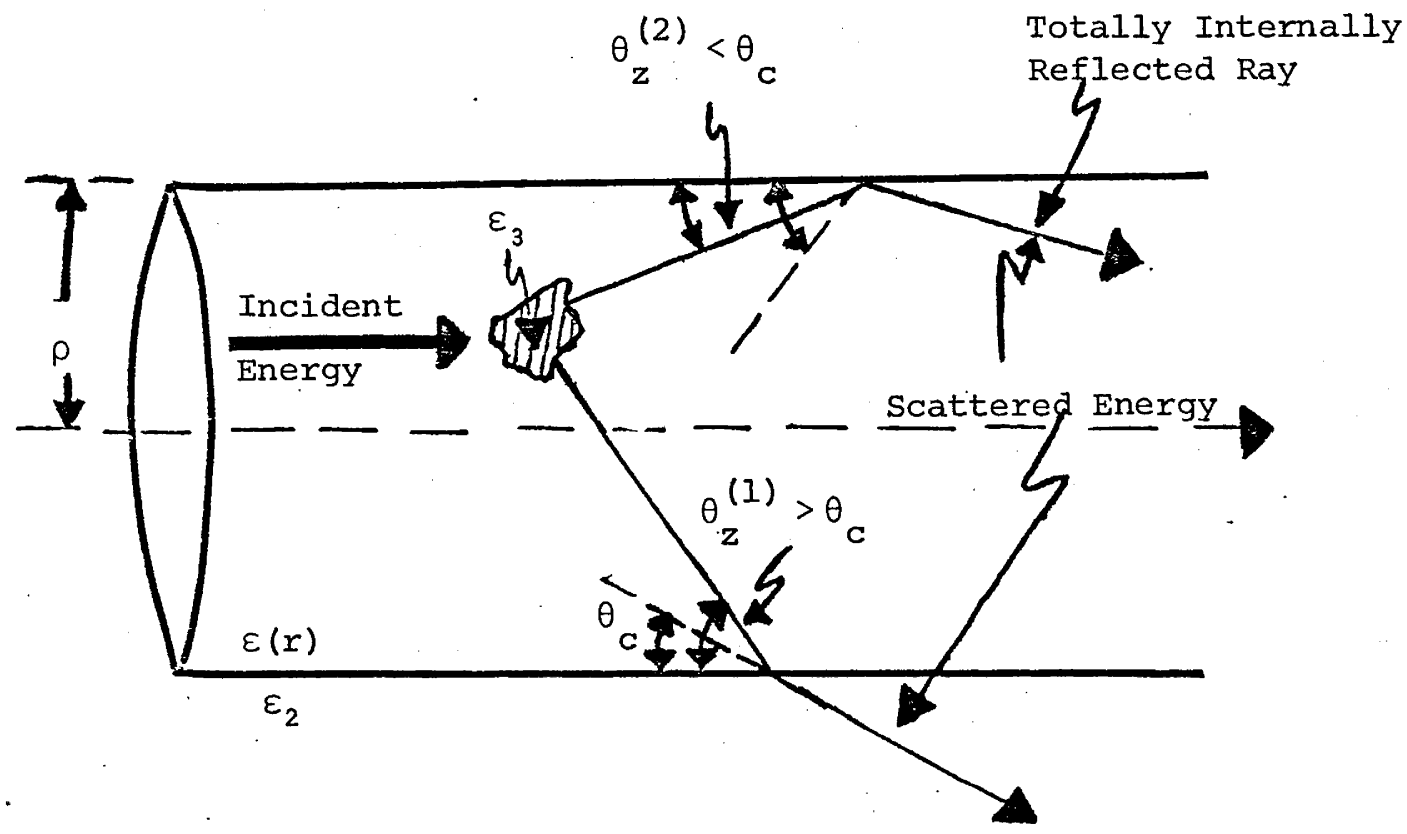

Refracted Ray

Fig. 1: Energy incident on a dielectric irregularity (the shaded region) in the core of the waveguide is scattered. The energy scattered into angles $\theta_{z}<\theta_{C^{\prime}}$ as the ray with $\theta_{z}^{(2)}$ is totally internally reflected. The energy scattered into the rays with angles $\theta_{z}^{(1)}>\theta_{C}$ undergo partial reflection at the core-cladding boundary. 
formulation for radiation losses [6-8], prove to be cumbersome for cylindrical guides. The latter technique becomes both mathematically and numerically intractable, if the imperfections are located off the axis of the fibre.

Snyder proposed a technique for calculation of the radiation losses in weakly guiding fibres that eliminated the need for the complicated analyses of the above techniques [9]. Essentially this technique, now known as the Volume Current Method, treats the imperfections as volume dipole moments, which when excited by an incident field, radiate as if in an unbound homogeneous dielectric with dielectric permittivity, $\varepsilon_{2}$, i.e. the cladding dielectric. More recently, Clarricoats and Chan [10,11] and Rawson [12] have used similar approximations for studies of radiative losses in dielectric waveguides.

This Volume Current Method is an approximation to the dyadic Green's function formulation adopted by Yip [6-8]. By ignoring the core-cladding dielectric difference, the dyadic Green's function for the cylindrical structure degenerates to that of free space [13]. This is a significant simplification since the form of the dyadic Green's function in inhomogeneous cylindrical structures is not known, in general.

For step-index fibres it is obvious that the power scattered by an imperfection, in the core of the waveguide, that is radiated in a direction $\theta_{z}$ to the axis for which

$$
\theta_{z} \leqslant \theta_{c}
$$

does not constitute part of the radiation field of the fibre since it is totally internally reflected at the core-cladding interface. However, since the acceptance angle, $\theta_{C^{\prime}}$ of the fibre is small, only a relatively small proportion of the total scattered power is significantly affected by the core-cladding interface. Therefore, the total power scattered from a diffuse (wide angle) scattering process should be well approximated by the application of this Volume Current Method.

The derivation of the formulae of the Volume Current Method, in Section 1, will follow Snyder's original formulation. This section 
will conclude with a brief discussion of the incorporation of the technique into practical optical fibre studies.

In an attempt to simplify the complex Coupled Mode Theory calculations for radiative losses in dielectric waveguides, the exact radiation modes are often approximated by their "free space" equivalents [14]. In section 2, the equivalence of such an approximate Coupled Mode treatment and the Volume Current Method is demonstrated.

In section 3, we make use of the general modal fields, derived in Chapter 2, in the Volume Current Method, to calculate the radiated power from an incident guided mode for two practically significant loss mechanisms, namely, isolated dielectric irregularities and core-cladding interface roughness. We limit the study to these two mechanisms in order to demonstrate the effectiveness of the Volume Current Method without listing ad nauseum many examples. Quantitative results are provided for the step-index optical fibre together with a discussion of the accuracy of the Volume Current Method.

\subsection{THE VOLUME CURRENT METHOD}

In this section, the formulae for the electromagnetic fields generated by an incident guided field impinging on an irregularity in a dielectric waveguide will be derived from the Volume Current Method. This derivation closely follows that of Snyder's original formulation [9] but extends the treatment to include the effects of irregularities in the magnetic permeability. Although such an extension is not justifiable for optical fibres, it is presented for completeness and because of its possible significance for dielectric waveguides in integrated optics.

If we consider the generalised Maxwell's equations for a monochromatic field (with time variation $e^{i \omega t}$ ), that include the fictitious magnetic current density, $J_{m}$, and charge density $\rho_{m}$, the electromagnetic field equations become [13]

$$
\nabla \times \underset{\sim}{\mathrm{E}}=-{\underset{\sim}{\mathrm{J}}}_{\mathrm{m}}-\mathrm{i} \omega \mu(\underset{\sim}{\underline{r}}) \underset{\sim}{\mathrm{H}}
$$




$$
\begin{aligned}
& \nabla \cdot \underset{\sim}{D}=\rho_{e} \\
& \nabla \times \underset{\sim}{H}={\underset{\sim}{J}}_{e}+i \omega \varepsilon(\underline{\sim}) \underset{\sim}{~} \\
& \nabla \cdot \underset{\sim}{B}=\rho_{m} .
\end{aligned}
$$

To the best of the author's knowledge, these magnetic currents and charges have no physical reality. However, as we shall see, imperfections in the dielectric and magnetic structure of a waveguide can be interpreted as generating electric and magnetic current densities at the location of the irregularity.

In theory, equations (1) - (4) together with the appropriate boundary conditions, allow us to completely determine the electromagnetic fields generated by these current sources. However, closed form solutions for these fields can be obtained only for special distributions of these sources in particular structures where the dielectric permittivity, $\varepsilon(r)$, and the magnetic permeability, $\mu(r)$, have particular, well defined, mathematical forms. Any attempt to construct such a structure, invariably introduces irregularities and imperfections. These departures from the ideal structures can be represented by magnetic and electric current densities, in the ideal structure.

Now writing the field in the perturbed waveguide as the sum of the incident field in the unperturbed waveguide ${\underset{\sim}{0}}_{0}$ and a scattered field due to the perturbation, $\underset{\mathrm{S}^{\prime}}{\mathrm{E}^{\prime}}$ via

$$
\underset{\text { tot }}{\mathrm{E}_{\mathrm{N}}}=\underset{\sim}{\mathrm{E}_{0}}+\underset{\mathrm{S}}{\mathrm{E}}
$$

and rewriting equations (1) and (3) specifically in terms of the ideal structure with $\varepsilon_{0}(\underline{\sim})$ and $\mu_{0}(\underline{r})$, the fields (in the absence of the real sources, i.e. $J_{e}=\underset{\sim m}{J}=0$ ) satisfy,

and

$$
\nabla \times \underset{\sim}{\mathrm{E}}=-\underset{\sim}{J_{\mathrm{S}}^{1}}-i \omega \mu_{0}(\underset{\sim}{(\underline{r})} \underset{\sim}{\mathrm{H}}
$$

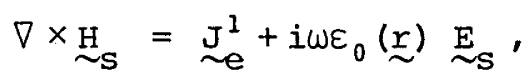

where the pseudo electric and magnetic current densities ${\underset{\sim}{e^{\prime}}}_{\mathrm{J}^{\prime}}^{\mathrm{J}^{1}}$ respectively, are defined by 
where

$$
\begin{aligned}
& {\underset{\sim}{J}}_{e}^{1}=i \omega \Delta \varepsilon(\underline{r}) \underset{\sim}{\stackrel{E}{*}} \\
& \underset{\mathrm{m}}{\mathrm{J}^{l}}=i \omega \Delta \mu(\underset{\sim}{\underline{r}}) \stackrel{\mathrm{H}_{\mathrm{tot}}}{ }
\end{aligned}
$$

$$
\Delta \varepsilon(\underline{\sim})=\varepsilon(\underline{\sim})-\varepsilon_{0}(\underline{r})
$$

and

$$
\Delta \mu(\underset{\sim}{\sim})=\mu(\underline{\sim})-\mu_{0}(\underset{\sim}{\sim}),
$$

where the dielectric permittivity, $\varepsilon(\underline{\sim})$, and magnetic permeability, $\mu(\underline{r})$, refer to the values of these parameters at the position $\underset{\sim}{r}$, in the guide with imperfections.

$$
\text { From equations (5) - (10) we can see that the effect of }
$$
imperfections in the dielectric waveguide can be interpreted as volume densities of electric and magnetic currents which are excited by the electromagnetic fields in the vicinity of the irregularity. These volume currents then radiate energy.

In Appendix A, the electric and magnetic fields that are generated by volume current densities in a waveguide are derived in terms of the dyadic Green's function for the structure.

In terms of this dyadic Green's function $\underset{\sim}{\Gamma}\left(\stackrel{r}{\sim} \stackrel{\underline{\sim}}{\prime}^{\prime}\right)$ the electromagnetic fields of equations (5) and (6) for a weakly guiding dielectric waveguide are

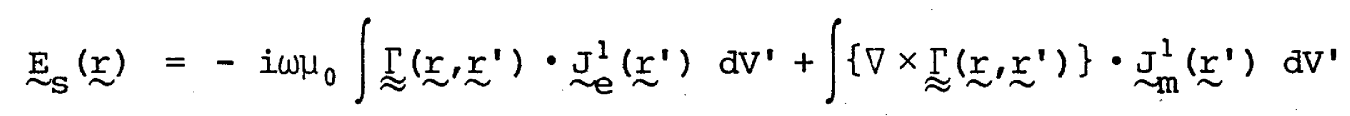
and

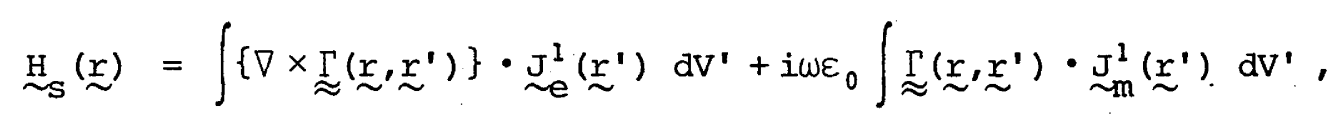

where the $\nabla$ operator refers to the observation point, $\underset{\sim}{r}$.

The integration is over the volume enclosing the current densities $\mathrm{J}_{\mathrm{m}}^{1}, \mathrm{~J}_{\mathrm{e}}^{1}$. (We have used the weakly guiding approximation in equations (11) and (12), by removing the dielectric permittivity and magnetic permeability from the integrand.) In general, the dyadic Green's function cannot be written in a simple closed form.

In particular, for cylindrical waveguides, the general form of 
the Green's function is not known. However, for the specific case of the source situated on the axis of the waveguide with a homogeneous dielectric core, there exists a closed form analytic expression for the Green's function [15]. Angulo and Chang [15] showed that for the particular case of weakly guiding step-index fibres that this dyadic Green's function is the dyadic Green's function of free space to order $\Delta\left(=1-\varepsilon_{2} / \varepsilon_{1}\right)$. Ray optically, the result can be interpreted as a statement that only the radiation that is nearly tangential to the corecladding interface, i.e. $\theta_{z^{\prime}} / \theta_{c} \ll 1$, is affected by the dielectric difference between the core and cladding materials.

Snyder [16] used this result to justify the use of the free space dyadic Green's function, $\underset{\approx}{\mathbb{0}}$, as an approximation to the exact Green's function of the structure, where [13]

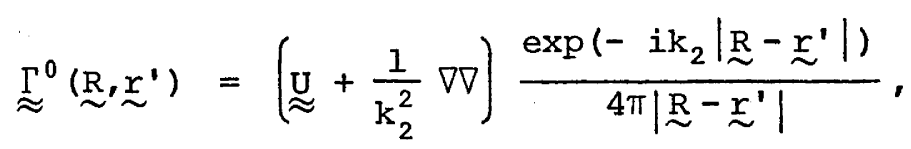

where $k_{2}$ is the wavenumber in the cladding. Until the dyadic Green's function for the cylinder is developed in a mathematical form convenient for investigation, the question of the validity of this approximation for the general dyadic Green's function of a cylinder cannot be adequately answered. In Chapter 4 we attempt such a discussion with respect to step-index fibres.

However, from a geometric optics viewpoint we can readily observe that this approximation becomes less accurate for nonmeridionally directed radiation. The radiation that is coupled into the weakly leaky rays, "whispering gallery"-type rays, are more strongly influenced by the core-cladding interface than meridional rays inclined at the same angle to the waveguide axis, as they are incident at the core-cladding boundary at a greater angle of incidence due to the skewness of the ray (see fig. 2).

This is contrary to the suggestion by Yip [8] that the Volume

\footnotetext{
$\dagger$ The existence of this special case of the dyadic Green's function is the reason that the treatment of radiation loss as in ref. 6-8 is possible for any irregularities at all.
} 

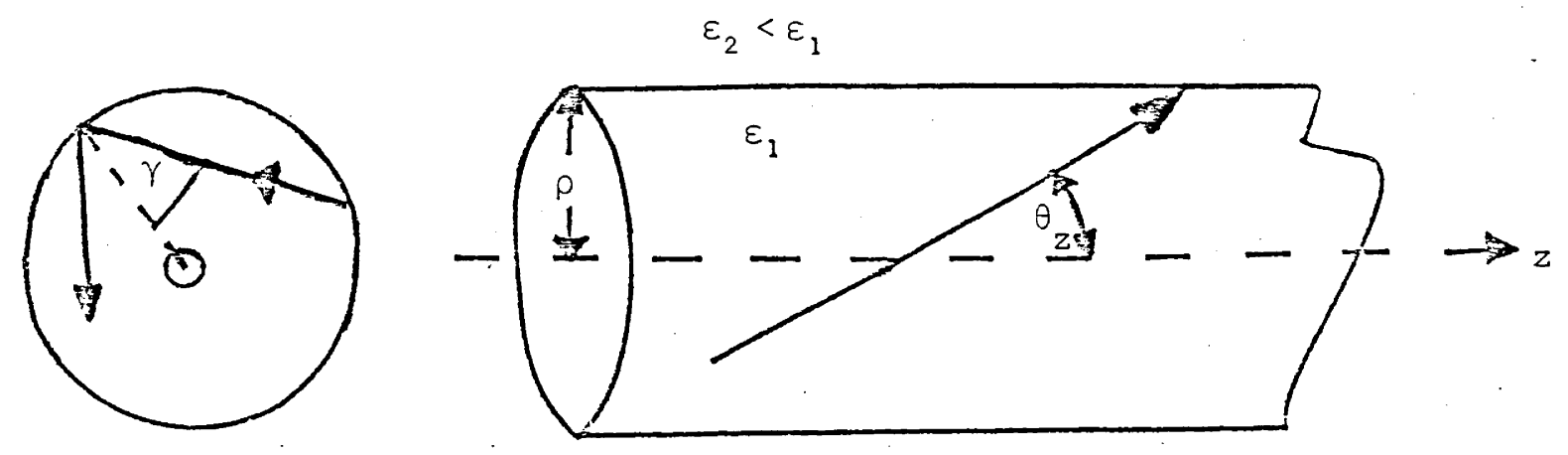

Fig. 3: The characteristic ray directions in the step-index optical fibre $\theta_{z}$ is the angle to the $z$ direction and $\gamma$ is the angle between the radial direction and the ray direction in the cross section of the fibre at the core-cladding interface. 
Current Method has increased accuracy for scattering centres located away from the waveguide axis. In the Volume Current Method all radiation, whether it is coupled into strongly refracting rays or weakly leaky rays is assumed to leave the waveguide immediately and play no further role in the propagation of a pulse along the waveguide. This is an obvious flaw in the technique and, as will be discussed in Chapter 4, this should be corrected. Ignoring any coupling of the radiation scattered into the leaky rays back into the bound rays of the fibre, we observe then that eventually all this power will be lost from the waveguide so that in this respect the Volume Current Method would provide an accurate assessment of the power lost. However, it cannot provide an accurate radiation pattern for irregularities in dielectric waveguides since the method cannot account for only the partial refraction of the radiation at each incidence on the core-cladding boundary.

Using the dyadic Green's function of the unbounded dielectric medium, $\varepsilon_{2}$ and $\mu_{2}$, as an approximation to the exact dyadic Green's function for the weakly guiding fibre in equations (11) and (12), and taking the far-zone expression for this function [18], the electromagnetic fields in the radiation zone as $R \rightarrow \infty$ (i.e. far from the core of the fibre), viz. $\underset{\mathrm{S}_{\mathrm{S}}^{\mathrm{R}}}{\mathrm{H}_{\mathrm{H}}^{\mathrm{R}}} \mathrm{\sim}^{\prime}$, can be written as

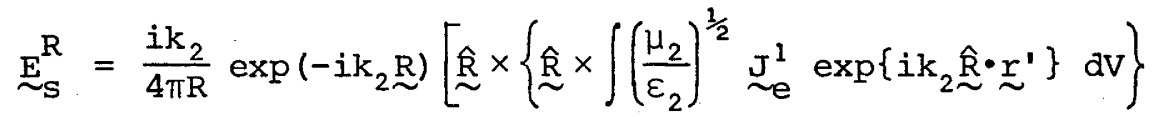

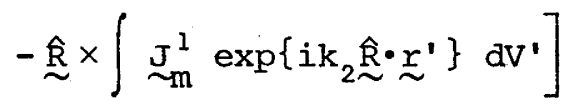

and

$$
\underset{\mathrm{S}}{\mathrm{H}^{\mathrm{R}}}=\left(\frac{\varepsilon_{2}}{\mu}\right)^{\frac{1}{2}}\left(\underset{\sim}{\hat{\mathrm{R}}} \times \underset{\mathrm{E}^{\mathrm{R}}}{\sim}\right)
$$

as $R \rightarrow \infty$. The extra superscript $R$ indicates only that part of the scattered field that exists at infinity.

The position vector, $\stackrel{r}{\sim}^{\prime}$, refers to the location of the source and $\underset{\sim}{\mathrm{R}}$ is the unit radial vector relative to the origin located on the fibre axis.

From equations (14) and (15) the total power radiated, $\mathrm{P}^{\mathrm{R}}$, by the imperfections is then given by [13] 


$$
P^{R}=R^{2} \int^{2 \pi} d \phi \int^{\pi} S^{R} \sin \theta d \theta,
$$

where $\mathrm{S}^{\mathrm{R}}$ is the radial component of the time averaged Poynting vector defined by

$$
S^{R}=\frac{1}{2}{\underset{\sim}{E}}_{\sim}^{R} \times \underset{\sim}{H^{R *}} \cdot \underset{\sim}{\hat{R}}
$$

which from equation (15) becomes

$$
S^{R}=\frac{1}{2}\left(\frac{\varepsilon_{2}}{\mu}\right)^{\frac{1}{2}}\left|\stackrel{\mathrm{E}}{\mathrm{R}}^{2}\right|^{2} .
$$

Therefore, if we know the distribution of electric and magnetic current densities in the dielectric waveguide, from equations (14), (16) and (18), the total power radiated can be determined.

For the dielectric waveguide with imperfections, the form of the pseudo electric and magnetic current densities vi.a equations (7) and (8) is unknown, since in general, the field at the location of the irregularity is unknown. However, for very slight imperfections, the incident field at the location of the irregularity can be approximated by the field at this location in the ideal, unperturbed guide. If we now restrict the discussion to dielectric irregularities, using this assumption the radiation fields can be readily determined by direct integration of equation (14) with

$$
\widetilde{J}_{e}(\underline{r})=i \omega(\Delta \varepsilon) \underset{\sim}{E_{0}}(\underline{r}),
$$

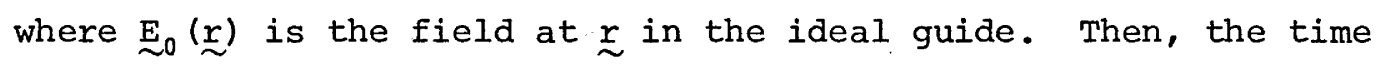
average Poynting vector defined in equation (17) becomes

$$
S^{R}=C\left|\underset{\sim}{\hat{R}} \times \int \underset{\sim}{J_{e}^{1}} \exp \left\{i k_{2} \underset{\sim}{R} \cdot \underset{\sim}{\underline{r}}{ }^{\prime}\right\} d V^{\prime}\right|^{2},
$$

where $\mathrm{J}^{1}$ is defined in equation (19) and the constant $\mathrm{C}$ is

$$
C=\left(\frac{\mu}{\varepsilon_{2}}\right)^{\frac{1}{2}} \frac{k_{2}^{2}}{32(\pi R)^{2}} .
$$

We have now reduced the problem of determining the radiated 
power from dielectric irregularities to a very simple antenna calculation, depending on the incident unperturbed field. In the last two sections this unperturbed field will be taken to be one of the bound modes of the waveguide.

The approximations involved in arriving at the equations of the Volume Current Method, i.e. equations (19) - (21), are such that the method is valid for a general class of dielectric waveguides. (This is not meant to imply that it is equally accurate for all such waveguides in which the dielectric difference between the core and cladding is the same. Once again this question must remain unanswered until knowledge of the form of the dyadic Green's function for each structure is known in a form amenable to calculations.) Only the amplitude of the incident electric field, equation (19), carries any information as to the dielectric profile under consideration, when the volume Current Method is used.

In general, power propagating in an optical waveguide is distributed among many bound modes. As the magnitude of the radiation loss induced by a dielectric irregularity is generally mode dependent, the effect of the irregularity on the total power propagating in a multimode waveguide is a complex problem. Snyder, in his review [19], discusses situations for which the complexity is minimal. These situations fall into two categories, viz.

(1) Irregularities randomly displaced with no correlation between their positions: In this situation the power from each irregularity can be added without considering interference.

(2) Multimode waveguides, with equal excitation of many modes: The field within such a guide has a high degree of incoherence (by virtue of the equal excitation of the modes). This case is treated analogously to illumination by incoherent sources [19].

The reader is referred to ref. 5 in which Marcuse discusses the transition situations which possess a degree of correlation between scatterers. In the practical situation, the effect of correlations between irregularities can only be introduced in an empirical fashion by 
determination of the correlation function that provides the best fit to the data.

\subsection{THE EQUIVALENCE OF THE VOLUME CURRENT METHOD AND APPROXIMATE COUPLED MODE THEORY}

of the many theoretical techniques that have been used to study the losses associated with irregularities in dielectric waveguides, the two most applicable techniques are Coupled Mode Theory and the Volume Current Method. The applicability of Coupled Mode Theory relies upon knowledge of the radiation modes of the waveguide, which are difficult to calculate and apply, due to difficulty of normalisation of these modes. Therefore, any approximate technique has great appeal and a thorough investigation of its validity and relationship with the exact Coupled Mode Theory should be made. For the weakly guiding fibre, the Volume Current Method, developed in the previous section, is such a technique. Another approach frequently used is the simplification of Coupled Mode Theory by approximating the exact radiation modes by the free space radiation modes $[5,20]$. Although this latter technique reduces the level of complexity of Coupled Mode treatments, it does not eliminate the problem of normalisation of the radiation modes.

In facility of calculation, the Volume current Method is superior to the Coupled Mode treatments (including the approximate Coupled Mode Theory discussed above). Until recently, no theoretical studies existed that related the two techniques.

Recently Marcuse [20] in a study of the radiative losses induced by a sinusoidally perturbed core-cladding interface of a stepindex fibre, demonstrated the equivalence of the results obtained by the Volume Current Method and the Coupled Mode Theory when the radiation modes of the fibre were approximated by the free-space radiation modes. Although this study indicated that the equivalence was more general, it did not provide a rigorous proof.

More recently, white and Snyder [21] proved that this equivalence was completely general and extended to the case of graded 
index fibres under the same approximations in Coupled Mode Theory. Such a proof is desirable as the more involved calculations via Coupled Mode Theory can be supplanted by the simpler analysis of the Volume Current Method when such approximate modes are used.

In this section, we will derive this proof for weakly guiding dielectric waveguides with an arbitrary dielectric profile. Although this proof answers the question of the relationship between the two techniques, it does not directly specify the accuracy of the Volume Current Method. In the final section of this chapter we will go some of the way to answering this question.

Let us now examine the formulae for the total power radiated from a weakly guiding optical fibre calculated by the volume Current Method and the Coupled Mode treatment, for which the radiation modes are approximated by the free space radiation modes of the cladding medium, $\varepsilon_{2}$.

For slight imperfections, the power coupled into a radiation mode with electric field, $\underset{\sim}{E_{r}}$, from an incident bound mode field, $\stackrel{\mathrm{E}}{\sim}^{\prime}$, by Coupled Mode Theory [22], using the ideal mode formulation, is

$$
S_{R}=\frac{\omega^{2}}{16}\left|\int_{V^{\prime}} d V^{\prime}\left\{\Delta \varepsilon \underset{\sim}{E_{r}^{*}} \cdot \underset{\sim}{E}\right\}\right|^{2}
$$

where $\Delta \varepsilon$ is defined in equation (1.9) and the integration is over the volume containing the irregularities, $\mathrm{V}^{\prime}$.

The free space radiation modes of the cladding media are [23]

$$
\begin{aligned}
& \underset{r}{E_{r}^{j}}={\underset{\sim}{a}}_{j}\left[\frac{1}{2 \pi}\left\{\frac{2\left(\frac{\mu}{\varepsilon_{2}}\right)^{\frac{1}{2}} k_{2}}{\beta}\right\}^{\frac{1}{2}} \exp \left\{-i k_{2} \underset{\sim}{\stackrel{R}{R}} \cdot \stackrel{r}{\prime}^{\prime}\right\}\right] \\
& \underset{\sim}{\stackrel{\mathrm{H}}{\mathrm{j}}}=\left(\frac{\varepsilon_{2}}{\mu}\right)^{\frac{1}{2}} \stackrel{\underset{\sim}{\mathrm{R}}}{\underset{\sim}{\mathrm{E}_{r}^{j}}},
\end{aligned}
$$

where the direction of propagation of the radiation field is $\underset{\sim}{\mathbb{R}}$. The superscript, $j(=1,2)$, refers to the two independent polarisation states of the radiation fields, such that the polarisation vectors, ${\underset{j}{j}}^{\prime}$ 
satisfy

and

$$
\left.\begin{array}{l}
\hat{a}_{1} \times \hat{a}_{2}=\hat{R} \\
\hat{a}_{1} \cdot \hat{a}_{2}=0
\end{array}\right\} .
$$

$\mathrm{k}_{2}$ is the wavenumber of the radiation and is the magnitude of the wavevector, $\mathrm{k}_{2}$, defined by

$$
\underline{k}_{2}=k_{2} R=\kappa_{r} \underset{\sim}{\hat{x}}+\gamma_{r} \hat{z}+\beta_{r} \hat{z},
$$

where $\beta_{r}$ is the axial propagation constant.

The total power radiated, $\mathrm{P}_{\mathrm{R}^{\prime}}$ from the fibre is obtained by summing over all the radiation modes, which yields

$$
P_{R}=\int_{j} S_{R} d k_{r} d \gamma_{r},
$$

where the $\Sigma_{j}$ indicates a summation over the polarisation states.

By converting equation (5) to an integration over the solid angle with the z-asis of the fibre parallel to the z-axis of the spherical co-ordinate system of the solid angle, $P_{R}$ becomes [24]

$$
P_{R}=\sum_{j} \int\left(S_{R} k_{2} \beta_{r}\right) d \Omega .
$$

Then, using equations (1) - (4) in equations (5) and (6), the expression for the total power radiated becomes

$$
\mathrm{P}_{\mathrm{R}}=\left(\frac{\mu}{\varepsilon_{2}}\right)^{\frac{1}{2}} \frac{\omega^{2} \mathrm{k}_{2}^{2}}{32(\pi)^{2}} \int \mathrm{d} \Omega\left\{\left|\xi_{\nu 1}\right|^{2}+\left|\xi_{\nu 2}\right|^{2}\right\},
$$

where

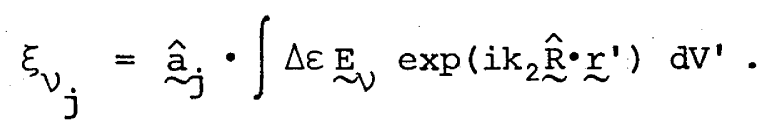

Thus, equations (7) and (8) provide the expression for the power radiated from dielectric irregularities calculated by coupled Mode Theory.

From the equations of the Volume Current Method, equations $(1.19)-(1.21)$, the total power radiated, $\mathrm{P}_{\mathrm{R}^{\prime}}^{\prime}$ is 


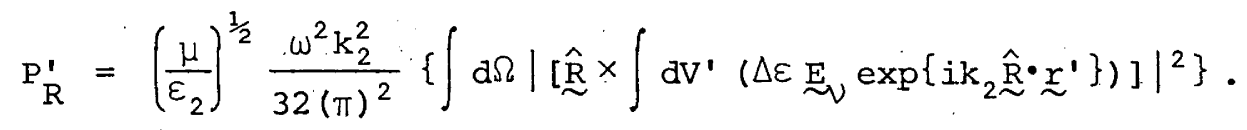

On using equation (4) with the definition, equation (8), this result becomes

$$
P_{R}^{\prime}=\left(\frac{\mu}{\varepsilon_{2}}\right)^{\frac{1}{2}} \frac{\omega^{2} k_{2}^{2}}{32(\pi)^{2}}\left[\int d \dot { \Omega } \left\{\left|\xi_{\nu I}\right|^{2}+\left|\xi_{\nu 2}\right|^{2}\{]\right.\right.
$$

which from comparison with equation (7) reveals the equivalence of the two calculations.

We have shown that when the Coupled Mode Theory is used with the radiation modes of the fibre approximated by the free space radiation modes of the cladding, the calculation is equivalent to that of the Volume Current Method. This proof demonstrates clearly the inherent redundancy in the formalistic treatment of radiative losses by Coupled Mode Theory, when used with such approximations. The Coupled Mode treatment requires explicit incorporation of both independent polarisation states of the radiation field, whereas the Volume Current Method automatically includes both polarisations.

For irregularities whose dielectric permittivity is much greater than the waveguide permittivity, the exact Coupled Mode Theory treatment must be used, since the fields in the vicinity of the irregularity can no longer be well approximated by the incident electromagnetic field. However, the Coupled Mode Theory treatment is not uniformly valid for all irregularities, and there is much debate in the literature on the applicability of such an analysis when the imperfections are not slight [25].

\subsection{APPLICATIONS OF THE VOLUME CURRENT METHOD}

In the preceding sections we discussed the philosophy of the Volume Current Method and considered its relationship with the classical treatment by Coupled Mode Theory for the determination of radiative loss from slight dielectric irregularities. Having established this formulation, we next present two examples of radiation mechanisms of 
practical importance, that demonstrate the facility of the Volume Current Method. This analysis will employ the general modal field formulation established in Chapter 2 and will be restricted to examining the losses associated with individual modes of the optical fibre.

The two most commonly observed dielectric irregularities are isolated irregularities in the core and variations in the structural geometry of the waveguide. These irregularities are induced during the manufacture of the optical fibre. In the following sections we will determine the modal power losses due to these radiation mechanisms for modes incident with unit power. In the discussion, we shall present quantitative results for the step-index fibre in order to demonstrate the accuracy of the volume current Method.

\subsection{1(a) Radiation Loss from Isolated Irregularities}

When an electromagnetic wave is incident on a dielectric irregularity, a portion of the incident energy is scattered and redistributed among the bound modes and radiation modes of the waveguide. We will only concern ourselves with that portion redistributed into the radiation field as this is the component of most significance in determination of the attenuation characteristics of the fibre. The "ultimate" transparent material is limited by the molecular granularity of the material. Such intrinsic fluctuations in the dielectric permittivity must therefore give rise to radiative loss from any dielectric structure. In this section we shall determine the effect of such irregularities since they will impose the fundamental limitation on the attenuation characteristics of optical fibres.

The deliberate introduction of dielectric irregularities into the dielectric optical fibre has been postulated as a technique to improve the impulse response of multimode waveguides by inducing deliberate mode-mixing [26]. This technique, however, proves to be of little significance for step-index fibres as the associated loss penalty on the total power propagating in the pulse is too large for the small gain in impulse response. 
In practice, isolated dielectric irregularities appear most frequently at the core-cladding interface due to small foreign particles adhering to the interior of the glass tubing that ultimately forms the inner cladding of the fibre. However, we shall not restrict the location of the scatterers except that they are in the core of the fibre. Due to the rapidly decaying evanescent field in the cladding, scattering centres beyond the core have little effect on the bound modes of the fibre.

As in fig. 3, we consider a small dielectric irregularity of volume $V$, and dielectric permittivity $\varepsilon_{3}^{\prime}\left(\sim \varepsilon_{1}\right)$ located at $\underset{\sim}{r}=\underline{x}_{0}$ in the core of the waveguide. If the dimensions of the irregularity are much less than the wavelength of the incident field, the electric field can be assumed constant over the irregularity and the power radiated from an incident mode $\mathrm{E}_{\ell \mathrm{m}}$ (of the unperturbed fibre) due to this scattering centre is (see Appendix B)

$$
P^{R}=R^{2} \int_{0}^{2 \pi} d \phi \int_{0}^{\pi} \sin \theta d \theta s^{R},
$$

where $S^{R}$ is the time averaged Poynting vector

$$
S^{R}=(V \omega \Delta \varepsilon)^{2} \quad C\left|\underset{\sim}{\stackrel{R}{R}} \times{\underset{\sim}{l m}}_{l}\left(\underline{r}_{0}\right)\right|^{2},
$$

where $C$ is defined by equation (1.21) and $\Delta \varepsilon=\left(\varepsilon_{1}-\varepsilon_{3}^{\prime}\right)$.

The modal field, from equations (2.2.42)-(2.2.50) written in cartesian co-ordinates is

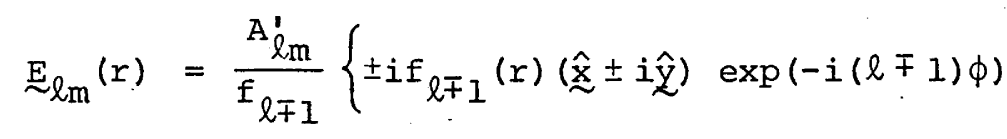

$$
\begin{aligned}
& \left.-\frac{1}{N_{\ell \mp 1}} f_{\ell}(r) \exp (-i \ell \phi) \underset{\sim}{\hat{z}}\right\},
\end{aligned}
$$

where $\underset{\sim}{\hat{z}}$ is parallel to the axis of the fibre.

In practical weakly guiding fibres, the longitudinal components of the field are of order $\theta_{C}$ compared to the transverse fields and are usually ignored. However, we have retained this 


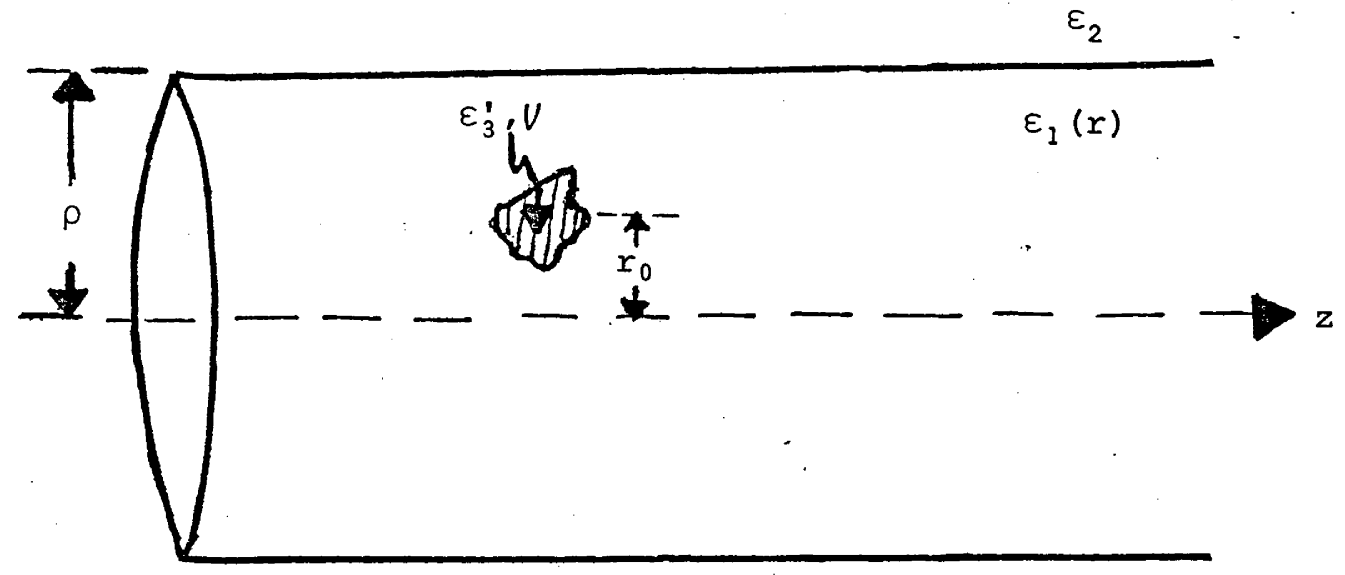

Fig. 3: An isolated dielectric irregularity of dielectric permittivity $\varepsilon_{3}^{\prime}$, and volume $V$ located a distance $r_{0}$ from the fibre axis. 
component to this point to determine the effect of a scalar located on the axis of the waveguide. From the power series expansion for $f_{p}(r)$ around $r=0$, it can be shown that only the functions $f_{0}(r)$ are non-zero at $r=0$.

Thus, irregularities on the axis of the fibre scatter power from the $\mathrm{HE}_{1 \mathrm{~m}}$ and $\mathrm{TM}_{\mathrm{Om}}$ modes only. ${ }^{+}$The $\mathrm{HE}_{1 \mathrm{~m}}$ modes have non-zero transverse electric fields on the fibre axis, whereas from equations $(2.2 .43)-(2.2 .48)$ the $\mathrm{TM}_{\text {Om }}$ modes have non-zero longitudinal electric fields on axis. In weakly guiding fibres, the transverse fields are much larger than the longitudinal fields and we would therefore expect the $\mathrm{HE}_{1 \mathrm{~m}}$ modes to have a much greater radiation loss than the $\mathrm{TM}_{\mathrm{Om}}$ modes for scatterers located on the fibre axis. Yip et al. [7] have studied the effect of scatterers located on axis but do not discuss the scattering from the $\mathrm{TM}_{\mathrm{Om}}$ modes although such an effect is easily included in their dyadic Green's function analysis.

In general, the magnitude of the longitudinal fields can be ignored in calculations of the total power radiated. (This is not true, however, of the angular spectrum of the radiation since the longitudinal fields contribute significantly to the power radiated in the transverse direction, however small in comparison to the power flowing in other directions. We will discuss this more fully at a later stage.)

In the practical situation, the interest is centred on the total power radiated and thus, ignoring the longitudinal fields, the total power radiated from the $l \mathrm{~m}^{\text {th }}$ mode, $\mathrm{P}^{\mathrm{R}}$, is (see Appendix $\mathrm{B}$ )

$$
P^{R}\left(U_{l m}\right)=\frac{1}{6 \pi}\left(\frac{\mu}{\varepsilon_{2}}\right)^{\frac{1}{2}}(\omega V \Delta \varepsilon)^{2} \cdot k_{2}^{2}\left|\frac{A_{l \mp 1} f_{l \mp 1}(r)}{f_{l \mp 1}(\rho)}\right|^{2} .
$$

If we now consider the particular case of an irregularity located at the core-cladding boundary interface of a step-index fibre, then using equations (2.3.1) - (2.3.11) for the modes of the step-index fibre, we have

\footnotetext{
+ Since the $\mathrm{TE}_{\mathrm{Om}}$ modes have no electric field on the fibre axis they cannot scatter any energy due to axially located irregularities.
} 


$$
P^{R}\left(U_{l m}\right)=\frac{1}{6 \pi^{2}}\left(\frac{(\Delta \varepsilon)}{\varepsilon_{1}} \frac{V}{\rho}\right)^{2}\left(\frac{k_{1}^{2} U}{V^{2}}\right)^{2} \xi_{l m}
$$

where

$$
\xi_{\ell m}=\frac{\mathrm{H}_{\ell \mp I}^{(1)}}{\mathrm{H}_{\ell \mp 2}^{(1)} \cdot \mathrm{H}_{\ell}^{(1)}}
$$

with the argument of the Hankel functions as (iW) (the subscript, lm, is omitted from the equation for conciseness).

This result is in agreement with snyder's calculation except for an error of a factor of $(1 / \pi)$ in Snyder's result [19]. Except close to the mode cutoff the term $\xi_{l m}$ defined in equation (6) is approximately unity [19]. Using the asymptotic expressions for this term and for $\mathrm{U}_{\ell \mathrm{m}}(\mathrm{V})$ one can obtain useful practical formulae for modal power losses as a function of $V$, the waveguide parameter [19], where $V$ is defined to be

$$
\begin{aligned}
V & =k_{1} \rho\left(1-\frac{\varepsilon_{2}}{\varepsilon_{1}}\right)^{\frac{1}{2}} \\
& =k_{1} \rho \theta_{c} .
\end{aligned}
$$

From equation (5), the higher order modes, i.e. $U_{l m} \rightarrow V$, radiate more than the lower order modes. This is because the intensity of the fields of the higher order modes is much larger at the corecladding interface, and hence they scatter more power.

When $\mathrm{V}>>1$, a mode can be considered as a family of rays with characteristic angle $\theta_{\ell m}$ to the waveguide axis. Then

and

$$
\beta_{\ell m}=k_{1} \cos \theta_{\ell m}
$$

$$
\mathrm{U}_{\ell \mathrm{m}}=\mathrm{k}_{\mathrm{l}} \rho \sin \theta_{\ell \mathrm{m}}
$$

Using equations (7), (8) in equation (5) we find

$$
\mathrm{P}^{\mathrm{R}}\left(\theta_{\ell \mathrm{m}}\right)=\left(\frac{1}{6 \pi^{2}}\right)\left(\frac{V}{\rho}\right)^{2}\left(1-\frac{\varepsilon_{3}}{\varepsilon_{1}}\right)^{2}\left(\frac{\mathrm{k}_{1}^{2} \theta_{\ell m}}{\theta_{\mathrm{c}}}\right)^{2}
$$

where we have explicitly written $\Delta \varepsilon=\left(1-\varepsilon_{3} / \varepsilon_{1}\right)$, where $\varepsilon_{3}$ is the 
dielectric permittivity of the imperfection. We have taken the far from cutoff expression for $\xi_{l_{m}}(=1)$ in equation (9) [19].

In this form we can readily observe that the total power radiated obeys the $\left(\theta_{\mathrm{lm}}^{2} / \lambda^{4}\right)$. law which is a modified Rayleigh scattering law. Since the leaky rays of the fibre have angles $\theta_{l m}^{L . R}$. such that

$$
\frac{\theta_{l_{m}^{L} \cdot R .}^{L}}{\theta_{c}}>1,
$$

whereas the bound rays have angles $\theta_{z}$ such that

$$
\frac{\theta_{\text {lm }}^{\text {B. R. }}}{\theta_{C}}<1
$$

equation (9) indicates that the leaky rays of a fibre are more strongly affected by the presence of the scattering centres at the core-cladding interface.

Our analysis of the radiation from a dielectric irregularity was based upon the fields of the two region fibre. We may expect that the accuracy of this analysis is suspect for multiply clad waveguides especially for the case of the outer cladding being air with a dielectric permittivity greatly different from the core and cladding dielectrics. This large change in dielectric permittivity will change the character of a large fraction of the radiation from free radiation that would rapidly leave the waveguide structure to power propagating in the cladding modes [10]. Yip et al. [7] have studied this problem and have published some rather surprising results.

The analysis of ref. 7 provides a comparison between the Volume Current Method (called the infinite medium approximation in the reference) and the exact analysis by a dyadic Green's function formulation for the particular case of scatterers on axis. Fig. 1 of ref. 7 provides two significant pieces of information:

(1) The extent of the inner cladding does not significantly affect the magnitude of the power radiated away from the fibre; and more surprisingly 
(2) The total power radiated from an irregularity in the dielectric waveguide is greater than if the irregularity was in free space with the same incident field.

The former result, (1) above, is obvious from a geometric optics approach (see fig. 4a). One can see that the distance that the outer-cladding interface is from the inner-cladding core interface is immaterial in determining the amount of power radiated from the structure. The dominant factor in determining the amount of radiated power is the complement, $\theta_{C^{\prime}}^{0}$ of the critical angle of the outercladding interface, $\alpha_{c}^{0}$. If $\alpha_{c}^{0}$ is large then most of the radiation from the irregularity in the core becomes power propagating in the cladding modes of the multiply clad waveguide.

Although, from the above argument, the total radiated power from the multiply clad optical fibre will not be affected by the distance between the inner and outer core-cladding interfaces, the rate at which this radiation escapes from the multiply clad fibre will vary. Since the radiation emanating from the inner-cladding boundary at an inclination $\theta_{z}>\pi / 2-\alpha_{c}^{0}$ to the axis, where $\alpha_{c}^{0}$ is, as above, the local critical angle at the outer core-cladding boundary, becomes a tunnelling leaky ray relative to the outer-cladding interface, depending on the radius of the outer-cladding surface, this radiation will escape at a rate consistent with the tunnelling coefficient at the outer boundary. As can be seen from fig. 4b, the skewness angle $\gamma^{\prime}$ which critically affects the tunnelling leaky ray loss, decreases as the radius of the outercladding interface increases relative to the inner-cladding. As the tunnelling leakage increases, with decreasing skewness of the radiation [19], the rate of loss of the radiation from the multiply clad fibre should increase as the radius of the outer-cladding is increased, i.e. in the limit of an infinite inner-cladding, the singly clad fibre result should apply.

This geometric optics argument raises doubt as to the correctness then of the calculation of Yip et al., showing that the finitely cladded fibre radiates more from an irregularity than the irregularity would, if it were in free space. Snyder states that these studies on multiply clad waveguides confirm the validity of the Volume current 


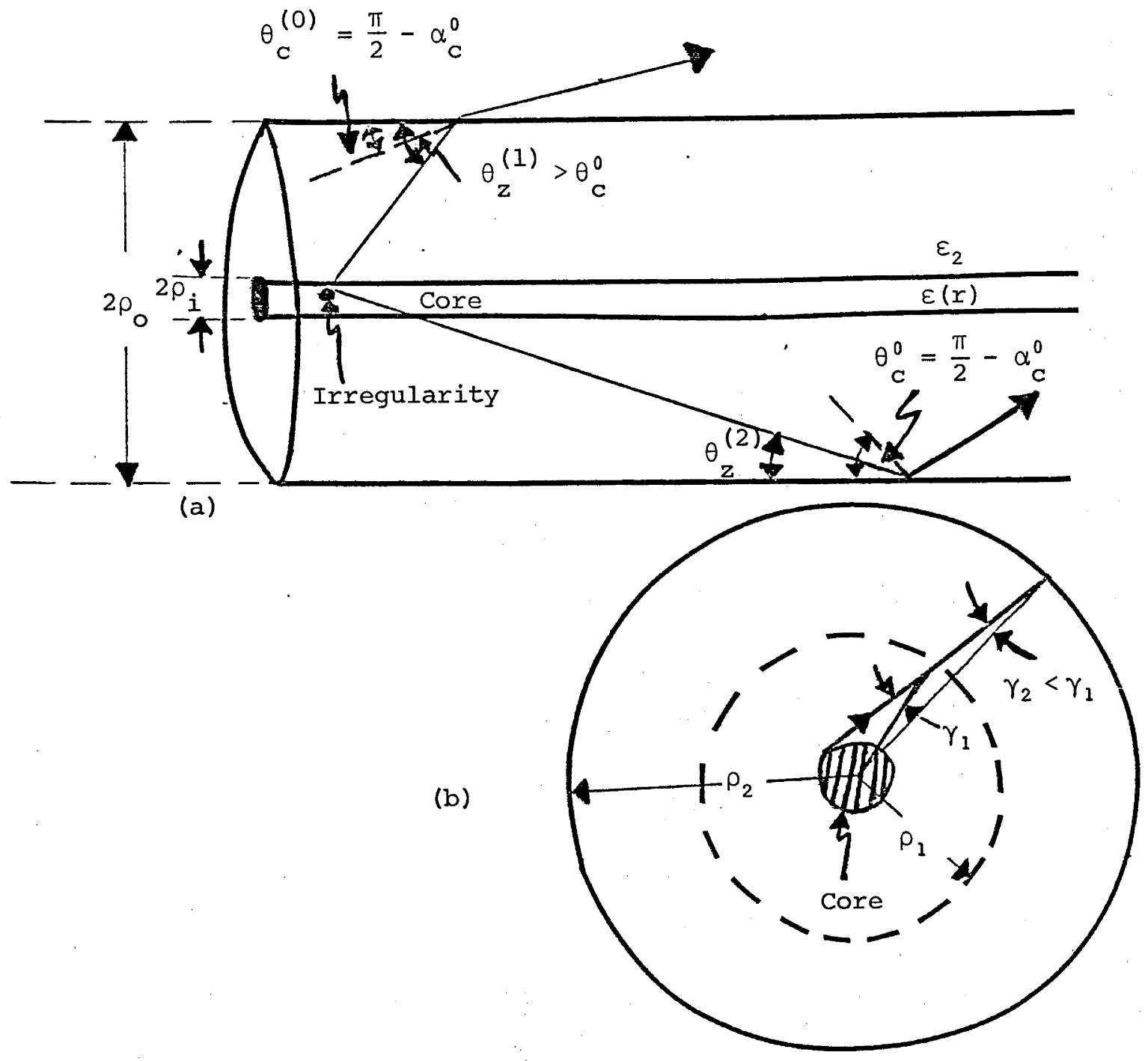

Fig. 4: Radiation from a dielectric irregularity in the presence of an outer core-cladding interface. In fig. $4 \mathrm{~b}$, we have plotted two radii for the outer core-cladding boundary, $\rho_{1}, \rho_{2}$. 
Method even down to any small outer-cladding radii of the order $\rho_{0} / \rho_{i} \sim 5$, where $\rho_{i}$ is the radius of core of the waveguide and $\rho_{0}$ is the radius of the inner cladding, as in fig. 4a. However, the author does not agree, since from the above argument, it does not appear that the ratio $\rho_{o} / \rho_{i}$ plays the dominant rôle in determining the accuracy of the Volume Current Method, except for $\rho_{0} / \rho_{i} \sim 1$, i.e. there is no innercladding. The significant factor in determining the accuracy of the Volume Current Method is the outer-cladding dielectric permittivity relative to that of the inner-cladding. Assuming that the outer-cladding is air, this factor becomes $\left(\varepsilon_{2}-\varepsilon_{0}\right)$, which is no longer very small since for typical waveguides $\varepsilon_{2} \sim 2.25 \varepsilon_{0}$ [7].

In the waveguide with infinite cladding, the core-cladding dielectric difference strongly affects only the power propagating at angles to the axis $\theta_{z} \leqslant \theta_{c}$. Thus for diffuse (i.e. wide angle) scattering processes it can be seen that the Volume Current Method should provide valid estimates of the total power radiated. The calculations are in error by including the power flowing in the $2 \pi \theta_{\mathrm{C}}^{2}$ $(\ll 1)$ steradians centred about $\theta=0$ that constitutes the power scattered into the bound modes (or rays) of the fibre. However, although all the power scattered into the directions with $\theta_{\mathbf{z}}>\theta_{\mathbf{C}}$ eventually escapes from the core of the fibre, the rate at which this power escapes depends on the nature of the ray (i.e. either a leaky tunnelling ray or a refracting ray). This effect introduces additional phase interference between the radiation fields and thus the radiation. pattern of the scattered power will not be accurately determined by the Volume Current Method, as Yip and Martucci have recently shown [8].

Let us digress, momentarily, and investigate the efficiency of the isolated point dipoles as an excitation mechanism for a monomode weakly guiding step-index waveguide. This simple study provides valuable insight into the relative accuracy of the above calculations.

\subsection{1(b) The Efficiency of Dipole Sources in Excitation of the Fundamental Mode of a Step-Index Fibre}

As we have discussed, the small isolated dielectric 
irregularity behaves as a point dipole radiator. To determine the relative accuracy of the Volume Current calculation, we need to calculate the relative amount of power coupled into the bound modes of the optical fibre in comparison to the total power radiated from the source. This is known as the excitation efficiency of a source. In this short digression, we calculate the efficiency of a point dipole in excitation of the $\mathrm{HE}_{1}$ mode of a monomode step-index fibre, using the well known excitation formula of waveguide theory [19], viz.

$$
P_{q}=\frac{1}{16}\left|\int_{V}, \stackrel{E_{q}^{*}}{\sim} \underset{\sim}{J} d V\right|^{2},
$$

where $P_{q}$ is the power coupled into the $q^{\text {th }}$ mode with electric field ${\underset{q}{q}}_{q}$ by a current source, J occupying a volume $V^{\prime}$. The electric field $\underset{{ }_{q}}{E}$ is that of the mode carrying unit power so that

$$
\frac{1}{2} \int_{A_{\infty}} \underset{\sim q}{E} \times \underset{\sim}{H_{q}^{*}} \cdot \underset{\sim}{z} d A=1
$$

where $A_{\infty}$ is the infinite cross-sectional plane.

From equation (10) it is obvious that equal power is coupled into the forward (+ve $z$ ) and backward (-ve z) travelling $\mathrm{HE}_{11}$ mode for a point source. For an isolated point dipole located at a distance $r_{d}$ from the fibre axis, and angle $\phi=\phi_{d}$ in the transverse plane, and at a distance $z=z_{d}$ down the fibre, the volume current density generated by the dipole moment can be written in cylindrical co-ordinates as

$$
J_{d}=\frac{\delta\left(\phi-\phi_{d}\right) \delta\left(r-r_{d}\right) \delta\left(z-z_{d}\right)}{r_{d}} J_{d},
$$

where $J_{d}$ is the dipole current which for a harmonic time variation $\left(e^{i \omega t}\right)$ can be written in terms of the dipole moment $p_{d}$

$$
\stackrel{J}{\sim}=i \omega p_{d} .
$$

equations (1) and (2)

The total power radiated from this dipole $\mathrm{P}_{\text {rad }}$ is, using 


$$
\mathrm{P}_{\mathrm{rad}}=\left(\frac{\varepsilon_{1}}{\mu}\right)^{\frac{1}{2}} \frac{\mathrm{k}_{0}^{4}}{12 \pi} \frac{\left|\mathrm{J}_{\mathrm{d}}\right|^{2}}{\omega^{2}},
$$

where $k_{0}$ is the wavenumber of the light in free space.

Using equation (10) and the electric fields of the normalised electric field of the $\mathrm{HE}_{11}$ modes the amount of power coupled into the forwardly directed $\mathrm{HE}_{11}$ mode, by a transversely oriented point dipole, is $^{\dagger}$

$$
\mathrm{P}_{\operatorname{Tr}}=\left(\frac{\mu}{\varepsilon_{1}}\right)^{\frac{1}{2}} \cdot \frac{\left|\mathrm{J}_{\mathrm{d}}\right|^{2}}{8 \pi \rho^{2}}\left(\frac{\mathrm{U}}{\mathrm{V}}\right)^{2} \cdot\left(\frac{\mathrm{J}_{0}\left(\mathrm{U} \frac{r_{\mathrm{d}}}{\rho}\right)}{\mathrm{J}_{0}(\mathrm{U})} \cdot \frac{\mathrm{H}_{0}^{(1)}(\mathrm{iW})}{\mathrm{H}_{1}^{(1)}(\mathrm{i} W)}\right)^{2}
$$

and therefore, from equations (14) and (15), the efficiency of excitation of the fundamental mode by a transversely oriented point dipole, $\xi_{T^{\prime}}$ becomes

$$
\begin{aligned}
\xi_{\mathrm{T}} & =\frac{\mathrm{P}_{\mathrm{Tr}}}{\mathrm{P}_{\mathrm{rad}}} \\
& =\Delta \cdot\left(\frac{3}{2}\left(\frac{\mathrm{U}}{\mathrm{V}^{2}}\right)^{2}\left(\frac{\mathrm{J}_{0}\left(\mathrm{U} \frac{r_{\mathrm{d}}}{\rho}\right)}{\mathrm{J}_{0}(\mathrm{U})} \cdot \frac{\mathrm{H}_{0}^{(1)}(\mathrm{iW})}{\mathrm{H}_{1}^{(1)}(\mathrm{iW})}\right)^{2}\right)
\end{aligned}
$$

where $\Delta=\left(1-\varepsilon_{2} / \varepsilon_{1}\right)<1$ for weakly guiding fibres.

We are now in a position to examine the accuracy of our Volume Current calculations. If the amount of power re-scattered into the fundamental mode is a significant fraction of the total radiated power from the dipole, then the analysis of the volume Current Method will induce significant errors. However, in fig. 5, where $\xi_{T}$ is plotted against the radial distance from the waveguide axis, $r_{d^{\prime}}$ of the dipole, we can see that this efficiency in excitation of the $\mathrm{HE}_{11}$ mode never exceeds $2.41 \Delta<1$ for $a \mathrm{~V}=2.4$ fibre.

Thus the Volume Current Method should provide extremely

+ This result is half that reported by Clarricoats [10], as he includes the backward mode coupling to the $\mathrm{HE}_{11}$ mode travelling in the negative $z$-direction. The author considers this as a power loss from a positive $z$-directed mode, therefore we lump this power in with the radiation from the guide. Due to the smallness of the mode coupling, it does not alter the overall conclusions. 


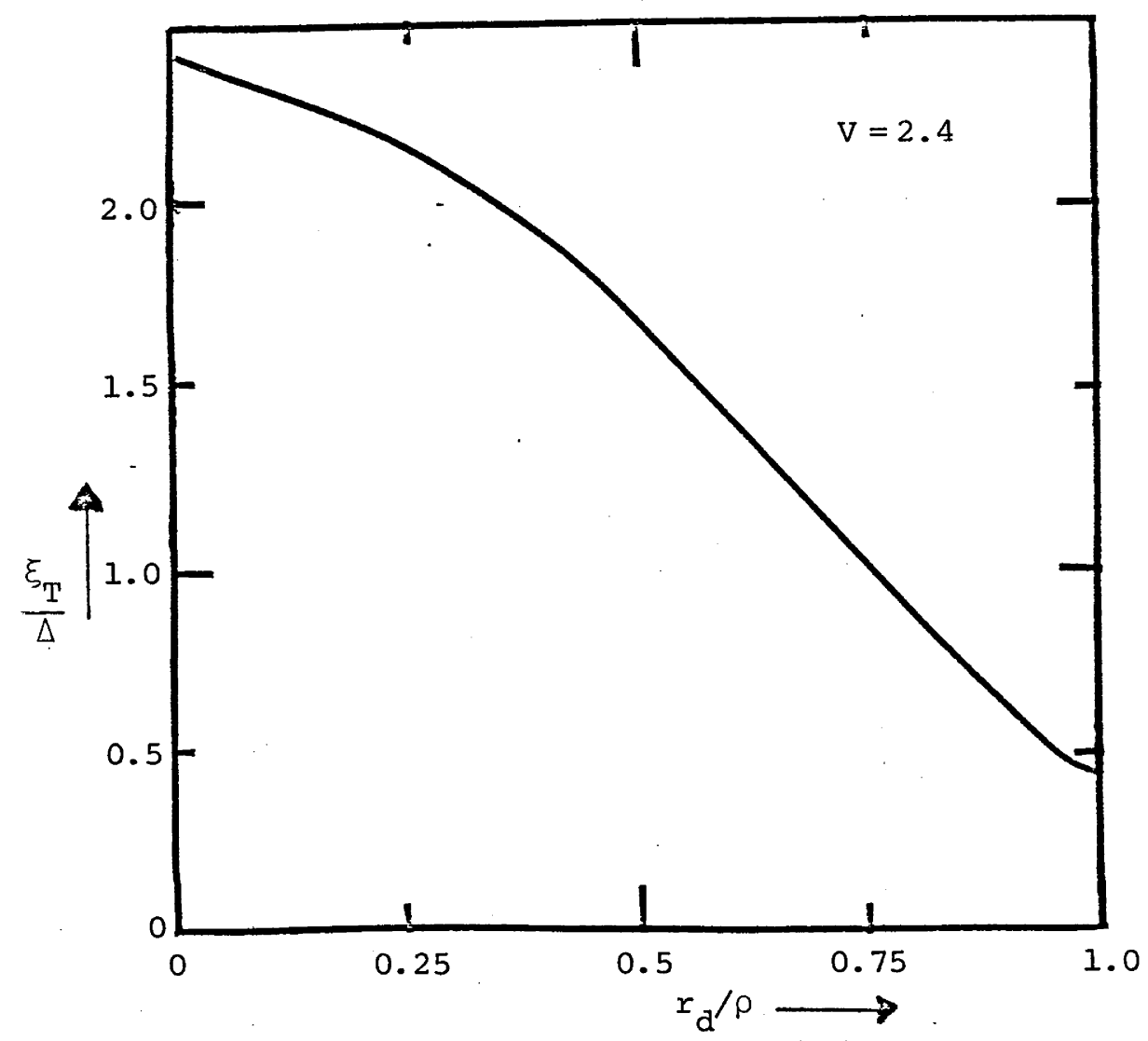

Fig. 5: The normalised efficiency of excitation, $\xi_{T} / \Delta$, of the fundamental $\left(\mathrm{HE}_{11}\right)$ mode of a step-index fibre, from equation (3.16), as a function of the distance from the waveguide axis, $r_{d}$, of the transversely oriented point dipole source, where $\Delta=\theta_{c}^{2}$. 
accurate results for weakly guiding fibres with small dielectric irregularities, as anticipated from the physical intuition. Before we return to the Volume Current analysis let us also calculate the efficiency of excitation for longitudinally oriented dipoles.

For dipoles oriented along the fibre axis, so that

$$
\underset{\mathrm{J}}{\mathrm{J}}=\mathrm{J}_{\mathrm{d}} \underset{\mathrm{z}}{\sim}
$$

the excitation occurs through the coupling to the longitudinal electric fields and again using equations (2.3.1) - (2.3.9) and equations (10) and (17), the amount of power coupled into the two forward travelling $\mathrm{HE}_{11}$ modes, due to longitudinally oriented dipoles, $\mathrm{P}_{\mathrm{z}^{\prime}}$ is

$$
\mathrm{P}_{\mathrm{z}}=\Delta\left(\frac{\mu}{\varepsilon_{1}}\right)^{\frac{1}{2}} \frac{\mathrm{J}_{\mathrm{d}}^{2}}{8 \pi \rho^{2}}\left(\frac{\mathrm{U}^{2}}{\mathrm{v}^{2}}\right)^{2} \cdot\left(\frac{\mathrm{J}_{1}\left(\mathrm{U} \frac{r_{\mathrm{d}}}{\rho}\right)}{\mathrm{J}_{0}(\mathrm{U})} \cdot \frac{\mathrm{H}_{0}^{(1)}(\mathrm{iW})}{\mathrm{H}_{1}^{(1)}(\mathrm{iW})}\right)^{2}
$$

and the efficiency of this excitation $\xi_{z}$ is

$$
\begin{aligned}
\xi_{z} & =\frac{P_{z}}{P_{\text {rad }}} \\
& =\Delta^{2}\left\{\frac{3}{2}\left(\frac{\mathrm{U}^{2}}{\mathrm{~V}^{3}}\right)^{2}\left(\frac{\mathrm{J}_{1}\left(\mathrm{U} \frac{r_{\mathrm{d}}}{\rho}\right)}{\mathrm{J}_{0}(\mathrm{U})} \cdot \frac{\mathrm{H}_{0}^{(1)}(i W)}{\mathrm{H}_{1}^{(1)}(\mathrm{iW})}\right)^{2}\right\} .
\end{aligned}
$$

Comparing the efficiencies of excitation for these two orientations of the dipoles, we find

$$
\frac{\xi_{\mathrm{z}}}{\xi_{\mathrm{T}}}=\Delta\left(\frac{\mathrm{U}}{\mathrm{V}}\right)^{2}\left(\frac{\mathrm{J}_{1}\left(\mathrm{U} \frac{r_{\mathrm{d}}}{\rho}\right)}{\mathrm{J}_{0}\left(\mathrm{U} \frac{r_{\mathrm{d}}}{\rho}\right)}\right)^{2} .
$$

For dipoles located on the fibre axis, $r_{d}=0$, and equation (20) becomes

$$
\frac{\xi_{z}}{\xi_{T}}=0,
$$

i.e. no power is coupled into the fundamental mode by dipoles oriented parallel to the axis and on the fibre axis. 
A plot of equation (20) is shown in fig. 6 for a $V=2.4$ fibre. It can be seen that the relative efficiency $\left(\xi_{z} / \xi_{T}\right)$ is a monotonically increasing function with $\left(r_{d} / \rho\right)$ to a maximum at the core-cladding interface of

$$
\frac{\xi_{z}}{\xi_{T}} \sim 0.88 \Delta=(0.88) \cdot \theta_{C}^{2} \ll 1 .
$$

From equation (20) we can see that the dipoles oriented along the axis are much less effective than the transversely oriented dipoles, in excitation of the fundamental mode. From a consideration of the radiation patterns of the two dipoles, as in figs. 7, we can readily see the reason. For a weakly guiding fibre the acceptance angle, $\theta_{c}$ for trapped rays, is very small, and as very little radiation from a dipole travels in the direction of the axis of orientation of the dipole, the amount of power scattered into this angle, $\theta_{C^{\prime}}$ by the longitudinally oriented dipole is much smaller than for the transversely oriented dipole, and from our previous discussion, we have found that even these transversely oriented dipoles do not efficiently excite the fundamental mode. However, we can observe from figs. 7, that the longitudinally oriented dipoles can contribute significantly to the radiation in the transverse plane of the waveguide, since some directions in this plane correspond to the orientations of the transverse dipoles.

Now that we have demonstrated the accuracy of our Volume Current Method calculations, let us return to considerations of distributions of small dielectric irregularities.

\subsection{1(c) Radiation from a Random Distribution of Dielectric Irregularities}

We have derived the radiative loss from one isolated irregularity, and we shall now proceed to extend this to a random distribution of scatterers, in step-index waveguides. If we define the average density of the small irregularities to be $N_{1}$ in the core and $\bar{\varepsilon}_{3}$ and $\bar{V}_{1}$ to be the average dielectric permittivity and volume, respectively, of such irregularities, then in an elemental length $\mathrm{dz}$ ' of 


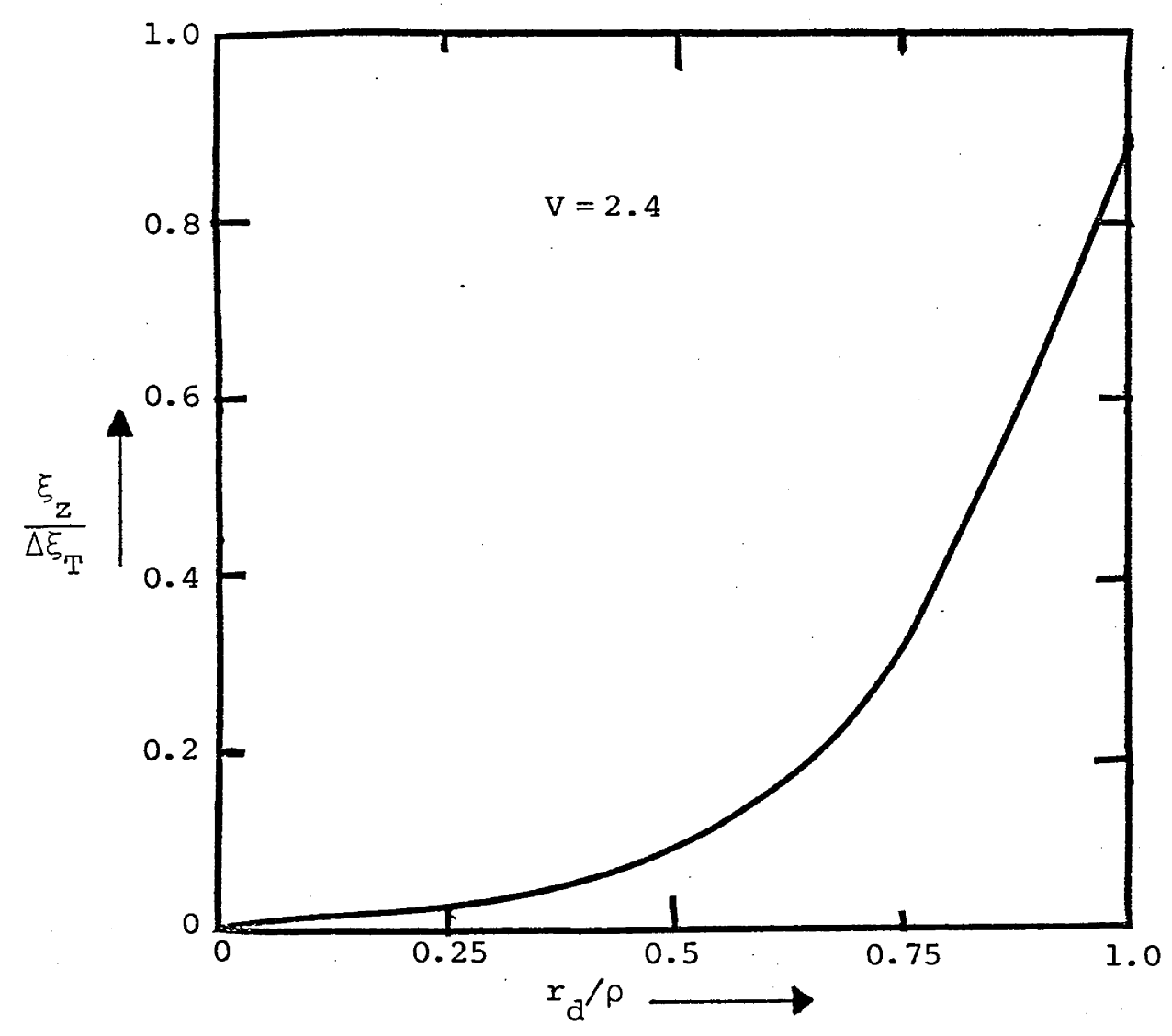

Fig. 6: The normalised ratio of the excitation efficiencies $\xi_{z} / \Delta \xi_{T}$ of the transversely and axially oriented dipoles, equation (3.20), for the excitation of the $\mathrm{HE}_{11}$ mode of a step-index fibre $\Delta=\theta_{\mathrm{C}}^{2}$. 


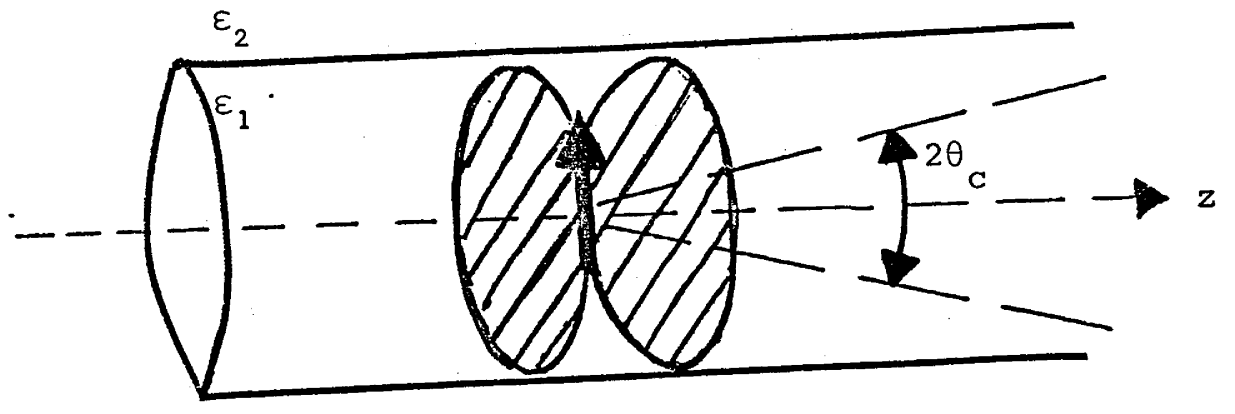

(a) Transversely Oriented Dipole

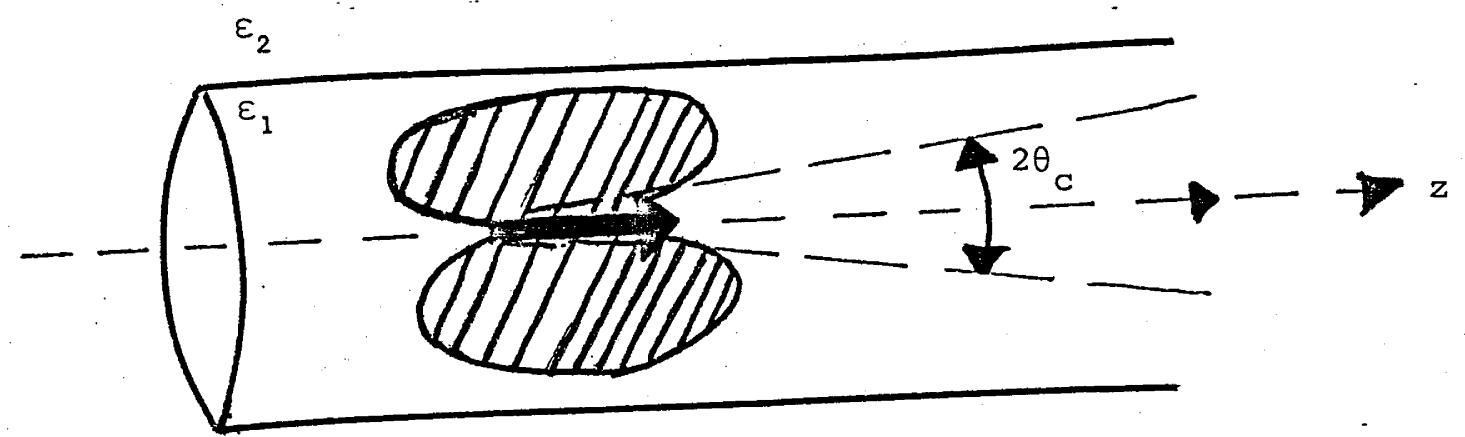

(b) Axially Oriented Dipole

Fig. 7: The radiation patterns of the transversely and axially oriented dipoles relative to the waveguide system. 
fibre, the number of scatterers in an annular cylinder, as in fig. 8, between $r$ and $r+d r$, is

$$
d N=2 \pi N_{1} r d r d z^{\prime}
$$

Since we have assumed the irregularities are randomly distributed, there is no interference between the radiation from isolated irregularities, and we therefore sum the power from each scatterer. Thus, the total power scattered from the irregularities in the hollow cylinder, using equation (5), is

$$
\mathrm{dP}_{\mathrm{R}}=\left(\frac{1}{6 \pi^{2}}\right) \cdot 2 \pi \mathrm{N}_{1}\left\{\left(1-\frac{\bar{\varepsilon}_{3}}{\varepsilon_{1}}\right) \frac{\bar{V}_{1}}{\rho^{3}}\right\}^{2}\left(\frac{\mathrm{UV}}{\theta_{\mathrm{C}}^{2}}\right)^{2} \cdot \frac{\mathrm{J}_{0}^{2}\left(\mathrm{U} \frac{r}{\rho}\right)}{J_{0}^{2}(\mathrm{U})} \cdot\left(\frac{\mathrm{H}_{0}^{(1)}(\mathrm{iW})}{\mathrm{H}_{1}^{(1)}(\mathrm{iW})}\right)^{2} r \cdot \mathrm{dr} \mathrm{dz} \mathrm{z}^{\prime}
$$

and the total radiated power from the incident $\mathrm{HE}_{11}$ mode due to a random distribution over the core is

$$
\mathrm{P}_{\text {tot }}^{\mathrm{co}}=\int_{\text {core }} \mathrm{dP}_{\mathrm{R}}
$$

and using the known integrals of Bessel functions [27], this equation becomes

$$
\mathrm{P}_{\text {tot }}^{\mathrm{co}}=\mathrm{dz} \cdot n_{1} \mathrm{~N}_{1}\left(\frac{1}{6 \pi}\right)\left\{\left(1-\frac{\bar{\varepsilon}_{3}}{\varepsilon_{1}}\right) \frac{\bar{V}_{1}}{\rho^{2}}\right\}^{2}\left(\frac{\mathrm{V}}{\theta_{c}}\right)^{4},
$$

where the superscript "co" indicates the power radiated from irregularities in the core, and $\eta_{1}$ is the fraction of the total guided power of the $\mathrm{HE}_{11}$ mode that travels within the core [19]

$$
n_{1}=\left(\frac{U}{V}\right)^{2}\left\{\left(\frac{W}{U}\right)^{2}+\left(\frac{H_{0}^{(1)}(i W)}{H_{1}^{(1)}(i W)}\right)^{2}\right\}
$$

If we consider the scatterers to be randomly distributed, throughout the cladding as well, by similar calculations to the above, we find the total power radiated in a length $\mathrm{dz}^{\prime}$, of waveguide, from irregularities in the cladding, is

$$
\mathrm{P}_{\text {tot }}^{\mathrm{Cl}}=\mathrm{dz}^{\prime}\left(1-\eta_{1}\right)\left(\frac{\varepsilon_{2}}{\varepsilon_{1}}\right)^{2}\left(\frac{1}{6 \pi}\right) \mathrm{N}_{2}\left\{\left(1-\frac{\bar{\varepsilon}_{4}}{\varepsilon_{2}}\right) \frac{\bar{V}_{2}}{\rho^{2}}\right\}^{2}\left(\frac{\mathrm{V}}{\theta_{C}}\right)^{4},
$$




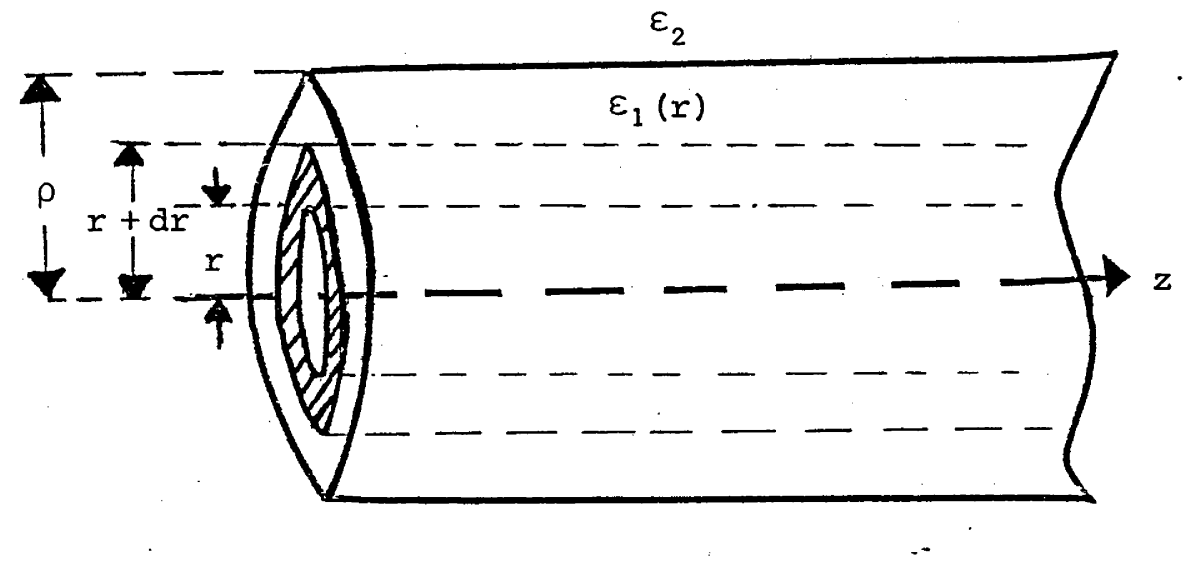

Fig. 8: An annular cylinder of radius $r$ to $r+d r$ (the shaded region) in which there exists a random collection of scattering centres, with average dielectric permittivity $\bar{\varepsilon}_{3}^{\prime}$ and volume $V_{i}^{\prime}$. 
where $\left(1-\eta_{1}\right)$ is the fraction of guided power that travels in the cladding; $\mathrm{N}_{2}, \bar{V}_{2}$ and $\bar{\varepsilon}_{4}$ have similar definitions for the dielectric irregularities in the cladding as $\mathrm{N}_{1}, \bar{V}_{1}$ and $\bar{\varepsilon}_{3}$ respectively for the irregularities of the core.

Using the equation for the normalised frequence $V$,

$$
\begin{aligned}
\mathrm{V} & =\mathrm{k}_{1} \rho \theta_{\mathrm{C}} \\
& =\frac{2 \pi}{\lambda} \mathrm{n}_{1} \theta_{\mathrm{C}^{\prime}},
\end{aligned}
$$

(where $\lambda$ is the wavelength of light in free space) we see that the power from irregularities in the core and cladding regions is proportional to $(\lambda)^{-4}$, i.e. the Rayleigh Scattering Law.

By summing the power radiated from the irregularities in the core and cladding from equations (24) and (25) respectively, the total power radiated per unit length of fibre from an $\mathrm{HE}_{11}$ mode incident with unit guided power, $\gamma$, i.e. the power attenuation coefficient becomes

$$
\gamma=\alpha_{c o} n+\alpha_{c l}(1-n)
$$

where we have defined core and cladding attenuation coefficients $\alpha_{\text {co }}{ }^{\prime} \alpha_{\text {cl }}$ respectively defined by

and

$$
\left.\begin{array}{l}
\alpha_{\mathrm{CO}}=\left(\frac{1}{6 \pi}\right) \cdot \mathrm{N}_{1}\left\{\left(1-\frac{\bar{\varepsilon}_{3}}{\varepsilon_{1}}\right) \bar{v}_{1}\right\}^{2} \mathrm{k}_{1}^{4} \\
\alpha_{\mathrm{Cl}}=\left(\frac{1}{6 \pi}\right) \cdot \mathrm{N}_{2}\left\{\left(1-\frac{\bar{\varepsilon}_{4}}{\varepsilon_{2}}\right) \bar{v}_{2}\right\} \mathrm{k}_{2}^{4} \cdot
\end{array}\right\}
$$

From a comparison of equations (28) with equation (4.7-25) of ref. 5, we observe that $\alpha_{C O}, \alpha_{C l}$ have the form of the bulk attenuation coefficients of the core and cladding materials due to randomly distributed Rayleigh scatterers.

Equation (27) represents an approximation to the general result for attenuation losses [28], which for weakly guiding optical waveguides provides a simple physical interpretation of the modal power attenuation coefficient of a straight fibre with irregularities [29], i.e. the modal power attenuation of the product of the fractional amount 
of power propagating in each region of the waveguide with the bulk absorption coefficient of that region. For an n-region fibre, this can be simply written as

$$
\gamma=\sum_{j=1}^{n} \alpha_{j} n_{j}
$$

where now we interpret $\eta_{j}$ as the fraction of power propagating in the $j^{\text {th }}$ region.

\subsubsection{Radiation Loss Due to Variations}

in the Radius of the Core

In this section we calculate the power radiated from a mode incident on a finite length of fibre with an axial variation in the radius of the core. This case is of general interest for two reasons.

(a) A statistical variation in the radius can be resolved by Fourier analysis into its sinusoidal components and each component can be studied individually before recombination of the resultant fields into the total radiation field.

(b) Machine vibrations during the manufacture and drawing of the fibre can cause small amplitude fluctuations of a constant frequency.

We shall be concerned with variations in the radius that retain the circular symmetry of the dielectric optical fibre, so that the radius of the perturbed fibre, of length $2 \mathrm{~L}$, can be written as (see fig. 9)

$$
\rho=\rho_{0}(1+a \sin \Omega z),
$$

where $\Omega$ is the spatial frequency of the perturbation and a $\rho_{0}<<$, where $\lambda$ is the wavelength of the incident field. The dielectric profile of the unperturbed fibre of radius $\rho_{0}$, as shown in fig. 10, can be described mathematically as

$$
\varepsilon(r)=\varepsilon_{1}\left(\Delta_{C l}-\Delta_{C O} q\left(\frac{r}{\rho_{0}}\right)\right) H\left(\rho_{0}-r\right)+\varepsilon_{2},
$$




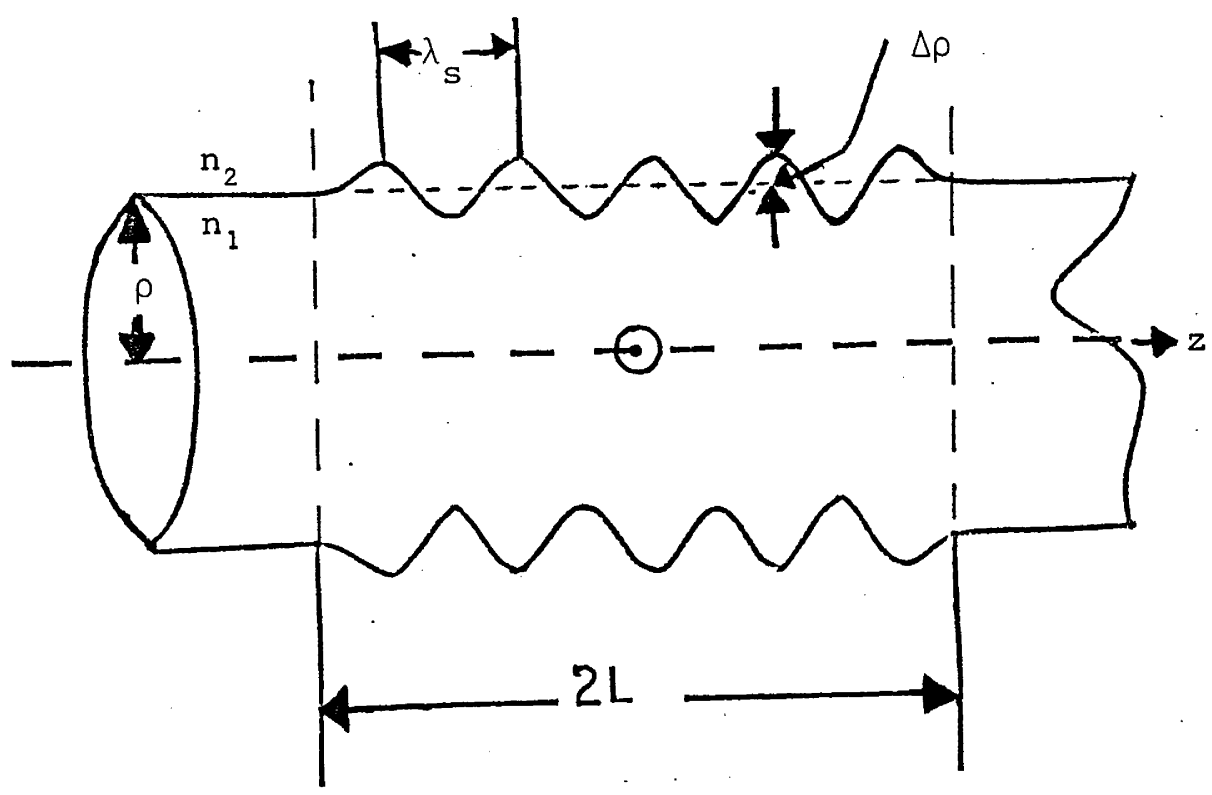

Fig. 9: Sinusoidal radius variations along the axis of the fibre, with spatial frequency $\Omega_{s}$, and corresponding wavelength $\lambda_{s}=2 \pi / \Omega_{s}$.

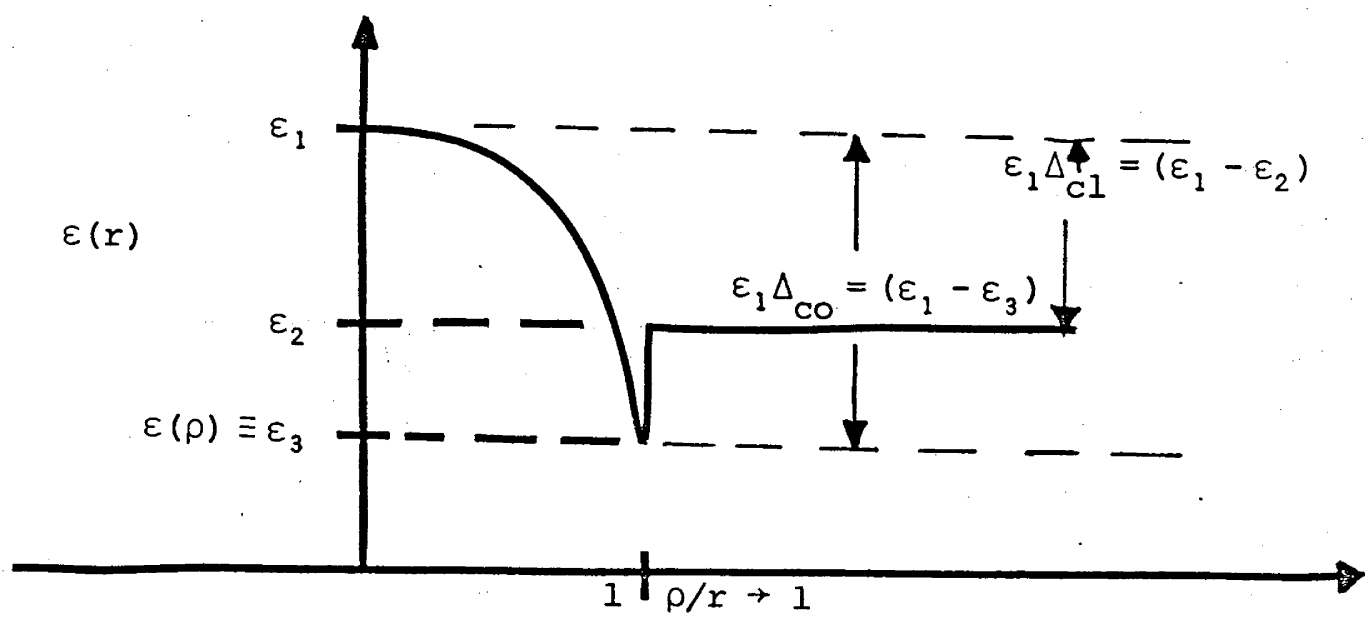

Fig. 10: The dielectric profile of the circular cylindrical dielectric optical fibre. 
where $q\left(r / \rho_{0}\right)$ contains the variation of the dielectric with radius and

$$
\Delta_{c l}=\frac{\left(\varepsilon_{1}-\varepsilon_{2}\right)}{\varepsilon_{1}}
$$

is the maximum relative difference between the core and cladding dielectrics, and

$$
\Delta_{C O}=\frac{\left(\varepsilon_{1}-\varepsilon_{3}\right)}{\varepsilon_{1}}
$$

is the maximum relative difference in the core dielectric variation, and $H(x)$ is the Heaviside step function defined by

$$
\begin{aligned}
H(x) & =0: & & x<0 \\
& =1: & & x>0 .
\end{aligned}
$$

In the section of the fibre with the perturbed core radius, we assume that in every cross-section, the shape of the dielectric profile, determined by $q(x)$ in equation (30), does not change but is only confined to a different radius.

Using the formula for the induced volume current density, equation (1.19) due to this perturbation of the radius, we find

$$
J=i \omega a \rho_{0} \sin \Omega z\left(\frac{\partial \varepsilon}{\partial \rho}\right)_{\rho=\rho_{0}} E_{0}
$$

where we have used a Taylor series expansion around the unperturbed radius $\rho_{0}$, for the dielectric profile of the perturbed waveguide. In a mathematical sense, this expansion does not appear valid around $\rho=\rho_{0}$, where the derivative of $H\left(\rho_{0}-r\right)$ is a delta function.

However, if the fluctuations of the radius are small in comparison to the wavelength of the incident light, the contribution to the volume currents induced due to the core-cladding discontinuity can be considered to be due to a constant field (in the radial direction) and we take this field to be the field at the unperturbed core-cladding interface. In this way, we can represent the core-cladding contribution by a delta function representation which reduces the volume current 
density to a surface current density located at the core-cladding interface.

When the length of the perturbed fibre section is sufficiently large so as to produce distinct (sharp) radiation lobes, ${ }^{+}$.e. when $\mathrm{k}_{2} \mathrm{~L} \gg \mathrm{I}$, the total radiated power $\mathrm{P}_{l \mathrm{~m}^{\prime}}^{\mathrm{R}}$ induced by an incident modal

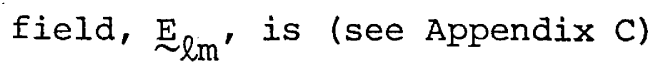

$$
P^{R}=\pi L R^{2} C\left(\omega a \rho_{0}\right)^{2} \int_{0}^{2 \pi} \operatorname{d\phi }\left\{|\underset{\sim}{\hat{R}} \times I|_{\theta_{0}}^{2}\right\}
$$

where $\mathrm{C}$ is defined in equation (1.21). The subscript $\theta_{0}$ on the integrand indicates that the term is to be evaluated for $\theta=\theta_{0}$, where $\theta_{0}$ is the direction of the radiation, to the $z$-axis

$$
\theta_{0}=\cos ^{-1}\left(\frac{\beta_{l m}-\Omega}{k_{2}}\right)
$$

where $\beta_{\ell m}$ is the propagation constant of the bound mode.

The term $I$ in the integrand yields the magnitude of the radiation field (see Appendix $C$ ) and

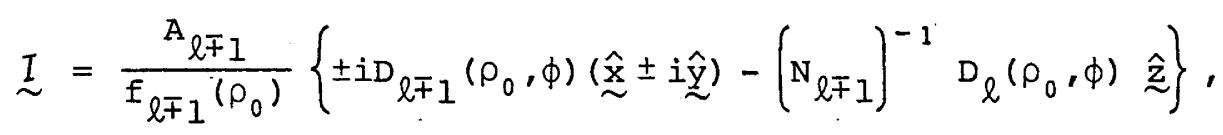

where

$$
\begin{aligned}
D_{p}\left(\rho_{0}, \phi\right)=\int_{0}^{\rho_{0}} r d r \int_{0}^{2 \pi} d \phi^{\prime} & {\left[f_{p}(r)\left(\frac{\partial \varepsilon}{\partial \rho}\right)_{\rho_{0}}\right.} \\
& \left.\times \exp \left\{i\left(k_{2} r \sin \theta \cos \left(\phi-\phi^{\prime}\right)-p \phi^{\prime}\right)\right\}\right] .
\end{aligned}
$$

From an investigation of equation (33) it is observed that there is negligible length dependent radiation loss from the mode lm [9], unless the spatial frequency of the perturbation, $\Omega$, satisfies

$$
\beta_{\ell m}-k_{2} \leqslant \Omega \leqslant \beta_{l m}+k_{2} .
$$

\footnotetext{
$\dagger 2 \mathrm{~L}$ cannot be infinitely large as the incident field of the unperturbed guide would no longer be a valid approximation to the field in the perturbed fibre.
} 
The spatial frequencies, $\Omega$, not satisfied by equation (36), do not induce significant radiation from the waveguide. As snyder points out, such frequencies do induce a small radiation component inversely proportional to $L$ due to the discontinuity at the terminations of this finite element [9]. Such frequencies, however, can still induce coupling between the bound modes of the waveguide and by this procedure the power of the one mode, unable to couple directly to the radiation field, can eventually couple indirectly.

By inspection of equation (16), the term $(\partial \varepsilon / \partial \rho)_{\rho=\rho_{0}}$ can be seen to play a significant rôle in type of radiation expected. Using equation (11) for $\varepsilon(r)$, we observe that, ${ }^{\dagger}$

$$
\left(\frac{\partial \varepsilon}{\partial \rho}\right)_{\rho=\rho_{0}}=\varepsilon_{1}\left(\Delta_{C l}-\Delta_{C O}\right) \delta\left(\rho_{0}-r\right)+\varepsilon_{1} \Delta_{C O} q^{\prime}\left(\frac{r}{\rho_{0}}\right) \frac{r}{\rho_{0}^{2}} H\left(\rho_{0}-r\right),
$$

where the ' indicates the difference with respect to the argument $\left(r / \rho_{0}\right)$.

The first term on the right hand side of equation (37) gives rise to surface current densities located at the core-cladding interface. This is the only term present for the well-known result of the stepindex fibre $[9,5]$. If the dielectric profile is continuous across the core boundary, i.e.

$$
\Delta_{\mathrm{CO}}=\Delta_{\mathrm{Cl}}
$$

Then the surface current contribution to the radiation field does not appear.

The second term on the right hand side of equation (37) gives rise to the volume current density throughout the core of the waveguide. For the continuous profile discussed above, this is the only term that contributes to the radiation field. In general, both surface and volume

$\dagger$ The appearance of the delta function appears to invalidate the Taylor series expansion. However, we use this notation for the particular case of small amplitude variations in comparison to the wavelength of light in the medium. The effect of the discontinuity across the core-cladding boundary can then be considered as occurring at the boundary itself, so that the boundary contributions in this particular case can be written as in equation (37) as we have discussed previously. 
current densities occur. However, in a graded-index fibre, with a discontinuity in the dielectric profile across the core boundary, the modes far from cutoff have negligible intensity at $r=\rho_{0}$, due to the rapid decay of the evanescent field beyond the outer caustic, and the dominant contribution of the radiation field should be due to the volume current distribution in the core.

Let us now consider the situation of a sinusoidal perturbed radius of a weakly guiding. step-index fibre. Using the complete fields, including the small longitudinal fields, the modal power loss per unit length of the $H E_{11}$ mode, $\alpha_{11}$, is [20]

$$
\begin{array}{r}
\alpha_{11}=\frac{\pi a^{2} w^{2} v^{2}}{8 k_{2} \rho^{2} J_{1}^{2}(U)}\left\{J_{0}^{2}(U) J_{0}^{2}\left(k_{2} \rho \sin \theta_{0}\right)+\left(\cos \theta_{0} J_{0}(U) J_{0}\left(k_{2} \rho \sin \theta_{0}\right)\right.\right. \\
\left.\left.+\frac{U}{\beta \rho} \sin \theta_{0} J_{1}(U) J_{1}\left(k_{2} \rho \sin \theta_{0}\right)\right)^{2}\right\}
\end{array}
$$

where $\theta_{0}$ is the radiation angle defined by equation (33).

This result, equation (38), does not agree with Snyder's result [9] even for the forward directed radiation due to an algebraic error. Rawson [12], using a similar approach to the Volume current Method, limits himself to the unphysical study of very short lengths of fibre in which is at most four wavelengths of the spatial perturbation. He thus finds very poorly defined radiation lobes, and consequently cannot predict the effect of radius fluctuations in practical optical waveguides. His approach attempts in an ad hoc fashion to introduce the effect of the core-cladding interface by defining a transmission coefficient $T\left(\theta_{z}\right)$ independent of the location of the imperfection, where

$$
\begin{aligned}
T\left(\theta_{z}\right) & =0 & \theta_{z}<\theta_{C} \\
& =1 & \theta_{z}>\theta_{C} .
\end{aligned}
$$

Although such a restriction may provide an improvement to the total radiated power for highly directional radiation, it should not improve the accuracy of the radiation spectrum since the effect of the core-cladding interface on rays incident from the core at angles $\theta_{\mathbf{z}}>\theta_{\mathbf{z}}$ is to only partially refract the ray and hence the core boundary 
introduces an additional phase term in the radiation field induces addition interference between components of the radiation field.

Furthermore, the ad hoc assumption that the amount of scattered radiation escaping from the core is independent of its location is false as will be shown in Chapter 4.

Let us now discuss the absolute accuracy of the Volume current calculations. To do this, we have plotted in fig. 11 the normalised modal power attenuation coefficients of the $\mathrm{HE}_{11}$ mode calculated by the Volume Current Method and exact Coupled Mode Theory (i.e. the true radiation modes of the fibre are used).

The plot of $\bar{\alpha}$ is for a fibre with $\mathrm{V}=2.4$ and a critical angle $\theta_{C}=0.1$ radians $\left(\sim 6^{\circ}\right)$. The solid curve represents the exact coupled Mode result which is an extension of equation 3.3 .6 of ref. 5 to include the longitudinal field components. The dotted line represents the Volume Current result equation (38) and for $\theta_{0}=0, \bar{\alpha}=0.329$.

For radiation escaping at angles $\theta_{0} \leqslant 3 \theta_{c}$ the Volume Current Method is very inaccurate. This result is to be expected since radiation escaping at small angles relative to the waveguide axis should be most strongly influenced by the presence of the core-cladding interface, which is neglected in the volume current Method. However, for $\theta_{0}>3 \theta_{C^{\prime}}$ excellent agreement between the two methods is displayed.

From this study, and from the obvious physical disabilities of the Volume Current Method, the conclusion should be drawn that the Volume Current Method proves to be an excellent tool for radiation that is not paraxially directed. For paraxial radiation the exact coupled Mode Theory must be used. (We discuss this further in Chapter 4 in which a correction to the Volume Current Method is postulated, which includes the effect of the core-cladding interface.) 


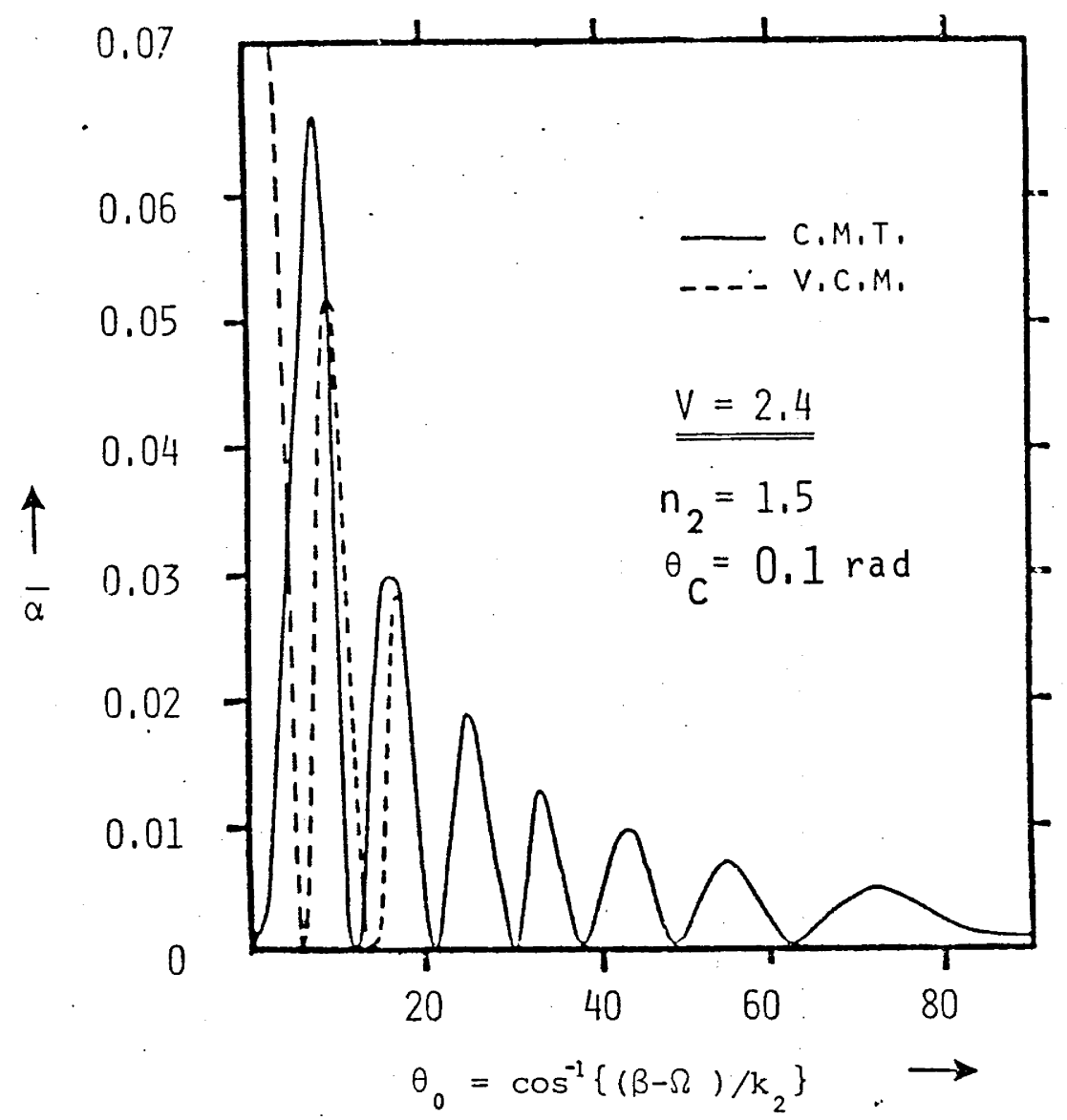

Fig. 11: A plot of the non-dimensional power attenuation coefficients for the $\mathrm{HE}_{11}$ mode for a sinusoidally perturbed core-cladding interface, $\rho(z)=\rho(1+a \sin \Omega z), \bar{\alpha}=\rho \alpha / a^{2}$, where $\rho$ is the unperturbed fibre core radius. $P(z) / P(0)=\exp (-\alpha z)$. The solid line is the result by exact C.M.T. whereas the broken line represents the V.C.M. calculation. 


\section{APPENDIX A \\ THE ELECTROMAGNETIC FIELDS INDUCED BY ELECTRIC AND MAGNETIC CURRENTS}

Let us denote the fields generated by the electric current density ${\underset{\sim}{e}}_{e}$ by $\underset{\sim}{\mathrm{E}_{e}}, \mathrm{H}_{e}$ and similarly for the magnetic current density, $\stackrel{\mathrm{E}}{\sim} \mathrm{m}_{\sim}^{\prime} \underset{\mathrm{H}}{\mathrm{H}}$. The resultant fields generated by a general current density of both electric and magnetic characteristics is obtained by superposition of these two sets of independent fields.

The $\underset{\sim}{\mathrm{E}} \stackrel{\mathrm{H}}{\sim}$ fields satisfy

$$
\begin{aligned}
& \nabla \times \underset{\underbrace{}_{e}}{\mathrm{E}_{e}}=-i \omega \mu(\underline{\sim}) \underset{\sim}{\mathrm{H}}
\end{aligned}
$$

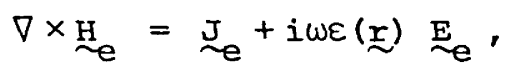

whereas the $\underset{\sim}{\mathrm{E}} \stackrel{\sim}{\mathrm{m}}_{\mathrm{m}}^{\mathrm{H}}$ fields satisfy

$$
\begin{aligned}
& \nabla \times \underset{\sim m}{\mathrm{E}}=-{\underset{\sim}{\mathrm{J}}}_{\mathrm{m}}^{\mathrm{E}}-i \omega \mu(\underset{\sim}{\underline{r}}){\underset{\sim}{\mathrm{m}}}^{\mathrm{H}} \\
& \nabla \times \underset{\sim m}{\mathrm{H}}=i \omega \varepsilon(\underset{\sim}{\sim}) \stackrel{\sim}{\mathrm{m}}_{\mathrm{m}}^{\mathrm{E}} .
\end{aligned}
$$

Let us first consider the fields generated by $\mathrm{J}_{e} \cdot$ From equations (A.I) and (A.2) it follows that $\underset{\sim}{\mathrm{E}}$ satisfies

$$
\begin{aligned}
& \nabla \times \nabla \times \underset{\sim_{e}}{E}=-i \omega \nabla \times(\mu \mathrm{H}) \\
& =-i \omega \mu \underset{J_{e}}{J}+k^{2} \underset{e}{E}-i \omega(\nabla \mu) \times \underset{f_{e}}{H},
\end{aligned}
$$

where $(\nabla \mu)$ signifies the operation $\nabla$ is restricted to $\mu . k \underset{\sim}{(r)}$ is the wavenumber of the light at position $\underset{\sim}{\sim}$, and is

$$
\mathrm{k}^{2}=\omega^{2} \varepsilon \mu .
$$


Upon substitution for $\underset{\sim}{\mathrm{H}}$ from (A.I) into (A.5) we find

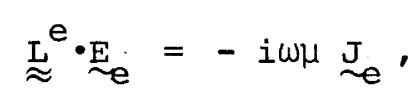

where $\underset{\approx}{\stackrel{e}{\approx}}$ is the operator defined by

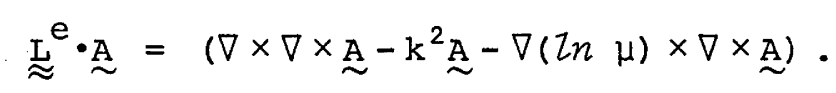

Since $\underset{\sim}{\mathrm{E}}$ is linearly related to $J_{e}$ in (A.7) we can write the solution for $\underset{\sim}{\mathrm{E}}$ in a dyadic Green's function form via

$$
\left.\underset{\sim}{E}(r)=-i \omega \int \mu\left(\underline{x}^{\prime}\right) \underset{\approx}{\Gamma}\left(\underline{r}, \underline{x}^{\prime}\right) \cdot \underset{J_{e}}{J} \underline{\sim}^{\prime}\right) d V^{\prime},
$$

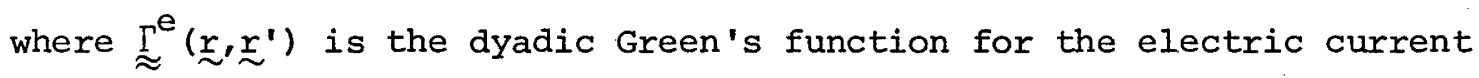
and satisfies

$$
\underset{\approx}{\stackrel{e}{d}} \stackrel{\Gamma^{e}}{\approx}=\delta\left(\underline{r}-\underline{r}^{\prime}\right)
$$

subject to the appropriate boundary conditions for the structure under consideration. $\underset{\approx}{\mathrm{U}}$ is the identity matrix defined by $\underset{\approx}{\mathrm{U}} \cdot \underset{\sim}{A}=\underset{\sim}{A}$ and $\delta\left(\underline{\underline{r}}-\underline{\sim}^{\prime}\right)$ is the usual Dirac delta function.

From (A.1) we see that

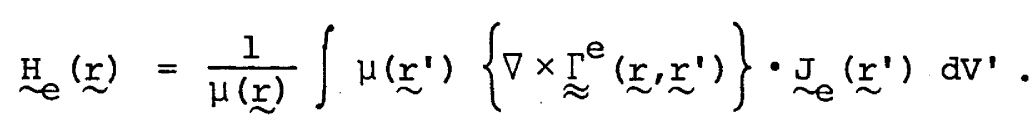

By a similar argument for equations (A.3), (A.4), we find

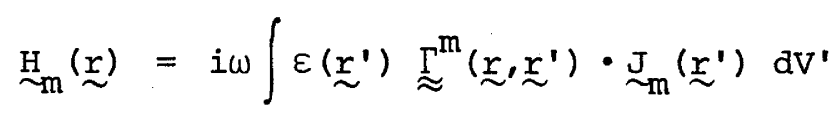

and using (A.4)

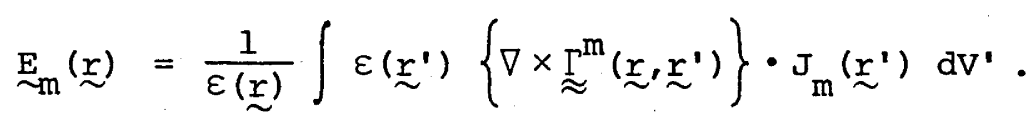

$\underset{\approx}{m}\left(\underset{\sim}{\sim}, \underline{r}^{\prime}\right)$ is the dyadic Green's function for the magnetic current and satisfies

$$
\underset{\approx}{L^{\mathrm{m}}} \cdot \stackrel{\Gamma}{\mathrm{r}}^{\mathrm{m}}=\underset{\approx}{\mathrm{U}} \delta\left(\underset{\sim}{\sim}-\underline{r}^{\prime}\right)
$$

with the boundary conditions appropriate to the structure under 
consideration, where

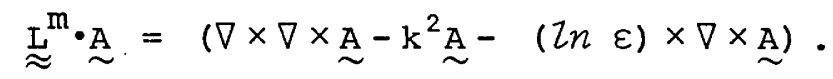

The resultant field for a general distribution of electric and magnetic current densities then is the sum of the independent fields from equations (A.9), (A.11), (A.12) and (A.13) which yield

$$
\begin{aligned}
& \underset{\sim}{E}(\underset{\sim}{)}=\underset{\sim}{E}+\underset{\sim m}{E} \\
& =-i \omega \int \mu\left(\underline{\sim}^{\prime}\right) \underset{\sim}{\stackrel{\Gamma}{\approx}}\left(\underline{r}, \underline{\sim}^{\prime}\right) \cdot \underset{\sim}{J}\left(\underline{r}^{\prime}\right) d V^{\prime}
\end{aligned}
$$

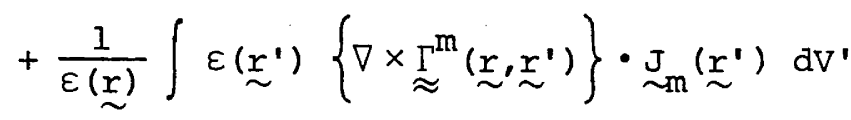

and

$$
\begin{aligned}
& \underset{\sim}{H}(\underline{r})=\frac{1}{\mu(\underline{r})} \int \mu\left(\underline{\sim}^{\prime}\right)\left\{\nabla \times{\underset{\approx}{\Gamma}}^{e}\left(\underline{r}_{\sim}, \underline{r}^{\prime}\right)\right\} \cdot \underset{\sim}{J}\left(\underline{r}^{\prime}\right) d V^{\prime} \\
& +i \omega \int \varepsilon\left(\underline{\underline{r}}^{\prime}\right) \underset{\approx}{\stackrel{\Gamma}{\approx}}\left(\underline{r}, \underline{\underline{r}}^{\prime}\right) \cdot{\underset{\sim m}{J}}_{m}\left(\underline{\underline{r}}^{\prime}\right) d V^{\prime} .
\end{aligned}
$$

Thus, we can see that given the current densities $\mathrm{J}_{\mathrm{m}}$ and $\mathrm{J}_{\mathrm{e}}$ together with the dyadic Green's function $\stackrel{\Gamma}{\approx}^{\mathrm{e}} \Gamma^{\mathrm{m}}$ we can completely determine the electromagnetic fields. In general, for arbitrary permittitivity $\varepsilon(r)$ and permeability $\mu(r)$ profiles the dyadic Green's functions are mathematically impossible to handle. However, for the particular case of dielectric optical fibres these profiles vary slowly compared to the wavelength of light and thus where this occurs, the terms in (A.7) and (A.15) that contain $\nabla(\operatorname{In} \mu), \nabla(\operatorname{In} \varepsilon)$ can be neglected, which further simplifies equation (A.16),(A.17) since

$$
\stackrel{\Gamma}{\approx}^{\mathrm{m}}=\Gamma^{\mathrm{e}}=\underset{\approx}{\Gamma}
$$

where $\underset{\approx}{\Gamma}$ is the dyadic Green's function for the vector Helmholtz equation. This simplifying approximation will be used throughout to maintain consistency with the solutions for the model fields of Chapter 2.

It is interesting to note in passing that the duality of the electromagnetic fields [13] is not retained in inhomogeneous media, except when all derivatives of the permittivity $\varepsilon(r)$ and permeability $\mu(r)$ are ignored. 


\section{APPENDIX B \\ RADIATION FROM AN ISOLATED IRREGULARITY}

The irregularity of volume $V$ and dielectric permittivity, $\varepsilon_{3}$, with dielectric permittivity $\varepsilon(\underset{\sim}{r})$ is located at the position ${\underset{\sim}{\sim}}_{0}$ in the core of the waveguide. With the restriction that the dimensions of the irregularity are small compared to the wavelength of the incident field, the field over the volume of the irregularity can be assumed constant.

The induced volume current density due to an incident mode $\underset{\sim}{\mathrm{E}_{\ell m}}$ can be written as

$$
\underset{\sim}{J}(\underset{\sim}{\sim})=i \omega \Delta \varepsilon(\underset{\sim}{\sim}) \underset{\sim}{E_{l m}} \stackrel{(\underline{r})}{\sim} .
$$

where

$$
\Delta \varepsilon(\underline{r})=\left(\varepsilon_{3}(\underline{r})-\varepsilon(\underline{r})\right),
$$

and the time averaged Poynting vector, $s^{R}$, becomes, from equation $(1.20)$,

$$
S^{R}=(V \Delta \varepsilon \omega)^{2} \quad C\left|\underset{\sim}{\hat{R}} \times \underset{\sim}{E}\left(\underline{x}_{0}\right)\right|^{2}
$$

and thus the total power radiated, $\mathrm{P}^{\mathrm{R}}$, from equation (1.16) and (B.2), is

$$
\begin{aligned}
& P^{R}=R^{2} \int_{0}^{\pi} S^{R} \sin \theta d \theta \\
& =R^{2}(\omega \Delta \varepsilon V)^{2} C \int_{0}^{2 \pi} \int_{0}^{\pi}\left|\underset{\sim}{\hat{R}} \times \underset{\sim m}{E} \underset{\sim}{E}\left(r_{0}\right)\right|^{2} \sin \theta d \theta d \phi .
\end{aligned}
$$

Now writing $\underset{\sim}{\hat{R}}$ in spherical polar co-ordinates with the z-axis parallel to the fibre axis

$$
\underset{\sim}{\hat{R}}=\sin \theta \cos \phi \underset{\sim}{\hat{x}}+\sin \theta \sin \phi \underset{\sim}{\hat{y}}+\cos \theta \underset{\sim}{\hat{z}}
$$

and using equations $(2.2 .42)-(2.2 .50)$ for the normalised modal electric 
field, and writing the field in cartesian co-ordinates, ignoring the longitudinal fields

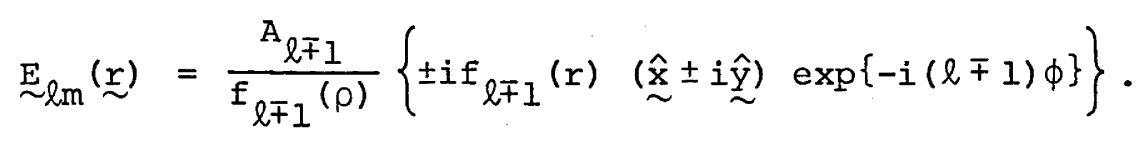

The total radiated power, from equation (B.3) with (B.4) becomes

$$
\mathrm{P}^{\mathrm{R}}=\frac{16 \pi \mathrm{R}^{2}}{3}(\omega \Delta \varepsilon V)^{2} \mathrm{C}\left|\frac{\mathrm{A}_{\ell \mp I} \mathrm{f}_{\ell \mp I}\left(\mathrm{r}_{0}\right)}{\mathrm{f}_{\ell \mp I}(\rho)}\right|^{2},
$$

where $A_{\ell \mp 1}$ is the normalisation coefficient defined by equation $(2.2 .53)$. 


\section{APPENDIX C \\ RADIATION FROM AN OPTICAL FIBRE WITH \\ AXIAL SINUSOIDAL VARIATIONS OF THE CORE RADIUS}

Consider an inhomogeneous dielectric fibre as discussed in Chapter 2. For generality we assume that the dielectric profile is discontinuous at the core-cladding boundary, as in fig. 12. Such a dielectric profile can be described via

$$
\varepsilon(r)=\left\{\varepsilon_{1}\left(1-\Delta q\left(\frac{r}{\rho_{0}}\right)\right)-\varepsilon_{2}\right\} H(\rho-r)+\varepsilon_{2},
$$

where $H(\rho-r)$ is the Heaviside step function defined as

$$
\begin{aligned}
H(x) & =0: & & x<0 \\
& =1: & & x>0
\end{aligned}
$$

and the dielectric variation defined by $q(r / \rho)$ is

$$
\begin{aligned}
q\left(\frac{r}{\rho}\right) & =0: & & r=0 \\
& =1: & & r=\rho .
\end{aligned}
$$

Consider a length $2 L$ of fibre centred at $z=0$, with a sinusoidally perturbed core radius as in fig. 13, viz.

$$
\rho=\rho_{0}(I+a \sin \Omega z)
$$

where $\Omega$ is the spatial frequency of the perturbation a $\rho_{0}$ its amplitude, which is assumed to be much less than the wavelength of the excitation field.

This perturbation will induce a volume current density as defined in equation (1.19), viz. 


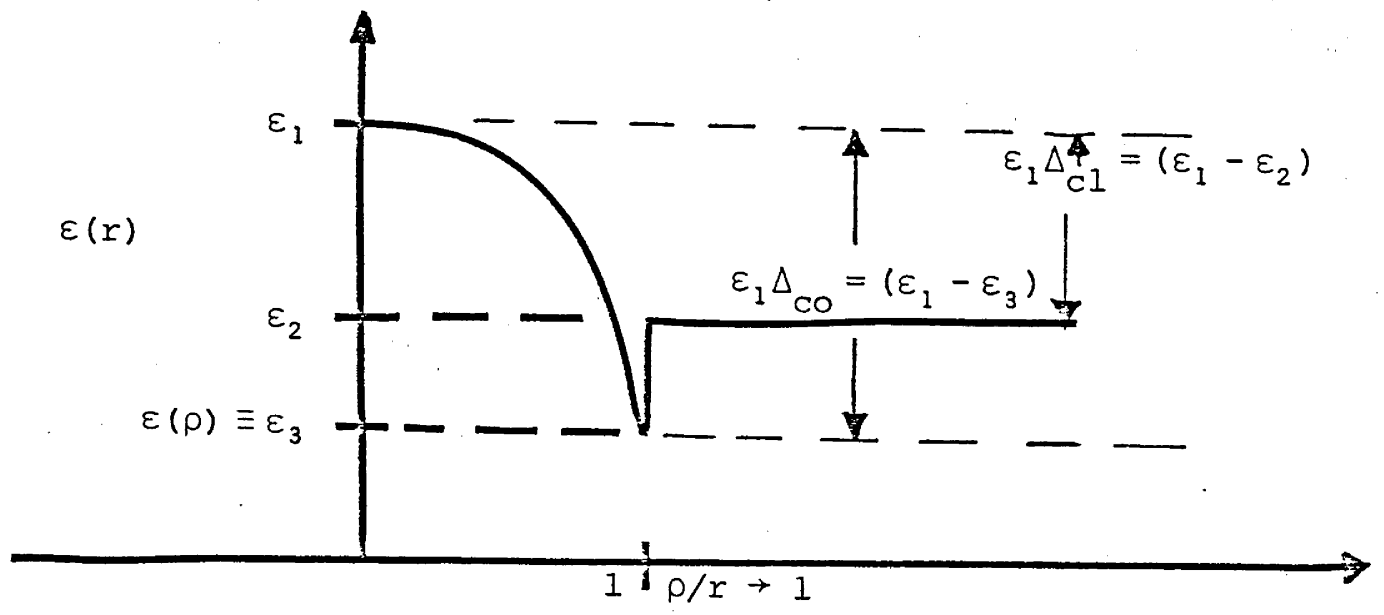

Fig. 12: The dielectric profile of the circular cylindrical dielectric optical fibre. 


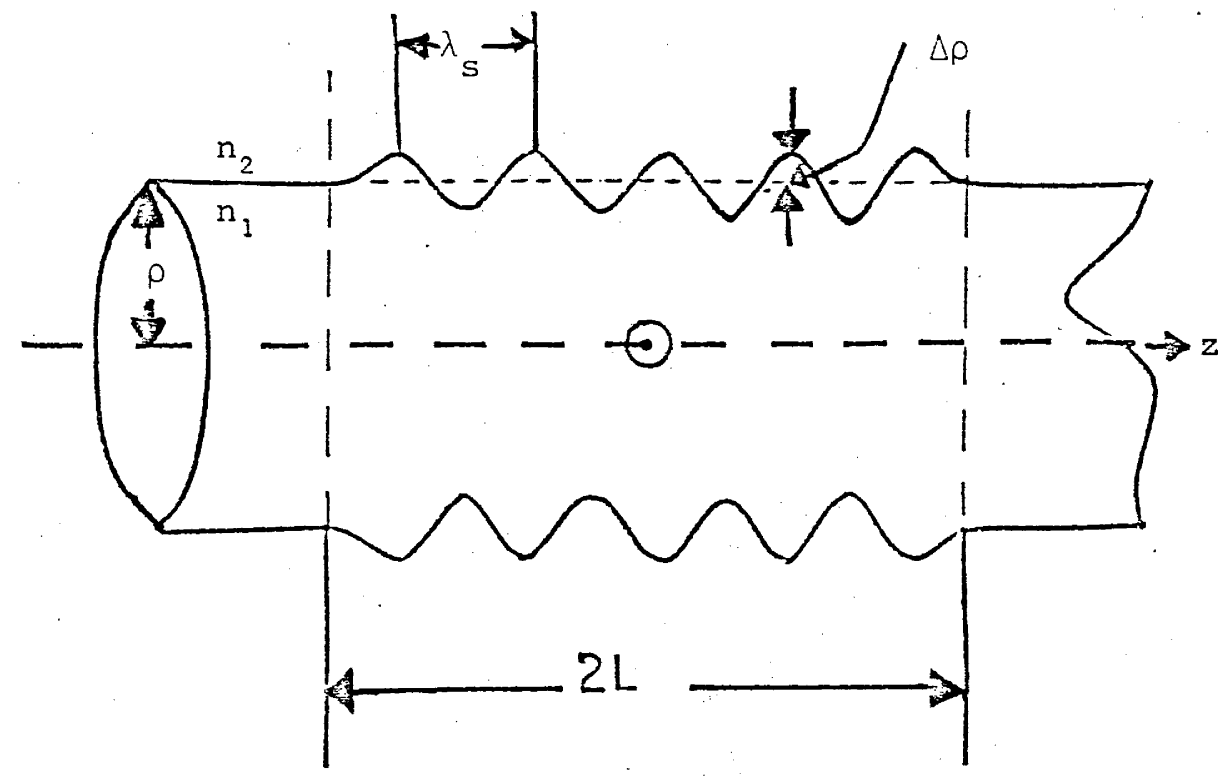

Fig. 13: Sinusoidal radius variations along the axis of the fibre, with spatial frequency $\Omega_{\mathrm{s}}$, and corresponding wavelength $\lambda_{\mathrm{s}}=2 \pi / \Omega_{\mathrm{s}}$. 


$$
\underset{\sim}{J}=i \omega \Delta \varepsilon(r) \underset{\sim}{\mathrm{E}} \underset{\sim}{(r)} \text {. }
$$

Now for sufficiently small variations in the radius, the term $\Delta \varepsilon(r)$ can be approximated by using a Taylor series expansion of $\varepsilon_{\text {pert }}(r / \rho)$ around $\rho=\rho_{0}$ such that

$$
\Delta \varepsilon(r)=a \rho_{0} \sin \Omega z\left(\frac{\partial \varepsilon}{\partial \rho}\right)_{\rho=\rho_{0}}
$$

Now writing $\underset{\sim}{\hat{R}}$ and $\underset{\sim}{\prime}$ in spherical and cylindrical polar co-ordinates respectively, we have

and

$$
\underset{\sim}{R}=\sin \theta \cos \phi \underset{\sim}{x}+\sin \theta \sin \phi \underset{\sim}{y}+\cos \theta \underset{\sim}{z}
$$

$$
\underline{r}^{\prime}=r \cos \phi^{\prime} \underset{\sim}{\hat{x}}+r \sin \phi^{\prime} \underset{\sim}{\hat{y}}+z^{\prime} \hat{z}
$$

and

$$
\underline{\mathrm{R}}_{\underline{x}^{\prime}}^{\prime}=r \sin \phi \cos \left(\phi^{\prime}-\phi\right)+z^{\prime} \cos \theta \text {. }
$$

The time averaged Poynting vector of the radiation from the incident mode $\underset{\ell_{\mathrm{m}}}{\mathrm{E}_{\mathrm{m}}}$ from equation $(1.20)$ can be written as

where

$$
s^{R}=\left(a \omega \rho_{0}\right)^{2} C\left\{\frac{\sin k_{2} \gamma_{+} L}{k_{2} \gamma_{+}}+\frac{\sin k_{2} \gamma_{-} L}{k_{2} \gamma_{-}}\right\}^{2}|\underset{\sim}{\hat{R}} \times \underset{\sim}{I}|^{2}
$$

$$
\mathrm{k}_{2} \gamma_{ \pm}=\mathrm{k}_{2} \cos \theta-\beta_{\ell m} \pm \Omega
$$

Using equations $(2.2 .43)-(2.2 .50)$ for the modal electromagnetic field, we can write $\underset{\sim}{\sim}$ as

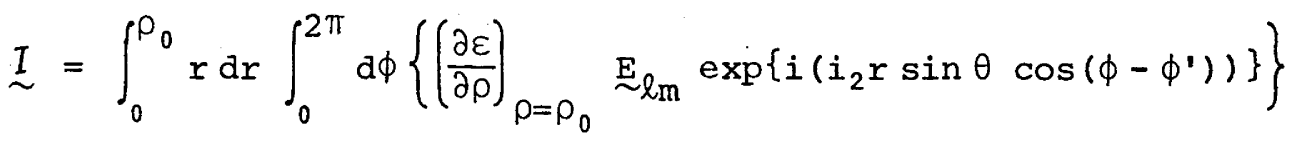

$$
\begin{aligned}
& =\frac{A_{\ell \mp 1}}{f_{\ell \mp 1}\left(\rho_{0}\right)}\left\{ \pm i D_{\ell \mp 1}\left(\rho_{0}, \phi\right)(\underset{\sim}{\hat{x}}+i \underset{\sim}{\hat{y}})-\frac{1}{N_{\ell \mp 1}} D_{\ell}\left(\rho_{0}, \phi\right) \underset{\sim}{\hat{z}}\right\},
\end{aligned}
$$

where

$$
D_{p}\left(\rho_{0}, \phi\right)=\int_{0}^{\rho_{0}} r d r \int_{0}^{2 \pi} d \phi\left[\left(\frac{\partial \varepsilon}{\partial \rho}\right)_{\rho=\rho_{0}} f_{p}(r) \exp \left\{i\left\{k_{2} r \sin \theta \cos \left(\phi-\phi^{\prime}\right)-p \phi\right\}\right\}\right]
$$

the total power radiated, $\mathrm{P}_{\ell^{\prime}}^{\mathrm{R}}$ ' from the incident mode ${\underset{\sim}{\mathrm{I}}}_{\mathrm{m}}$ due to this perturbation is 


$$
\begin{aligned}
& P_{l m}^{R}= R^{2} \int_{0}^{2 \pi} d \phi \int_{0}^{\pi} \sin \theta d \theta s^{R} \\
&=\left(a \omega \rho_{0}\right)^{2} C R^{2} \int_{0}^{2 \pi} d \phi \int_{0}^{\pi} d \theta \sin \theta\left[\left\{\frac{\sin ^{2}\left(k_{2} \gamma_{+} L\right)}{\left(k_{2} \gamma_{+}\right)^{2}}+\frac{\sin ^{2}\left(k_{2} \gamma_{-} L\right)}{\left(k_{2} \gamma_{-}\right)^{2}}\right\}\right. \\
&\left.+\frac{2 \sin \left(k_{2} \gamma_{+} L\right) \cdot \sin \left(k_{2} \gamma_{-} L\right)}{k_{2}^{2} \gamma_{+} \gamma_{-}}|\underset{\sim}{\hat{R}} \times \underset{\sim}{I}|^{2}\right]
\end{aligned}
$$

Now for $k_{2} L \gg 1$ (but $L$ not too large, so that the incident modal field is still a valid approximation to the field over the length of the perturbed fibre), we find [9]

$$
\int_{-a}^{b} \frac{\sin ^{2} L x}{x^{2}} G(x) d x=L \pi G(0)+O\left(\frac{1}{L}\right)
$$

if $\mathrm{a}>0$ and $\mathrm{b}>0$, i.e. $\sin ^{2} \mathrm{Lx} / \mathrm{x}^{2}$ behaves like a delta function. Now transforming from $\theta$ to $\gamma_{\mp}$

$$
d \gamma_{\mp}=-\sin \theta d \theta
$$

and the limits of the integrand become

and

$$
\gamma_{\mp}(\theta=0)=\frac{1}{k_{2}}\left(k_{2}-\beta+\Omega\right)
$$

$$
\gamma_{\mp}(\theta=\rho)=\frac{1}{k_{2}}\left(-k_{2}-\beta \mp \Omega\right)
$$

Now if these limits are either side of the points where $\gamma_{\mp}=0$, there is a dominant length dependent radiation varying linearly with the length of the perturbed fibre. Thus it is of fundamental significance to locate the zeros of $\gamma_{\mp}$

$$
\mathrm{k}_{2} \gamma_{\mp}=0=\mathrm{k}_{2} \cos \theta-\beta \mp \Omega
$$

Since the incident mode is a bound mode of the unperturbed fibre,

$$
\beta_{l m}-k_{2}>0
$$

and since $\Omega$, the spatial frequency of the perturbation must be positive 


$$
\Omega>0
$$

$\gamma$ has no real zeros

$\gamma_{+}$has real zeros if $\Omega$ lies in the range of spatial frequencies

$$
\beta_{\ell m}-k_{2} \leqslant \Omega \leqslant \beta_{\ell m}+k_{2}
$$

and these zeros are located at angles $\theta_{0}$ to the z-axis, where

$$
\theta_{0}=\cos ^{-1}\left(\frac{\beta_{l m}-\Omega}{k_{2}}\right) \text {. }
$$




\section{REFERENCES}

[1] A.W. Snyder: "Coupling of modes on a tapered cylinder", I.E.E.E. Trans. MTT-18, 383 (1970).

[2] A.W. Snyder: "Mode propagation on optical waveguides", Electron. Letters 6, 561 (1970).

[3] A.W. Snyder: "Mode propagation in a non-uniform cylindrical medium", I.E.E.E. Trans. MTT-19, 402 (1971).

[4] A.W. Snyder: "Coupled mode theory of optical fibers", J. Opt. Soc. Am. 62, 1267 (1972).

[5] D. Marcuse: Theory of Dielectric Optical Waveguides (Academic Press, New York, 1974).

[6] G.L. Yip: "Launching efficiency of the $\mathrm{HE}_{11}$ surface wave mode on a dielectric rod", I.E.E.E. Trans. MTT-18, 1034 (1970).

[7] G.L. Yip, J. Martucci and G.W. Farnell: "Scattering loss in a cladded optical waveguide", Electron. Letters 9, 293 (1973).

[8] G.L. Yip and J. Martucci: "Scattering from a localised inhomogeneity in a cladded fiber optical waveguide. 1: Radiation loss", Appl. Optics 15, 2131 (1976).

[9] A.W. Snyder: "Radiation losses due to variations of radius on dielectric or optical fibers", I.E.E.E. Trans. MTT-18, 608 (1970).

[10] P.J.B. Clarricoats and K.B. Chan: "Propagation behaviour of cylindrical-dielectric-rod waveguides", Proc. I.E.E.E. 120, 1371 (1973).

[11] K.B. Chan: Electromagnetic Wave Propagation in Optical Waveguides, Ph.D dissertation, Univ. of London (1972).

[12] E.G. Rawson: "Analysis of scattering from fiber waveguides with irregular core surfaces", Appl. Optics 13, 2370 (1974).

[13] C.H. Papas: Theory of Electromagnetic Wave Propagation (McGrawHil1, New York, 1965).

[14] Ref. 5, p.87 and p.168. 
[15] C.M. Angulo and W.S.C. Chang: "A variational expression for the terminal admittance of a semi-infinite dielectric rod", I.R.E. Trans. AP-7, 207 (1959).

[16] A.W. Snyder: "Scattering due to irregularities on dielectric or optical fibres", Electron. Letters $\underline{5}$, no. 12 (1969).

[17] A.W. Snyder and D.J. Mitchell: "Whispering gallery rays within dielectric circles and spheres", Electron. Letters 10, 16 (1974).

[18] Ref. 13, equation (82), p.33. (This book uses time variation $\left.e^{-i \omega t}.\right)$

[19] A.W. Snyder: "Leaky-ray theory of optical waveguides of circular cross section", Appl. Physics 4, 273 (1974).

[20] D. Marcuse: "Radiation loss of $\mathrm{HE}_{11}$ mode of a fiber with sinusoidally perturbed core boundary", Appl. Optics 14, 3021 (1975).

[21] I.A. White and A.W. Snyder: "Radiation from dielectric optical waveguides: A comparison of techniques", Appl. Optics (1977).

[22] Ref. 5, equation (4.7-5), p.168.

[23] Ref. 5, equation (4.7-1), p.168.

[24] Ref. 5, equations (4.7-14-15), p.169.

[25] R.H.T. Bates: "Analytic constraints on electromagnetic theory field computations", I.E.E.E. Trans. MTT-23, 605 (1975).

[26] Ref. 5, Chapter 5, p.216.

[27] M. Abramowitz and I. Stegun: Handbook of Mathematical Functions (Dover, New York, 1970), Chapter 11.

[28] D.S. Jones: Theory of Electromagnetism (Pergamon, Oxford, 1964).

[28] A.W. Snyder: "Power loss on Optical Waveguides", Proc. I.E.E.E. 60, no. 6, 757 (1972). 


\section{CHAPTER 4 \\ CORRECTIONS TO THE VOLUME CURRENT METHOD}

\subsection{INTRODUCTION}

In Chapter 3 we presented the conceptually simple Volume Current Method for the determination of the radiative losses from weakly guiding dielectric waveguides. The success of the Volume Current Method is predicated on the fact that the dielectric difference between the core and cladding is small. This small dielectric difference ensures that only radiation in a small fraction of the total solid angle into which radiation emanates is significantly affected by the discontinuity in the dielectric permittivity. Radiation emanating from a source within the core of the dielectric waveguide is not significantly affected by the dielectric difference if its angle of inclination to the waveguide axis $\theta_{z}$ satisfies $^{\dagger}$

$$
\theta_{z}>\theta_{c}=\sin ^{-1}\left(1-\frac{\varepsilon_{2}}{\varepsilon_{1}}\right)^{\frac{1}{2}},
$$

where $\varepsilon_{1}$ and $\varepsilon_{2}$ are the core and cladding dielectric permittivities, as usual.

However, for radiation emanating from the core with $\theta_{\mathbf{z}} \sim \theta_{\mathbf{c}^{\prime}}$ the core-cladding dielectric difference is significant, as this radiation which is radiated into leaky modes (or rays) of the optical waveguide remains within the core for long distances [1]. In general, it is this near forwardly-directed radiation (i.e. paraxially directed) that is the most significantly affected radiation for the dielectric optical fibre, since these types of radiation mechanism must be the

This statement is true for most leaky rays as long as equation (I) is satisfied. As Snyder and Mitchell show [1], leaky rays with $\theta_{z}>2 \theta_{C}$ rapidly radiate away from the waveguide core when $V \gg 1$. 
dominant irregularity in optical waveguides, otherwise enormous losspenalties would be suffered [2]. These radiation mechanisms generally arise from a nearest neighbour coupling of modes, as described by Gloge [3]. From the discussion of the philosophy of the Volume Current Method in Chapter 3, we would not expect the Volume Current Method to provide an accurate determination of such radiative losses, since it is this radiation which is strongly affected by the small core-cladding dielectric difference.

Indeed, in Section 3.3.2, the analysis of the Volume Current Method for sinusoidal fluctuations of the radius of a step-index dielectric optical fibre was shown to be totally inaccurate for spatial frequencies of the radius perturbation that induced forward-directed radiation. We reproduce fig. 3.11 here as fig. 1 to allow for easy inspection of this discrepancy. As the spatial frequencies, $\Omega$, that induce forward-directed radiation are small, i.e. $\Omega \sim \beta-k_{2}$ from equation (3.3.36), the spatial wavelength of such perturbations are large and in practice these longer wavelengths are more common due to the drawing process of the optical fibre where machine vibrations induce such fluctuations [4]. Thus, the volume Current Method in its present format does not appear to provide as powerful a tool for analysis of waveguide imperfections as the cumbersome Coupled Mode Theory treatments [5].

However, one is reticent to accept this conclusion due to complexity of normalising radiation modes of even the most simple dielectric waveguide, which is a necessity for the application of Coupled Mode Theory. Therefore, it would be preferable to attempt to correct the volume Current Method in some way, as the analysis by this approach is much simpler than its counterpart.

To this point in time, there are two such proposals for the correction to the Volume Current Method $[6,7]$. Rawson [6] has suggested one such correction which is extremely simple to apply, by which the radiation pattern, over the angle $\theta_{z}$ to the waveguide axis, is truncated below $\theta_{z}=\theta_{C}$ so that all radiation calculated by the volume current Method that propagates at an angle to the axis less than $\theta_{C}$ never enters the radiation field at infinity. This geometric optics correction based 


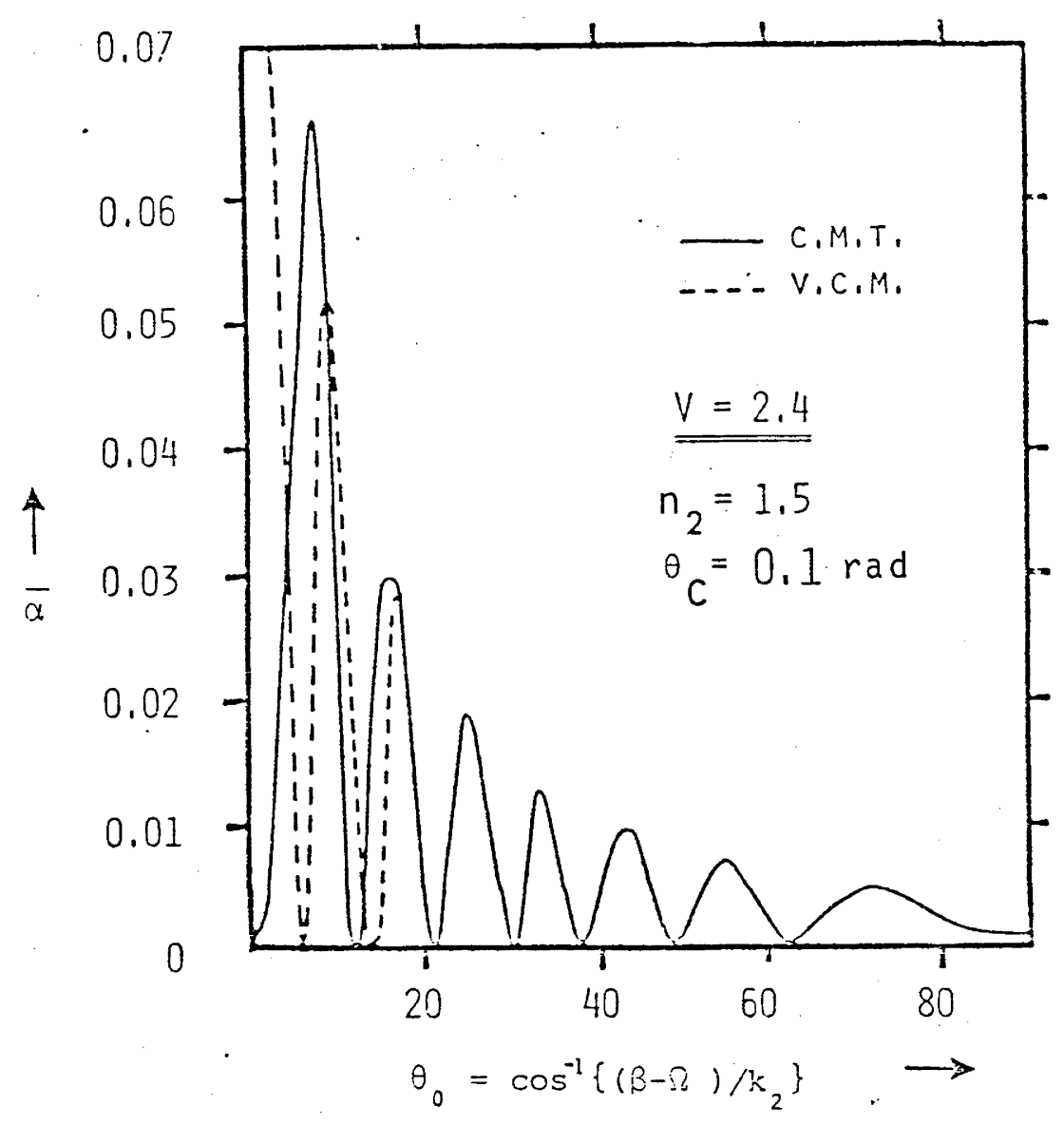

Fig. 1: A plot of the non-dimensional power attenuation coefficients for the $\mathrm{HE}_{11}$ mode for a sinusoidally perturbed core-cladding interface, $\rho(z)=\rho(1+a \sin \Omega z), \bar{\alpha}=\rho \alpha / a^{2}$, where $\rho$ is the unperturbed fibre core radius. $P(z) / P(0)=\exp (-\alpha z)$. The solid line is the result by exact C.M.T. whereas the broken line represents the V.C.M. calculation. 
on the total internal reflection of such rays has a strong physical basis of ignoring the energy that would be totally internally reflected and allowing all other energy to be totally refracted; however, it presupposes that all the radiation mechanisms are within the core (i.e. for $r<p$, the radius of the core), and the application of this criterion becomes somewhat confused for sources on the core-cladding boundary, such as we have just discussed above. Application of the criterion for such loss processes fails to predict the correct loss around the forward direction $[6,7]$.

The second proposed correction technique, postulated by Marcuse, in his text on optical waveguide theory [7], is rather ad hoc. The correction is made by replacing the transverse wavenumber of the radiation in the cladding, $Q_{r}$, by the transverse wavenumber of the radiation in the core $U_{r}\left\{=\left(V^{2}+Q_{r}^{2}\right)\right\}^{\frac{1}{2}}$, and its justification is that for weak dielectric optical fibres, these two parameters are indistinguishable. However, from an investigation of their forms, we find

and

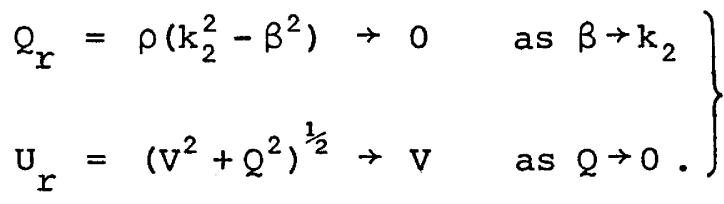

Since $B / k_{2}=1$ corresponds to the forward-directed radiation, we readily observe that this correction has no justification for forward-directed radiation. As $\beta \rightarrow 0, i . e$. as the radiation becomes more transversely directed $U / Q \rightarrow 1$, so that for this radiation Marcuse's correction technique does provide an excellent answer.

For most radiation emanating from within the core, the Volume Current Method can still be used even for highly directed radiation by directly appealing to Rawson's correction or to a more sophisticated version of the correction by including leakage coefficients for the rays with $\theta_{z} \sim \theta_{C}$ [1] to correct for the slowly attenuating tunnelling rays. Although such a correction will not provide an accurate radiation pattern due to the neglect of the interference between the slowly refracting or tunnelling rays, it will provide an accurate determination of the total power radiated, since eventually all energy propagating at angles $\theta_{z}>\theta_{C}$ radiates away from the core. 
Thus, the only radiation mechanisms which appear to critically depend on the dielectric difference between the core and the cladding are forward-directed radiation loss processes emanating from, on or beyond the core-cladding interface. In this chapter, we will concern ourselves with such loss processes and to clarify the principles and facilitate the analysis. We shall consider only step-index waveguides.

The basis of the correction technique is the electrodynamic equivalent of electromagnetic image techniques used in statics problems [8]. In section 2, we present the philosophy of the correction and apply the correction to the situation of a plane wave incident on a dielectric interface between two homogeneous dielectrics with a sinusoidal perturbation to the interface.

In section 3, we shall demonstrate the application of the correction to waveguides for the elementary symmetric step-index slab waveguide with sinusoidal fluctuation of the waveguide thickness. Lastly, we shall present the further extension of this analysis for the cylindrical dielectric optical fibre of circular cross section, with sinusoidal radius fluctuations.

\subsection{THE PHILOSOPHY OF THE VOLUME CURRENT METHOD CORRECTION AND APPLICATION TO PLANAR STRUCTURES}

The studies of Sommerfeld, Weyl and Van der Pol [9] on the effect of the earth on propagation of radio waves provide the necessary physical intuition to interpret the effect of the presence of dielectric or magnetic boundaries on radiating sources. Weyl's study in particular demonstrated that the effect on the electromagnetic fields radiating from the source in the presence of a planar boundary can be constructed from the plane wave spectral components of the radiation field. Considering their associated phase change along the path and the reflection and refraction from the interface, Weyl constructed the radiation fields in the upper and lower half spaces separated by the boundary, as in fig. 2 .

Thus, by considering the individual plane wave components of 


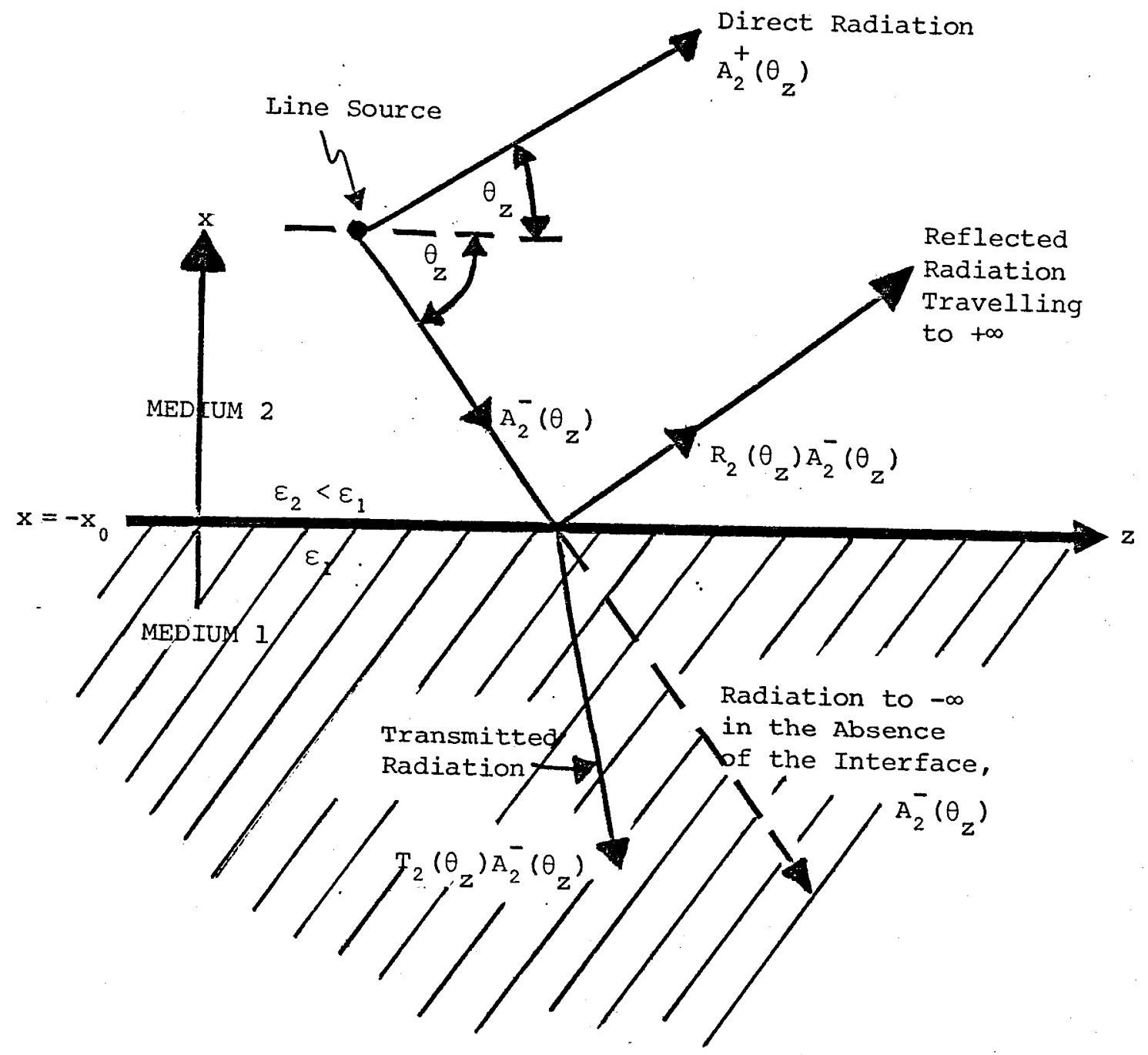

Fig. 2: Radiation from a line source located at the position $x=0$, in the presence of a dielectric interface. 
the two independent polarisations, with amplitudes $\mathrm{A}_{\mathrm{e}, \mathrm{m}}^{+}\left(\theta_{\mathrm{z}}\right)$ and $A_{e, m}^{-}\left(\theta_{z}\right),+$ of the radiation from the radiator in free space, that make angles $+\theta_{z}$ and $-\theta_{z}$ to the $z$-axis respectively, as in fig. 2 , we can readily determine the radiation fields of the radiator in the presence of a boundary between two homogeneous media. If we consider a dielectric interface between two homogeneous dielectrics of dielectric permittivity $\varepsilon_{1}$ and $\varepsilon_{2}$ with $\varepsilon_{1}>\varepsilon_{2}$, where the interface is located at a distance $x_{0}$ below the source at the origin 0 , as in fig. 2 , the plane wave components of the radiation from the source travelling in the positive and negative $\mathrm{x}$ directions becomes,

for $x>0 \quad A_{\text {tot }}^{+}\left(\theta_{z}\right)=A^{+}\left(\theta_{z}\right)+R\left(\theta_{z}\right) A^{-}\left(\theta_{z}\right) e^{-2 i k_{2} x \sin \theta_{z}}$

and

for $x<x_{0} \quad A_{\text {tot }}^{-}\left(\theta_{z}\right)=T\left(\theta_{z}\right) A^{-}\left(\theta_{z}\right) e^{-i k_{2} x_{0} \sin \theta_{z}}$,

where $R\left(\theta_{z}\right)$ and $T\left(\theta_{z}\right)$ are the Fresnel's amplitude reflection and transmission coefficients for the plane waves incident at angle $\theta_{\mathbf{z}}$ to the interface, respectively, for the two independent polarisations. As we are only interested in the radiation fields, we shall not discuss the field for the region between the source and the interface.

From the form of equation (1a) the radiation field from a source in the presence of a boundary consists of the direct radiation from the source to the observation point plus a reflected field from the interface.

In equations (1) not only do we need to consider the Fresnel coefficients but we must also include the phase change along the wave, as can be seen from the terms, $\exp \left\{i k_{2} x_{0} \sin \theta_{z}\right\}$. When the source is located on the dielectric interface, the source behaves as if it were located just inside the boundary of the upper or lower half space for the fields in the upper and lower half space respectively [10], so that for both regions, the field at infinity has the form

\footnotetext{
+ The subscripts $e$ and $m$ refer to the electric or magnetic fields
} polarised perpendicular to the plane of incidence of the plane wave. 


$$
A_{\text {tot }}^{+}\left(\theta_{z}\right)=A_{2}^{+}\left(\theta_{z}\right)+R_{2}\left(\theta_{z}\right) A_{2}^{-}\left(\theta_{z}\right)
$$

and

$$
A_{\text {tot }}^{-}\left(\theta_{z}\right)=A_{1}\left(\theta_{z}\right)+R_{1}\left(\theta_{z}\right) A_{1}^{+}\left(\theta_{z}\right)
$$

where the subscripts 1 and 2 refer to the plane wave amplitudes of the radiator in media 1 and 2 respectively, and $R_{\frac{1}{2}}\left(\theta_{z}\right)$ is the plane wave Fresnel reflection coefficient for the plane wave incident on the interface with angle $\theta_{z}$ to the interface, from the upper or lower mediums respectively.

These equations form the basis of the Volume Current Method correction for scatterers located on the core-cladding interface. As the Volume Current Method treats all dielectric irregularities in the waveguide as current sources in the infinite homogeneous dielectric medium, we can see that from equations (1) and (2) we could correct all two-dimensional waveguide radiation analyses very simply by incorporating the planar reflection coefficients, for the plane wave components, from the waveguide structure. Directly from equations (2) we can see one important result for isotropic scattering processes. For radiation emanating paraxially, the reflection coefficient $R\left(\theta_{z}\right) \sim-1$ so that for $A^{-}\left(\theta_{z}\right)=A^{+}\left(\theta_{z}\right)$, the near forward-directed radiation is zero. From our discussion in Chapter 3 , this direction provides the most significant error of the Volume Current calculations. Thus, we have now provided an elementary explanation of this discrepancy by the interpretation of the interference between the direct radiation from the irregularities and the reflected radiation from the surface.

To extend the above analysis to cylindrical waveguides, it would appear that one should rederive, in all generality, Weyl's solution for such waves. However, from the consideration of the physical and mathematical meaning of the Fresnel coefficients, it is not necessary to attempt this extension. As the Fresnel amplitude reflection and transmission coefficients are the values of the arbitrary constants for the plane wave components that exist on either side of the boundary that satisfy the electromagnetic boundary conditions, any composite field constructed from the plane wave components together with their associated Fresnel coefficients must necessarily satisfy the 
boundary conditions of the structure.

If we could generate electromagnetic cylindrical wave reflection coefficients analogous to these coefficients for reflections and employ the cylindrical wave decomposition of the radiation fields due to sources we could then use an analogous approach, as we have outlined above for sources in the presence of cylindrical boundaries. In section 4 we will discuss this approach.

In this cylindrical wave situation, the waves analogous to positive and negative travelling plane waves are the diverging and converging cylindrical waves. Interpreting the equations $(2 \mathrm{a}),(2 \mathrm{~b})$ so that $\mathrm{A}^{ \pm}\left(\theta_{z}\right)$ are now the cylindrical wave amplitude components for the positive and negative cylindrical waves that have longitudinal propagation constants

$$
\beta=k_{2} \cos \theta_{z}
$$

We can then use these cylindrical wave reflection coefficients $R\left(\theta_{z}\right)$ to determine the radiation correction.

However, before we discuss these more involved calculations, let us consider the application of this correction to the volume Current Method for the simple situation of scattering from a plane wave incident from the more dense medium onto an interface between two homogeneous dielectric waveguides with a sinusoidally perturbed interface. As usual, we will consider a length, $2 \mathrm{~L}$, in the z-direction of the interface, which is perturbed and we consider the magnitude of the perturbation to be small compared to the wavelength. In the perturbed section of the interface, the surface is described by

$$
\mathbf{x}=a \sin \Omega z, \quad a<1
$$

as in fig. 3 , and the induced current density $\underset{\sim}{\sim}$ is

$$
\underset{\sim}{J}=-i \omega \Delta \varepsilon \underset{\sim}{\mathrm{E}} \operatorname{inc}
$$

where $\underset{\sim}{E}$ inc is the incident unperturbed at the surface. Considering a plane wave whose electric field is polarised perpendicular to the plane 


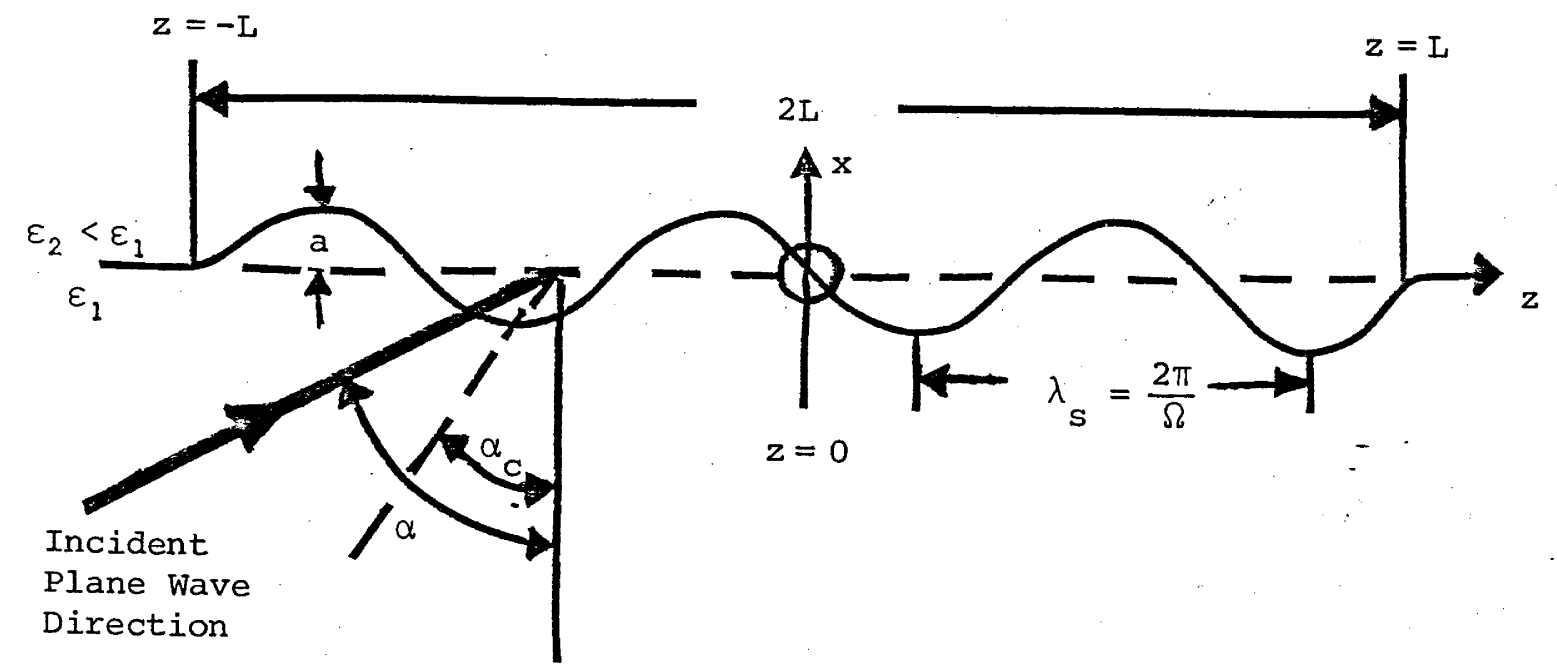

Fig. 3: A plane wave incident on a planar interface between two homogeneous dielectrics with a segment of interface perturbed by a sinusoidal fluctuation of amplitude $a<<\lambda$, the wavelength of the incident plane wave. 
of incidence, $\underset{\text { inc }}{E_{\text {inc }}}$ becomes

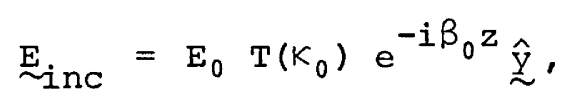

where the transmission coefficient for the plane wave is [11]

$$
T\left(K_{0}\right)=\frac{2 K_{0}}{K_{0}+i \gamma_{0}}
$$

with

$$
\begin{aligned}
& \kappa_{0}=\left(k_{1}^{2}-\beta_{0}^{2}\right)^{\frac{1}{2}} \\
& \gamma_{0}=\left(\beta_{0}^{2}-k_{2}^{2}\right)^{\frac{1}{2}}
\end{aligned}
$$

and $\mathrm{E}_{0}$ is the amplitude of the incident wave. Due to the symmetry of the radiation, from equations (2)

$$
A^{+}\left(\theta_{z}\right)=A^{-}\left(\theta_{z}\right)
$$

and the field will be polarised as the incident field so that

$$
A_{\text {tot }}^{+}\left(\theta_{z}\right)=A^{+}\left(\theta_{z}\right)\left(1+R_{2}\left(\theta_{z}\right)\right)
$$

Letting

$$
\begin{aligned}
& \rho^{2}=\left(k_{2}^{2}-\beta^{2}\right)=k_{2}^{2} \sin ^{2} \theta_{z} \geqslant 0 \\
& \sigma^{2}=\left(k_{1}^{2}-\beta^{2}\right) \geqslant 0,
\end{aligned}
$$

the Fresnel's reflection coefficient becomes [11]

$$
R_{2}\left(\theta_{z}\right)=\frac{\rho-\sigma}{\sigma+\rho}
$$

so that equation (8) becomes

$$
A_{\text {tot }}^{+}\left(\theta_{z}\right)=A^{+}\left(\theta_{z}\right)\left(\frac{2 \rho}{\rho+\sigma}\right)
$$

$\mathrm{A}^{+}\left(\theta_{z}\right)$ is the amplitude of the plane wave component of the radiation field for the radiation from the source in free space, i.e. the amplitude obtained by the Volume Current Method. Then, using equation (11) and the Volume Current Method, the total power scattered $P_{2}^{R}$ into 
the upper medium from an incident wave on the length, $2 \mathrm{~L}$, of the perturbed interface becomes (Appendix A)

$$
\mathrm{P}_{2}^{\mathrm{R}}=\left(\frac{\varepsilon_{2}}{\mu}\right)^{\frac{1}{2}} \frac{\mathrm{La}^{2}\left|\mathrm{E}_{0}\right|^{2} K_{0}^{2} \mathrm{k}_{1}^{2}\left(1-\frac{\varepsilon_{2}}{\varepsilon_{1}}\right)}{\mathrm{k}_{2}} \cdot \frac{\rho}{(\sigma+\rho)^{2}},
$$

where

and now we find

$$
\theta_{0}=\cos ^{-1}\left(\frac{\beta-\Omega}{k_{2}}\right)
$$

$$
\rho=k_{2} \sin \theta_{0}
$$

From a comparison of equation (12) with equation (4.3.17) of ref. 5, we can observe that this result agrees exactly with the transmission coefficient per unit length of perturbed interface obtained from ref. 5. This result can also be derived by an extension of coupled Mode Theory using the plane wave modes of the half spaces, and comparison with this result provides exact agreement once more [12].

The extension of this correction to the study of planar slab waveguides is straightforward except that one must now also include the transmission coefficient due to the surface irregularities of the opposite interface as well. In equation (2a) then, there is an extra term so that we must write, for a slab waveguide as in fig. 4

$x \geqslant d \quad A_{\text {tot }}^{+}\left(\theta_{z}\right)=A_{2}^{+}\left(\theta_{z}\right)+R_{2}^{S}\left(\theta_{z}\right) A_{2}^{-}\left(\theta_{z}\right)+T_{3}^{S}\left(\theta_{z}\right) A_{3}^{+}\left(\theta_{z}\right)$

and

$x \leqslant-d \quad A_{\operatorname{tot}}^{-}\left(\theta_{z}\right)=A_{3}^{-}\left(\theta_{z}\right)+R_{3}^{S}\left(\theta_{z}\right) A_{3}^{+}\left(\theta_{z}\right)+T_{2}^{S}\left(\theta_{z}\right) A_{2}^{-}\left(\theta_{z}\right)$,

where for generality we have assumed that the two cladding dielectrics are different. The reflection and transmission coefficients for plane waves incident from the cladding on the slab waveguide can be simply related to the local Fresnel's reflection and transmission coefficient for the two interfaces [13]. For the case of an incident even TE mode of a symmetric slab waveguide the power attenuation of the mode becomes, upon utilising the correction as in equation (15), for an amplitude, $2 \Delta \mathrm{d} \sin \Omega \mathrm{z}$, of the diameter fluctuation

$$
\alpha=\frac{\mathrm{P}^{\mathrm{R}}}{2 \mathrm{~L} \cdot \mathrm{P}_{0}}
$$




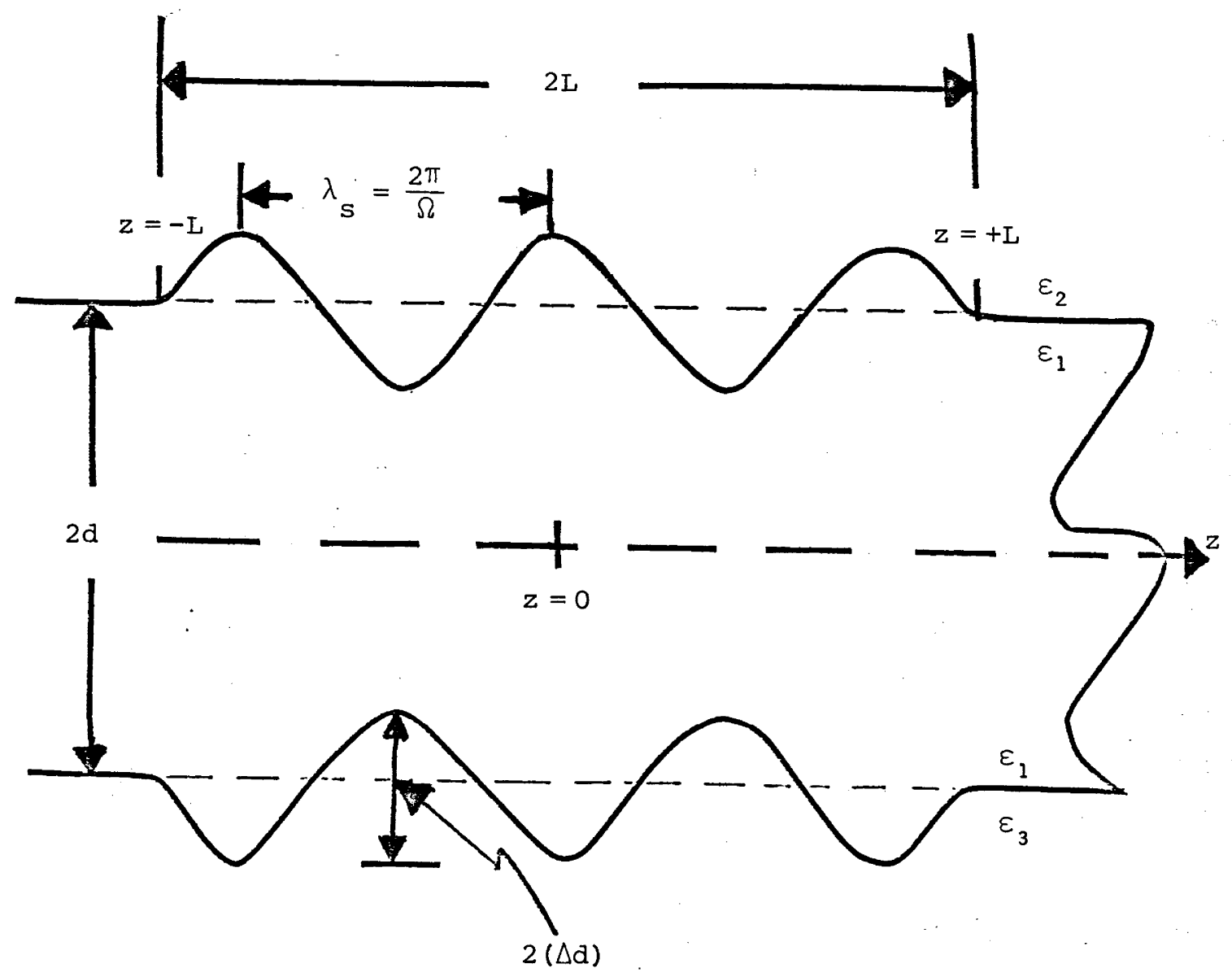

Fig. 4: The asymmetric slab waveguide with sinusoidal fluctuations of the width of the guide with a spatial wavelength of the perturbation $\lambda_{S}=2 \pi / \Omega$. 
where $P_{0}$ is the power in the mode incident on the length $2 \mathrm{~L}$ of the perturbed waveguide. $\mathrm{P}^{\mathrm{R}}$ is the total radiated power then (Appendix $\mathrm{B}$ )

$$
\alpha=\frac{(\Delta d)^{2} \cdot U_{0}^{2} W_{0} v^{2}}{2\left(\beta_{0} d^{4}\right)\left(1+W_{0}\right)} \cdot \frac{Q_{r} \cos ^{2} U_{r}}{\left(Q_{r}^{2} \cos ^{2} U_{r}+U_{r}^{2} \sin ^{2} U_{r}\right)},
$$

where the subscript $r$ refers to the radiation such that

$$
Q_{r}=k_{2} d \sin \theta_{0}
$$

where

$$
\begin{aligned}
& \theta_{0}=\cos ^{-1}\left(\frac{\beta_{0}-\Omega}{k_{2}}\right) \\
& U_{r}=\left(V^{2}+Q_{r}^{2}\right)^{\frac{1}{2}}
\end{aligned}
$$

with the same restrictions as to the range of spatial frequencies as in equation (13).

Comparing this result and equation (9.3-19) of ref. 11, we see that using the above corrections we have once again obtained perfect agreement for all spatial frequencies of the perturbation.

Now that we have displayed the utility of the correction to the Volume Current Method, let us consider the more practically significant situation of the radiation losses of the optical fibre of circular cross section.

\subsection{CORRECTION TO THE VOLUME CURRENT METHOD FOR CYLINDRICAL WAVEGUIDES}

In the last section we demonstrated how the elementary introduction of planar reflection and transmission coefficients for plane waves could be utilised to construct exact solutions for the radiative losses (correct to first order in the perturbation) from the analysis of the Volume Current Method. We briefly discussed the extension of this principle to the radiative losses in cylindrical waveguides. If such an analysis is to be utilised in cylindrical structures, electromagnetic cylindrical wave equivalents of plane wave reflection and 
transmission coefficients must be developed. To the author's knowledge, no such coefficients have been reported in the literature for the threedimensional cylindrical wave. In the following section, we shall develop such a cylindrical reflection coefficient and apply it to the analysis of the step-index dielectric optical fibre with sinusoidal variations in the radius.

\subsubsection{The Cylinarical Electromagnetic Wave Reflection Coefficient}

The study of non-planar equivalents to the Fresnel reflection and transmission coefficients does not appear to have been successfully treated. Wait [15] and snyder [16] in their respective analyses of the continuous mode spectrum of uniform dielectric cylinders embedded in an infinite uniform cladding, developed the "incident transverse electric" (ITE) and "incident transverse magnetic" (ITM) modal representation from the consideration of the plane wave scattering from the cylinder. However, such modes cannot be considered to provide the equivalent description to the TE and TM plane wave modes of planar geometries, since one cannot decompose each ITE and ITM mode into cylindrical fields of the same structural form, i.e. convergent and divergent cylindrical electromagnetic waves with the same inter-relationships between the magnitudes of the electromagnetic wave components, for both waves.

In the cylindrical representation, the waves analogous to the plane waves travelling toward the positive and negative infinities are the Hankel functions [17]. However, the cylindrical waves travel radially and are either converging towards or diverging from the axis of the cylindrical system, depending on the "kind" of the Hankel function. From the asymptotic representation of the Hankel functions for large arguments [17], the Hankel function of the first kind, $\mathrm{H}_{\ell}^{(1)}$, can be recognised as the converging wave, and the Hankel function of the second kind, $\mathrm{H}_{\ell}^{(2)}$, the diverging wave, for exp\{iwt\} time variations.

For an electromagnetic wave to be reflected and retain its "integrity", the relationships between the individual electromagnetic field components of the wave must remain unchanged upon reflection. In 
the cylindrical wave, all field components can be determined from the longitudinal (i.e. parallel to the axis) electric and magnetic fields. Thus, the integrity of the cylindrical wave under reflection will be preserved if the ratio of the longitudinal components of the field remains unchanged.

If we consider an incident converging cylindrical electromagnetic wave with longitudinal electric and magnetic field amplitudes $C_{l}^{i}$ and $D_{l}^{i}$ respectively, for reflection from a concentric cylinder under which the cylindrical wave integrity is preserved, we require that the longitudinal electric and magnetic field amplitudes of the reflected wave $C_{l}^{r}$ and $D_{l}^{r}$ are related to the incident longitudinal fields via

and

$$
\left.\begin{array}{l}
C_{l}^{r}=R C_{l}^{i} \\
D_{l}^{r}=R D_{l}^{i}
\end{array}\right\}
$$

so that with equation (1) satisfied, the convergent cylindrical wave would be reflected from the concentric cylindrical surface with an amplitude reflection coefficient $R$.

Matching the fields, generated by these longitudinal electric and magnetic fields, across the boundary surface of the cylinder of arbitrary dielectric profile of radius, $\rho$, where the longitudinal fields are, ${ }^{\dagger}$ neglecting the common factor $\exp \{-i(\ell \phi+\beta z)\}$,

$$
\left.\begin{array}{rlrl}
E_{z}=A_{\ell} f_{\ell}(r) & : & r \leqslant \rho \\
& =C_{\ell}^{i} H_{\ell}^{(1)}\left(\frac{W r}{\rho}\right)+C_{\ell}^{r} H_{\ell}^{(2)}\left(\frac{Q r}{\rho}\right) & : & r \geqslant \rho
\end{array}\right\}
$$

and

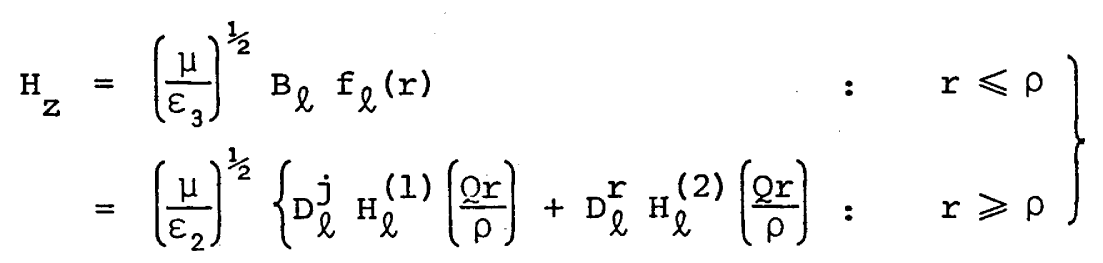

where $\varepsilon_{3}$ is the dielectric permittivity of the core region just inside

$+$

We have used the arbitrary dielectric profile of Chapter 2 to demonstrate that the subsequent results are entirely general, where $f_{\ell}(r)$ is the solution of the cylindrical wave equation, equation $(2.1 .27)$. 
the boundary at $r=\rho, \varepsilon_{2}$ is the cladding dielectric permittivity, and

$$
Q^{2}=\left(k_{2}^{2}-\beta^{2}\right) \cdot \rho^{2} \geqslant 0,
$$

where $B$ is the longitudinal propagation constant of the incident wave along the axis of the cylinder, and using equations (2A.1) - (2A.4) for the transverse field components, and equations (1) above, provides six equations in six unknowns, so that we can uniquely determine the interrelationship between the longitudinal electric and magnetic field magnitudes that allow for a reflection coefficient of a cylindrical wave from a cylinder as described above by equation ( 1 ).

For weakly guiding dielectric optical waveguides, we find that this inter-relationship between the amplitudes of the incident cylindrical wave longitudinal field components is (Appendix C)

$$
D_{\ell}= \pm i C_{\ell}^{i}
$$

for which the reflection coefficient defined in equation (1) becomes

$$
\left.R=-\frac{\left(\eta(1) \mp K \mathrm{H}_{\ell}^{(1)}\right.}{\eta(2) \mp \eta \mathrm{H}_{\ell}^{(2)}}\right)
$$

where the - and + signs are for the two solutions of equation (5) respectively, where

and

$$
n(k)=\left(\frac{H_{l}^{(k)}(Q) f_{l}^{\prime}(\rho)}{U^{2}(\rho) f_{\ell}(\rho)}-\frac{H_{l}^{(k)^{\prime}}(Q)}{Q^{2}}\right)
$$

$$
K=\left(\frac{\beta l}{k_{2} \rho}\right)\left(\frac{V^{2}(\rho)}{U^{2}(\rho) \cdot Q^{2}}\right)
$$

where $V^{2}(\rho)$ is defined in equation $(2.2 .5)$ and

$$
U^{2}(\rho)=V^{2}(\rho)+Q^{2}
$$

The ' in equation (7) indicates $\partial / \partial r$, not differentiation with respect to the argument. 
From a comparison of equation (5) with equation (2.2.28) for the inter-relationship between the longitudinal field components of the bound modes, we find that equation (5) is identical to equation (2.2.28). This result is not as surprising as one first suspects since the modal electromagnetic fields must retain their integrity as they propagate along the cylindrical waveguide. Thus, to retain the nomenclature of the bound mode waves, we shall call the cylindrical waves with the upper - sign of equation (5) the $\mathrm{HE}_{\ell}$ wave and the wave with the lower + sign the $\mathrm{EH}_{\&}$ wave.

Since the combination, defined in equation (5), of the $\mathrm{TE}_{\ell}$ and $\mathrm{TM}_{\ell}$ components of the cylindrical wave are orthogonal and the $\mathrm{TE}_{\ell}$ and ${ }^{\mathrm{TM}} \ell$ waves are orthogonal [18], these electromagnetic cylindrical waves form an orthogonal set of fields in the cladding.

For the step-index waveguide, equation (7) becomes

where now

$$
\eta(k)=\frac{I}{\rho}\left(\frac{H_{\ell}^{(k)}(Q) J_{\ell}^{\prime}(U)}{U J_{\ell}(U)}-\frac{H_{\ell}^{(k)}(Q)}{Q}\right) \text {, }
$$

$$
U^{2}=\rho^{2}\left(k_{1}^{2}-\beta^{2}\right)
$$

and now the ' refers to differentiation with respect to the argument.

$$
\text { From an inspection of equation (6) for the reflection }
$$

coefficient, we note that as $Q$ tends to zero, $\mathrm{H}_{\ell}^{(\mathrm{k})}$ tends to infinity and the reflection coefficient becomes $(-1)$ similarly to the plane wave reflection coefficient at grazing incidence [11], since

$$
Q=k_{2} \rho \sin \theta_{z}^{(2)}
$$

where $\theta_{z}^{(2)}$ is the angle the component rays of the wave make with the $z$-axis in the cladding. We are now in a position to discuss the volume Current Method correction for cylindrical waveguides. 


\subsubsection{Volume Current Method Correction for Cylindrical Waveguides}

As discussed in the planar situation, the radiation from sources can be considered by investigating the individual plane wave components of the radiation field. In cylindrical guides this decomposition into the cylindrical wave components can be treated by Fourier-Bessel integrals [19]. Thus, we can confine ourselves to one cylindrical wave and discuss the effect of the re-introduction of the core of the optical fibre into the volume Current Method calculations.

To use the cylindrical reflection coefficient above for the cylindrical electromagnetic waves impinging on a concentric dielectric cylinder, we must first appreciate the radiation process occurring in a cylindrical structure. Considering one component cylindrical wave of the radiation with angle $\theta_{z}^{(2)}$ to the axis in the cladding from a cylindrical radiator of radius $\rho_{r}$ in free space, as drawn in cross section in fig. 5a, the radiation in the two regions, i.e. inside the radiator $r \leqslant \rho_{r}$ and outside $r \geqslant \rho_{r}$, the radiation fields can be derived from the longitudinal components with the form [20] for the spatial variation, defined by

and

$$
\left.\begin{array}{rlr}
\psi(r) & =J_{\ell}\left(k_{2} \rho_{r} \sin \theta_{z}^{(2)}\right) \mathrm{H}_{\ell}^{(2)}\left(k_{2} r \sin \theta_{z}\right) & \text { for } r \geqslant \rho_{r} \\
& =\mathrm{H}_{\ell}^{(2)}\left(k_{2} \rho_{r} \sin \theta_{z}^{(2)}\right) J_{\ell}\left(k_{2} r \sin \theta_{z}^{(2)}\right) & \text { for } r \leqslant \rho_{r}
\end{array}\right\},
$$

where $\mathrm{J}_{\ell}\left(\mathrm{k}_{2} \rho_{r} \sin \theta_{\mathrm{z}}^{(2)}\right)$ and $\mathrm{H}_{\ell}^{(2)}\left(\mathrm{k}_{2} \rho_{r} \sin \theta_{z}\right)$ can be considered as the strength of the radiation field due to the radiator.

Writing equations (13) in terms of the component Hankel functions, we have [17]

$$
\begin{aligned}
2 \psi(r)= & \mathrm{H}_{\ell}^{(1)}\left(\mathrm{k}_{2} \rho_{r} \sin \theta_{z}^{(2)}\right) \mathrm{H}_{\ell}^{(2)}\left(\mathrm{k}_{2} r \sin \theta_{z}^{(2)}\right) \\
& +\mathrm{H}_{\ell}^{(2)}\left(\mathrm{k}_{2} \rho_{r} \sin \theta_{z}^{(2)}\right) \mathrm{H}_{\ell}^{(2)}\left(\mathrm{k}_{2} r \sin \theta_{z}^{(2)}\right) \text { for } r \geqslant \rho_{r}
\end{aligned}
$$

and 


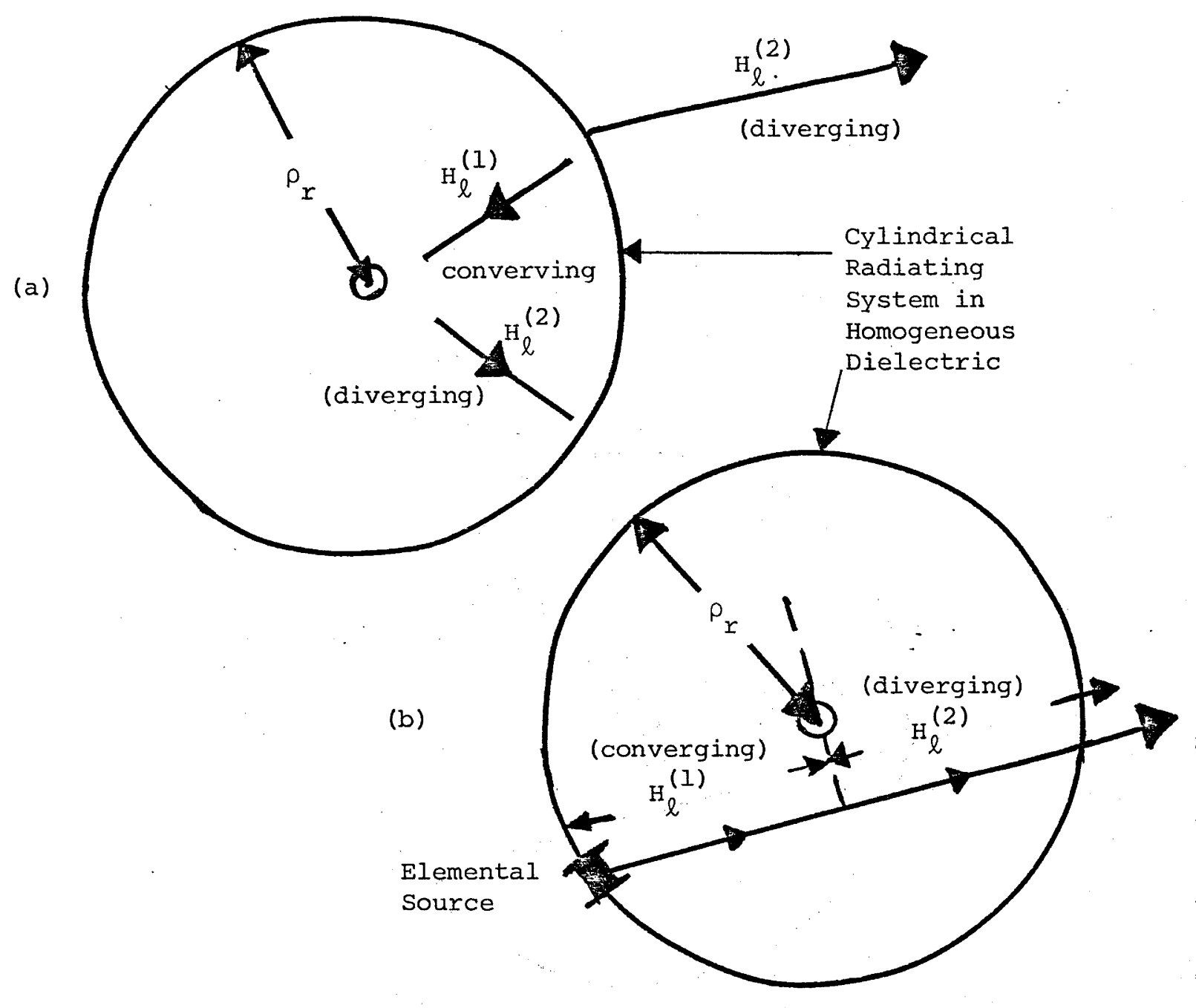

Fig. 5: (a) A decomposition of the radiation from a cylindrical radiator of radius $\rho_{r}$ into its component convergent and divergent cylindrical waves.

(b) The radiation from one elemental source of the cylindrical radiator, displaying the dual nature of the radiation emanating from this source, depending on the distance from the source. 


$$
\begin{aligned}
2 \psi(r)= & \mathrm{H}_{\ell}^{(2)}\left(k_{2} \rho_{r} \sin \theta_{z}^{(2)}\right) \mathrm{H}_{\ell}^{(1)}\left(k_{2} r \sin \theta_{z}^{(2)}\right) \\
& +\mathrm{H}_{\ell}^{(2)}\left(k_{2} \rho_{r} \sin \theta_{z}^{(2)}\right) \mathrm{H}_{\ell}^{(2)}\left(k_{2} r \sin \theta_{z}^{(2)}\right) \text { for } r \leqslant \rho_{r} .
\end{aligned}
$$

From equations (14), we see that for $r \leqslant \rho_{r}$, the radiation is composed of both a converging and a diverging cylindrical wave. To use the cylindrical reflection coefficients we must understand how these two waves occur. From fig. 5b, radiation emanating for the cylinder into the inner regions $r \leqslant \rho_{r}$ has both the properties of the cylindrically convergent and divergent wave depending on the distance away from its source. Therefore, we can now see that the contribution, $\mathrm{H}_{\ell}^{(2)}\left(k_{2} \rho_{r} \sin \theta_{z}\right) \mathrm{H}_{\ell}^{(2)}\left(k_{2} r \sin \theta_{z}\right)$, to the radiation for $r \geqslant \rho_{r}$ arises from the contribution to the radiation that initially travels inside the radiator. Thus, in the presence of another cylindrical boundary of radius $\rho<\rho_{r}$ within the radiating cylinder, this contribution to the radiation at infinity does not occur. However, the cylindrical convergent wave now reflects from this boundary to provide an additional divergent wave at infinity, so that the amplitude of the divergent wave outside the radiators should now be written as

$$
\begin{aligned}
& 2 \psi^{\text {tot }}(r)=\mathrm{H}_{\ell}^{(2)}\left(\mathrm{k}_{2} r \sin \theta_{t}^{2}\right)\left(\mathrm{H}_{\ell}^{(1)}\left(\mathrm{k}_{2} \rho_{r} \sin \theta_{\mathrm{z}}^{(2)}\right)\right. \\
&\left.+\mathrm{R}(\rho) \mathrm{H}_{\ell}^{(2)}\left(\mathrm{k}_{2} \rho_{\mathrm{r}} \sin \theta_{\mathrm{z}}^{(2)}\right)\right),
\end{aligned}
$$

where $R(\rho)$ is the reflection coefficient from the cylinder of radius $\rho$.

From a comparison of equation (15) and equation (13), we see that to correct for the effect of the cylinder within the radiating sources we replace the excitation strength of the divergent wave, $J_{\ell}\left(k_{2} \rho_{r} \sin \theta_{z}\right)$, by the coefficient of the divergent wave defined by equation (15), i.e.

$$
2 J_{\ell}\left(k_{2} \rho_{r} \sin \theta_{z}^{(2)}\right) \rightarrow H_{\ell}^{(1)}\left(k_{2} \rho_{r} \sin \theta_{z}^{(2)}\right)+R(\rho) H_{\ell}^{(2)}\left(k_{2} \rho_{r} \sin \theta_{z}^{(2)}\right) \text {. }
$$

If we now consider the application of this correction for the case discussed in Chapter 3 of a step-index fibre with sinusoidal variations in the radius of the fibre, by replacing all terms $\mathrm{J}_{\mathrm{m}}\left(\mathrm{k}_{2} \rho \sin \theta_{0}\right)$ by the terms defined in equation $(16)$, we obtain the 
corrected result for the modal power attenuation. From the symmetry of the radiation loss process, we would expect the radiation to remain in the same polarisation as the incident modal field. Thus for the radiation from the $\mathrm{HE}_{11}$ mode, we choose $\mathrm{R}$ defined by equation (6) for the upper "-" sign for the radiation sources coincident with the corecladding interface, so that using equation (16) in equation (3.3.38), we have (Appendix D)

$$
\alpha=\frac{a^{2} \cdot w_{0}^{2} \cdot V^{2} \Psi\left(U_{r} Q_{r}\right)}{2 \pi k_{2} \rho^{2} \cdot J_{1}^{2}\left(U_{0}\right) \cdot\left(\eta(2)-\kappa H_{1}^{(2)}\left(Q_{r}\right)\right)^{2} J_{1}^{2}\left(U_{r}\right)\left(Q_{r} U_{r}\right)^{2}},
$$

where

where

$$
\begin{aligned}
\Psi\left(U_{r} Q_{r}\right)= & J_{0}^{2}\left(U_{0}\right)\left\{J_{1}^{\prime}\left(U_{r}\right)+\frac{\beta_{r} \ell}{k_{2} U_{r}} J_{\ell}\left(U_{r}\right)+\frac{1}{\rho^{2} k_{2}\left(\beta_{r}+k_{2}\right)}\right\}^{2} \\
+ & {\left[\frac{\beta_{r}}{k_{2}} J_{0}\left(U_{0}\right)\left(J_{\ell}^{\prime}\left(U_{r}\right)+\frac{\beta_{r} \ell}{k_{2} U_{r}} J_{\ell}\left(U_{r}\right)+\frac{1}{k_{2} \rho^{2}\left(\beta_{r}+k_{2}\right)}\right)\right.} \\
& \left.+\frac{U_{0}}{k_{2} \beta_{0} \rho^{2}} Q_{r} J_{1}\left(U_{0}\right) J_{1}\left(U_{r}\right)\right]^{2},
\end{aligned}
$$

and

$$
Q_{r}=k_{2} \rho \sin \theta_{0}=\rho\left(k_{2}^{2}-\beta_{r}^{2}\right)^{\frac{1}{2}}
$$

$$
\mathrm{u}_{r}^{2}=\mathrm{v}^{2}+\mathrm{Q}_{r}^{2}
$$

are the transverse wavenumbers of the core and cladding for the radiation, and $\mathrm{U}_{0}, \mathrm{~W}_{0}, \beta_{0}$ refer to the incident $\mathrm{HE}_{11}$ mode.

For paraxially directed radiation, i.e.

$$
\frac{\beta_{r}}{k_{2}} \sim \cos \theta_{0} \sim 1
$$

Using the recurrence relations for Bessel functions [17] and equation (21), the power attenuation becomes

$$
\alpha=\frac{a^{2} \cdot w_{0}^{2} \cdot v^{2}}{2 \pi k_{2} \rho^{2} J_{1}^{2}\left(U_{0}\right)} \frac{J_{0}^{2}\left(U_{0}\right) \cdot J_{0}^{2}\left(U_{r}\right)\left(I+\cos ^{2} \theta_{0}\right)}{\left|Q_{r} H_{1}^{(2)}\left(Q_{r}\right) J_{0}\left(U_{r}\right)-U_{r} J_{1}\left(U_{r}\right) H_{0}^{(2)}\left(Q_{r}\right)\right|^{2}},
$$

where 
and

$$
Q_{r}=k_{2} \rho \sin \theta_{0}
$$

and

$$
\left.\begin{array}{l}
\theta_{0}=\cos ^{-1}\left(\frac{\beta_{0}-\Omega}{k_{2}}\right) \\
U_{r}=\left(v^{2}+k_{2}^{2} \rho^{2} \sin ^{2} \theta_{0}\right)^{\frac{1}{2}}
\end{array}\right\} .
$$

Equation (22) agrees exactly with the result calculated by Coupled Mode Theory [21]. Due to the complexity of the radiation modes of the weakly guiding optical waveguide, seldom are they ever used except with the paraxial ray approximation. However, by the use of this reflection technique to correct the Volume Current Method, we have now derived a result that is correct for the radiation at all angles of the radiation ${ }^{\dagger}$ without much additional analysis required. In comparison to the exact analysis of the Coupled Mode treatment, in which orthogonalisation of the radiation modes is required, this correction technique is extremely simple.

Thus, we have developed a correction technique that widens the range of the applicability of the Volume Current Method to all radiation mechanisms in dielectric optical waveguides. In fig. 6, we plot the ratio of equation $(22), \alpha / \alpha_{\max }\left(Q_{r} \rightarrow U_{r}\right)$, and the weakly guiding waveguide correction of the Volume Current Method postulated by Marcuse [5] for the $V=2.4$ fibre of fig. 1 .

We can see that in the forward direction, although the axial radiation is correct, a reasonably large discrepancy occurs for nearly forward-directed radiation.

\subsection{DISCUSSION}

We have demonstrated a technique for correcting the facile computations of the Volume Current Method that does not involve arduous and often tedious analysis, since by the Volume Current Method, the radiation from the irregularities is calculated and the correction

\footnotetext{
+ In the derivation of the reflection coefficient, we did not need to assume the paraxial approximation.
} 


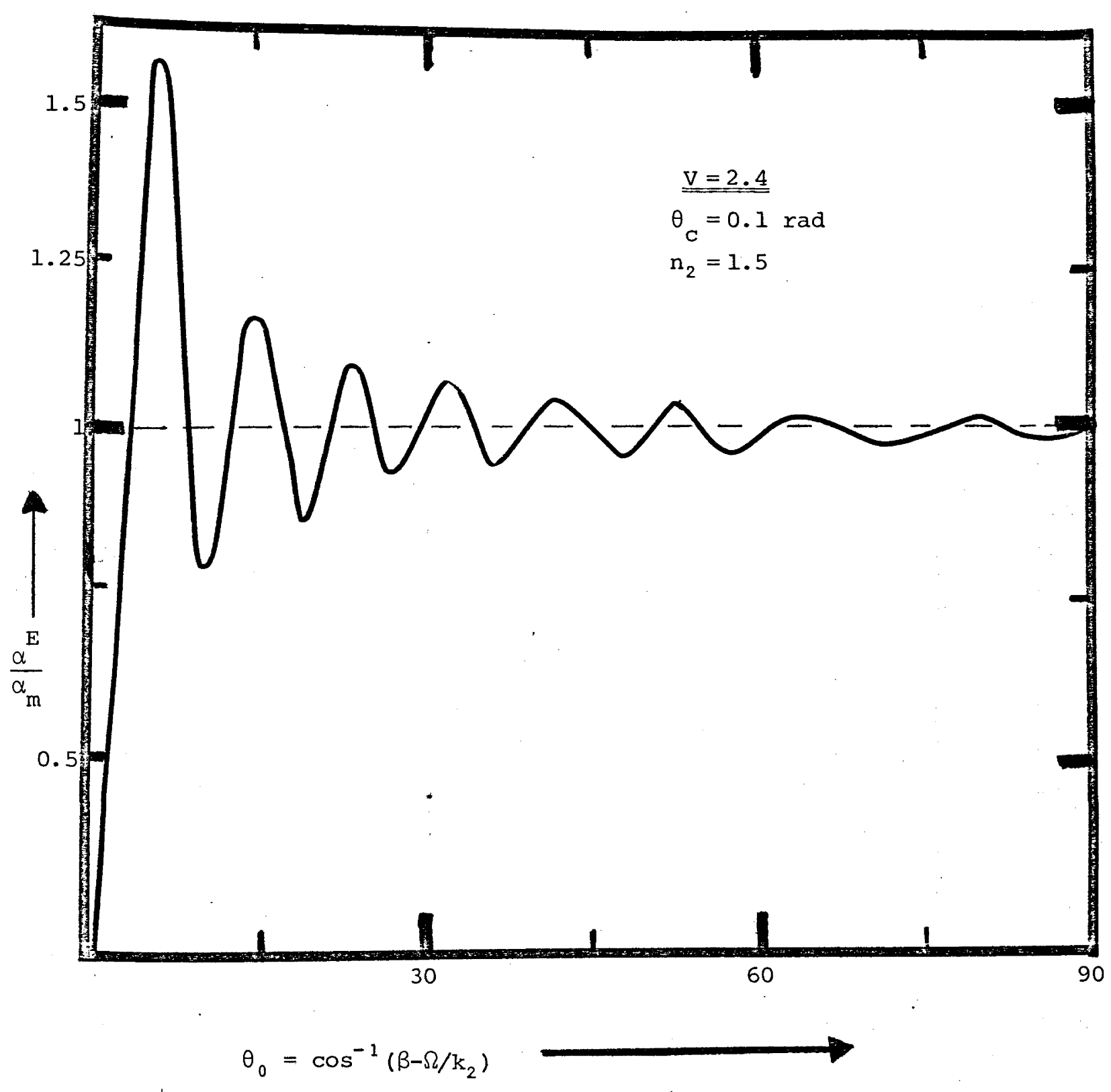

Fig. 6: Ratio of the exact modal power attenuation coefficient, $\alpha^{E}$, to the modal power attenuation coefficient derived by Marcuse using the Volume Current Method plus Marcuse's correction, $\alpha_{m}$, for a $v=2.4$ step-index fibre with an acceptance angle $\theta_{c}=0.1 \mathrm{rad}$, for different spatial frequencies of the sinusoidal radius perturbation, $\Omega$. 
simply accounts for the presence of the core of the waveguide. By this analysis, we have isolated all the physical processes occurring for radiators in the presence of a dielectric boundary. Equation (3.17) is the first result ever presented that is accurate over the entire range of the radiation. This correction circumvents the necessity of Coupled Mode treatments while retaining the elegant simplicity and physical insight of the volume Current Method. From the above discussion we can see that it also allows for an immediate correction for finitely clad waveguides, by application of the reflection and transmission coefficients at the outer core-cladding boundary.

Although the above analysis has been restricted to step-index waveguides, we can see that the analysis can be readily adjusted to accommodate graded-index waveguides, as we have already done for the cylindrical wave reflection coefficient derived in section 3 . 


\section{APPENDIX A \\ RADIATION SCATTERED FROM AN INCIDENT PLANE WAVE DUE TO A SINUSOIDAL PERTURBATION OF THE INTERFACE BETWEEN TWO HOMOGENEOUS DIELECTRICS}

In this appendix, we shall consider the scattering of an incident $\mathrm{TE}$ plane wave on the interface described in fig. 3.

The electric field at the interface is the electric field at the unperturbed interface, and since the perturbation is slight, we assume the field over the transverse extent of the irregularity is constant

$$
E_{Y}=T\left(K_{0}\right) E_{0} e^{-i \beta_{0} z}
$$

where $T\left(K_{0}\right)$ is the transmission coefficient of the incident plane wave and is defined to be

where

$$
T\left(K_{0}\right)=\frac{2 \kappa_{0}}{\kappa_{0}+i \gamma_{0}},
$$

$$
\left.\begin{array}{l}
k_{0}^{2}=k_{1}^{2}-\beta_{0}^{2} \geqslant 0 \\
\gamma_{0}^{2}=\beta_{0}^{2}-k_{2}^{2} \geqslant 0
\end{array}\right\} .
$$

Using the two-dimensional form of $\mathrm{S}^{\mathrm{R}}$ obtained by multiplying equation $(3.1 .20)$ by $\left(2 \pi R / k_{2}\right)^{\frac{1}{2}}[14]$, we have by the volume Current Method the time averaged radiant energy flux in $\varepsilon_{2}$ is

$$
S^{R}=\left(\frac{\mu}{\varepsilon_{2}}\right)^{\frac{1}{2}} \frac{k_{2}}{16 \pi R}\left|\underset{\sim}{\stackrel{R}{R}} \times \int_{V^{\prime}} \underset{\sim}{J} \exp \left\{i k_{2} \underset{\sim}{\stackrel{R}{R}} \cdot \underset{\sim}{R^{\prime}}\right\} d V^{\prime}\right|^{2},
$$

where

$$
\underset{\sim}{J}=i \omega\left(\varepsilon_{1}-\varepsilon_{2}\right) \text { a T }\left(\kappa_{0}\right) \cdot E_{0} \sin \Omega z e^{-i \beta_{0} z} \hat{\underline{z}}
$$

and 


$$
\hat{\underline{R}}=\sin \theta_{\mathbf{z}} \underset{\mathrm{x}}{\sim}+\cos \theta_{\mathbf{z}} \underset{z}{\stackrel{\hat{z}}{\sim}} \text {. }
$$

Then equation (4) becomes, for the energy flux in the $\theta_{z}$ direction

$$
S^{R}=\left(\frac{\mu}{\varepsilon_{2}}\right)^{\frac{1}{2}} \frac{k_{2}}{16 \pi R}\left(\Delta \varepsilon \cdot \omega a E_{0} T\left(k_{0}\right)\right)^{2}\left\{\frac{\sin k_{2} \gamma_{+} L}{k_{2} \gamma_{+}}+\frac{\sin k_{2} \gamma_{-} L}{k_{2} \gamma_{-}}\right\}^{2},
$$

where

$$
k_{2} \gamma_{ \pm}=k_{2} \cos \theta_{z}-\beta \pm \dot{\Omega}
$$

From the symmetry of equation (7) in the $\pm \theta_{z}$ directions, the radiated fields satisfy

$$
S^{R}=\left|A^{ \pm}\left(\theta_{z}\right)\right|^{2}
$$

Thus the time averaged Poynting vector $s_{\text {tot }}^{R}$ for the corrected radiation to include the presence of the dielectric interface, from equation (2.2a) and equation (9) becomes

$$
S_{\text {tot }}^{R}=s^{R}\left|1+R_{2}\left(\theta_{z}\right)\right|^{2} \text {, }
$$

where $S^{R}$ is defined in equation (9) and

and

$$
\left.\begin{array}{rl}
\rho^{2} & =\left(k_{2}^{2}-k_{2}^{2} \cos ^{2} \theta_{z}\right)=k_{2}^{2} \sin ^{2} \theta_{z} \\
\sigma & =\left(k_{1}^{2}-\beta_{r}^{2}\right)^{\frac{1}{2}},
\end{array}\right\}
$$

where

$$
\beta_{r}=k_{2} \cos \theta_{z}
$$

we find [11]

$$
R_{2}\left(\theta_{z}\right)=\left(\frac{\rho-\sigma}{\rho+\sigma}\right)
$$

so that equation (10) becomes

$$
S_{\text {tot }}^{R}=\frac{4 \rho^{2}}{(\rho+\sigma)^{2}} S^{R}
$$

Thus, using the integral results of Appendix 3.C equation (3C.11) for the integral over $\theta_{z}$, the total power radiated into the upper half space $\mathrm{P}_{2}^{\mathrm{R}}$ becomes 


$$
P^{R}=R \int_{0}^{\pi} S_{\text {tot }}^{R} d \theta
$$

which from equations (7) and (12) for a length 2I of perturbed interface is

$$
\mathrm{P}^{\mathrm{R}}=\frac{\mathrm{La}^{2} \cdot K_{0}^{2} \cdot k_{1}^{2}\left(1-\frac{\varepsilon_{2}}{\varepsilon_{1}}\right)}{\omega \mu} \frac{\rho}{(\rho+\sigma)^{2}}
$$

for spatial frequencies

$$
\beta-k_{2} \leqslant \Omega \leqslant \beta+k_{2}
$$

and

$$
\rho=k_{2} \sin \theta_{0},
$$

where

$$
\theta_{0}=\cos ^{-1}\left(\frac{\beta-\Omega}{k_{2}}\right) \text {. }
$$




\section{APPENDIX B \\ RADIATION FROM THE EVEN TE MODES OF A SYMMETRIC SLAB WAVEGUIDE DUE TO DIAMETER FLUCTUATIONS}

The electric field of the symmetric TE mode of symetric slab waveguide carrying unit guided power is [11]

where

$$
E_{y}=A_{e} \cos u e^{-i \beta_{0} z} \quad \text { at } x= \pm d \text {, }
$$

$$
A_{e}=\left(\frac{2 \omega \mu w_{0}}{B d(1+W)}\right)^{\frac{1}{2}} \text {, }
$$

where the slab waveguide has a width $2 d$.

Now from the discussion of section 2, we need only calculate the radiated power in the position $\mathrm{x}$-direction by the Volume Current Method, as in fig. 4, where the amplitude of the width fluctuation is $(2(\Delta d) \sin \Omega z)$.

From equation (A.4), the time averaged Poynting vector of the radiation for the fluctuation is

$$
S^{R}=\left(\frac{\mu}{\varepsilon_{2}}\right)^{\frac{1}{2}} \frac{k_{2}}{16 \pi R}\left(\Delta \varepsilon \cdot \omega \cdot \Delta d \cdot A_{e}\right)^{2}\left\{\frac{\sin k_{2} \gamma_{+} L}{k_{2} \gamma_{+}}+\frac{\sin k_{2} \gamma_{-} L}{k_{2} \gamma_{-}}\right\}^{2},
$$

where $\gamma_{ \pm}$are defined in equation (A.8).

Once again the symmetry of the slab structure allows us to re-write equation $(2.16)$ as

$$
S_{\text {tot }}^{R}\left(\theta_{z}\right)=S_{R}\left(\theta_{z}\right)\left(1+T^{S}\left(\theta_{z}\right)+R^{S}\left(\theta_{z}\right)\right),
$$

where $T^{S}$ and $R^{S}$ are the amplitude transmission and reflection coefficients for the plane wave incident on the slab from the cladding 
at angle $\theta_{z}$ to the interface. We can write [13]

$$
1+R\left(\theta_{z}\right)+T\left(\theta_{z}\right)=\frac{2 Q_{r} \cos U_{r}}{\left(a_{r} \cos U_{r}+i U_{r} \sin U_{r}\right)}
$$

where

and

$$
\left.\begin{array}{l}
Q_{r}=\mathrm{k}_{2} \rho \cdot \sin \theta_{z} \\
U_{r}=\left(v^{2}+Q_{r}^{2}\right)^{\frac{1}{2}}
\end{array}\right\}
$$

with $\mathrm{V}$ the usual normalised waveguide frequency.

Then, using equations (4) and (5) in the evaluation of the total radiated power, we have

$$
P_{\text {tot }}^{R}=R \cdot \int_{0}^{2 \pi} S_{\text {tot }}^{R} d \theta_{z}
$$

and with the usual evaluation of the $\theta_{z}$ integral as in Appendix $3 C$, the power attenuation coefficient becomes

$$
\alpha=\frac{P_{\text {tot }}^{R}}{2 L}
$$

since $A_{e}$ was chosen for united guided modal power, and

$$
\alpha=\frac{(\Delta d)^{2}}{2} \frac{U_{0}^{2} W_{0} v^{2}}{\left(B d^{4}\right)(I+W)} \cdot \frac{Q_{r} \cos ^{2} U_{r}}{\left(Q_{r}^{2} \cos ^{2} U_{r}+U_{r}^{2} \sin ^{2} U_{r}\right)}
$$

where

and

$$
\left.\begin{array}{l}
Q_{r}=k_{2} \mathrm{~d} \sin \theta_{0} \\
\theta_{0}=\cos ^{-1}\left(\frac{\beta-\Omega}{k_{2}}\right)
\end{array}\right\} .
$$

This is in exact agreement with the evaluation of the result by the exact Coupled Mode Theory equation (9.3.19) of ref. 11. 


\begin{abstract}
APPENDIX C
THE CYLINDRICAL ELECTROMAGNETIC WAVE REFLECTION COEFFICIENT OF A CONVERGENT WAVE INCIDENT ON A CONCENTRIC CYLINDER OF ARBITRARY DIELECTRIC PROFILE
\end{abstract}

In this appendix, we will determine the combination of $\mathrm{TE}$ and TM components of a wave with azimuthal variation, $\exp \{-i \ell \phi\}, l \geqslant 0$, converging on a cylindrical surface whose inter-relationships between electromagnetic field components is preserved upon the reflection with a subsequent reflection coefficient $R$ in a fashion analogous to the reflection coefficient of plane waves.

For a harmonic time dependence of the form exp\{iwt\}, the longitudinal field components can be written as (ignoring the common factor $\exp \{i(\omega t-\beta z-\ell \phi)\})$

and

$$
\begin{aligned}
\mathrm{E}_{z} & =\mathrm{A}_{\ell} \mathrm{f}_{\ell}(r) \\
& =\mathrm{C}_{\ell}^{i} \mathrm{H}_{\ell}^{(1)}\left(\frac{Q x}{\rho}\right)+\mathrm{C}_{\ell}^{r} \mathrm{H}_{\ell}^{(2)}\left(\frac{Q x}{\rho}\right)
\end{aligned}
$$

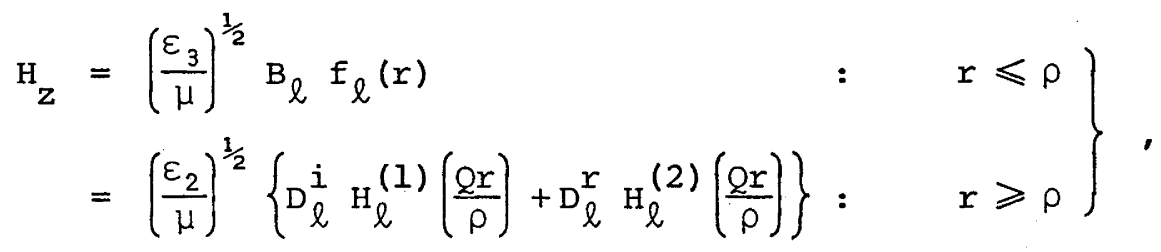

where $\beta$ is the longitudinal propagation constant of the wave parallel to the axis, and

$$
Q^{2}=\rho^{2}\left(k_{2}^{2}-\beta^{2}\right) \geqslant 0
$$

$\varepsilon_{3}$ and $\varepsilon_{2}$ in equation (2) are the dielectric permittivities of the core and cladding either side of the core-cladding interface respectively, as in fig. 7. 


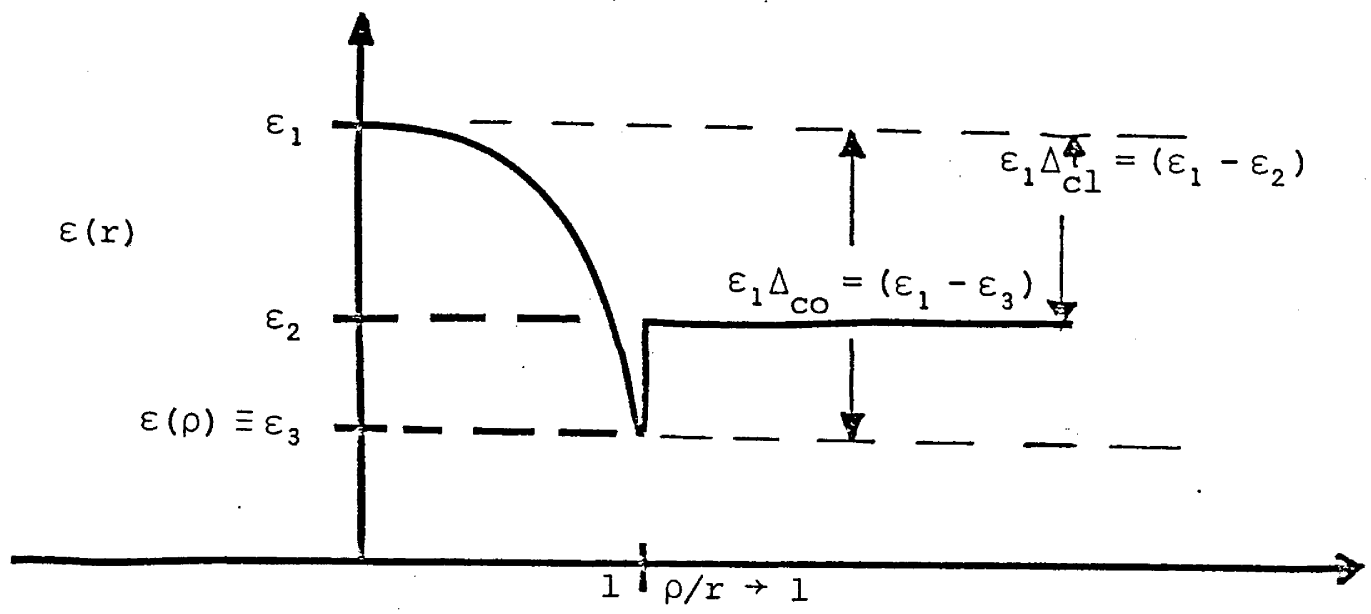

Fig. 7: The dielectric profile of the circular cylindrical dielectric optical fibre. 
Using equations (2A.1)-(2A.4) for the tangential components of the electromagnetic wave, we find

$$
\left.\begin{array}{l}
E_{\phi}=\frac{1}{k_{T}^{2}}\left\{-\frac{\beta \ell}{r} E_{z}+i \omega \mu H_{z}^{\prime}\right\} \\
H_{\phi}=-\frac{1}{k_{T}^{2}}\left\{i \omega \varepsilon E_{z}^{\prime}+\frac{\beta l}{r} H_{z}\right\}
\end{array}\right\},
$$

where

$$
\text { , } \equiv \frac{\partial}{\partial r} \text {. }
$$

We will use the general solutions for the optical waveguide of arbitrary dielectric profile as in chapter 2.

For the incident convergent cylindrical electromagnetic wave with amplitudes $C_{\ell}^{i}, D_{\ell}^{i}$ of the longitudinal electric and magnetic fields, respectively, to be perfectly reflected, we must have the amplitudes of the reflected divergent cylindrical wave satisfying

$$
\left.\begin{array}{l}
\mathrm{C}_{\ell}^{r}=\mathrm{RC}_{l}^{i} \\
D_{l}^{r}=\mathrm{RD}_{\ell}^{i}
\end{array}\right\}
$$

where $R$ is then the reflection coefficient.

Matching the fields defined by equations (1), (2) and (4) across the core-cladding interface at $r=\rho$, we find upon substituting for $\mathrm{A}_{\ell}$ and $\mathrm{B}_{\ell}$,

and

$$
\mathrm{KH}_{\ell}^{(2)} \mathrm{C}_{\ell}^{r}+i \eta_{1}(2) \mathrm{D}_{\ell}^{r}=\mathrm{M}_{1}\left(\mathrm{C}_{\ell}^{i}, \mathrm{D}_{\ell}^{i}\right)
$$

$$
\mathrm{in}_{2}(2) \mathrm{C}_{\ell}^{r}-\mathrm{KH}_{\ell}^{(2)} \mathrm{D}_{\ell}^{r}=\mathrm{M}_{2}\left(\mathrm{C}_{\ell}^{\mathrm{i}}, \mathrm{D}_{\ell}^{i}\right) \text {, }
$$

where

where

$$
\begin{aligned}
& M_{1}\left(C_{\ell}^{i}, D_{l}^{i}\right)=-\left(K H_{\ell}^{(1)} C_{l}^{i}+i n_{1}(1) D_{\ell}^{i}\right) \\
& M_{2}\left(C_{\ell}^{i}, D_{l}^{i}\right)=-i \eta_{2}(1) C_{l}^{i}+K H_{\ell}^{(1)} D_{\ell}^{i},
\end{aligned}
$$

and

$$
K=\frac{\beta l}{k_{2} \rho}\left(\frac{V^{2}(\rho)}{U^{2}(\rho) Q^{2}}\right)
$$


with

$$
\eta_{j}(k)=\left(\frac{a_{j} H_{\ell}^{(k)}}{U^{2}(\rho)} \frac{f_{\ell}^{\prime}}{f_{\ell}}-\frac{1}{\ell^{2}} H_{\ell}^{(k)^{\prime}}\right) \text { for } k=1,2 ; j=1,2
$$

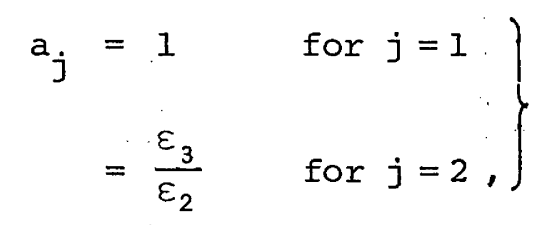

where we have assumed $\mu$ is uniform throughout the core and cladding

and

$$
\left.\begin{array}{l}
U^{2}(\rho)=k_{2} \rho\left(\frac{\varepsilon_{3}}{\varepsilon_{2}}-1\right)^{\frac{1}{2}} \\
U^{2}(\rho)=V^{2}(\rho)+Q^{2}
\end{array}\right\}
$$

The arguments of the Hankel function is $Q$, defined in equation (3).

Equations (6) and (7) represent two simultaneous equations for $\mathrm{C}_{\ell}^{r}$ and $\mathrm{D}_{\ell}^{r}$ in terms of $\mathrm{C}_{\ell}^{i}$ and $\mathrm{D}_{l}^{i}$. Now applying equations (5), we have four simultaneous equations in four unknowns, thus we can find unique solutions for the arbitrary coefficients. Solving these four equations, equations $(4)-(7)$, we find

$$
D_{l}^{i}=-\frac{j\left(\psi_{1}-R \Lambda\right)}{k \chi_{1}} C_{l}^{i}
$$

where we use $j=\sqrt{-1}$ to avoid confusion with the superscripts

and

$$
\begin{aligned}
\psi_{1} & =\left\{n_{1}(2) n_{2}(1)-\kappa^{2} \mathrm{H}_{\ell}^{(1)} \mathrm{H}_{\ell}^{(2)}\right) \\
\Lambda & =\left\{\left(\kappa \mathrm{H}_{\ell}^{(2)}\right)^{2}-n_{1}(2) n_{2}(2)\right\}
\end{aligned}
$$

$$
x_{j}=\left(n_{j}(1) H_{\ell}^{(2)}-n_{j}(2) H_{\ell}^{(1)}\right) \quad \text { for } j=1,2 \text {, }
$$

where the reflection coefficient $R$ in equation (14) is the solution of

and

$$
\left(\psi_{1}-R \Lambda\right)\left(\psi_{2}-R \Lambda\right)=k^{2} \chi_{1} X_{2}
$$

$$
\psi_{2}=\eta_{1}(1) \eta_{2}(2)-k^{2} \mathrm{H}_{\ell}^{(1)} \mathrm{H}_{\ell}^{(2)}
$$

and $X_{2}$ defined in equation (17). 
Equation (18) is a quadratic equation in $R$, thus there are two solutions for every mode number $\ell$.

For weakly guiding fibres $\varepsilon_{2} / \varepsilon_{3} \sim 1$ so that from equation (11)

$$
\eta_{1}(k)=n_{2}(k)
$$

and thus

$$
X_{1}=X_{2} \equiv X=\eta(1) H_{\ell}^{(2)}-\eta(2) H_{\ell}^{(1)}
$$

and

$$
\begin{aligned}
\psi_{1}=\psi_{2} & =n(1) \eta(2)-k^{2} \mathrm{H}_{\ell}^{(1)} \mathrm{H}_{\ell}^{(2)} \\
& =\left(\eta(1) \pm \kappa \mathrm{H}_{\ell}^{(1)}\right)\left(\eta(2) \mp \mathrm{H}_{\ell}^{(2)}\right) \mp \kappa x
\end{aligned}
$$

and equation (18) has solutions

$$
\psi-\mathrm{R} \Lambda=\mp \kappa X
$$

i.e.

$$
R=\frac{(\psi \mp K X)}{\Lambda}
$$

where from equation (16)

$$
\Lambda=\left\{K \mathrm{H}_{\ell}^{(2)}+\eta(2)\right)\left[K \mathrm{H}_{\ell}^{(2)}-\eta(2)\right)
$$

so that using equations (21) $-(23)$ in equation (25)

$$
R=-\left(\frac{\eta(1) \mp \kappa \mathrm{H}_{\ell}^{(1)}}{\eta(2) \mp \kappa \mathrm{H}_{\ell}^{(2)}}\right)
$$

and from equation (24), equation (14) for the relationship between the amplitudes of the longitudinal electric and magnetic fields

where

$$
D_{\ell}^{i}= \pm j C_{\ell}^{i},
$$

$$
j=(-1)^{\frac{1}{2}} \text {. }
$$

Equations (27) and (28) are the required results where the upper and lower signs in both correspond to the two different cylindrical waves that come under reflection and preserve the structure of the incident 
field.

Note that in no way have we needed to assume only step-index waveguides or the paraxial approximation of $\beta / k_{2}$. 


\section{APPENDIX D \\ THE CORRECTED POWER ATTENUATION COEFFICIENT \\ OF THE HE 11 MODE ON A STEP-INDEX FIBRE \\ DUE TO SINUSOIDAL RADIUS FLUCTUATIONS}

From equation. (3.3.38) the Volume Current calculations for the $\mathrm{HE}_{11}$ modal power attenuation coefficient is

where

$$
\begin{aligned}
\alpha= & \frac{\pi a^{2} w_{0}^{2} v^{2}}{8 k_{2} \rho^{2} J_{1}^{2}\left(U_{0}\right)}\left\{J_{0}^{2}\left(U_{0}\right) J_{0}^{2}\left(Q_{r}\right)\right. \\
& \left.+\left(\frac{\beta_{r}}{k_{2}} J_{0}\left(U_{0}\right) J_{0}\left(Q_{r}\right)+\frac{U_{0} Q_{r}}{k_{2} \beta_{0} \rho^{2}} J_{1}\left(U_{0}\right) \cdot J_{1}\left(Q_{r}\right)\right)\right\}^{2},
\end{aligned}
$$

$$
\varepsilon_{r}=k_{2} \rho \sin \theta_{0}
$$

and

$$
u_{r}=\left(v^{2}+Q_{r}^{2}\right)^{\frac{1}{2}}
$$

Now, from this form of the equation, we can identify the amplitudes of the converging and diverging wave components, and from equation (2.16) we find

$$
\mathrm{J}_{\ell}\left(Q_{r}\right) \rightarrow \frac{1}{2}\left(\mathrm{H}_{\ell}^{(2)}\left(\mathrm{q}_{r}\right)+\mathrm{R}(\rho) \mathrm{H}_{\ell}^{(2)}\left(Q_{r}\right)\right)
$$

where now the radiation emanates from the core-cladding interface.

Thus, using equations (2.5) and (2.10) for the reflection coefficient, taking the upper sign for the $\mathrm{HE}_{11}$ radiation, with equation (4) in equation (1), we have, using the general recurrence and Wronksian relations for cylinder function [17]

$$
\alpha=\frac{a^{2} w_{0}^{2} v^{2}}{2 \pi k_{2} \rho^{2} J_{1}^{2}\left(U_{0}\right)} \frac{\Psi\left(U_{r}, Q_{r}\right)}{\left(J_{1}\left(U_{r}\right) U_{r} Q_{r}\right)^{2}\left|\eta(2)-k_{l}^{(2)}\left(Q_{r}\right)\right|^{2}},
$$


$4 . \mathrm{D}$

where $\alpha$ is now the modal power attenuation for the paraxial directed radiation. 


\section{REFERENCES}

[1] A.W. Snyder and D.J. Mitchell: "Leaky rays cause failure of geometric optics in optical fibres", Electron. Letters 9, 437 (1973).

[2] D. Marcuse: "Higher order loss processes and the loss penalty of multimode operation", B.S.T.J. 51, 1819 (1972).

[3] D. Gloge: "Optical power flow in multimode fibers", B.S.T.J. 51, 1767 (1972).

[4] A.W. Snyder: "Radiation losses due to variations of radius of dielectric or optical fibers", I.E.E.E. Trans. MTT-18, 608 (1970).

[5]. D. Marcuse: Theory of Dielectric Optical Waveguides (Academic Press, New York, 1974).

[6] E.G. Rawson: "Analysis of scattering from fiber waveguides with irregular core surfaces", Appl. Optics 13, 2370 (1974).

[7] Ref. 5, pp.155-6; and also "Radiation loss of the $\mathrm{HE}{ }_{11}$ mode of a fiber with sinusoidally perturbed core boundary", Appl. Optics 14, 3021 (1975).

[8] J.A. Stratton: Electromagnetic Theory (McGraw-Hill, New York, 1941).

[9] See ref. 8, p.573 onwards for an excellent summary of this research.

[10] Ref. 8, p.577, equation (20).

[11] D. Marcuse: Light Transmission Optics (Van Nostrand, Reinhold, New York, 1972).

[12] D. Marcuse: private communication.

[13] M. Born and E. Wolf: Principles of Optics (Pergamon, London, 1959).

[14] P. Morse and H. Feshbach: Methods of Theoretical Physics (McGrawHill, New York, 1953), Chapt. 7. 
[15] J.R. Wait: "Scattering of a plane wave from a circular dielectric cylinder at oblique incidence", Can. J. Phys. 33, 189 (1955).

[16] A.W. Snyder: "Continuous mode spectrum of circular dielectric rod", I.E.E.E. Trans. MTT-19, 720 (1971).

[17] M. Abramowitz and I.A. Stegun: Handbook of Mathematical Functions (Dover, New York, 1972), Chapt. 9.

[18] R.A. Sammut: Theory of Unbound Modes on Circular Dielectric Waveguides (Ph.D thesis, Aust. Nat. Univ., 1975), Chapt. 2.

[19] Ref. 8, p.369.

[20] Ref. 8, Chapter 6. 


\section{CHAPTER 5 \\ CURVATURE INDUCED RADIATION : THE SURFACE CURRENT METHOD}

\subsection{INTRODUCTION}

The analysis of the previous chapters has been primarily concerned with the effect of structural and material irregularities in the straight dielectric optical waveguide. In Chapters 3 and 4 we have amply demonstrated that the Volume Current Method, together with the correction to account for the effect of the core-cladding interface, provides an excellent description of these radiative losses while clarifying the physical processes. In this chapter and the next, we consider a different type of radiation mechanism, namely curvature induced radiation, which cannot be treated by the previous philosophy.

In this chapter, we present the Surface Current Method, closely related, conceptually, to the Volume Current Method, and apply this technique to the study of the radiation loss of the modes, both "proper" and "improper" [1], of the straight ideal dielectric optical fibre.

In section 2, we derive the formulae of the Surface Current Method, with particular reference to the weakly guiding dielectric optical fibre of circular cross section, and discuss the underlying philosophy and constraints of the technique. In section 3 , we use this method to derive the modal power attenuation coefficients of the tunnelling leaky modes of the weakly guiding dielectric optical fibre $[1,2]$ to demonstrate the facility of the method. Finally, in section 4, we discuss the limitations of the method and provide a qualitative explanation of the inapplicability of the Volume Current Method to such curvature induced radiation. However, before we immerse ourselves in 
the detail of the method, let us first develop an intuitive understanding of the physical mechanisms of curvature induced radiation.

The study of curvature induced radiation is by no means a new field, with Rayleigh [3] examining the effect of the "whispering gallery" rays of acoustics. However, the study of the electromagnetic equivalent has been a more recent phenomena (with the earliest treatment by Richtmeyer [4] in 1939), with the interest in dielectric waveguides providing impetus. This curvature induced electromagnetic radiation is analogous to the quantum mechanical phenomena of tunnelling of matter waves through finite boundaries [5], and indeed the primary cause of both lie in the wave character of the fields [6-10]. When an electromagnetic wave impinges on a curved dielectric interface from the medium of higher refractive index at an angle greater than the critical angle $\alpha_{C}$, as in fig. 1 , a small fraction of the incident energy appears as free radiation in the medium of lower refractive index. Unlike the refracting rays at planar surfaces, this radiation emanates from a surface away from the interface between the two dielectrics. By geometric optics, this energy should not be transmitted at all, since the energy is incident at an angle, $\alpha$, greater than the critical angle, $\alpha_{C}$. The distance of the surface, from which the radiation emanates, away from the dielectric interface is known as the "caustic" or "turning point".

The primary cause for this electromagnetic tunnelling of the incident wave through the interface is that the dielectric interface, even when planar, does not completely confine the energy of the incident wave to the more dense medium, but allows a small fraction of the incident energy to penetrate into the less dense medium, in the form of an evanescent wave which decays away from the interface, in the transverse plane.

We can interpret the principle of total internal reflection as primarily due to this energy in the less dense medium (the cladding) travelling at a speed less than the speed of light in the cladding since $\beta>k_{2}$. Thus, this energy cannot detach itself from the energy in the more dense region (the core) and radiate away. However, when the interface between the two dielectrics is curved, the energy in the 


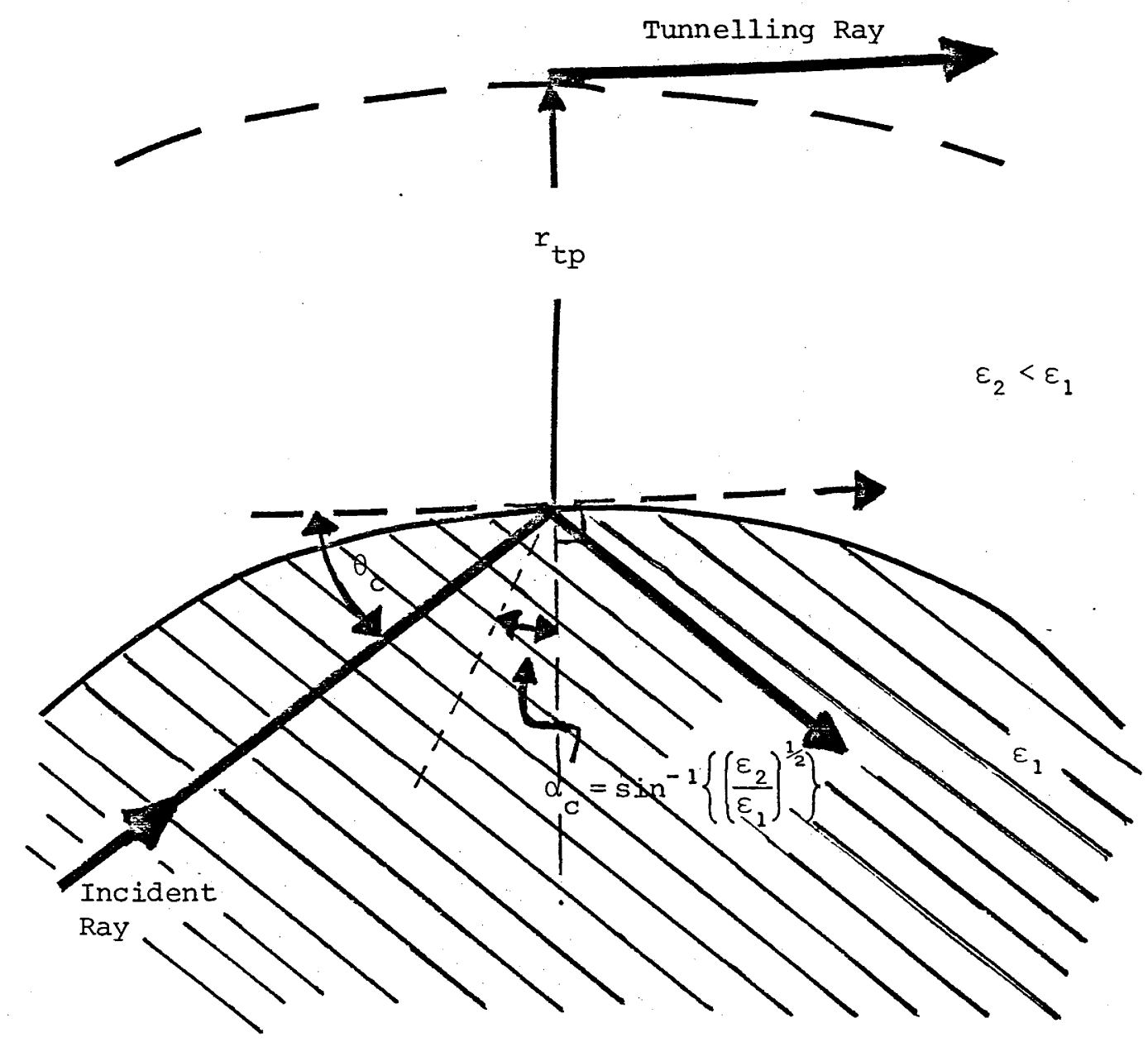

Fig. 1: Ray optical interpretation of the curvature induced radiation loss. The tunnelling leaky ray emerges at a distance, $r_{t p}$ ' from the core-cladding interface. 
cladding, due to the wave incident from the core, must travel at a faster speed the further the energy is from the centre of curvature of the interface, in order to remain in step with the incident wave. At some point then, the speed of the energy in the cladding exceeds the velocity of light in the cladding, and the energy beyond this point can detach itself from the incident energy and radiate away into the cladding. This position is the "turning point" mentioned previously.

Now that we have an understanding of the fundamental principle behind the curvature induced losses, let us proceed with the detailed discussion of the Surface Current Method that we shall eventually apply to these loss processes.

\subsection{THE SURFACE CURRENT METHOD}

In this section we derive the formulae of the Surface Current Method in general and then specialise this result for the practically important case of the weakly guiding dielectric optical fibre of circular cross section. This technique is founded upon the Equivalence Principle, formulated by Schelkunoff [11], or the Stratton-Chu Integral [12] for the fields in a volume $V^{\prime}$, due to the sources in the volume together with the effect of sources outside the volume which can be represented by equivalent surface currents on the surface $S$ ' enclosing the volume. The replacement of the sources outside the volume by equivalent currents over the surface is the philosophy of Huygen's Principle [13], in which the fields over a surface (generated by sources away from the surface) can be considered as secondary sources for the fields beyond the surface, on the opposite side of $S$ ' to the real sources. These pseudo sources generate secondary waves that add to produce distant wavefronts. In the electromagnetic equivalent of Huygen's Principle, these pseudo sources can be considered as electric and magnetic surface current densities distributed over the surface $S^{\prime}$.

From the Stratton-Chu Integral, the electric and magnetic fields in a volume $\mathrm{V}$ at a position $\underset{\sim}{\mathrm{R}}$ can be written as [12] ${ }^{\dagger}$

t It should be noted that due to use of $\exp (i \omega t)$ variation in this 


$$
\begin{aligned}
& \underset{\sim}{E}(\stackrel{R}{\sim})=\frac{1}{4 \pi} \int_{V^{\prime}}\left(-i \omega \mu \underset{\tau^{J}}{J_{e}} \phi-\underset{m_{m}}{J^{\prime}} \times \nabla \phi+\frac{1}{\varepsilon_{2}} \rho_{e} \nabla \phi\right) d V^{\prime} \\
& -\frac{1}{4 \pi} \int_{S^{\prime}}(-i \omega \mu(\underset{\sim}{\hat{\sim}} \times \underset{\sim}{H}) \phi+(\underset{\sim}{\hat{\sim}} \times \underset{\sim}{E}) \times \nabla \phi+(\underset{\sim}{\hat{\sim}} \cdot \underset{\sim}{E}) \nabla \phi) d A^{\prime}
\end{aligned}
$$

and

$$
\begin{aligned}
\underset{\sim}{H(R)}= & \frac{1}{4 \pi} \int_{V^{\prime}}\left(-i \omega \varepsilon_{2} \underset{\sim m}{J} \phi+\underset{\sim e}{J} \times \nabla \phi+\frac{1}{\mu} \rho_{m} \nabla \phi\right) d V^{\prime} \\
& +\frac{1}{4 \pi} \int_{S^{\prime}}\left(-i \omega_{2}(\underset{\sim}{(\hat{n}} \times \underset{\sim}{E}) \phi-(\underbrace{\hat{n}}_{\sim} \times \underset{\sim}{H}) \times \nabla \phi-(\underset{\sim}{\hat{n}} \cdot \underset{\sim}{H}) \nabla \phi\right) d A^{\prime},
\end{aligned}
$$

where $\varepsilon_{2}$ and $\mu$ are the dielectric permittivity and magnetic permeability of the homogeneous isotropic dielectric of volume $V^{\prime}$ enclosed by the surface $S^{\prime} . \phi$ is the Green's function for the homogeneous region and $\hat{\sim}$ is the outward normal to the surface. ${\underset{\sim}{e}}_{e}, \rho_{e}$ and ${\underset{\sim}{m}}_{m}, \rho_{m}$ are the electric and magnetic volume current and charge densities respectively.

If all the electric and magnetic sources are enclosed within a sphere of finite radius, then the field is regular at infinity and the contribution of the surface at infinity can be neglected in the surface integrals of equations ( 1 ).

From an inspection of equation (1), we can see that the terms

and

$$
\underset{\sim}{\mathrm{K}}=-\underset{\sim}{\hat{\sim}} \times \underset{\sim}{\mathrm{H}}
$$

$$
\underset{\sim m}{\mathrm{~K}}=\underset{\sim}{\hat{\sim}} \times \underset{\sim}{\mathrm{E}}
$$

behave as if they were the two-dimensional analogues of the electric and magnetic volume current densities, $J_{e}$ and $J_{m}$ respectively. Similarly, the terms $(-\hat{\sim} \cdot \underset{\sim}{\mathbb{N}})$ and $(-\hat{\sim} \cdot \underset{\sim}{\sim})$ appear as the two-dimensional counterparts of the volume electric and magnetic charge densities. Thus $\underset{\sim}{\mathrm{K}}$ and $\underset{\sim}{\mathrm{K}}$ are referred to as the pseudo surface current densities of electric and magnetic character, respectively. Thus, for a region in which there are no real sources, the field in the volume is generated by the sources beyond the boundary to the volume and these sources can be represented

analysis, in comparison to exp (-iwt) variation in ref. 12 , there are minor discrepancies in sign between the two formulae. 


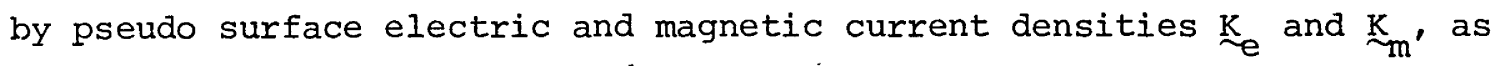
defined in equation (2).

In general, the complete description of the field inside the volume $V^{\prime}$ is of little interest. For the special situations for which only the knowledge of the radiation fields that exist far from the surface $S^{\prime}$ is required, the formulation is greatly simplified and the radiation fields (denoted by the superscript $R$ ) become (Appendix $A$ )

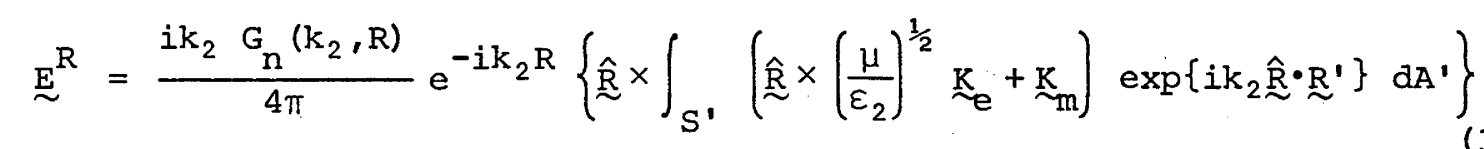

and

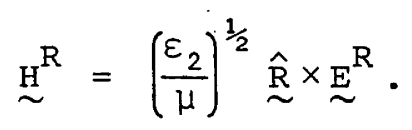

$G_{n}\left(k_{2}, R\right)$ is obtained from the asymptotic form of the Green's function for the homogeneous isotropic medium of $n$ dimensions [14]

$$
\begin{aligned}
G\left(k_{2}, R\right) & =\left(\frac{2 \pi}{k_{2} R}\right)^{\frac{1}{2}} & \text { for } n=2 \\
& =\frac{1}{R} \quad & \text { for } n=3 .
\end{aligned}
$$

$\underset{\mathrm{R}}{\mathrm{R}}$ is the unit vector in the direction of the observation point in the radiation field, from the origin. $\mathbb{R}^{\prime}$ is the position vector of points on the surface $S^{\prime}$ and $k_{2}\left(=\omega\left(\mu \varepsilon_{2}\right)^{\frac{1}{2}}\right)$ is the wavenumber of light in the cladding.

which becomes

From equations (3) and (4) the time average radial Poynting vector, $S^{R}$, for the power radiating away from the surface $S^{\prime}$ is [12]

$$
S^{R}=\frac{1}{2} \underset{\sim}{E} \times \underset{\sim}{H^{*}} \cdot \underset{\sim}{\hat{R}}=\frac{1}{2}\left(\frac{\varepsilon_{2}}{\mu}\right)^{\frac{1}{2}}\left|\underset{\sim}{E^{R}}\right|^{2}
$$

$$
S^{R}=\left(\frac{\varepsilon_{2}}{\mu}\right)^{\frac{1}{2}} \frac{k_{2}^{2}}{32 \pi^{2}}\left|G_{n}\left(k_{2}, R\right)\right|^{2}\left|\left\{\underset{\sim}{\hat{R}} \times \int_{S^{\prime}}\left(\underset{\sim}{\hat{R}} \times\left(\frac{\mu}{\varepsilon_{2}}\right)^{\frac{1}{2}} \underset{\sim}{\mathbb{N}_{e}}+\underset{m_{m}}{K_{m}}\right) \exp \left\{i k_{2} \underset{\sim}{\hat{R}} \cdot \underset{\sim}{R^{\prime}}\right\} d A^{\prime}\right\}\right|^{2}
$$

and the total power radiated $\mathrm{P}^{\mathrm{R}}$ can be determined by integrating $\mathrm{S}^{\mathrm{R}}$ over the surface of a sphere of radius $R \rightarrow \infty$ enclosing the surface $S^{\prime}$ [12], 


$$
\left.\begin{array}{rlr}
P^{R} & =R^{2} \iint S^{R} \sin \theta d \theta d \phi: & \text { for } 3 \text { dimensions } \\
& =R \cdot \int S^{R} d \theta \quad: \quad \text { for } 2 \text { dimensions }
\end{array}\right\} \text {. }
$$

From equations (8) and (9), it is obvious that the knowledge of the fields over the surface $S^{\prime}$ is sufficient to determine the total radiated power. Although the form of equation (9) is intimidating for all but the most elementary of surfaces and incident fields, we shall see that for the weakly guiding dielectric optical fibre of circular cross section, the calculation can be considerably simplified. Equations (8) and (9) form the basic formulae of the surface Current Method by which the effect of the core of the optical waveguide and all sources within the core are replaced by pseudo-surface currents distributed over the core-cladding interface, ${ }^{\dagger}$ expressed in terms of the modal fields on the fibre which are excited by the sources in the core.

By a judicious choice of the electromagnetic modes of the weakly guiding optical fibre of circular cross section, the analysis, using the integrals defined in equation (8), can be considerably reduced. In fact, these calculations via the Surface current Method were the determining factors in the choice of the azimuthal travelling wave modal form, developed in Chapter 2. These azimuthal travelling waves correspond to rays travelling in a skew path in the $\pm \phi$ directions around the axis, as the rays propagate along the fibre, depending on whether it is $\pm l$ modes considered.

The enormous reduction in the volume of calculations necessary in the Surface Current Method when the azimuthal travelling wave modes are used arises from the simple relation between the electric and magnetic fields of the mode, i.e. equation (2.2.46), viz.

$$
\underset{\mathrm{H}}{\sim}= \pm i\left(\frac{|\ell|}{\ell}\right)\left(\frac{\varepsilon_{2}}{\mu}\right)^{\frac{1}{2}} \underset{\sim}{\mathrm{E}},
$$

where the azimuthal variation is $\exp \{-i \ell \phi\}$. The + and - signs refer to

\footnotetext{
To be exact, these currents must be considered over a surface just outside the core-cladding interface, in the cladding so that the criteria for the Stratton-Chu Integral are satisfied.
} 
the $\mathrm{HE}_{\ell}$ and $\mathrm{EH}_{\ell}$ modes respectively.

Using equation (10) in equation (8) for the time averaged Poynting vector for the radiation from the azimuthal travelling wave, we find that we need only calculate one vector integral, $\underset{\sim}{\sim}$ in comparison to the two previously necessary, and (Appendix B)

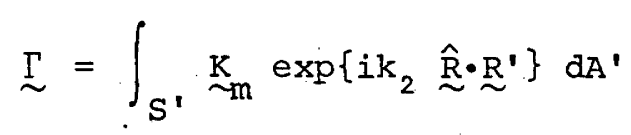

and the time averaged Poynting vector $s^{R}$ is

$$
S^{R}=\left(\frac{\varepsilon_{2}}{\mu}\right)^{\frac{1}{2}} \frac{k_{2}^{2}}{16(\pi R)^{2}}\left|\underset{\sim}{\Gamma} \cdot\left(\underset{\sim}{\hat{x}} \pm i \frac{|l|}{l}(\underset{\sim}{\hat{y}} \cos \theta-\hat{z} \sin \theta)\right)\right|^{2},
$$

where we have used the azimuthal symmetry of the modes and the optical fibre itself, so that we need only calculate the radiation at one position in the azimuthal plane, viz. $\phi=\pi / 2$, see fig. 2. The + and signs in equation (12) refer to the $\mathrm{HE}_{\ell}$ and $\mathrm{EH}_{\ell}$ modes respectively.

From a comparison of equation (12) and equation (8) one can readily see why the azimuthal travelling wave mode is chosen in preference to the standing wave mode form, i.e. $\cos l \phi$, sin $l \phi$ modes. However, the conclusions drawn from equation (12) cannot be extrapolated to the more physically realistic mode set, i.e. the azimuthal standing wave modes, without justification. The recent experience with the non-uniformity of the attenuation coefficients due to bends in optical fibres for the different polarisations of the IP modes [15,16] should be sufficient motivation for the need for justification of this extrapolation.

In Appendix B, the time averaged Poynting vector of an incident azimuthal standing wave mode is shown to be comprised of two terms, namely the individual power radiated from each of the two azimuthal travelling wave modes (equation B.12). Thus the azimuthal travelling wave components of the standing wave mode radiate independently. As equation (B.12) illustrates, the radiative loss of the $\sin \ell \phi$ and $\cos \ell \phi$ modes for either the $\mathrm{HE}_{\ell}$ or $\mathrm{EH}_{\ell}$ modes are identical. In circumstances in which the symmetric optical waveguide and its distortion are such that there is no discrimination between the $\pm l$ 
travelling wave modes, these two modes should radiate equally and hence the power radiated from a standing wave mode should be twice the radiation from each azimuthal travelling wave mode, and thus the attenuation coefficients should be identical.

We have now derived the formulae for the Surface Current Method, equations (8), (9) and (12). Theoretically, the technique is exact with the proviso that the fields of the surface $S^{\prime}$ are known exactly. In the derivation of the formulae the only restriction is that the volume in which the fields are required must be a homogeneous isotropic region [12]. Thus for multiply clad dielectric waveguides one must be careful to apply the integrals over the outermost surface. Similarly, the analysis is such as to be able to study graded-index fibres with an homogeneous cladding, and equation (8) is not confined to round optical waveguides although equation (12) is specialised to waveguides of circular cross section.

Before we begin to apply the Surface Current Integrals to radiative loss processes, we shall recall one crucial result. From equation ( $(\mathrm{a}),(\mathrm{lb})$, the electromagnetic fields in the volume $V^{\prime}$ are linearly related to fields at the surface $S^{\prime}$ so that the accuracy of these surface fields, which are used in the pseudo surface currents, is the fundamental limitation to the accuracy of the fields in the volume $\mathrm{V}^{\prime}$.

Let us now consider one of the few problems in which the field at the waveguide surface is known to a high degree of accuracy, i.e. radiation from the modes of the straight ideal dielectric optical fibre.

\subsection{RADIATION FROM THE IDEAL STRAIGHT OPTICAL FIBRE}

In this section, we use the formulae of the surface current Method, equations (9) and (12) to determine the power radiated from a mode of the straight optical fibre in a length, $2 \mathrm{~L}$, of fibre, as in fig. 2, where

$$
\mathrm{k}_{2} \mathrm{~L} \gg \mathrm{l}
$$


5.3

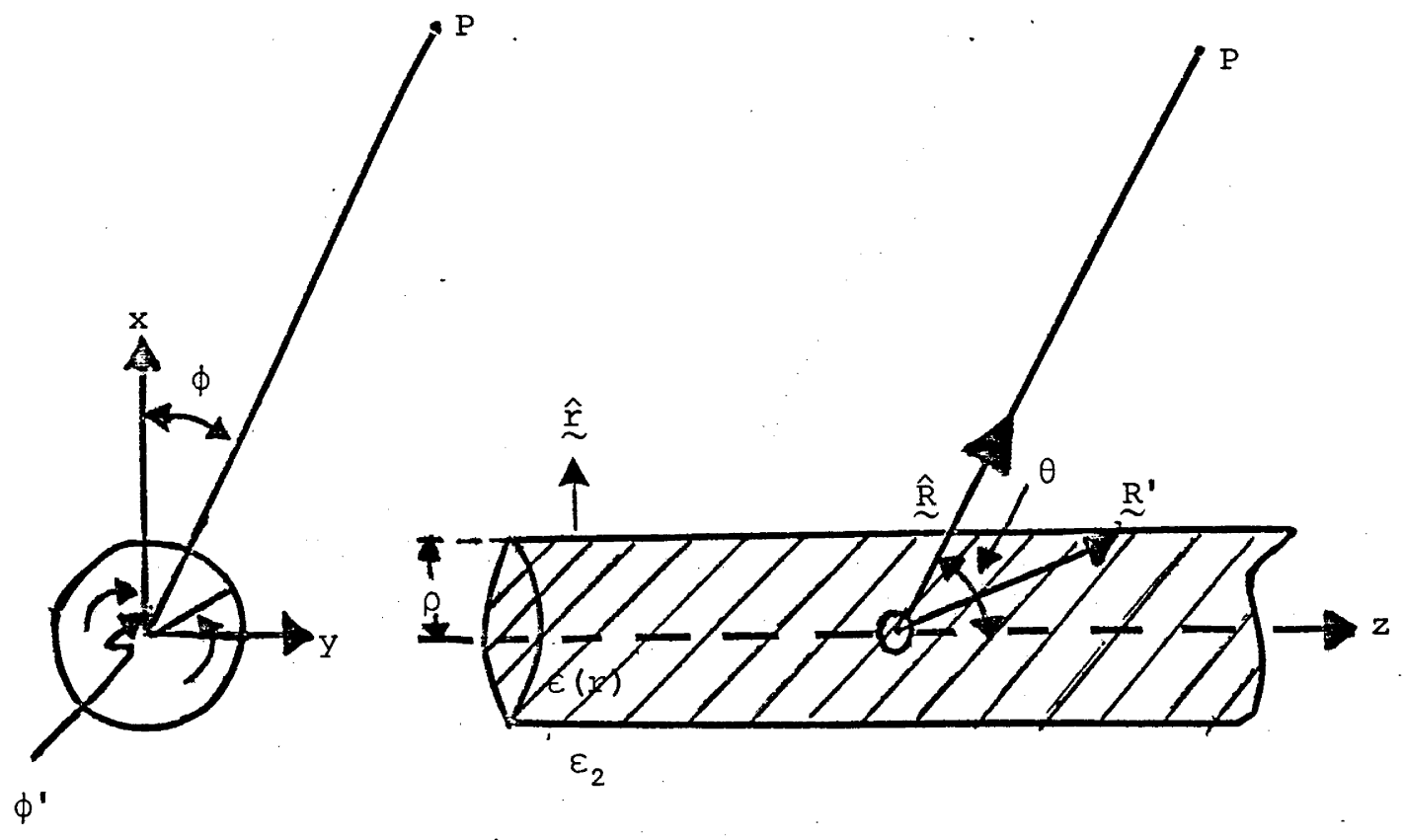

Fig. 2: Co-ordinate system for the circular cylindrical dielectric optical fibre. 
Although we consider only a finite segment of fibre we will ignore the contributions due to the surfaces at the ends of the fibre, i.e. the end faces of the finite segment. This is ignored since the power radiated from the end faces is both an artificial loss process and as it is not strongly length dependent, * will not contribute to the power attenuation coefficient of the fibre, $\alpha$, where

$$
\alpha=\frac{P^{R}}{2 L \cdot P_{0}},
$$

where $P^{R}$, the radiated power, is assumed to be small in comparison to the incident guided power, $\mathrm{P}_{0}$, i.e.

$$
\mathrm{P}^{\mathrm{R}} \ll \mathrm{P}_{0}
$$

In the derivation, we shall be considering both the "proper" bound modes of the structure as well as the analytic continuation of these modes, together with their modal properties such as the eigenvalue equation, etc., below cutoff, where they become the tunnelling leaky modes of the optical fibre [2-3]. Although in a strict physical and mathematical sense, such an analytic continuation poses problems as to the interpretation of some of the physical parameters, such as the power normalisation coefficient, $A_{\ell \mp 1}$ from equation $(2.2 .53)$, we show that the results provide excellent agreement with previous analysis.

For the weakly tunnelling leaky modes of the fibre, the interpretations of the analytically continued parameters, mentioned above, have been shown by Snyder and Mitchell to have sound physical meanings. As discussed previously, we will choose the core-cladding interface to be the region over which the surface integrals will be evaluated, as in fig. 2 .

\footnotetext{
$+k_{2} L$ is large enough so that the radiation pattern is sharp but not long enough so that the incident unattenuated modal field is no longer a valid approximation to the field along the length, $2 \mathrm{~L}$, of fibre. [This note refers to bottom of p.167.]

* The only length dependence of the end face contributions is due to the interference between the radiation from the ends. The absolute magnitude of the radiation is reasonably unaffected by the length of the segment.
} 
In the travelling azimuthal wave mode formulation the vector integral, $\underset{\sim}{\sim}$, defined in equation (2.11), becomes (Appendix $C$ ) for the weakly guiding fibre modes defined by equation $(2.2 .43)-(2.2 .48)$

$$
\begin{aligned}
& \underset{\sim}{\sim}=\mathrm{A}_{\ell \mp 1} \rho \int_{0}^{2 \pi} \mathrm{d} \phi^{\prime} \int_{-L}^{L} \mathrm{~d} z^{\prime}\left\{\left(\exp \left\{i\left(k_{2} \rho \sin \theta \sin \phi^{\prime}+\left(k_{2} \cos \theta-\beta\right) z^{\prime}-\ell \phi^{\prime}\right)\right\}\right.\right.
\end{aligned}
$$

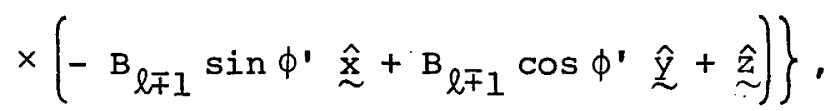

where $A_{\ell \mp 1}$ is the power normalisation coefficient, which after using the integral representation of Bessel functions and the recurrence relations [17],

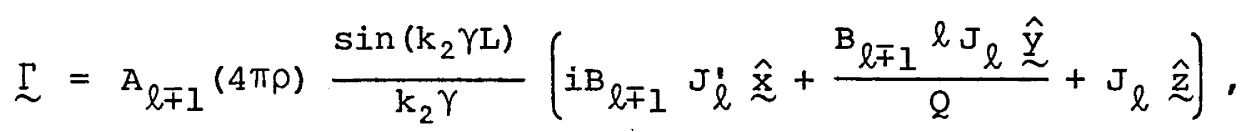

where the argument of the Bessel functions is $Q$, where

$$
Q=k_{2} \rho \sin \theta
$$

and

$$
B_{\ell \mp 1}=\frac{i W}{k_{2} \rho} \frac{{ }_{\ell}^{(1)}(i W)}{H_{\ell \mp 1}^{(1)}(i W)}
$$

where the subscripts $(\ell-1)$ and $(\ell+1)$ refer to the $\mathrm{HE}_{\ell}$ and $\mathrm{EH}_{\ell}$ modes respectively, and

$$
\mathrm{k}_{2} \gamma=\mathrm{k}_{2} \cos \theta-\beta
$$

Using equation (3) in equation (2.14) and integrating $S^{R}$ obtained from equation (2.14) to obtain $\mathrm{P}^{\mathrm{R}}$, the total power radiated, we find (Appendix C)

$$
\mathrm{P}^{\mathrm{R}}=2 \mathrm{~L}\left(\frac{\varepsilon_{2}}{\mu}\right)^{\frac{1}{2}} \cdot \pi^{2} \mathrm{k}_{2} \rho^{2} \mathrm{~A}_{\ell \mp 1}^{2}\left\{\mathrm{~B}_{\ell \mp 1} \mathrm{~J}_{\ell}^{\prime} \pm \mathrm{J}_{\ell}\left(\frac{\mathrm{B}_{\ell \mp 1} \beta \ell}{Q}-\frac{Q}{\mathrm{k}_{2} \rho}\right)\right\}^{2}
$$

if

Otherwise

$$
\cos \theta_{0}=\frac{\beta}{k_{2}} \leqslant 1
$$

$$
\mathrm{P}^{\mathrm{R}}=\mathrm{O}\left(\frac{1}{\mathrm{k}_{2} \mathrm{~L}}\right) \rightarrow 0 \text { as } \mathrm{k}_{2} \mathrm{~L} \rightarrow \infty \text {, }
$$


where from equations (4) and (8)

$$
Q^{2}=\rho^{2}\left(k_{2}^{2}-\beta^{2}\right)=-w^{2} \geqslant 0 \text {. }
$$

From equation (8), for the condition for significant length dependent radiation, we can see that only the modes for which

$$
\mathrm{k}_{2}>\beta
$$

radiate strongly. The bound modes of the fibre satisfy the opposite result to equation (9) and hence do not radiate significantly to contribute to the power attenuation coefficient.

Now, if we seek to find the power radiated from the weakly tunnelling leaky modes which correspond to $\beta / k_{2} \leqslant 1$ [1-2], then using this approximation together with the recurrence relations for Bessel functions and their Wronksian relations [17], $\mathrm{P}^{\mathrm{R}}$ becomes (Appendix C)

$$
P^{R}=\frac{8 L}{k_{2}}\left(\frac{\varepsilon_{2}}{\mu}\right)^{\frac{1}{2}} \quad\left(A_{l \mp 1}\right)^{2}\left(H_{l \mp l}^{(I)}(Q)\right)^{-2},
$$

where we have used

$$
\left.\begin{array}{rl}
Q & =k_{2} \rho \sin \theta_{0} \\
& =\rho\left(k_{2}^{2}-\beta^{2}\right)^{\frac{1}{2}}
\end{array}\right\} .
$$

The modal power attenuation coefficient, $\alpha$, is for those modes which were incident with unit power

$$
\alpha=\frac{P^{R}}{2 L}
$$

and thus

$$
\alpha=\frac{4}{\mathrm{k}_{2}}\left(\frac{\varepsilon_{2}}{\mu}\right)^{\frac{1}{2}}\left(\mathrm{~A}_{\ell \mp 1}\right)^{2}\left(\mathrm{H}_{\ell \mp 1}^{(I)}(Q)\right)^{-2} .
$$

Using the expressions for $A_{\ell \mp 1}$ defined by the analytic continuation of equation (3.3.16) to imaginary $W$ via $W \rightarrow$ iQ for the step-index fibre, equation (12) becomes for the power attenuation coefficient, for the step-index fibre, $\alpha_{\text {s.I. }}$

$$
\alpha_{\text {S.I. }}=\frac{4}{\pi k_{2} \rho^{2}}\left(\frac{U}{V}\right)^{2}\left(H_{\ell \mp 2}^{(I)}(Q) H_{\ell}^{(I)}(Q)\right)^{-1},
$$


where $Q=k_{2} \rho \sin \theta_{0}$, with $\theta_{0}<1$. Equation (13) agrees exactly with the results of Snyder and Mitchell, using a wide range of techniques [18]. The upper and lower signs refer, as usual, to the $\mathrm{HE}_{\ell}$ and $\mathrm{EH}_{\ell}$ modes respectively.

There is one major difference between the calculations of the Surface Current Method and the Volume Current Method. This difference is due to the former technique retaining the longitudinal components to provide an accurate answer, whereas in the Volume Current Method (due to their small size of the longitudinal field), they did not contribute significantly to the scattered energy. At first sight, this appears to be rather surprising until one realises that the surface Current Method is equivalent to calculation of the time averaged Poynting vector in the transverse plane evaluated at the core-cladding interface [18]. Once this is realised, it is obvious that the longitudinal fields, though small, cannot be neglected since those components are necessary to contribute to the real transverse component of the time averaged Poynting vector of the mode.

Although we derived the modal power attenuation coefficient for the modes of the fibre by analytically continuing the modal fields below cutoff, we could have used another procedure that removes the need to re-interpret the power normalisation coefficient $A_{\ell \mp 1}$. By assuming a distribution over the surface of the guide of the form of the proper modes, but with $\beta<k_{2}$, we could have evaluated a temporal attenuation coefficient (i.e. the mode field is everywhere uniform but decaying in time). Using the "equivalence" between the spatial and temporal. attenuation, following snyder and Mitchell [18], where the only analytic continuation is that of the group velocity of a mode below cutoff, we could then obtain the spatial attenuation coefficient without the necessity of re-interpretation of the power normalisation coefficient for modes below their cutoff.

Due to the abundant literature on these tunnelling leaky modes in step-index fibres, we shall not loiter on this topic. The reader is referred to refs. 1 and 18 for complete information on the physics of the tunnelling leaky modes. Having demonstrated the ability of the Surface Current Method for calculations of curvature loss of both step- 
and graded-index waveguides, we shall now discuss in general the applicability of the analysis of the surface Current Method.

\subsection{CONCLUSIONS}

We have presented the philosophy of the Surface Current Method and displayed the ability of the method in the calculation of the leaky mode power attenuation coefficients of the ideal straight guide. The analysis of Appendix $C$ readily demonstrates that the radiative loss calculation does not discriminate between optical fibres with different dielectric profiles of the core. It is only through the power normalisation coefficient, ${ }^{A}{ }_{\ell \mp 1}$, and the fibre and modal parameters, $\rho$ and $Q$ respectively, that a mode behaves differently in different dielectric optical fibres with the same normalised frequency, $v$, where

$$
\mathrm{V}=\mathrm{k}_{\mathrm{l}} \rho \theta_{\mathrm{C}}
$$

where $\theta_{C}$ is the maximum acceptance angle of the core.

In Appendix B, we have also displayed the attractive features of the azimuthal travelling wave modal representation of the incident modal field. The formula for the power radiated from such a mode, equation (2.12), is much simpler than the general result, equation (2.8). To this time, we have not fully explained why the Volume Current Method does not work for curvature induced radiation nor when the surface Current Method is inapplicable. To begin, let us consider the Volume Current Method and its inapplicability to the calculation of curvature induced losses.

Immediately, from the example in section 5.3 of the radiation loss from the tunnelling leaky modes, we can observe that there are no dielectric or structural irregularities to induce any volume dipole moments. Thus, the Volume Current Method would predict that such modes do not radiate. In general, however, if one considers a bent waveguide such as fig. 3 below, we can see that the regions in which the bent waveguide does not conform to the ideal straight waveguide are no longer small, so that no longer can the incident modal fields be used to 


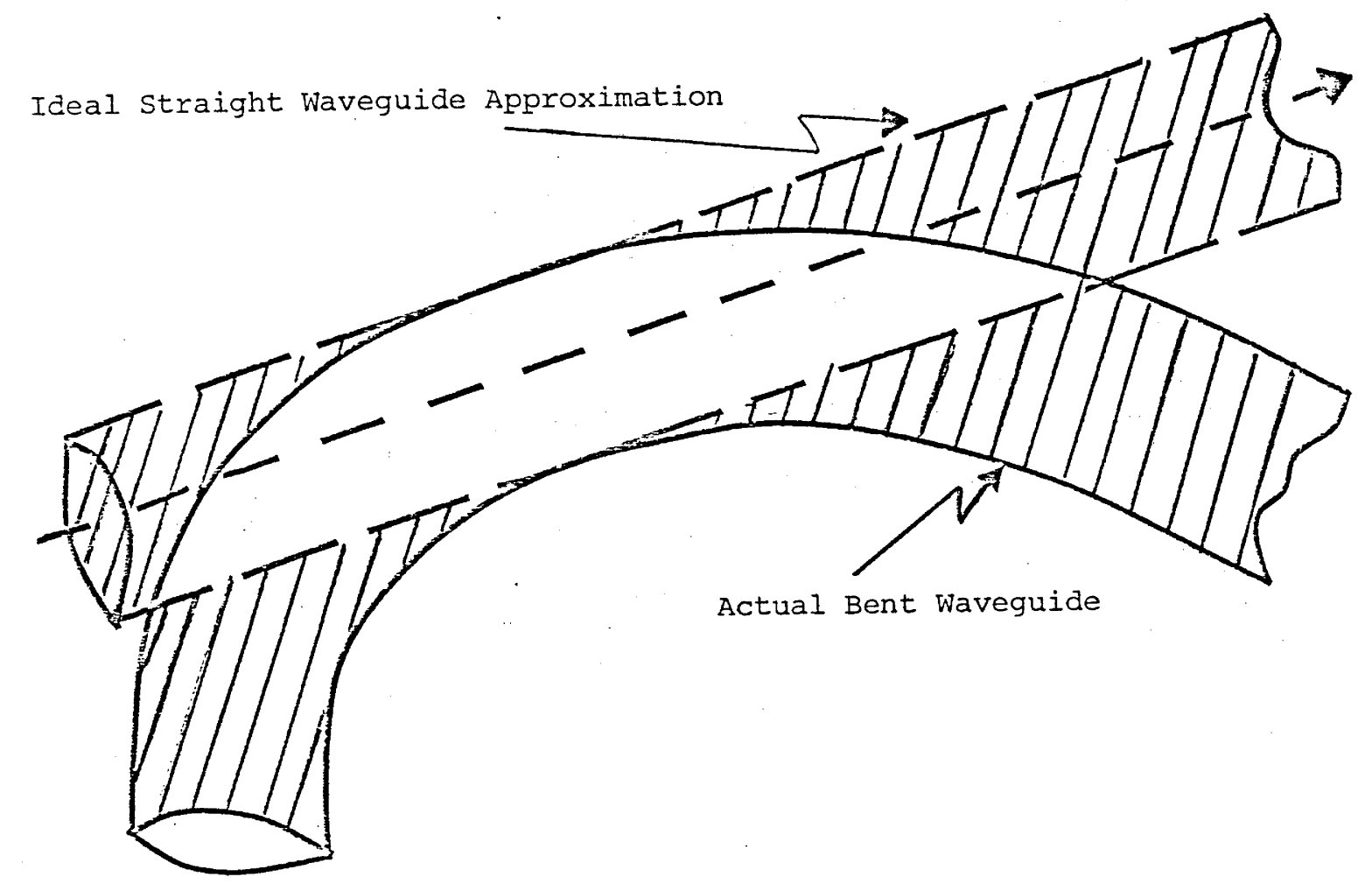

Fig. 3: The shaded regions indicate the perturbation to the ideal waveguide. The perturbation of the bent waveguide is no longer slight with respect to the ideal straight guide. 
approximate the fields at the location of the irregularity. Thus, the Volume Current Method can not be used for such analyses. For slight deviations of the axis of the waveguide about the straight waveguide, as in fig. 4, however, the Volume Current Method can still be used, since everywhere the perturbation to the ideal straight waveguide is small. Rawson [20] has studied such sinusoidal and helical meanders of the waveguide axis and obtains results not too dissimilar to the sinusoidal variations of the radius of a straight fibre discussed in Chapters 3 and 4. Now that we have explained the inapplicability of the Volume Current Method to the curvature induced radiation mechanisms, we shall examine the applicability of the Surface Current Method to a wider range of examples than curvature induced radiation.

As was pointed out in section 5.2, the Surface Current Method as expressed by equation (2.8) is in theory an exact technique, given that the fields on the surface are known exactly. When a mode of the straight optical waveguide is incident on a perturbed segment of the guide, the mode scatters power into the other bound mode of the structure along with power scattered into the radiation fields. Therefore, to determine the radiation field from the perturbed optical waveguide, the coupling into the radiation field must be determined before the Surface Current Method can be applied. However, once the magnitude of the coupling into the radiation field is known, the problem is solved as this directly allows for the determination of the radiative losses.

When such coupling to the radiation fields of the ideal straight structure is negligible, then the incident modal field should provide an adequate description of the modal field in the deformed section [21]. We discuss this further in section 6.5. Such is the case in slow bends in optical waveguides for which the radius of curvature of the optical fibre is large [21]. Now that we have discussed the philosophy and applicability of the Surface Current Method, we shall now apply it to the bending losses of dielectric optical waveguides due to a bend with a constant radius of curvature $R_{0}$, sufficiently large to prevent the breakdown of the above approximation. 


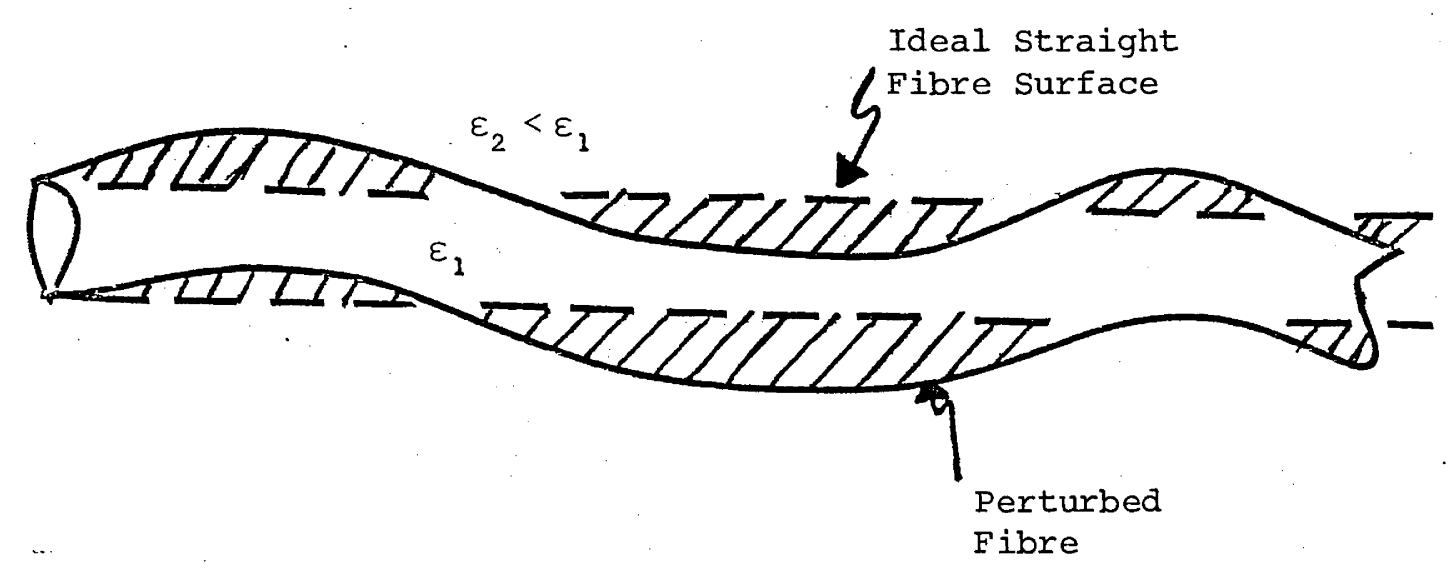

Fig. 4: A dielectric optical waveguide with a slight meander of the axis of the guide. 


\section{APPENDIX A \\ THE RADIATION FIELDS OF THE STRATTON-CHU INTEGRAL}

In this appendix, we use the well known result of the Stratton-Chu Integral for the fields in a volume $V^{\prime}$, due to sources outside the volume, to derive expressions for the radiation field, i.e. the fields far from the surface, $S^{\prime}$, enclosing the volume. From equation (2.1), the electric field at the point with position vector, $\underset{\sim}{\mathrm{R}}$ is

$$
\underset{\sim}{\mathrm{E}} \underset{\sim}{\mathrm{R}})=-\frac{1}{4 \pi} \int_{S^{\prime}}\left\{i \omega \mu \underset{\sim}{\mathrm{K}} \phi+\underset{\sim}{\mathrm{K}} \times \nabla^{\prime} \phi+\underset{\sim}{\hat{\mathrm{n}}} \cdot \underset{\sim}{\mathrm{E}} \nabla^{\prime} \phi\right\} \cdot d A^{\prime},
$$

where the ' indicates the source points that lie on the surface $S$ ', and $\phi$ is the Green's function for the homogeneous, isotropic volume with permittivity $\varepsilon_{2}$, and magnetic permeability $\mu . \underset{\sim}{\underset{\sim}{K}}$ and $\underset{\sim}{\mathrm{K}}$ are the pseudo surface electric and magnetic current densities defined by equations $(2.2)$.

From Maxwell's equations

$$
\nabla \times \underset{\sim}{\mathrm{H}}=i \omega \varepsilon \underset{\sim}{\mathrm{E}}
$$

and using equations (2) in (1), we have

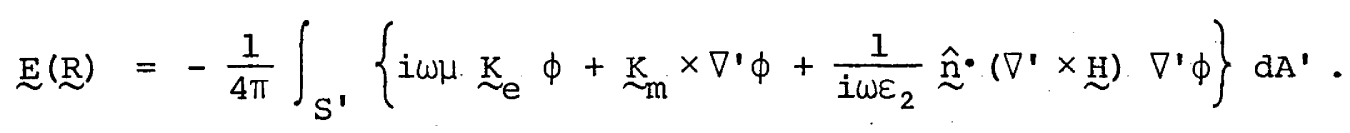

Now, considering equation (3), the last term in the integrand can be re-written as,

$$
\frac{1}{i \omega \varepsilon_{2}} \int_{S^{\prime}} \stackrel{\hat{n}}{\sim} \cdot(\nabla \times \underset{\sim}{H}) \nabla \phi d A^{\prime}=\frac{1}{i \omega \varepsilon} \int_{S^{\prime}}, \hat{\stackrel{\hat{n}}{\sim}} \cdot\left(\underset{\sim}{H} \times \nabla^{\prime} \nabla^{\prime} \phi\right) d A^{\prime},
$$

where we have used Gauss's Theorem [14] to derive 


$$
\int_{S^{\prime}} \stackrel{\hat{n}}{\sim} \nabla \times \underset{\sim}{M} d A^{\prime}=\int_{V^{\prime}} \nabla \cdot(\nabla \times \underset{\sim}{M}) \cdot d V \equiv 0:
$$

Changing the order of the scalar and vector products in equation (4), we have

$$
\frac{1}{i \omega \varepsilon_{2}} \int_{S^{\prime}} \underset{\sim}{\hat{n}} \cdot\left(\nabla^{\prime} \times \underset{\sim}{H}\right) \nabla^{\prime} \phi d A^{\prime}=\frac{1}{i \omega \varepsilon_{2}} \int_{S^{\prime}}(\stackrel{(\hat{\sim}}{\sim} \times \underset{\sim}{H}) \cdot \nabla^{\prime} \nabla^{\prime} \phi d A^{\prime}
$$

which from the definition of $\underset{\sim}{\mathrm{K}}$ in equation (2.2a), equation (5) becomes

$$
\frac{1}{i \omega \varepsilon_{2}} \int_{S^{\prime}} \underset{\sim}{\hat{n}} \cdot\left(\nabla^{\prime} \times \underset{\sim}{H}\right) \nabla^{\prime} \phi d A^{\prime}=-\frac{1}{i \omega \varepsilon_{2}} \int_{S^{\prime}} \underset{\sim}{\stackrel{K}{\sim}} \cdot \nabla^{\prime} \nabla^{\prime} \phi d A^{\prime}
$$

and thus, equation (3), for the electric field, can be written as

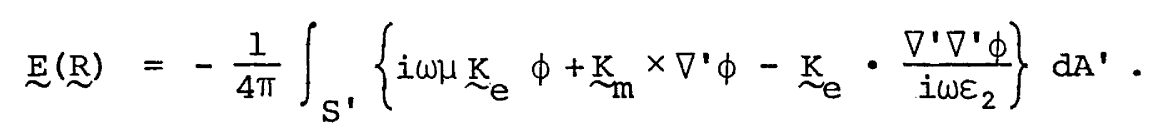

For the radiation field, in which $|\underline{r}|>\left|\underline{r}^{\prime}\right|$, where $\underline{r}^{\prime}$ is the position vector of the points on the surface $S^{\prime}$, we can evaluate $\phi, \nabla^{\prime} \phi$, $\nabla \cdot \nabla \cdot \phi$ asymptotically, so that [14]

where

$$
\phi \sim \phi_{a}=G_{n}\left(k_{2}, R\right) e^{-i k_{2}\left|R-R^{\prime}\right|},
$$

and

$$
\left.\begin{array}{rlrl}
G_{n}\left(k_{2}, R\right) & =\left(\frac{2 \pi}{k_{2} R}\right)^{\frac{1}{2}} & & \text { for } n=2 \\
& =\frac{1}{R} & & \text { for } n=3
\end{array}\right\}
$$

$$
R=\lim _{|r| \rightarrow \infty}\left|\underset{\sim}{R}-\underset{\sim}{R^{\prime}}\right|
$$

where the subscript, $n$, indicates the dimensions of the space considered. The subscript, a, in equation (8), will be used to denote the asymptotic form of the Green's function. From equation (8)

$$
\left.\begin{array}{rl}
\nabla \cdot \phi_{a} & =-\nabla \phi_{a}=i k_{2} \underset{\sim}{\stackrel{R}{R}} \phi_{a} \\
\nabla \cdot \nabla \cdot \phi_{a} & =-k_{2}^{2} \underset{\sim}{\hat{R}} \hat{\sim} \phi_{a}
\end{array}\right\},
$$

where $k_{2}$ is the wavenumber of the light in the medium 


$$
\mathrm{k}_{2}=\omega\left(\mu \varepsilon_{2}\right)^{\frac{1}{2}}
$$

and $\underset{\sim}{\stackrel{R}{R}}$ is the unit vector from the origin to the position in the radiation field. Using a Taylor series expansion for the exponent of $\phi_{a}$ defined in equation (8), we may write

$$
\phi_{a}=G_{n}\left(k_{2}, R\right) e^{-i k_{2} R} \cdot e^{+i k_{2} \underset{\sim}{\stackrel{R}{*}} \cdot R^{\prime}}
$$

and thus from equations (8) - (12), equation (7) becomes

$$
\begin{aligned}
& \underset{\sim}{E^{R}}=e^{-i k_{2} R} \frac{i k_{2} G_{n}\left(k_{2}, R\right)}{4 \pi} \int_{S^{\prime}}\left[\underset{\sim}{\hat{R}} \times\left\{\underset{\sim}{\stackrel{R}{\sim}} \times\left(\frac{\mu}{\varepsilon_{2}}\right)^{\frac{1}{2}} \underset{\sim}{\underset{\sim}{K}}+\underset{m}{\mathrm{~K}}\right\}\right] \\
& \times \exp \left(i k_{2} \underset{\sim}{\stackrel{R}{R} \cdot R^{\prime}}\right) d A^{\prime}
\end{aligned}
$$

and from an identical analysis for $\underset{\sim}{\mathrm{H}}$ defined in equation (2.1b), we find

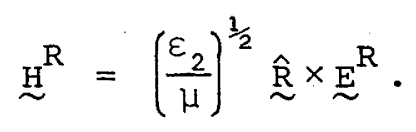

From equations (13) and (14), the time averaged Poynting

vector for the power in the radiation field, ${\underset{\sim}{R}}^{R}$, is in the radial, $\underset{N}{\mathbb{R}}$, direction and has the magnitude

$$
\begin{aligned}
S^{R} & =\frac{1}{2} \underset{\sim}{\mathbb{E}} \times{\underset{\sim}{H^{*}}}^{\stackrel{\hat{R}}{\sim}} \\
& =\frac{1}{2} \underset{\sim}{\mathbb{E}^{R}} \cdot \mathrm{E}^{R^{*}}
\end{aligned}
$$

which from equation (13) becomes

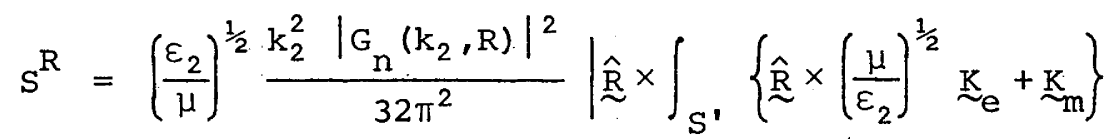

$$
\begin{aligned}
& \times\left.\exp \left(i k_{2} \underset{\sim}{\stackrel{\hat{R}}{\sim}} \cdot \mathbb{R}^{\prime}\right) d A^{\prime}\right|^{2} .
\end{aligned}
$$

This is the general form for the time averaged radiant energy flux for the power flowing away from the surface $S^{\prime}$. 


\author{
APPENDIX B \\ THE RADIATION FIELDS FROM AZIMUTHAL \\ TRAVELLING WAVE MODES AND THEIR RELATION \\ TO THE STANDING WAVE MODES
}

In Appendix A, we derived the general Surface Current Integral of the Surface Current Method. In this appendix, we use the modal fields of the weakly guiding dielectric optical fibre in the azimuthal travelling wave representation, i.e. $\exp \{ \pm i l \phi\}$ forms, to derive simpler expressions than equation (A.16) for the radiation fields. It is principally for these calculations that the modal fields were expressed in this travelling wave form. With these expressions, we then demonstrate their relations with the radiation fields of the standing wave modes.

In the azimuthal travelling wave form, the modal electric and magnetic fields are simply related via (equation (2.2.46))

$$
\underset{\sim}{\mathrm{H}}= \pm i \frac{|\ell|}{\ell}\left(\frac{\varepsilon_{2}}{\mu}\right)^{\frac{1}{2}} \underset{\sim}{\mathrm{E}},
$$

where the azimuthal variation is $\exp \{-i l \phi\}$. The + and - signs correspond to the $\mathrm{HE}_{\ell}$ and $\mathrm{EH}_{\ell}$ travelling wave modes respectively. We can obtain the more physically realistic standing wave modes, i.e. $\cos l \phi$ and $\sin l \phi$ form, by addition and subtraction of the $\pm l$ azimuthal travelling wave modes.

From equation (2.2) and equation (1) above

where

$$
\left(\frac{\mu}{\varepsilon_{2}}\right)^{\frac{1}{2}} \underset{\sim e}{\mathrm{~K}_{e}}=\mp i \frac{|l|}{\ell}{\underset{\sim m}{\mathrm{~m}}},
$$

$$
\underset{\sim m}{\mathbb{K}}=\underset{\sim}{\hat{n}} \times \underset{\sim}{\mathbb{E}}
$$


and from equations (A.13) and (A.16), using $G_{n}\left(k_{2}, R\right)=1 / R$ from equation (A.9), since the fibre is a three-dimensional structure,

$$
\stackrel{\mathrm{E}}{\mathrm{R}}^{\mathrm{R}}=\frac{i k_{2}}{4 \pi R} e^{-i k_{2} R}\left\{\underset{\sim}{\hat{\mathrm{R}}} \times\left(\mp i \frac{|l|}{\ell}(\underset{\sim}{\hat{\mathrm{R}}} \times \underset{\sim}{\Gamma})+\underset{\sim}{\Gamma}\right)\right\}
$$

and the time averaged Poynting vector $S^{R}$ is

$$
S^{R}=\left(\frac{\varepsilon_{2}}{\mu}\right)^{\frac{1}{2}} \frac{k_{2}^{2}}{32(\pi R)^{2}}\left\{\mid \underset{\sim}{\hat{R}} \times\left(\left.\mp \cdot i \frac{|\ell|}{\ell}(\underset{\sim}{\stackrel{R}{R} \times \underset{\sim}{\Gamma})+\Gamma)})\right|^{2}\right\},\right.
$$

where

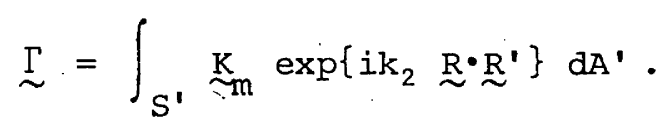

For the situations considered in this thesis, the circular symmetry of the optical fibre and of the azimuthal travelling wave modes will ensure that the radiation is azimuthally symmetric. Thus we need only evaluate the radiation field at one azimuthal position. For convenience, we choose this point to be $\phi=\pi / 2$, so that in the spherical co-ordinate system with the z-axis parallel to the fibre axis

$$
\underset{\sim}{\hat{R}}=\sin \theta \hat{y}+\cos \theta \underset{z}{\hat{z}}
$$

and using this in equation (5), we find

$$
S^{R}=\left(\frac{\varepsilon_{2}}{\mu}\right)^{\frac{1}{2}} \frac{k_{2}^{2}}{16(\pi R)^{2}}\left|\underset{\sim}{\Gamma} \cdot\left(\underset{\sim}{\hat{x}} \pm i \frac{|l|}{l}(\hat{y} \cos \theta-\hat{z} \sin \theta)\right)\right|^{2},
$$

where $\underset{\sim}{\hat{x}}, \hat{\mathrm{y}}, \hat{z}$ are the cartesian basis vectors as in fig. 2 . The • indicates the vector dot product.

From equation (5), we see that the form of the radiation from an azimuthal travelling wave mode is much simpler than the general form, equation (A.16). However, from the experience of the LP mode calculations of bending losses $[15,16]$ we must be very careful to ensure that the study of these travelling waves provides all the necessary information about the physically realistic standing wave modes. We shall now discuss their inter-relationship.

The azimuthal standing wave modes can be generated from the travelling wave formulation by addition and subtraction of the $\pm l$ modal 
fields, so that

$$
\left.\begin{array}{l}
\underset{S}{E_{C}}=\left(\mathbb{E}^{-} \mp \underset{\sim}{E^{+}}\right) \\
\underset{S}{\sim_{C}}=\left(\stackrel{H}{\sim}^{-} \underset{\sim}{H^{+}}\right)
\end{array}\right\}
$$

where the \pm superscripts refer to the $\pm l$ modes and the $c, s$ subscripts refer to the cos and sin modal fields. Using equation (1), equation becomes

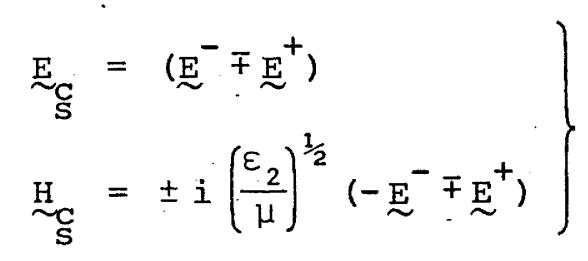

and the surface currents $\underset{\sim}{\mathrm{K}}$ and $\underset{\sim}{\mathrm{K}}$ for standing wave modes can be written as

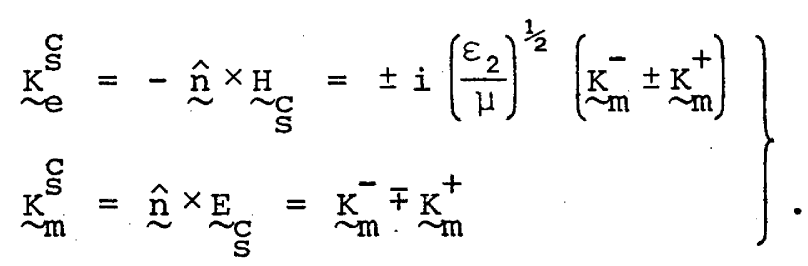

Thus, using equations (1) and equation (7) in equation (A.16), after much vector manipulation, the time averaged Poynting vector for the radiation field due to the standing wave modes reduces to

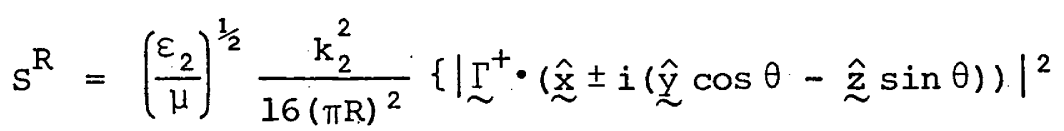

$$
\begin{aligned}
& \left.+\left|\Gamma_{\sim}^{-} \cdot(\underbrace{\hat{x}} \mp i(\underset{\sim}{\hat{y}} \cos \theta-\underset{\sim}{\hat{z}} \sin \theta))\right|^{2}\right\} .
\end{aligned}
$$

$I^{ \pm}$are defined in equation (6) with $\underset{\sim}{\mathrm{K}}=\underset{\sim}{\mathrm{K}}=\underset{\sim}{\hat{n}} \times \underset{\sim}{ \pm}$. The \pm signs refer to the $\mathrm{HE}$ and $\mathrm{EH}$ modes, respectively. From equation (12) one can see that there is no difference between the radiated power from the sin $\ell \phi$ and $\cos \ell \phi$ modes from equation (12). The total power radiated from a standing wave mode is the sum of the powers radiated by each of its component travelling wave modes. Thus, there is no interference between the radiation from the two travelling wave modes.

Upon the realisation that the normal to the core-cladding 
interface is $\underset{\mathfrak{r}}{\hat{\sim}}$, and using the core-cladding interface as the surface for the integrals, we have

where

$$
\underset{\mathrm{m}}{\mathrm{K}_{\mathrm{m}}^{ \pm}}=-\underset{\mathrm{z}}{\mathrm{E}^{ \pm}} \stackrel{\Phi}{\sim}+\mathrm{E}_{\phi}^{ \pm} \underset{\mathrm{z}}{\sim}
$$

$$
\hat{\phi}=-\sin \phi^{\prime} \underset{\sim}{\hat{x}}+\cos \phi^{\prime} \underset{\sim}{\hat{y}} \text {. }
$$

Upon using the relations between $\mathrm{E}_{\phi}^{+}$and $\mathrm{E}_{\phi}^{-}$and those between $\mathrm{E}_{\mathrm{z}}^{+}$and $\mathrm{E}_{\mathrm{z}^{-}}^{-}$ and transforming the integral $\phi^{\prime}$ from $\phi^{\prime}$ to $\left(\pi-\phi^{\prime}\right)$ for the $(-l)$ modes, we find that for surfaces which, for the radiation field at $\phi=\pi / 2$, are such that

$$
\underset{\sim}{\stackrel{R}{R} \cdot \mathbb{R}^{\prime}}\left(\pi-\phi^{\prime}\right) \equiv \underset{\sim}{\mathbb{R}} \cdot \mathbb{R}^{\prime}\left(\phi^{\prime}\right)
$$

we have, using equations (13) and (16),

$$
\left|{\underset{\sim}{\Gamma}}^{-} \cdot(\underset{\sim}{\hat{x}} \mp i(\underset{\sim}{\hat{y}} \cos \theta-\underset{\sim}{\hat{z}} \sin \theta))\right| \equiv \mid \stackrel{\sim}{\Gamma}^{+} \cdot(\underset{\sim}{x} \pm i(\underline{\underline{z}} \cos \theta-\hat{z} \sin \theta) \mid \cdot
$$

Equation (15) is a statement that for radiation losses that are symmetric in the azimuthal direction satisfying equation (14), the $\pm \ell$ azimuthal travelling wave modes radiate equally. Thus, for such situations, using equation (15) in equation (12), the radiation loss of the azimuthal standing wave modes is twice the radiation loss of either of its component azimuthal travelling wave modes.

From equations (12) and (15), we have derived two important results, viz.:

(1) Each component azimuthal travelling wave mode of an azimuthal standing wave mode radiates independently.

(2) The magnitude of the radiation from each of the travelling wave modes is equal. 


\section{APPENDIX C \\ RADIATION FROM THE MODES OF THE IDEAL STRAIGHT DIELECTRIC OPTICAL FIBRE: \\ THE TUNNELLING LEAKY MODES}

From Appendix B, we know that we only need to analyse one of the azimuthal travelling wave modes. For convenience, we choose the mode with $\exp \{-i l \phi\}$ variation, i.e. $l \geqslant 0$ mode. Thus, the formula for the time averaged poynting vector of the radiation field, from equation (B.5) and (B.6), is

$$
S^{R}=\left(\frac{\varepsilon_{2}}{\mu}\right)^{\frac{1}{2}} \frac{k_{2}^{2}}{32(\pi R)^{2}}\left\{\mid \Gamma \cdot\left(\hat{x} \pm\left. i(\hat{z} \cos \theta-\hat{z} \sin \theta)\right|^{2}\right\},\right.
$$

where the + and - signs refer to the HE and EH modes respectively, and

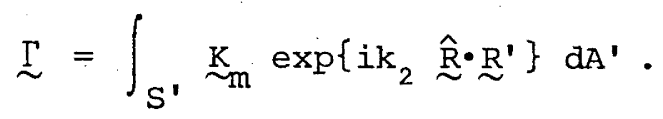

We are going to calculate the radiation loss of the modes of the ideal dielectric optical fibre, including both "improper" and "proper" modes. In this analysis, we choose a finite length of fibre, length $2 L$, centred about the origin of the spherical and cylindrical co-ordinate systems, as in fig. 3. Although the surface $S^{\prime}$ over which equation (2) is evaluated is quite arbitrary, we choose the corecladding interface for which the unit normal to the surface is $\underset{\mathfrak{x}}{\hat{\sim}}$. Thus

$$
\begin{aligned}
\underset{\sim m}{K} & =\stackrel{\hat{\sim}}{\sim} \times{\underset{\sim}{S}}_{S} \\
& =-E_{z}\left\{-\sin \phi^{\prime} \underset{\sim}{\hat{x}}+\cos \phi^{\prime} \hat{y}\right\}+E_{\phi} \hat{z},
\end{aligned}
$$

where $\mathrm{E}_{z}$ and $\mathrm{E}_{\phi}$ are evaluated at the core-cladding interface. From equations $(2.2 .43)-(2.2 .48)$, the normalised fields at the core-cladding 
boundary are

and

$$
\left.\begin{array}{l}
E_{\phi}=A_{\ell \mp 1} \exp \{-i(\ell \phi+\beta z)\} \\
E_{z}=-A_{\ell \mp 1} B_{\ell \mp 1} \exp \{-i(\ell \phi+\beta z)\}
\end{array}\right\},
$$

where from equations $(2.2 .43)-(2.2 .48)$

$$
B_{\ell \mp 1}=\frac{i W}{k_{2} \rho} \frac{H^{(I)}(i W)}{H_{l \mp 1}^{(1)}(i W)}
$$

and $A_{\ell \mp I}$, defined in equation (2.2.53), is the power normalisation coefficient to ensure unit power flow in the mode.

Using equation (4) in equation (3)

$$
\left.\underset{\sim}{\mathrm{K}}={ }^{A_{\ell \mp 1}}{ }^{B_{\ell \mp 1}}\left(-\sin \phi^{\prime} \underset{\sim}{\hat{x}}+\cos \phi^{\prime} \underset{\sim}{\hat{y}}\right)+\underset{\sim}{\hat{z}}\right\} \cdot \exp \left\{-i\left(\ell \phi^{\prime}+B z\right)\right\} .
$$

Due to the symmetry of the mode, the radiation will be symmetric azimuthally so that the total power need only be evaluated at one position $\phi=\pi / 2$, in the radiation field, and (see fig. 2)

$$
k_{2} \underset{\sim}{\stackrel{R}{\sim}} \cdot \mathbb{\sim}^{\prime}=k_{2} \rho \sin \theta \sin \phi^{\prime}+k_{2} z^{\prime} \cos \theta \text {. }
$$

Thus equation (2) becomes

$$
\begin{array}{r}
\stackrel{\Gamma}{\sim} \mathrm{A}_{\ell \mp 1} \rho \int_{0}^{2 \pi} \mathrm{d} \phi^{\prime} \int_{-L}^{L} \mathrm{~d} z^{\prime}\left(\exp \left\{i \cdot\left[k_{2} \rho \sin \theta \sin \phi^{\prime}+\left(k_{2} \cos \theta-\beta\right) z^{\prime}-\ell \phi^{\prime}\right]\right\}\right. \\
\quad \times\left(-B_{\ell \mp 1} \sin \phi^{\prime} \underset{\sim}{\hat{x}}+B_{\ell \mp 1} \cos \phi^{\prime} \hat{\underline{z}}+{\underset{\sim}{\hat{z}})}^{\hat{~}}\right) .
\end{array}
$$

The integration of $z^{\prime}$ is straightforward, and upon recognition of the integral representation of the Bessel function of the first kind [17], viz.

$$
2 \pi J_{n}(x)=\int_{0}^{2 \pi} \exp \left\{i x \sin \phi^{\prime}-i l \phi^{\prime}\right\} d \phi^{\prime} .
$$

The $\phi^{\prime}$ integral can be done so that equation (7) becomes

$$
\underset{\sim}{\Gamma}=A_{\ell \mp 1} 4 \pi \rho \frac{\sin \left(k_{2} \gamma L\right)}{k_{2} \gamma}\left(i_{\ell \mp 1} J_{\ell}^{\prime}(\ell) \underset{\sim}{\hat{x}}+\frac{B_{\ell \mp 1} \ell}{Q} J_{\ell}(\ell) \underset{\sim}{\hat{y}}+J_{\ell}(Q) \underset{\sim}{\hat{z}}\right),
$$


where the argument of the Bessel functions, $Q$,

$$
\begin{aligned}
Q & =k_{2} \rho \sin \theta \\
k_{2} \gamma & =k_{2} \cos \theta-\beta .
\end{aligned}
$$

The ' indicates differentiation with respect to the argument $Q$. Then using equation (9) in equation ( 1 ), the time averaged Poynting vector becomes

where

$$
S^{\dot{R}}=G(\theta) \frac{\sin ^{2}\left(k_{2} \gamma L\right)}{\gamma^{2}},
$$

$G(\theta)=\left(\frac{\varepsilon_{2}}{\mu}\right)^{\frac{1}{2}} \frac{\left(k_{2} \rho\right)^{2}}{\left(k_{2} R\right)^{2}}\left(A_{\ell \mp 1}\right)^{2}\left\{{ }_{l \mp 1} J_{\ell}^{\prime}(Q) \pm J_{\ell}(Q)\left(\frac{B_{\ell \mp 1} \cdot \ell \cos \theta}{Q}-\sin \theta\right)\right\}^{2} \cdot$

The total power radiated, $\mathrm{P}^{\mathrm{R}}$, is

$$
P^{R}=2 \pi R^{2} \int_{0}^{\pi} S^{R} \cdot \sin \theta d \theta
$$

and thus, using equation (12), this becomes

$$
P^{R}=2 \pi R^{2} \int_{0}^{\pi} G(\theta) \frac{\sin ^{2}\left(k_{2} \gamma L\right)}{\gamma^{2}} \theta d \theta
$$

Changing variables in equation (15) via

$$
\mathbf{x}=\gamma
$$

so that

$$
d x=-\sin \theta d \theta
$$

and using the integral [19], where a $>0$

$$
\int_{-a}^{b} G(x) \frac{\sin ^{2}(p x)}{x^{2}} d x=G(0) \cdot p \pi+o\left(\frac{1}{p}\right)
$$

we have that if

$$
\gamma=0
$$

over the range of angles $\theta=0$ to $\pi$, then for $k_{2} L \gg>1$ in equation (15), significant radiation loss occurs. From equations (10) and (18), the radiation loss is significant if 


$$
\cos \theta_{0}=\frac{\beta}{k_{2}} \leqslant 1
$$

Since the bound, proper modes have $\beta>k_{2}$, they cannot contribute significant radiation losses. However, for those modes with $\beta<k_{2}$, i.e. the tunnelling leaky modes and the refracting modes, the radiation loss is significant and is predominantly at the angle $\theta_{0}$, defined in equation (19), to the axis.

In this case, the radiative power loss $\mathrm{P}^{\mathrm{R}}$ from the mode, using equations (12) - (19), becomes

$$
\mathrm{P}^{\mathrm{R}}=2 L\left(\frac{\varepsilon_{2}}{\mu}\right)^{\frac{1}{2}} \pi^{2} \mathrm{k}_{2} \rho^{2}\left(\mathrm{~A}_{\ell \mp 1}\right)^{2}\left\{\mathrm{~B}_{\ell \mp 1} \mathrm{~J}_{\ell}^{\prime} \pm J_{\ell}\left(\frac{\mathrm{B}_{\ell \mp 1} l \beta}{\ell}-\frac{Q}{\mathrm{k}_{2} \rho}\right)\right\}^{2},
$$

where

$$
\begin{aligned}
Q & =k_{2} \rho \sin \theta_{0} \\
& =\rho\left(k_{2}^{2}-\beta^{2}\right)^{\frac{1}{2}} .
\end{aligned}
$$

Using the recurrence relations for Bessel functions [17], the forward directed radiation for which $\beta / k_{2} \sim 1$

$$
\mathrm{P}^{\mathrm{R}}=2 \mathrm{~L}\left(\frac{\varepsilon_{2}}{\mu}\right)^{\frac{1}{2}} \pi^{2} \mathrm{k}_{2} \rho^{2} \mathrm{~A}_{\ell \mp 1}^{2}\left(\mathrm{~B}_{\ell \mp 1} \mathrm{~J}_{\ell \mp 1}(Q)-\frac{Q}{\mathrm{k}_{2} \rho} \mathrm{J}_{\ell}(Q)\right)^{2} .
$$

The power attenuation coefficient of the leaky mode then becomes

$$
\alpha=\frac{P^{R}}{2 L}
$$

since the incident mode was normalised to unit incident power. Therefore, equation (23) becomes

$$
\alpha=\left(\frac{\varepsilon_{2}}{\mu}\right)^{\frac{1}{2}} \pi^{2} k_{2} \rho^{2} A_{\ell \mp 1}^{2}\left(B_{\ell \mp 1} J_{\ell \mp 1}(Q)-\frac{Q}{k_{2} \rho} J_{\ell}(Q)\right)^{2} .
$$

Now, from the analytic continuation of the field expressions of the bound modes below cutoff via replacing $i w$ by $Q$ where

$$
Q=\rho\left(k_{2}^{2}-\beta^{2}\right)
$$

we find, using the Wronskian relations for Bessel functions [17] of the 
first kind, and equation (5) for $\mathrm{B}_{\ell \mp l}$, that equation (24) becomes

$$
\alpha=\frac{4}{k_{2}}\left(\frac{\varepsilon_{2}}{\mu}\right)^{\frac{1}{2}}\left(A_{\ell \mp 1}\right)^{2}\left(H_{\ell \mp 1}^{(1)}(Q)\right)^{-2} \text {. }
$$

For step-index waveguides for which, by analytic continuation of equation (2.3.12), we have

$$
\left(A_{\ell \mp I}\right)^{2}=\left(\frac{\varepsilon_{2}}{\mu}\right)^{\frac{1}{2}} \pi \rho^{2}\left(\frac{V}{U}\right)^{2} \frac{H_{\ell \mp 2}^{(I)}(Q) H_{\ell}^{(1)}(Q)}{\left(H_{\ell \mp I}^{(1)}(Q)\right)^{2}} .
$$

Then the power attenuation coefficient of the tunnelling leaky modes of the step-index dielectric optical fibre is

$$
\alpha_{\text {S.I. }}=\frac{4}{\pi} \frac{U^{2}}{k_{2} \rho^{2} V^{2}}\left(H_{\ell \mp 2}^{(1)}(Q) H_{\ell}^{(1)}(Q)\right)^{-1},
$$

where

$$
\begin{aligned}
Q & =k_{2} \rho \sin \theta_{0} \\
& =\rho\left(k_{2}^{2}-\beta^{2}\right)^{\frac{1}{2}} .
\end{aligned}
$$

This agrees with the well known power attenuation coefficient reported by Snyder and Mitchell $[2,3,18]$. 


\section{REFERENCES}

[1] A.W. Snyder and D.J. Mitchell: "Leaky mode analysis of circular optical waveguides", Opto-Electronics $\underline{6}, 287$ (1974).

[2] A.W. Snyder and D.J. Mitchell: "Leaky rays cause failure of geometric optics on optical fibres", Electron. Letters 9, 457 (1973).

[3] Rayleigh, Lord: "Further applications of Bessel functions of higher order to the whispering gallery and allied problems", Phil. Mag. 27, 100 (1914).

[4] R.D. Richtmeyer: "Dielectric resonators", J. Appl. Phys. 10, 39 (1939).

[5] L.D. Landau and E.M. Lifshitz: Quantum Mechanics (Pergamon, Oxford, 1965).

[6] E.A.J. Marcatili: "Bends in optical dielectric guides", B.S.T.J. 48, 2103 (1969).

[7] M.A. Miller and V.I. Talanov: "Electromagnetic surface waves guided by small curvature boundaries", Wh. Tekh. Fiz. 26, 2755 (1956).

[8] V.V. Shevchenko: "Radiation losses in bent waveguides for surface waves", Izv. VWZ Radiofizika 14, 768 (1971).

[9] E.A.J. Marcatili: "Dielectric guide with curved axis and truncated parabolic index", B.S.T.J. 49, 1645 (1970).

[10] A.W. Snyder and D.J. Mitchell: "Generalised Fresnel's laws for determining radiation loss from optical waveguides and curved dielectric structures", Optik $\underline{40}, 438$ (1974).

[11] S.A. Schelkunoff: Electromagnetic Waves (Van Nostrand, 1943), p. 158 .

[12] J.A. Stratton: Electromagnetic Theory (McGraw-Hill, New York, 1941), p.464.

[13] Ref. 12, p. 460.

[14] P.M. Morse and H. Feshbach: Methods of Theoretical Physics, Vol. 1, Chapt. 7 (McGraw-Hill, New York, 1953). 
[15] D. Marcuse: "Curvature loss formula for optical fibers", J. Opt. Soc. Am. 66, 216 (1976).

[16] D.C. Chang and E.F. Kuester: "General theory of surface wave propagation on a curved optical waveguide of arbitrary crosssection", Scientific Report No. 11, Electromagnetics Lab., Dept. Elect. Eng., Univ of Colorado, Boulder, Colorado (including the supplement to this report). The results are also reported in I.E.E.E. J. Quant. Electron. QE-11, 903 (1975).

[17] M. Abramowitz and I.A. Stegun: Handbook of Mathematical Functions (Dover; New York, 1973), Chapt. 9.

[18] A.W. Snyder and D.J. Mitchell: "Ray attenuation in lossless dielectric structures", J. Opt. Soc. Am. 64, 956 (1974).

[19] A.W. Snyder: "Radiation loss due to variations of radius of dielectric or optical fibers", I.E.E.E. Trans. MTT-18, 608 (1970). [See the appendix to this paper.)

[20] E.G. Rawson: "Analysis of scattering from fiber waveguides with irregular core surfaces", Appl. Optics 13, 2270 (1974).

[21] R.A. Abram and C.J. Rees: "Mode conversion in an imperfect waveguide", J. Phys. A 6 , 1693 (1973). 


\section{CHAPTER 6 \\ RADIATION FROM BENDS IN OPTICAL WAVEGUIDES}

\subsection{INTRODUCTION}

In the previous chapter, we introduced the surface Current Method and applied the formulation to the determination of the attenuation coefficients of the tunnelling leaky modes of the dielectric optical fibre of circular cross section. In this chapter, the method is applied to the calculation of the radiative losses of bent dielectric waveguides. This study is motivated by the presence of such bends in the practical optical communications system, in which the fibres must be bent to accommodate natural obstructions. The susceptibility of the dielectric optical waveguide to bending losses will play a major rôle in the design of practical optical waveguide characteristics.

From the heuristic description of the physical mechanism of curvature induced radiation presented in Chapter 5, we can determine qualitatively which fibre parameters significantly affect the propagation characteristics of the optical fibre with bends in the waveguide axis. In the discussion of Chapter 5, the factors that significantly pre-determine the susceptibility of an electromagnetic wave to curvature losses were:

(1) The fraction of power of the electromagnetic wave guided in the cladding.

(2) The distribution of this energy over the cladding region.

In optical waveguides, the fibre parameters characterising these two factors for a mode are $(1-n)$, where $n$ is the fraction of the total guided power of the mode [1] that travels within the core, and the transverse decay constant of the fields in the cladding, $w / \rho$ where 


$$
W=\rho\left(\beta^{2}-k_{2}^{2}\right)^{\frac{1}{2}}
$$

where $\beta$ is the propagation constant of the mode and $k_{2}$ is the wavenumber in the cladding.

The fraction of power travelling in the cladding, $(1-n)$, is a function of the normalised frequency of the optical fibre, $V$, where

and

$$
\mathrm{V}=\mathrm{k}_{\mathrm{l}} \rho \theta_{\mathrm{C}}
$$

$$
\theta_{c}=\left(1-\frac{\varepsilon_{2}}{\varepsilon_{1}}\right)^{\frac{1}{2}} \ll 1 \text {, }
$$

where $k_{1}$ is the wavenumber in the core and $\rho$ is the radius of the core. The fraction, $1-n$, increases for a mode as $v$ decreases, i.e. as the mode approaches cutoff. Thus, as a mode approaches cutoff, the susceptibility of the mode to curvature losses increases.

In an optical fibre $W$ is a function of $V$ but $W / P$ can vary independently of $V$, so that as $w / p$ decreases the field in the cladding becomes less confined to the core region, and the modal susceptibility to bending losses increases. W/ $p$ can be decreased by decreasing the corecladding dielectric difference as in this case $k_{2} \rightarrow k_{1}$ and $\left|k_{2}-\beta\right| \rightarrow 0$.

Thus we have determined the two critical factors that strongly affect the susceptibility of a mode to bending losses:

(1) The normalised frequency of the fibre, $V$; and

(2) The dielectric difference between the core and cladding dielectrics, or equivalently the acceptance angle of the fibre, $\theta_{c}$.

However, we have not, as yet, discussed the effect of reducing the radius of curvature on the magnitude of these losses. If we consider the wave in fig. 1, the local velocity of propagation of the rotating wave travelling around the interface at a distance $R$ from the centre of curvature is

$$
v_{\mathrm{R}}=\frac{\omega \mathrm{R}}{\mathrm{v}}
$$

where $\nu$ is the mode number of the azimuthal phase variation around the 


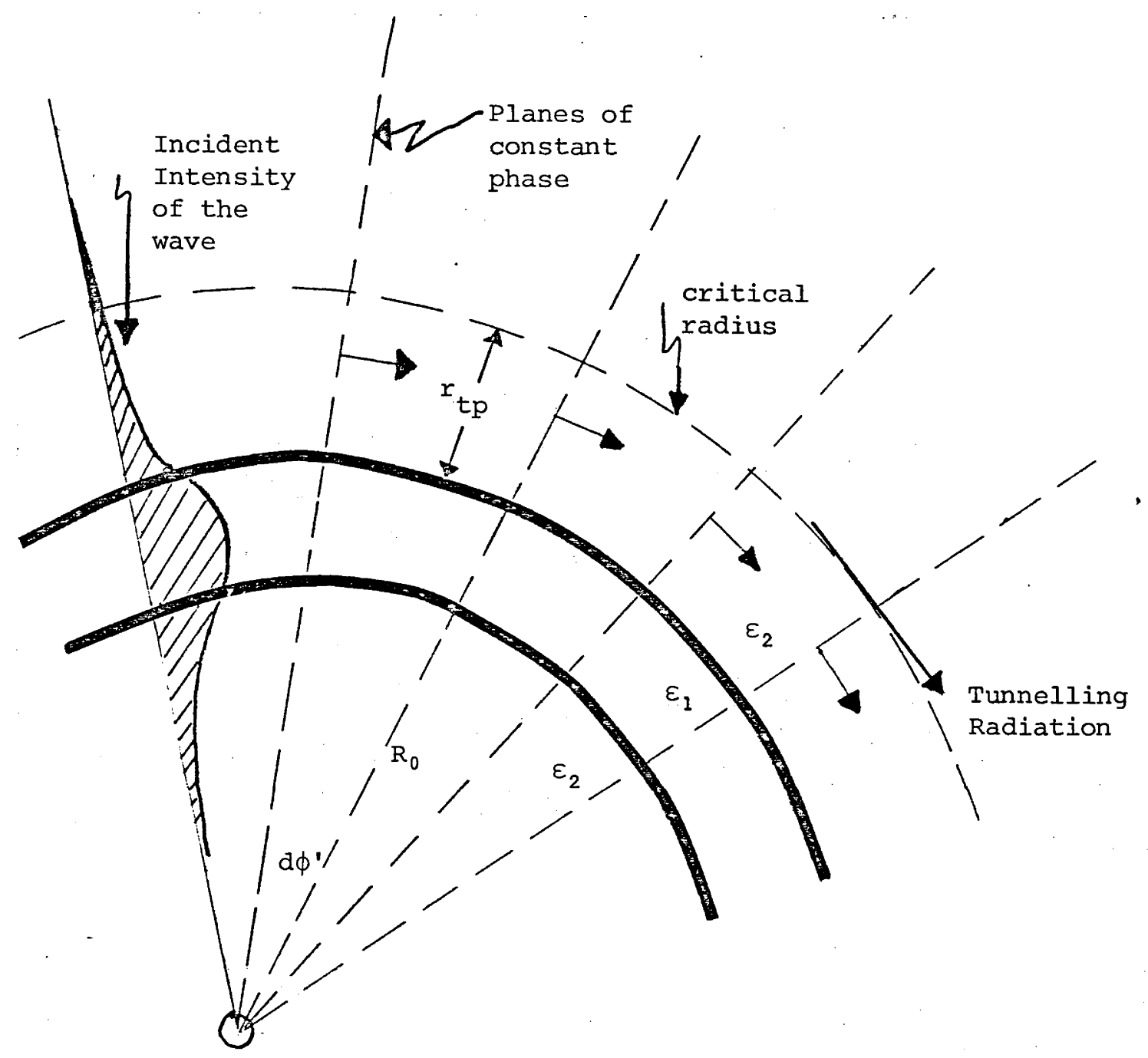

Fig. 1: Propagation of an electromagnetic wave around a bend in the fibre axis. 
interface, expressed as $\exp \{-i \ell \phi\}$, and matching this phase variation on the axis of the waveguide to that of the straight waveguide:

$$
\nu=\beta R_{0}
$$

where $\beta$ is the axial phase propagation constant of the wave in the straight waveguide.

At a distance $R^{\prime}$, from the centre of curvature, this propagation velocity equals the velocity of propagation of light in the cladding and from beyond this point the wave can detach itself from the guided wave; thus, this distance $R^{\prime}$ at which the radiation re-appears as a travelling wave is

$$
\frac{\omega R^{\prime}}{\beta R_{0}}=\frac{\omega}{k_{2}}
$$

from which we find

$$
\left(R^{\prime}-R_{0}\right)=\left(\frac{\beta}{k_{2}}-I\right) R_{0}
$$

Energy beyond $R^{\prime}$ ultimately radiates away from the core-cladding interface, so that the distance $\left(R^{\prime}-R_{0}\right)$ will cxitically determine the magnitude of the radiation loss due to the curvature. As $\left(R^{\prime}-R_{0}\right)$ decreases, more of the power in the cladding will lie beyond this point, the "caustic" or "turning point" mentioned previously, and hence the amount of radiation from the mode will increase. Therefore as $R_{0}$ decreases, $\left(R^{\prime}-R_{0}\right)\left(=r_{t p}\right.$ in fig. 1) decreases, and the radiative losses will increase. The radius of curvature, $R_{0}$, of the axis of the waveguide then forms the third critical parameter, together with the two aforementioned modal parameters, that determine the susceptibility of a mode to bending losses.

Throughout this discussion and in the ensuing analysis we do not consider the effect of the bend on the coupling of the incident mode to other bound modes of the straight optical fibre. The justification for ignoring this analysis is twofold. As in Chapters 3 and 4, we only derive the radiative losses from a mode since Coupled Mode Theory can be easily applied to determine the magnitude of the bound mode coupling, ${ }^{+}$

+ We require the adiabatic Coupled Mode Theory, i.e. the Local Normal Mode formulation, to analyse the mode coupling in a bend [2-3] 
but cannot be extended to treat the coupling to the radiation modes as discussed in Chapter 5. Secondly, from our heuristic description of the bending loss we observe that the magnitude of the radiative losses is critically dependent on the magnitude of $\mathrm{V}$. As $\mathrm{V}$ increases most modes, except the modes near cutoff, become less susceptible to bending losses due to radiation. Thus, although the analysis of this chapter is general, our discussions are confined to monomode waveguides for which only radiative losses are extant.

Now that our heuristic description of the bending loss due to radiation has allowed us to isolate the significant fibre parameters, we shall discuss the different approaches to the quantitative analysis of this loss.

Until recently, the radiative losses of modes of a straight optical waveguide of finite cross section incident on a bend in the waveguide, has not been analysed with any authority [4-20]. Until this time, approximate analyses by a wide range of techniques were only applicable to planar (slab) waveguides [21-26] which were unphysical models due to the infinite extent of the cross section of the waveguide. The results of these analyses of the two-dimensional waveguides were often extrapolated to the three-dimensional waveguides of finite cross section, in an attempt to derive order of magnitude estimates of the radiative losses of the modes of the waveguides of finite cross section. Although such studies provide physical insight into the mechanism of curvature induced losses, experimental results indicate that they do not provide quantitatively accurate results [27], although their dominant exponential behaviour is the same.

In general, the theoretical studies have been primarily concerned with radiative losses due to constant curvature of the waveguide, i.e. the radiation that Chang and Kuester refer to as the continuous radiation [28]. Only a few analyses have attempted to answer the question of the effect of variations in the curvature of the waveguide axis [28-30]. However, these analyses are based on models which do not appear to be physically realistic since they consider discontinuous changes in the radius of curvature ${ }^{\dagger}$ of the bent waveguide,

+ Such discontinuous variations in the radius of curvature would enhance the effect of variations in curvature considerably. 
that do not occur in practical optical fibres. In the following analysis, we shall only consider the "continuous radiation" due to constant curvature of the waveguide axis.

The exact analysis of the radiative losses from bent optical waveguides requires the knowledge of the electromagnetic fields of the bent waveguide, and their associated power attenuation coefficients, ${ }^{\dagger}$ and the magnitude of the respective coupling coefficients of these modes to the modes of the straight waveguide. From this information, one can readily determine the effect of the bend on propagation of an incident mode of the straight waveguide. However, for optical waveguides of finite cross section, this requires the knowledge of the solutions of the vector Helmholtz wave equation in toroidal co-ordinates. Unfortunately, such solutions do not exist in a form useful for calculations and even for the scalar wave equation in toroidal co-ordinates in which solutions exist in the form of an infinite series of Legendre polynomials, they suffer from the further disadvantage of being a non-orthogonal set of modes [32].

It is therefore necessary to seek approximate solutions to these problems. In the past few years, several different approaches have been developed for waveguides of finite cross section. We shall now list these techniques and briefly summarise the philosophy of each approach.

(a) Cladding Energy Considerations [4-6,21]: This analysis is, basically, the quantitative extension of the heuristic discussion presented earlier. The energy travelling beyond the turning point is determined and assumptions are made as to the rate at which this energy is radiated from the waveguide core. Although relatively simple in application to twodimensional waveguides, the analysis proves less tractable for finite cross-sectioned waveguides.

(b) Ray Optical Analysis $[18,19,22]$ : The generalised Fresnel's Law $^{*}$ [19] for rays incident at a curved interface between

+ From a fundamental theory of electromagnetism [ ], no closed structure can support pure bound unattenuated modes. As a toroidal structure is a closed structure, all "modes" must radiate to a degree.

* See bottom of p. 197 . 
two dielectrics is used to determine the attenuation coefficient of the ray. This technique has been successfully applied to the two-dimensional slab waveguides but as yet has not been applied to the bent fibre, although such an extension appears possible. Strictly, such a ray optical analysis should be restricted to multimode fibres.

(c) Conformal Mapping $[22,20]$ : By conformal mapping of the bent optical waveguide into a straight optical waveguide with an induced dielectric profile representing the effect of the bend, energy considerations as in (a) can be employed to determine the energy beyond the caustic and hence the radiative loss. Such an analysis is particularly suited to two-dimensional waveguides but appears to be of little significance for waveguides of finite cross section due to the complexity of the conformal mapping.

(d) Spectral Representations of the Fields of the Bent Waveguide [7-10,12-17]: The field of the bent waveguide is constructed from a spectrum of waves of a complete mode set. With this representation, the radiation fields of the bent fibre can be constructed and conventional excitation formulae of electromagnetic theory [33] can be used to determine the coupling into these fields. While some of these spectral representations have physical significance, i.e. cylindrical waves, plane waves, etc., others have been ingeniously, and one suspects laboriously constructed by inspired trial and error, searching for suitable representations. This approach has the advantage that it is theoretically an exact treatment.

(e) The Surface Current Method [19]: This approach employs the Equivalence Principle [34] formulated by Schelkunoff, to determine the radiation from a toroid, from the knowledge of the fields over the surface of the bent waveguide. This analysis removes the necessity for the spectral representation of the radiation fields of the bent structure, and as with (d) above has the capacity to be an exact treatment.

* This can be considered as a more formalistic treatment of the energy considerations of approach (a). [This notes refers to bottom of p.196.] 
of the above techniques, the latter two, (d) and (e), have the most general application, together with the capacity to provide an exact analysis for all dielectric optical waveguides. The question as to the relative accuracy of the perturbation analyses using either approach is open. It is very difficult to go beyond the lowest order perturbation solutions by both approaches, and as well it is difficult to correlate the two perturbation analyses, although the results agree exactly.

Two recent studies $[15,13]$ on the calculation of bending losses in dielectric optical waveguides have provided valuable insight into the processes occurring and the approximations employed in the analysis. Chang and Kuester's study $[15,16]$ of the radiation losses, using a spectral representation of the field have provided the extremely important interpretation of the phase matching procedure by which the axial phase variation of the straight fibre mode is converted to a phase change around the bent waveguide. In their study, the authors demonstrate that this phase matching procedure is equivalent to matching the phase of the modes along the path of the "centre of energy" of the mode, the electromagnetic analogue of the centre of mass of a system of particles in classical mechanics. Before this study, the choice of the path along which this matching procedure was applied, was quite arbitrary and led to results which were in disagreement by orders of magnitude for multimode waveguides $[10,15]$. In general, the path was chosen by the "obvious" choice of the axis of the straight waveguide. However, for asymmetric slab waveguides, Chang and Kuester's study [16] demonstrates that significant errors can be introduced by this choice.

In a recent article, Marcuse [13] has determined the effect of the deformation of the incident modal field in the bend of the waveguide by a numerical analysis. Most studies, implicitly or explicitly, consider the incident modal field of the straight waveguide to propagate without deformation around the bend. Although this physically would appear to be correct for some regions of the bent waveguide, at least for a sufficiently large radius of curvature, until this time no quantitative results were available as to the magnitude of the bending radius for which this approximation was justified. We shall return to these points in sections 4 and 5 . 
In section 2, we discuss the philosophy of the Surface Current Method for the bending losses in optical waveguides. In section 3, we re-derive the well known result of the bending loss of the symmetric slab waveguide by the Surface Current Method and compare this result with the ray optical analysis employing the generalised Fresnel's law [19]. This section is presented in order to clarify some of the approximations used in the Surface Current Method and common to all calculations on bending losses.

In section 4, we present the calculations of the radiative loss from a bent optical fibre of circular cross section, using the Surface Current Method. In the calculations, we employ the general modal formulation of chapter 2. In section 5, we use a ray optical analysis on slab waveguides to determine order of magnitude estimates of the bending radius for which field deformation and mode coupling are not significant. We discuss the applicability of these results to the bent optical fibre.

Finally, in section 5, we discuss the applicability of these results for practical optical fibres and consider the effects that have been neglected in the analysis.

\subsection{THE PHILOSOPHY OF THE SURFACE CURRENT METHOD FOR THE ANALYSIS OF BENDS IN OPTICAL WAVEGUIDES}

In Chapter 5, the formulation of the Surface Current Method was presented. In that presentation, it was emphasised that the analysis is mathematically exact if the electromagnetic fields over the core-cladding interface are known exactly. However, in the bends in optical waveguides, the electromagnetic fields are not known exactly due to the complexity of the wave equation, as discussed previously. We therefore need to be able to approximate these fields in the bent waveguide.

Physically, if the bend in the axis of the waveguide is very slow, then, locally, near the core of the waveguide, every finite length of the waveguide, dz', appears to be locally straight, and the fields 
should be approximately the same shape and have the same field intensity distribution as the straight optical waveguide. ${ }^{\dagger}$ In our attempt to approximate the fields near the core the bent waveguide of constant curvature radius, $R_{0}$, we must also convert the axial phase variation of the straight fibre mode, i.e. $\exp \{-i \beta z\}$ to a phase variation around the circular bend of radius, $R_{0}$, i.e. $\exp \{-i m \phi\}$ as in fig. 1. Considering an elemental length $\mathrm{dz}$ ' of the bent waveguide, and requiring that the phase variation along the axis of the locally straight segment* be equal to the phase variation of the field around the bend, we find

$$
\beta d z^{\prime}=m d \phi^{\prime}
$$

and from $d z^{\prime}=R_{0} d \phi^{\prime}$, we find

$$
\beta R_{0}=m,
$$

where $\mathrm{m}$ is the modal number of the bent waveguide around the waveguide axis.

Thus, assuming the field distribution in the bent waveguide of constant radius of curvature of the axis, $R_{0}$, is the same as the distribution of the incident mode, near the core of the waveguide, and using equations (1): and (2) to describe the propagation of this field distribution around the bend, we have a suitable approximation for the field in the bent waveguide. This is the field that we shall use in the Surface Current Method calculations. Obviously, the field will become more deformed the further from the core, since at some position it will no longer be an evanescent wave. Thus, we choose the core-cladding interface as the surface over which the Surface Current Method is to be applied.

The analysis of the bends in dielectric optical waveguides by the Surface Current Method using the approximations above, cannot be justified except by physical intuition. Although the analyses of

+ From this argument we cannot provide a quantitative restriction on the magnitude of the bending radius for which this statement applies. We discuss this in section 5.

* Since we will consider only symmetric waveguides in this thesis, the axis of the waveguide is the path along which the phase matching should be accomplished [35]. 
bending loss by the spectral field representation have the capacity to be exact treatments all employ, explicitly or implicitly, the above approximations; however, it is difficult to discern the introduction of these approximations in some analyses, e.g. Lewin's analysis $[7,8]$ and Chang and Kuester's analysis $[15,16]$ purport to include the first order corrections to the incident fields. However, without these first order corrections, these analyses produce the trivial result of zero radiation. It is difficult to determine whether the first order corrections to the fields included in these analyses correspond to the zeroth order analyses of other authors [9-13], but it is the opinion of the author that they do.

Now that we have presented the philosophy of the Surface Current Method in the calculations of radiative losses from bends in optical waveguides, let us discuss more fully the mechanics of the calculations. In the following analysis, we restrict ourselves to the radiative losses due to complete bends of constant radius of curvature $R_{0}$, so that we isolate the curvature effect from all other radiation mechanisms. Thus, for two-dimensional waveguides, we consider the complete annulus, as in fig. $2 a$, and for the bent optical waveguide, we consider the complete torus of circular cross section, as in fig. $2 \mathrm{~b}$. The exclusion of the end effects due to a finite bend will not affect attenuation coefficient of the bent optical fibre, as the radiated power from the variation in radius of curvature at the ends of the finite bend are not strongly length dependent effects. ${ }^{\dagger}$

Now that we have presented the philosophy of the Surface Current Method for bent optical waveguides of constant curvature, we shall present the two examples mentioned above, i.e. the bent slab and the bent optical fibre.

The length dependence of such variations is purely due to the interference between the radiation from either end of the finite bend. 


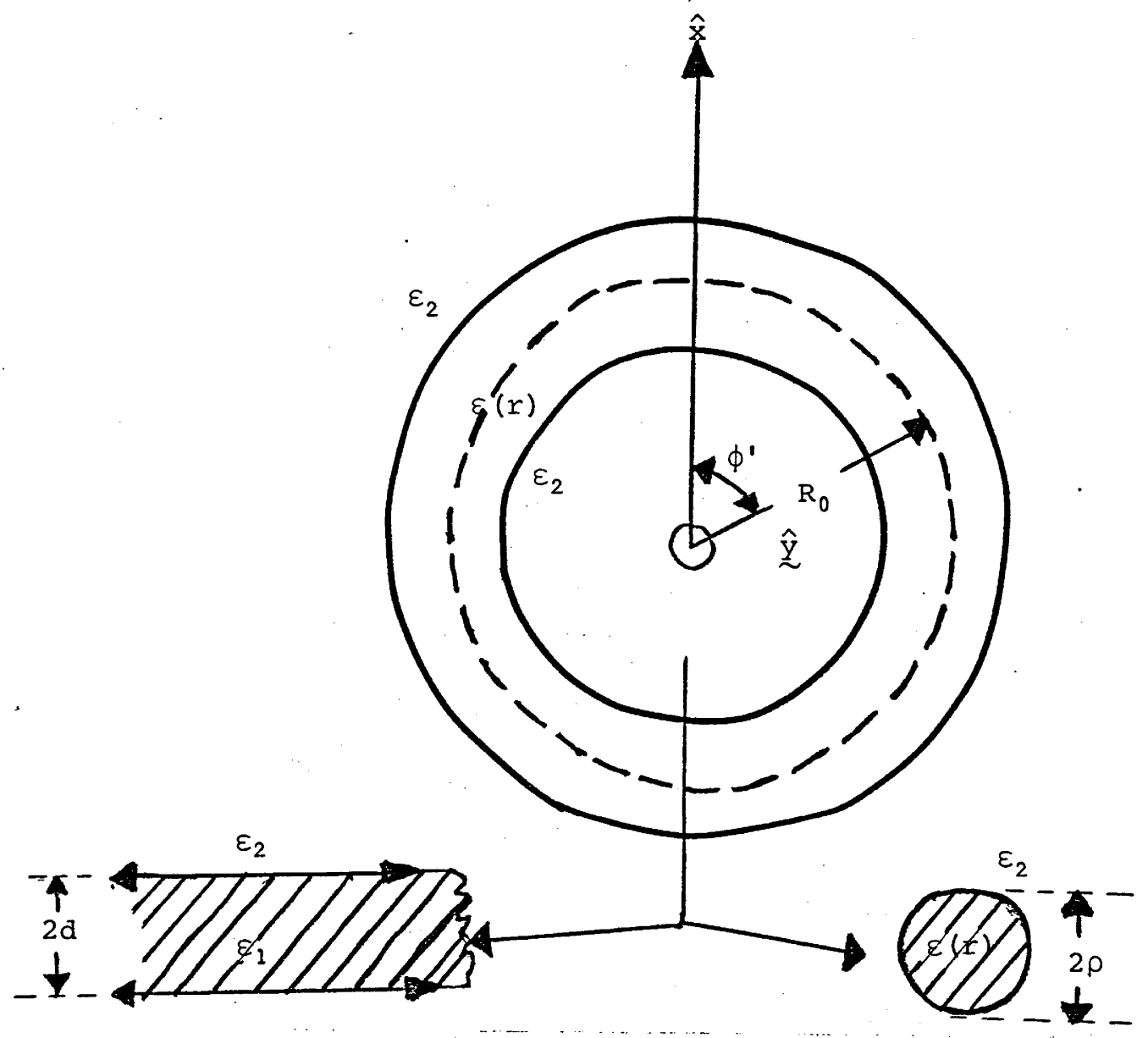

(a) Slab

(b) Fibre

Fig. 2: The complete planar bent structure for two and threedimensional waveguides. The cross sectional shapes of the waveguides are shown (a) and (b) respectively. 


\subsection{RADIATION FROM THE BENT \\ SYMMETRIC SLAB WAVEGUIDE}

In this section, we shall use the Surface Current Method, as elucidated in section 2 , to determine the modal power attenuation coefficients of the modes of the symmetric slab waveguide due to a bend of constant radius of curvature $R_{0}$. In particular, we shall consider the even TE modes. The result will then be compared with the result derived by the generalised. Fresnel's law for rays incident on a curved dielectric interface, first reported by snyder and Mitchell [19]. This comparison provides valuable insight into the approximations used in the Surface Current Method.

Using the philosophy of section 2, the fields in the annulus of thickness, $2 d$, with the constant radius of curvature, $R_{0}$, of the axis, as in fig. $2 a$, are the fields of the locally straight slab waveguide. Thus, considering the even TE modes of the symmetric slab waveguide [25], and using equation (2.2), the fields at the outer core-cladding surface ${ }^{\dagger}$ of the annulus in the local cartesian co-ordinate system $x^{\prime}, y^{\prime}, z^{\prime}$ relative to the waveguide axis (as in fig. 3) are

$$
\left.\begin{array}{l}
E_{y^{\prime}}=\cos U e^{-i m \phi^{\prime}} \\
H_{x^{\prime}}=-\left(\frac{\varepsilon_{2}}{\mu}\right)^{\frac{1}{2}} \frac{\beta}{k_{2}} \cos U e^{-i m \phi^{\prime}} \\
H_{z^{\prime}}=-\left(\frac{\varepsilon_{2}}{\mu}\right)^{\frac{1}{2}} \frac{i U}{k_{2} d} \sin U e^{-i m \phi^{\prime}},
\end{array}\right\}
$$

where

$$
m=\beta R_{0},
$$

where $\beta$ is the longitudinal propagation constant of the straight slab mode and

$$
\mathrm{U}=\rho\left(k_{1}^{2}-\beta^{2}\right)^{\frac{1}{2}}
$$

with $k_{1}$ and $k_{2}$ the wavenumbers of the core and cladding, respectively.

\footnotetext{
+ The outer surface, i.e. the circle with radius $\left(R_{0}+d\right)$, is the only surface needed in the Surface Current Integral since it encloses the entire two-dimensional volume together with the surface at infinity which does not contribute to the radiation field.
} 


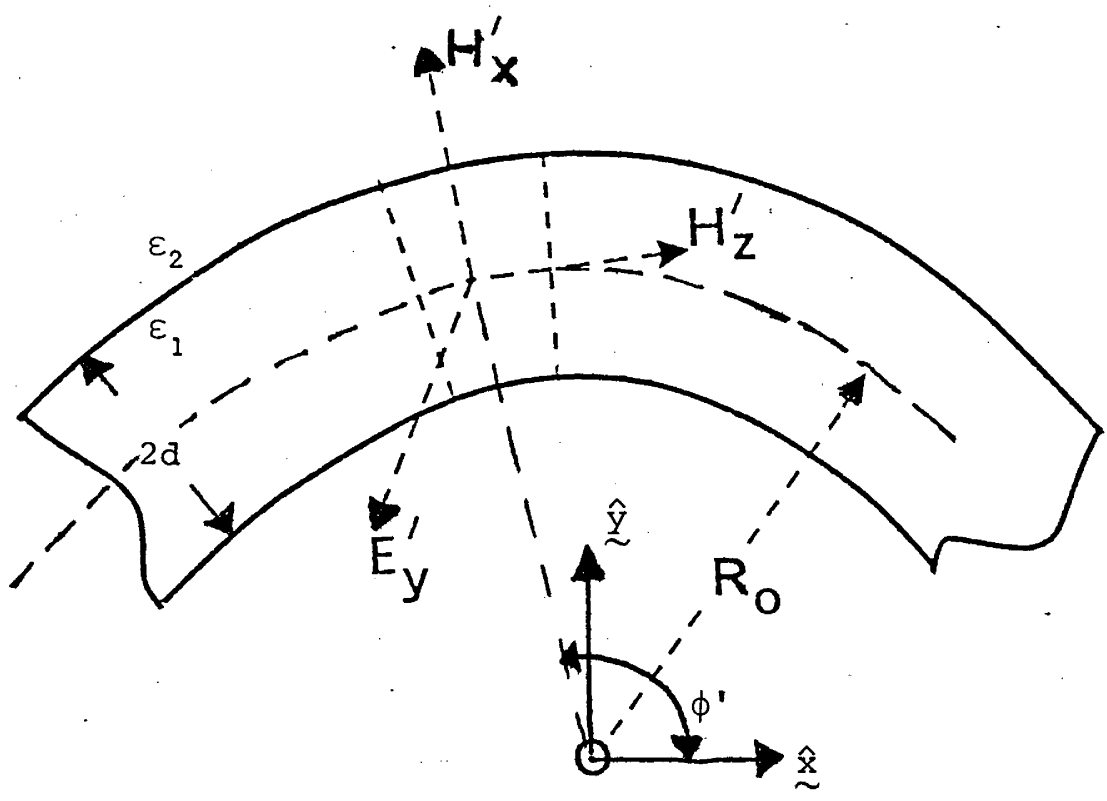

Fig. 3: Section of a bent planar (slab) waveguide. Each elemental segment of the core can be considered as a straight waveguide and its local fields and their directions can be related to the cartesian system relative to centre of curvature. 
The local unit vector normal to the interface is $\hat{x}^{\prime}$, so that the pseudo electric and magnetic surface currents, from equations $(5.2 .2 a, b)$, become

and

$$
\begin{aligned}
\left(\frac{\mu}{\varepsilon_{2}}\right)^{\frac{1}{2}} \underset{\mathrm{K}_{\mathrm{e}}}{\mathrm{K}} & =-\left(\frac{\mu}{\varepsilon_{2}}\right)^{\frac{1}{2}} \underset{\sim}{\hat{\mathrm{n}} \times \underset{\sim}{H}} \\
& =-\frac{i U}{\mathrm{k}_{2} \mathrm{~d}} \sin U \mathrm{e}^{-i m \phi^{\prime}} \hat{\underline{\mathrm{y}}}^{\prime}
\end{aligned}
$$

$$
\begin{aligned}
\cdot \underset{\sim}{\mathrm{K}} & =\hat{\sim} \stackrel{\sim}{x}^{\mathrm{E}} \\
& =\cos U \mathrm{e}^{-i m \phi^{\prime}} \hat{\underline{z}}^{\prime} .
\end{aligned}
$$

Transforming the local cartesian co-ordinate system to the global cartesian co-ordinate system with the origin at the centre of curvature, and using the formulae of the Surface Current Method, equation (5.2.8), we find the total power radiated, $\mathrm{P}^{\mathrm{R}}$, from the annulus is (see Appendix A)

$$
\mathrm{P}^{\mathrm{R}}=\left(\frac{\varepsilon_{2}}{\mu}\right)^{\frac{1}{2}} \frac{\mathrm{k}_{2} \pi^{2}\left(\mathrm{R}_{0}+\mathrm{d}\right)^{2} \mathrm{U}^{2}}{2 \mathrm{~V}^{2}}\left(\frac{\mathrm{W}}{\mathrm{k}_{2} \mathrm{~d}} J_{\mathrm{m}}(\mathrm{Y})+\mathrm{J}_{\mathrm{m}}^{\prime}(\mathrm{Y})\right)^{2},
$$

where the $J_{m}(Y)$ are Bessel functions of the first kind [36] with order $m=\beta R_{0}$ and argument

$$
Y=k_{0}\left(R_{0}+d\right)
$$

and the ' indicates differentiation with respect to $\mathrm{Y}$.

The power in the incident mode, with fields defined in equation (1) is $P_{0}$, where

$$
\mathrm{P}_{0}=\left(\frac{\varepsilon_{2}}{\mu}\right)^{\frac{1}{2}} \frac{\beta d(1+W)}{2 \mathrm{k}_{2} \mathrm{~W}}
$$

and the modal power attenuation coefficient of the incident even TE mode of the slab waveguide as it travels around the bent waveguide is

$$
\alpha=\frac{P^{R}}{2 \pi R_{0} \cdot P_{0}}
$$

which from equations (6) and (7) becomes 


$$
\alpha=\frac{\pi\left(R_{0}+d\right) k_{2}^{2} W U^{2}}{2 \beta d(I+W) V^{2}}\left(\frac{W}{k_{2} d} J_{m}(Y)+J_{m}^{\prime}(Y)\right)^{2} .
$$

Since the incident mode of the straight optical waveguide is a bound mode

$$
\beta>k_{2}
$$

and thus the order of the Bessel functions, $m=\beta R_{0}$, is greater than the argument, $k_{2}\left(R_{0}+d\right)$, and the near Debye asymptotic approximations for the Bessel functions can be used [36], in equation (9). By the application of these asymptotic forms (Appendix A, equation (A.23)) in equation (9), together with a Taylor series expansion of the exponent to first order in $d / R_{0}$, we find, equation (9), for the power attenuation coefficient of the mode becomes

$$
\alpha=\frac{W^{2} U^{2}}{\beta d^{2}(1+W) V^{2}} \exp \{2 W-2 \pi(\beta, w)\}
$$

where

$$
\pi(\beta, w)=\frac{R_{0}}{d}\left\{w-\beta d \tanh ^{-1}\left(\frac{w}{\beta d}\right)\right\},
$$

where

$$
W=\rho\left(\beta^{2}-k_{2}^{2}\right)^{\frac{1}{2}} \text {. }
$$

To obtain equation (10), we have used a power series expansion of the exponent of the asymptotic approximation to the Bessel function, in powers of $d / R_{0} \ll 1$, and retained only the first order expansion in $d / R_{0}$. For this to be valid we require the remaining terms to be much less than 1 , so that considering the second order expansion in $\left(d / R_{0}\right)$, we require

$$
\frac{R_{0}}{d}>\frac{(\beta d)^{2}}{2 W} .
$$

For weakly guiding waveguides $W / B d<1$, so that using a power series expansion of $\tanh ^{-1}(x)$ [37] for $x<1$ in equation (11), for $\pi\left(m, Y_{0}\right)$, we find that equation (10) can be re-written as

$$
\alpha=\frac{W^{2} U^{2}}{\beta d^{2}(1+W) V^{2}} \exp \left\{2 W-\frac{2}{3} \frac{W^{3}}{(\beta d)^{2}}\left(\frac{R_{0}}{d}\right)\right\}
$$

which is valid for 


$$
\frac{5 V^{4}}{2 w^{5} \theta_{C}^{4}} \gg \frac{R_{0}}{d} \gg \frac{(\beta d)^{2}}{2 W} \sim \frac{V^{2}}{2 w \theta^{2}} .
$$

In this form, equation (14), the power attenuation coefficient of the even TE modes of the slab waveguide is seen to agree with the well known result [25].

From our heuristic discussion of the bending loss of a dielectric optical fibre, we isolated those parameters which significantly affect the susceptibility of a mode to bending losses, i.e. $V, \theta_{c^{\prime}}$ w. It would be profitable to write equation (14) in such a notation. For weakly guiding waveguides we can rewrite equation (14) as (Appendix A, equation (A.37))

$$
\alpha=\frac{U^{2} W^{2}}{B d^{2}(1+W) v^{2}} \exp \left\{2 W-\frac{2}{3} \frac{W^{2}}{v^{2}} \theta_{c}^{2}\left(\frac{R_{0}}{d}\right)\right\}
$$

which is valid for the range of aspect ratios $\left(R_{0} / d\right)$ defined in equation (15). In this form, we can see that these parameters critically affect the magnitude of the radiation from a mode due to a bend of constant radius. For modes far from cutoff, $W / V \sim 1$, so that as $V$ increases $\mathrm{W}^{3} / \mathrm{V}^{2} \rightarrow \mathrm{V}$ and thus the radiation loss of the mode decreases rapidly. Similarly if we keep $V$ constant, thereby fixing $w$, and vary the acceptance angle of the slab waveguide, we see that the radiation increases dramatically as $\theta_{C}$ is decreased. This is the main reason that waveguides are not manufactured with arbitrarily small dielectric differences between the core and cladding, ${ }^{\dagger}$ since their susceptibility of the slab modes to bending losses increases dramatically.

In Appendix B, we have used the generalised Fresnel's law for rays incident at a curved core-cladding interface [19] to determine the attenuation coefficients of the rays of a slab waveguide. This analysis closely follows the original derivation by snyder and Mitchell [19]. As the modes of the slab waveguide are made up of a family of rays with the same angle $\theta_{z}$ to the waveguide axis, it would appear that by studying the radiative loss from one of the rays of the family that constitute

\footnotetext{
$\dagger$ The arbitrarily small dielectric difference between the core and cladding would appear to be very attractive since the pulse dispersion of the waveguide could be substantially reduced.
} 
the mode, we could re-derive the attenuation coefficient of the modes of the slab waveguide.

In order to clarify the process, we consider a finite segment of a bend ${ }^{\dagger}$ into which the rays incident from the straight slab enter as in fig. 4. We consider the two rays $A$ and $B$ of the mode family, each corresponding to power in the mode entering at a different distance from the centre of curvature of the bend. In the bent section, the two rays which were parallel in the straight slab are incident on the outer corecladding interface at different angles, viz.

$$
\theta_{i}^{A}=\theta_{z}
$$

and

$$
\theta_{i}^{B}=\theta_{z}+\varepsilon,
$$

where for $d / R_{0}<<1$ (Appendix $B$ )

$$
\varepsilon=\frac{d}{R_{0}} \cot \theta_{z}
$$

The generalised Fresnel's law transmission coefficient, T, [19] for a ray incident on a curved dielectric interface at an angle $\theta_{i}$ to the tangent to the interface, is

$$
T=\frac{4\left(\sin ^{2} \theta_{c}-\sin ^{2} \theta_{i}\right)^{\frac{1}{2}}}{\sin \theta_{i}} \exp \left\{-\frac{2 k_{1} R_{0}\left\{\sin ^{2} \theta_{c}-\sin ^{2} \theta_{c}\right\}^{\frac{3}{2}}}{3 \cos ^{2} \theta_{i}}\right\}
$$

for rays with

$$
\frac{\theta_{i}}{\theta_{c}} \sim 1
$$

where

$$
\theta_{C}=\sin ^{-1}\left(1-\frac{\varepsilon_{2}}{\varepsilon_{1}}\right)^{\frac{1}{2}} \ll 1 \text {. }
$$

Thus, from equations (17) - (20), it can be seen that the two rays $A$ and $B$ suffer different amounts of loss from the curved interface. If we assume that the rays each suffer the same number of reflections from this outer-cladding interface, then the attenuation of the ray per unit

t we ignore the electromagnetic phenomena of the scattering of the rays due to the change in the curvature. 


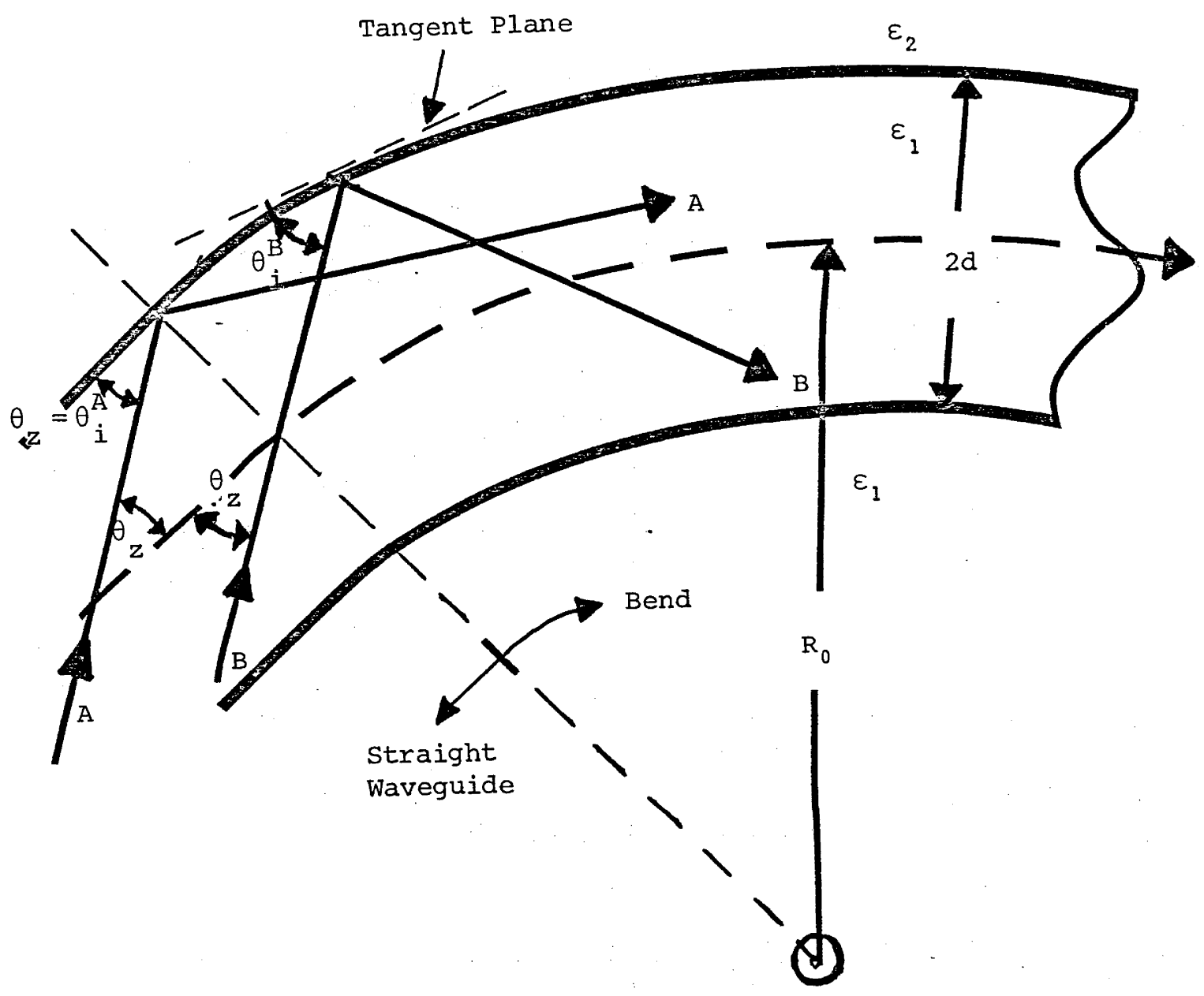

Fig. 4: The two rays $A$ and $B$ of the family of rays of the slab waveguide mode, each incident at the outer core-cladding interface at different angles $\theta_{i}^{A}=\theta_{z}$ and $\theta_{i}^{B} \neq \theta_{z}$, where $\theta_{z}$ is the angle of incidence in the straight waveguide. 
length of the bend, $\alpha$, is

$$
\alpha=\mathrm{N} T
$$

where $\mathrm{N}$ is the number of reflection from this interface per unit length of waveguide. Including the Goos-Hänchen shift [38] for rays incident at angles less than $\theta_{C^{\prime}}$, we have (Appendix B, equation B.6))

$$
\begin{gathered}
\alpha=\frac{k_{2}\left(\sin ^{2} \theta_{c}-\sin ^{2} \theta_{i}\right)}{\cos ^{2} \theta_{i}\left[1+k_{2} d\left\{\sin ^{2} \theta_{c}-\sin ^{2} \theta_{i}\right\}^{\frac{1}{2}}\right]} \\
\times \exp \left\{-\frac{2}{3} \frac{k_{1} R_{0}}{\cos ^{2} \theta_{i}}\left\{\sin ^{2} \theta_{c}-\sin ^{2} \theta_{i}\right\}^{\frac{3}{2}}\right\},
\end{gathered}
$$

where $R_{0}$ is the radius of curvature in the plane of incidence for a ray incident at angle $\theta_{i}$ to the interface where $\theta_{i} / \theta_{C} \sim 1$.

Thus, using equation (22) for rays $A$ and $B$ with angles of incidence $\theta_{i}^{A}, \theta_{i}^{B}$ respectively, the attenuation coefficients for the two rays using a power series expansion in $d / R_{0}$ in the exponent of equation (22) for the angle $\theta_{i}^{B}$ become

$$
\alpha_{B}=\frac{w^{2}}{k_{2} d^{2}(I+W)} \exp \left\{2 W-\frac{2 W^{3}}{3(\beta d)^{2}}\left(\frac{R_{0}}{d}\right)\right\}
$$

and

$$
\frac{\alpha_{B}}{\alpha_{A}}=e^{2 W} \text {, }
$$

where we have used the usual transformation between mode parameters and rays [39]

and

$$
\begin{aligned}
& \left.\begin{array}{l}
U=k_{1} d \sin \theta_{z} \\
V=k_{1} d \sin \theta_{C}
\end{array}\right\} \\
& W=\left(V^{2}-U^{2}\right)^{\frac{1}{2}}=k_{1} d\left(\sin ^{2} \theta_{C}-\sin ^{2} \theta_{i}\right)^{\frac{1}{2}} \\
& B=k_{1} \cos \theta_{z} .
\end{aligned}
$$

Employing equations (25) in equation (21), this formula, equation (23) is valid for $\mathrm{U} / \mathrm{V} \sim \mathrm{l}$. 
From a comparison of equation (23) with equation (14) derived by the modal analysis of the Surface Current Method, for $U / V \sim 1$, we find

$$
\alpha_{B}=\alpha_{\text {mode }}
$$

where $\alpha_{\text {mode }}$ is the Surface Current Method result for $U / V \sim I$, i.e. equation (14).

From equation (26) then, we can say that the ray optical analysis of the bending loss for slab waveguides agrees with the modal result calculated by the Surface Current Method if we choose the ray with angle, $\theta_{z}$, to the axis, in the curved waveguide to characterise the family of rays that make up the mode. From equation (24) we can see that the choice of ray that characterises the family is critical in determining the radiation loss.

From the above analysis, we can also observe that this modified geometric optics argument does not distinguish between symmetric and asymmetric dielectric waveguides, ${ }^{\dagger}$ as the loss depends only on the angle of incidence of the ray.

Now that we have displayed the physical significance of the phase matching procedure in the bent optical waveguides with reference to the geometric optics analogue, we shall now proceed to the principally important situation of bends in weakly guiding dielectric optical waveguides.

\subsection{RADIATION FROM THE BENT OPTICAL FIBRE}

In the last section, the Surface Current Method was used to calculate the radiative loss and its associated power attenuation coefficient for the even TE modes of the symmetric slab waveguide due to a bend of a constant radius of curvature. In this section, we shall use the surface Current Method to determine the modal power attenuation

\footnotetext{
+ The attenuation coefficient of rays in asymmetric slab waveguides does contain some asymmetry dependence due to the effect of the GoosHänchen shifts at the two dielectric interfaces, but as Chang and Kuester [15] show, this is not the dominant asymmetric dependence.
} 
coefficients of the $\mathrm{EH}$ and $\mathrm{HE}$ modes of the dielectric optical fibre of circular cross section, employing the general modal formalism of Chapter 2. Quantitative results are plotted for the particular case of weakly guiding step-index fibres. Finally, in this section, quantitative results by this analysis are compared with the experimental results available, and a discussion of the effects that have been neglected, is presented.

In the ensuing analysis, we use the azimuthal travelling wave modal formulation of Chapter 2 in preference to the azimuthal standing wave modes. We choose this form for two reasons:

(1) The relative simplicity of the evaluation of the surface Current Integrals for the azimuthal travelling wave modes, in comparison to the azimuthal standing wave modes.

(2) The direct geometric optics interpretation of the propagation of the azimuthal travelling wave modes as rays travelling down the fibre in a broken helical path clockwise and anticlockwise in the azimuthal direction depending on the sign of the azimuthal wave number $l$. Such a physical picture allows a ready interpretation of the analysis via the generalised Fresnel's law for rays incident on a curved dielectric interface.

As for the slab waveguide, we analyse the radiative loss from a complete torus, with the radius of curvature of the fibre axis, $R_{0}$, as in fig. 2 .

The time averaged Poynting vector, $s^{R}$, of the radiation from a positive travelling azimuthal wave mode (i.e. $\ell>0$ ) by equation (5.2.12), is

$$
S^{R}=\left(\frac{\varepsilon_{2}}{\mu}\right)^{\frac{1}{2}} \frac{k_{2}^{2}}{16(\pi R)^{2}}|\underset{\sim}{\Gamma} \cdot(\underset{\sim}{\hat{x}} \pm i(\underset{\sim}{\hat{y}} \cos \theta-\underset{\sim}{\hat{z}} \sin \theta))|^{2},
$$

where the + and - signs refer to the $\mathrm{HE}_{\ell}$ and $\mathrm{EH}_{\ell}$ modes respectively,

$$
\underset{\sim}{\sim}=\int_{S^{\prime}}(\stackrel{n}{\sim} \times \underset{\sim}{E}) \exp \left\{i k_{2} \underset{\sim}{\stackrel{\hat{R}}{\sim}} \cdot \mathbb{R}^{\prime}\right\} d A,
$$


where for the Surface Current calculations the surface $S^{\prime}$ is chosen to be the core-cladding interface of the curved optical fibre.

The tangential components of the electric field at the corecladding interface, $i . e . \mathrm{E}_{z}$, and $\mathrm{E}_{X}$ (where $X$ is now the azimuthal variation) are, from equations (2.2.43) - (2.2.45)

and

$$
\left.\begin{array}{l}
E_{\chi^{\prime}}=A_{\ell \mp I} e^{-i m \phi^{\prime}-i \ell \chi} \\
E_{z^{\prime}}=-A_{\ell \mp 1} B_{\ell \mp 1} e^{-i m \phi^{\prime}-i \ell \chi},
\end{array}\right\}
$$

where $r^{\prime}, \chi_{,} z^{\prime}$ are the local cylindrical co-ordinates of the fibre relative to the waveguide axis, as in fig. 5. We have used the phase matching procedure along the fibre axis so that we can write

$$
\beta z \rightarrow \beta R_{0} \phi^{\prime}=m \phi^{\prime},
$$

i.e.

$$
\beta R_{0}=m \text {, }
$$

where $\phi^{\prime}$ is the angular variation around the axis of the bent fibre as illustrated in fig. $5 \mathrm{a} . \mathrm{B}_{\ell \mp l}$ is the magnitude of the longitudinal field at the core-cladding interface, which from equations (2.2.43)-(2.2.45) becomes

$$
B_{\ell \mp 1}=\frac{i W}{k_{2} \rho} \frac{H_{\ell_{\ell \mp 1}^{(I)}(i W)}^{(I)}(i W)}{H_{\ell}}
$$

for all dielectric waveguides of circular cross section. $A_{\ell \mp I}$ is the power normalisation coefficient of the mode defined in equation (2.2.53) chosen so that unit power propagates in the mode. The subscripts $l+1$ and $\ell-1$ refer to the $\mathrm{EH}_{\ell}$ mode and the $\mathrm{HE}_{\ell}$ mode of the weakly guiding waveguide, respectively, as usual.

The local unit vector normal to the core-cladding interface is the unit radial vector of the local cylindrical co-ordinate system, so that

$$
\underset{\sim}{\tilde{n}} \times \underset{\sim}{E}=-E_{z} \hat{\chi}+E_{X} \underset{\sim}{\hat{z}^{\prime}}
$$




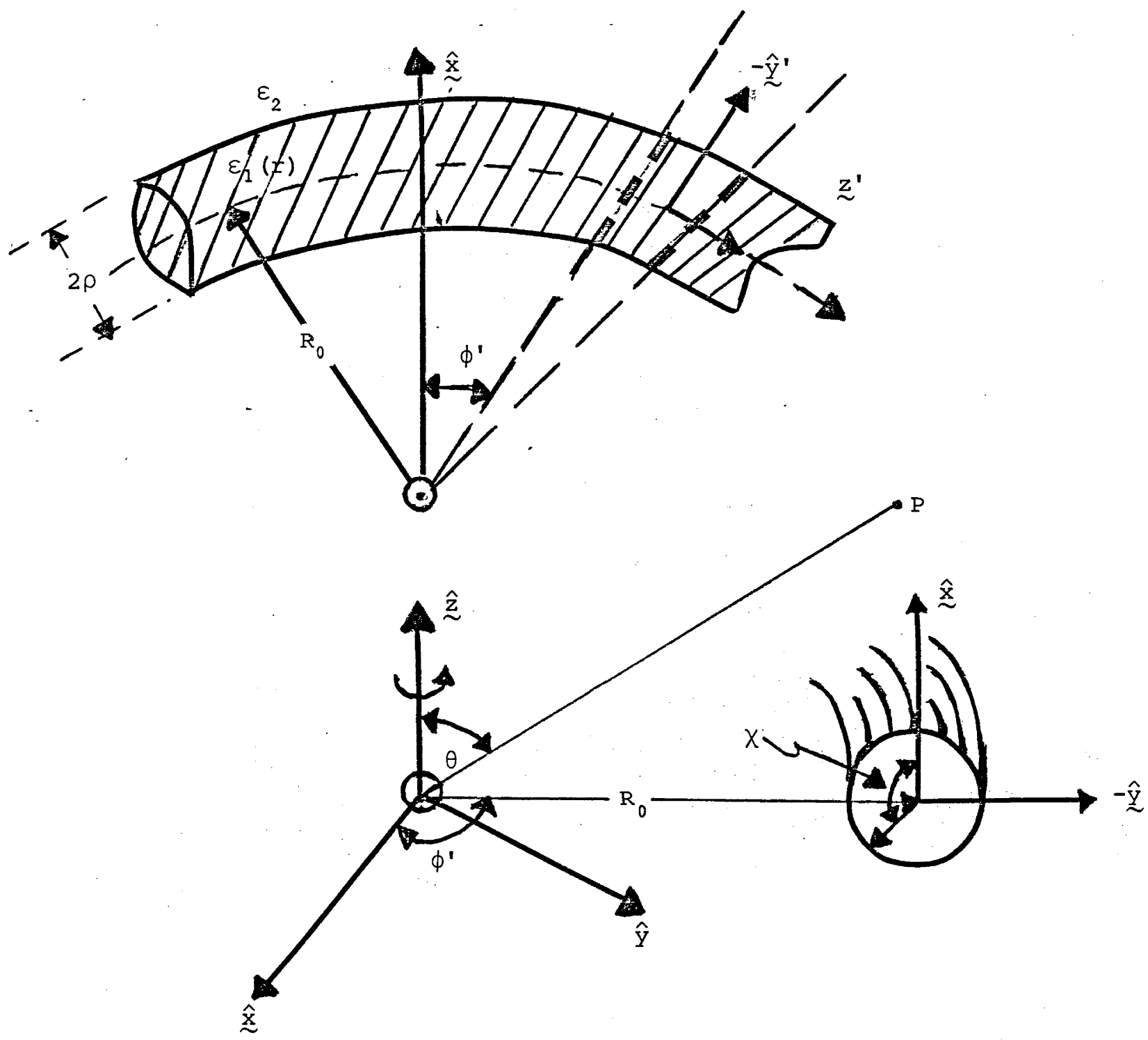

Fig. 5: The co-ordinate systems of the toroidal structure. $\underset{\sim}{\hat{x}^{\prime}}, \hat{\mathbf{y}}^{\prime}, \hat{\sim}^{\prime}$ are the local cartesian co-ordinates relative to the waveguide axis and $\underset{\mathbf{x}}{\hat{\mathbf{y}}}, \hat{z}$ are the global cartesian co-ordinates relative to the centre of curvature 0 . 
Using this in equation (2), the asymptotic evaluation of the integral provides the result for the time averaged Poynting vector, $s^{R}$, of the radiation from equation (1). Due to the symmetry of the radiation from a toroid, the radiant energy flux need only be evaluated at one point around the axis of the toroid, and then the total power radiated, $\mathrm{P}^{\mathrm{R}}$, is

$$
P^{R}=2 \pi R^{2} \int_{0}^{\pi} S_{R} \sin \theta d \theta
$$

where $\theta$ is as defined in fig. $5 b$.

This integral can only be evaluated asymptotically for the bent fibre, and from Laplace's Method [40] for the asymptotic evaluation of equation (7) we find that the maximum contribution to the radiation occurs for the angle $\theta_{0}$, where (Appendix C)

$$
\theta_{0}=\frac{\pi}{2}-\psi
$$

and

$$
\left.\begin{array}{ll}
\psi=0 & \text { for } \ell \sim 1 \\
\psi=\frac{\ell k_{2} \rho^{2}}{W^{2} R_{0}} & \text { for } \ell>1
\end{array}\right\} \text {. }
$$

Since $\rho / R_{0}<1$, the radiation from the bent optical waveguide is confined to near the plane of bending, i.e. $\theta=\pi / 2$ from fig. $5 b$. From this asymptotic evaluation of the total power radiated from the toroid we find (ignoring all terms of order $\rho / R_{0}$ or smaller, except in the exponent) (Appendix $C$ )

$$
\mathrm{P}^{\mathrm{R}}=\pi^{2} \mathrm{R}_{0}\left(\frac{\varepsilon_{2}}{\mu}\right)^{\frac{1}{2}}\left(\frac{\pi \rho}{W R_{0}}\right)^{\frac{1}{2}} \frac{\rho}{W}\left(\frac{\mathrm{A}_{\ell \mp 1}}{\mathrm{~K}_{\ell \mp I}(W)}\right)^{2} \exp \left\{x_{\ell}\left(\beta, R_{0}\right)\right\},
$$

where $\mathrm{K}_{\ell \mp 1}(W)$ is the modified Bessel function of the second kind [36], and the subscripts $\ell+1$ and $\ell-1$ refer to the $\mathrm{EH}_{\ell}$ and $\mathrm{HE}_{\ell}$ modes respectively, where

$$
X_{\ell}\left(\beta, R_{0}\right)=2\left(\frac{R_{0}}{\rho}\right)\left\{w-\beta \rho \tanh ^{-1}\left(\frac{W}{\beta \rho}\right)\right\}+\xi_{\ell^{\prime}}
$$

where 


$$
\left.\begin{array}{rlr}
\xi_{\ell}=0 & \text { for } l \sim 1 \\
=\frac{\ell^{2} k_{2}^{2} \rho^{3}}{R_{0} W^{3}} \ll 1: & \text { for } l>1
\end{array}\right\}
$$

and this result, equation (10), is valid for all radii of curvature satisfying

$$
\frac{R_{0}}{\rho}>\frac{(\beta \rho)^{2}}{2 W}
$$

This criteria for the validity of equation (10) is identical to the criteria for the slab waveguide, equation (3.13).

The Laplace Method for evaluating the integral of equation (7) is based upon the approximation of the function, about its point of maximum contribution, by a Gaussian [40]. For the asymptotic evaluation to be valid, we require that the function is strongly concentrated about this point. Thus, a measure of the validity is given by the half-width of the Gaussian function approximating the integrand-if the half-width of the Gaussian is small, then the approximation is very good. For the evaluation of equation (7) the angular half-width $\Delta \theta$, about the point of maximum contribution, is (Appendix C, equation (C.40))

$$
\Delta \theta \sim\left(\frac{\rho}{2 R_{0} W}\right)^{\frac{1}{2}} \ll 1
$$

since $\rho / R_{0}<1$. Thus, the radiated power is strongly confined about the direction of maximum radiation, $\theta_{0}$.

Since the incident modal field was normalised to unit guided power, the modal attenuation coefficient $\alpha$ for the mode propagating around the toroid of axial circumference, $2 \pi R_{0}$, is

$$
\alpha=\frac{\mathrm{P}^{\mathrm{R}}}{2 \pi \mathrm{R}_{0}}
$$

and therefore, using equation (10), becomes

$$
\alpha=\frac{\pi}{2}\left(\frac{\varepsilon_{2}}{\mu}\right)^{\frac{1}{2}}\left(\frac{\pi \rho}{W R_{0}}\right)^{\frac{1}{2}} \frac{\rho}{W}\left(\frac{A_{l \mp 1}}{K_{l \mp 1}(W)}\right)^{2} \exp \left\{x_{\ell}\left(\beta, R_{0}\right)\right\} .
$$


For the step-index weakly guiding dielectric optical fibre, the power normalisation coefficient is given in equation (2.3.16), and thus the power attenuation coefficient for the fibre mode is

$$
\alpha=\frac{1}{2 \rho}\left(\frac{\pi \rho}{W R_{0}}\right)^{\frac{1}{2}} \frac{U^{2}}{W V^{2}}\left(K_{\ell \mp 2}(W) \cdot K_{\ell}(W)\right)^{-1} \exp \left\{x_{\ell}\left(B, R_{0}\right)\right\}
$$

which is valid for $R_{0} / \rho>>(\beta \rho)^{2} / 2 w$.

For weakly guiding dielectric optical fibres $W / B \rho \ll 1$ and we may use the approximations of the exponential as detailed in section 3 , so that the power attenuation coefficient for weakly guiding dielectric optical fibres may be written as

$$
\alpha=\frac{\pi}{2}\left(\frac{\varepsilon_{2}}{\mu}\right)^{\frac{1}{2}}\left(\frac{\pi \rho}{W R_{0}}\right)^{\frac{l}{2}} \frac{\rho}{W}\left(\frac{A_{l \mp I}}{K_{l \mp 1}(W)}\right)^{2} \exp \left\{-\frac{2}{3} \frac{W^{3}}{V^{2}} \theta_{C}^{2}\left(\frac{R_{0}}{\rho}\right)+\xi_{\ell}\right\},
$$

where $\xi_{\ell}$ is as defined in equation (12), and this formula for $\alpha$ is valid for $R_{0} / \rho$ in the range

$$
\frac{5 v^{4}}{2 w^{5} \theta_{c}^{4}}>\frac{R_{0}}{\rho}>\frac{v^{2}}{2 w \theta_{c}^{2}}
$$

by a similar argument to that of section 3 .

The lower limit on the applicability of equation (18), i.e.

$$
\frac{R_{0}}{\rho} \gg \frac{V^{2}}{2 w \theta^{2}}
$$

is a more stringent requirement than that reported by Chang and Kuester, equation (28) of ref. 16, since from equation (20) we find,

$$
\frac{2}{3} \frac{w^{3}}{v^{2}} \cdot \theta_{c}^{2}\left(\frac{R_{0}}{\rho}\right) \gg \frac{W^{2}}{3}
$$

since for multimode waveguides $W>1$ for modes far from cutoff.

From equations (16) and (18), the only difference between the bending losses of the step-index and graded-index optical waveguides is in the power normalisation coefficient $A_{\ell \mp 1}$ and the waveguide and modal 
parameters $\rho$ and $W$ for waveguides with identical $V$ values, where for graded index waveguides

$$
\begin{aligned}
V & =k_{1} \rho\left(1-\frac{\varepsilon_{2}}{\varepsilon_{1}}\right)^{\frac{1}{2}} \\
& =k_{1} \rho \theta_{C},
\end{aligned}
$$

where $\varepsilon_{1}$ is the dielectric permittivity in the core of the fibre on the fibre axis for the graded-index fibre, and $\varepsilon_{2}$ is the dielectric permittivity of the cladding. $k_{1}$ is the wavenumber of the light evaluated at the axis of the fibre.

Thus, the Surface Current Method provides a method of calculation that does not discriminate between the types of dielectric profile of the core of the optical fibre.

Equation (17) for the step-index dielectric optical fibre is in exact agreement with previously reported results $[7-12,15]$, excluding the additional exponential factor $\xi_{l}$ defined in equation (12). However, for $\rho / R_{0} \ll 1, \xi_{\ell} \ll 1$, and the results agree to a high degree of accuracy. Equation (17) is different from that reported by snyder, White and Mitchell [11] due to the omission of a factor of $e^{2 W}$ in ref. 11, and due to the use of the far from cutoff approximation of $w>1$, valid for multimode fibres only. An additional error in ref. 11 in the additional exponential term, $\xi_{l^{\prime}}$ of a factor of 2 , is due to a mathematical error on the part of the author of this thesis. However, since $\xi_{\ell} \ll 1$, this error does not affect the calculations presented in ref. 11 .

Let us now consider the form of this power attenuation coefficient and discuss the relevance of this result for the modal power attenuation coefficient of the azimuthal travelling wave modes to the physically realistic azimuthal standing wave modes.

In Appendix D, we demonstrate that the total power radiated from the azimuthal travelling wave mode can be directly used for the power attenuation coefficient for the azimuthal standing wave mode which is formed by addition of the two azimuthal travelling wave modes with 
the same magnitude of the azimuthal mode number, $l$, but opposite signs, and the same polarisation, i.e. HE or EH modes. in Appendix D, the positive and negative travelling azimuthal modes are shown to radiate equally, but their radiation patterns are, in general, mirror images of one another in the plane of the toroid.

This can be understood from a consideration of the ray optics analogue employing the generalised Fresnel's reflection coefficient. The azimuthal travelling wave mode with phase variation around the axis of $\exp \{-i \ell \phi\}$, where $\ell>0$, corresponds to a family of skew rays [39] travelling in the positive $\phi$ direction, as in fig. 6 , in the cross section of the fibre. Similarly, the mode with $\ell<0$ corresponds to the family of skew rays travelling in the opposite direction around the fibre cross section. As the magnitude of the loss due to curvature from the generalised Fresnel's law, equation (3.20), is strongly dependent on the radius of curvature of the curved surface in the plane of incidence of the ray, the tunnelling radiation of a ray in the bent fibre will be predominantly in the plane of the bend, where the radius of curvature is smallest. However, for the family of skew rays for which the plane of incidence of the ray, in which the tunnelling radiation propagates, is not coincident with the plane of the bend, the radiation due to the bend will be asymmetric relative to the plane of the torus, and the positive and negative travelling rays will radiate as shown in fig. $6 b$. Thus the azimuthal travelling wave modes would be expected to have asymmetric radiation patterns relative to the plane of the bend, as is found from the analysis of Appendix $C$.

Since the two families of positive and negative travelling skew rays each experience identical curvature of the core-cladding interface in the planar bend in the fibre, the total radiated power would be expected to be the same for the two rays and similarly for the two azimuthal travelling wave modes. This is mathematically demonstrated in Appendix D. We will continue to discuss the bending loss phenomena for the azimuthal travelling wave formulation, since from the above discussion the geometric optics equivalent is more obvious.

The additional radiation loss of the skew modes expressed by the additional term in the exponent, $\xi_{\ell}$, defined in equation $(12)$, is 

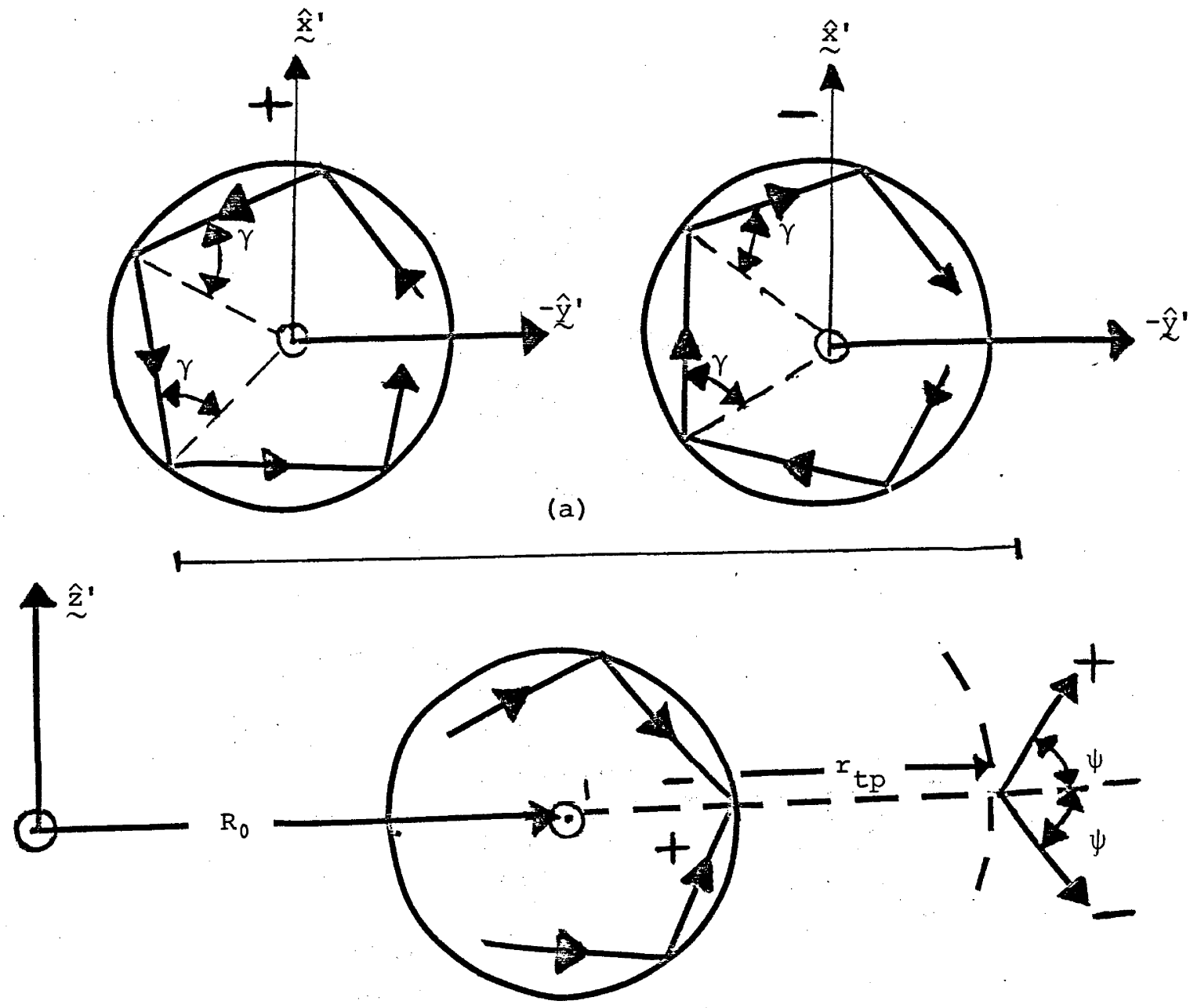

(b)

Fig. 6: The two azimuthal travelling wave modes, denoted by \pm signs, and their radiation loss due to a planar bend in the axis. 
due to the rays of the mode experiencing an additional curvature of the core-cladding interface in their plane of incidence due to the inherent curvature of the core-cladding interface in the fibre cross section. Although this additional term does have this valid interpretation, the accuracy of this correction is doubtful, since from Chang and Kuester's analysis [15], we have not included higher order corrections, in $\rho / R_{0}$, to the phase constant of the mode in the bend.

In fig. 7, we have plotted the attenuation coefficient $\alpha$ $(\mathrm{dB} /$ metre) of equation (17) against the acceptance angle of the waveguide for varying curvature radii for a fixed wavelength of the excitation field in a $V=2.2$ step-index waveguide. As can be seen from the rapid variation in the radiation loss with $\theta_{c^{\prime}}$, the modal power attenuation coefficient for the $\mathrm{HE}_{11}$ mode is highly sensitive to the acceptance angle of the fibre and the radius of curvature of the bend, $\mathrm{R}_{0}$.

In figs. 8a-d, we have plotted a similar result to fig. 7 , for a range of waveguides of $\mathrm{v}=1.2$ up to 2.4 , where we fixed the radius of the optical fibre, viz. $\rho=2 \mu$, and plot $\alpha$ (dB/metre) as a function of $\theta_{c}$ for a range of bending radii. The discrepancy of the range over $\theta_{C}$ plotted for the different curvature radii is due to the criteria for applicability of equation (16), viz. equation (13). From these plots, it can be seen that the radiation loss of a monomode fibre with an acceptance angle $\theta_{C} \sim 0.1 \mathrm{rad}$. is insignificant even for a radius of curvature of $1 \mathrm{~cm}$.

In the introduction, we mentioned that the formulae for the two-dimensional slab waveguide modal attenuation coefficients were often used to extrapolate to three-dimensional waveguides. We are now in a position to evaluate the validity of this extrapolation. From equation (3.14) and equation (17) using the far Debye asymptotic expression for the modified Bessel function of the second kind [36], we find, for modes far from cutoff,

$$
\frac{\alpha_{\text {slab }}}{\alpha_{\text {fibre }}} \sim \theta_{\mathrm{C}}\left(\frac{R_{0}}{\rho}\right)^{\frac{1}{2}}>1
$$




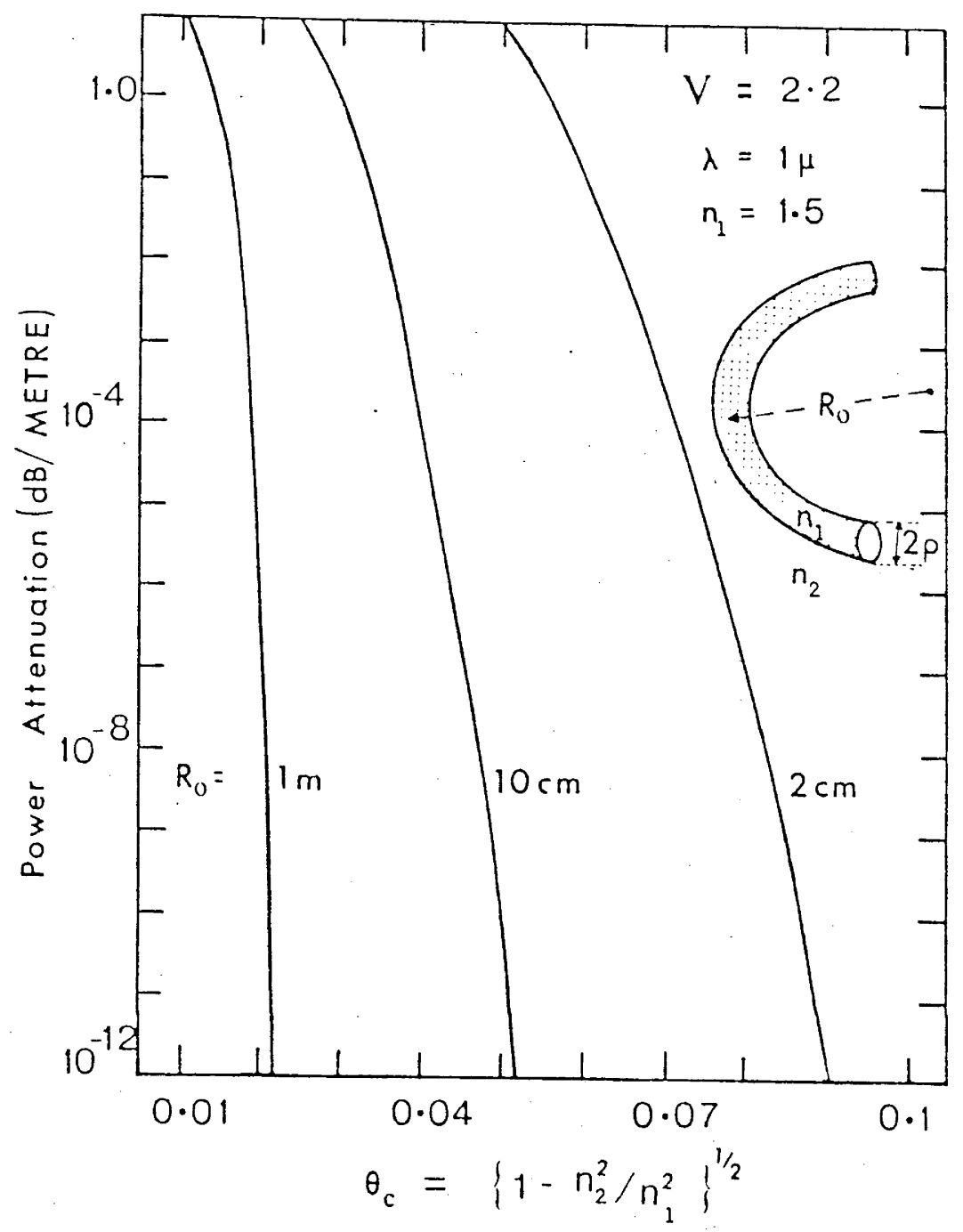

Fig. 7: The power attenuation (dB/metre) vs. the complement of the critical angle, $\theta_{C}=\left(1-\varepsilon_{2} / \varepsilon_{1}\right)^{\frac{1}{2}}$.

The power attenuation $P_{d B}=10 \log _{10}\{P(z) / P(0)\}=4.34 \alpha$ is plotted against $\theta_{C}$ for various values of the bending radius of the fibre $R_{0}$, where $\alpha$ is given by eq. (17). For $V=2.2, U=1.527$, $\mathrm{W}=1.584$ and $\alpha=\left\{5.1 /\left(\mathrm{R}_{0} \rho\right)^{\frac{1}{2}} \exp \left\{-5.5 \theta_{C}^{2} \mathrm{R}_{0} / \rho\right\}\right.$. 


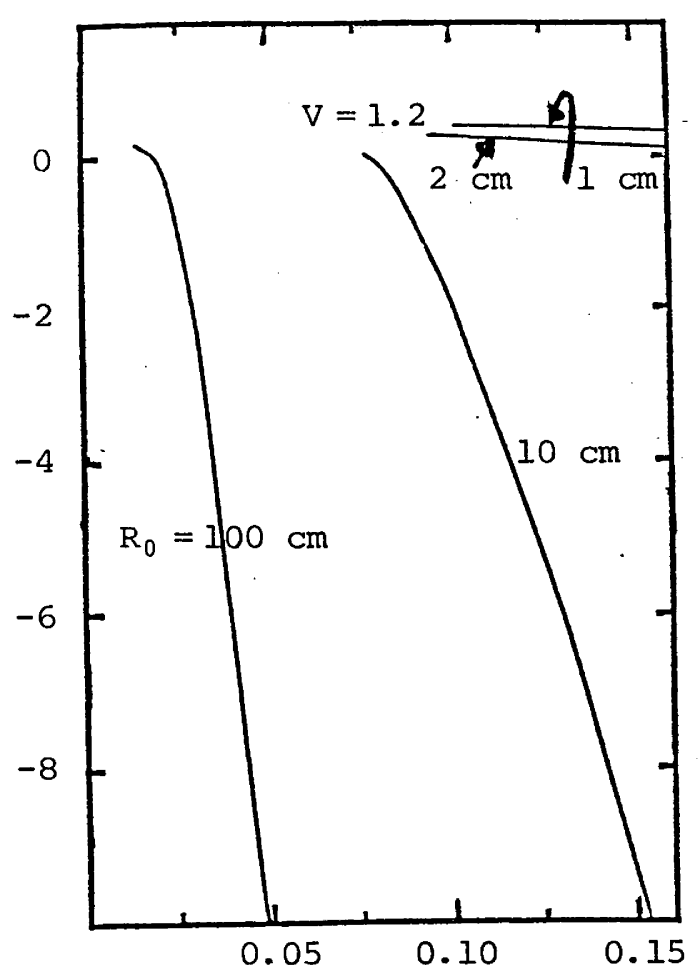

(a)

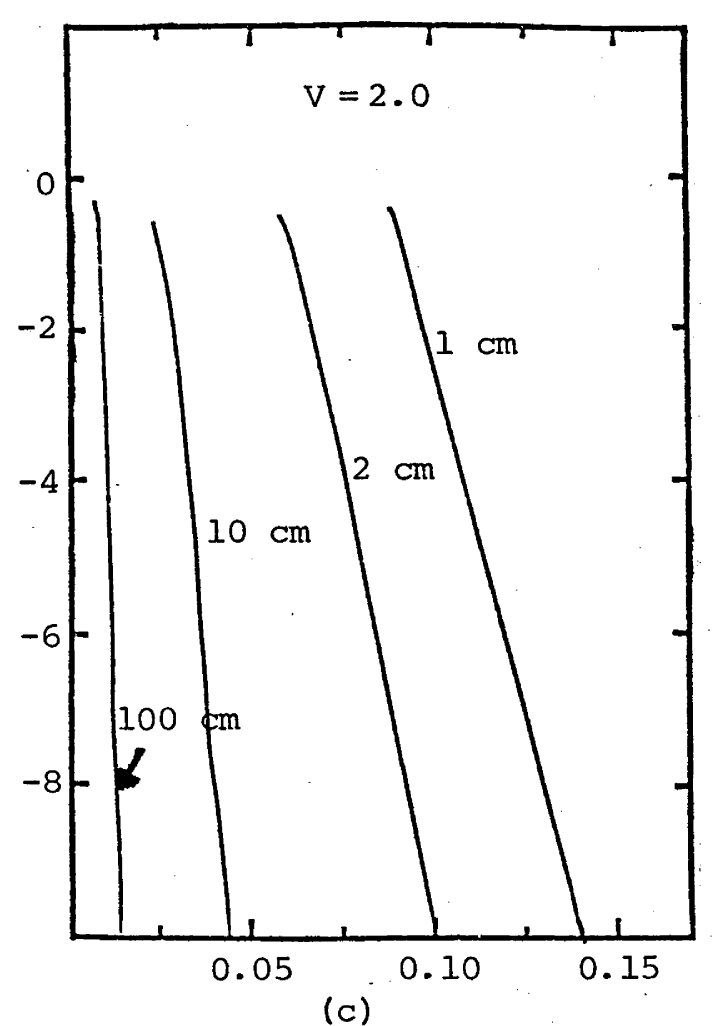

$\theta_{\mathrm{C}}$ (rads)

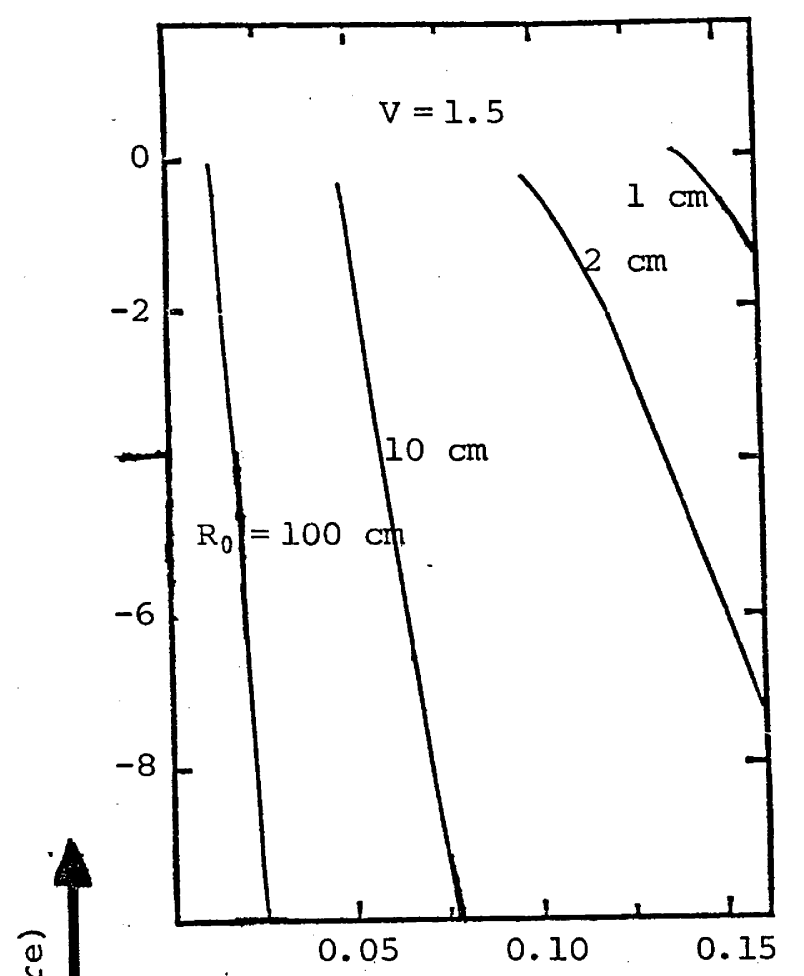

(b)

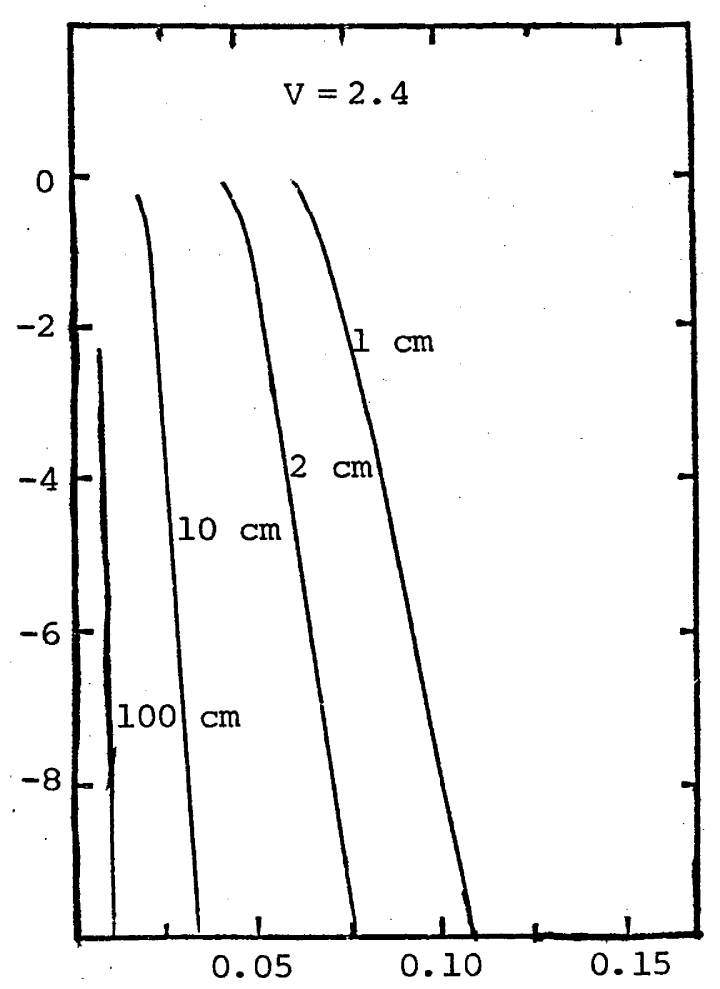

(d)

Fig. 8: Plot of bending losses in decibels/metre for four normalised frequencies, for a waveguide with a core radius of $2 \mu\left(2 \times 10^{-6}\right.$. metres). 
which for a $10 \mathrm{~cm}$ bend and $\theta_{c} \sim 0.05$, and the parameters of fig. 7, this ratio becomes approximately 10 .

The increased loss of the slab waveguide in comparison to the optical fibre with identical waveguide parameters (e.g. same angle of inclination, $\theta_{z}$, to the waveguide, i.e. $\beta=k_{1} \cos \theta_{z}$ ) can be explained by the fact that the mode in the round fibre experiences varying radius of curvature of the interface at different points in the cross section of the bent fibre. In the plane of the bend, the mode experiences a curvature $\left(R_{0}+\rho\right)$ of the surface, whereas in the plane perpendicular to the bend, the radius of curvature surface is $\infty$. From the generalised Fresnel's law, the radiation loss will thus vary from zero in the plane perpendicular to the bend to a finite maximum near the plane of the bend, as discussed previously. Thus, the dominant contribution to the total radiated power comes from the part of the mode in or near the plane of the bend. The slab waveguide mode experiences a constant radius of curvature at the outer cladding interface and thus all the active radiation surface contributes equally to the total radiated power.

When the ${ }^{L P}{ }_{\ell m}$ mode formulation is used to determine the curvature induced losses, the general LP $\ell_{m}$ modes $(l \neq 0$, where $l=0$ corresponds to the $\mathrm{HE}_{1 \mathrm{~m}}$ modes) behave differently due to a bend in the waveguide axis, in comparison to the $\mathrm{HE}, \mathrm{EH}$ modes. The two polarisations of the LP modes suffer radically different radiation losses depending on the orientation of the mode to the plane of bending $[15-16,12]$. This can be understood by reverting to the heuristic discussion of the introduction. In the $\mathrm{EH}, \mathrm{HE}$ mode formulation, the intensity distribution of the guided power about the axis of the fibre is uniform. However, the LP modes have a non-uniform intensity distribution about the fibre axis as illustrated in fig. 9. As the active region, for which the dominant radiation occurs, is located at or near the plane of the bend, one can readily observe that the radiation loss of the $\mathrm{EH}, \mathrm{HE}$ modes is unaffected by the orientation of the plane of the bend, since there is always the same intensity distribution about the plane of the bend. However, from the intensity distribution of the LP modes, fig. 9, it can be seen that as the orientation of the plane of bending is varied relative to the orientation of the LP mode, the intensity of the mode in 


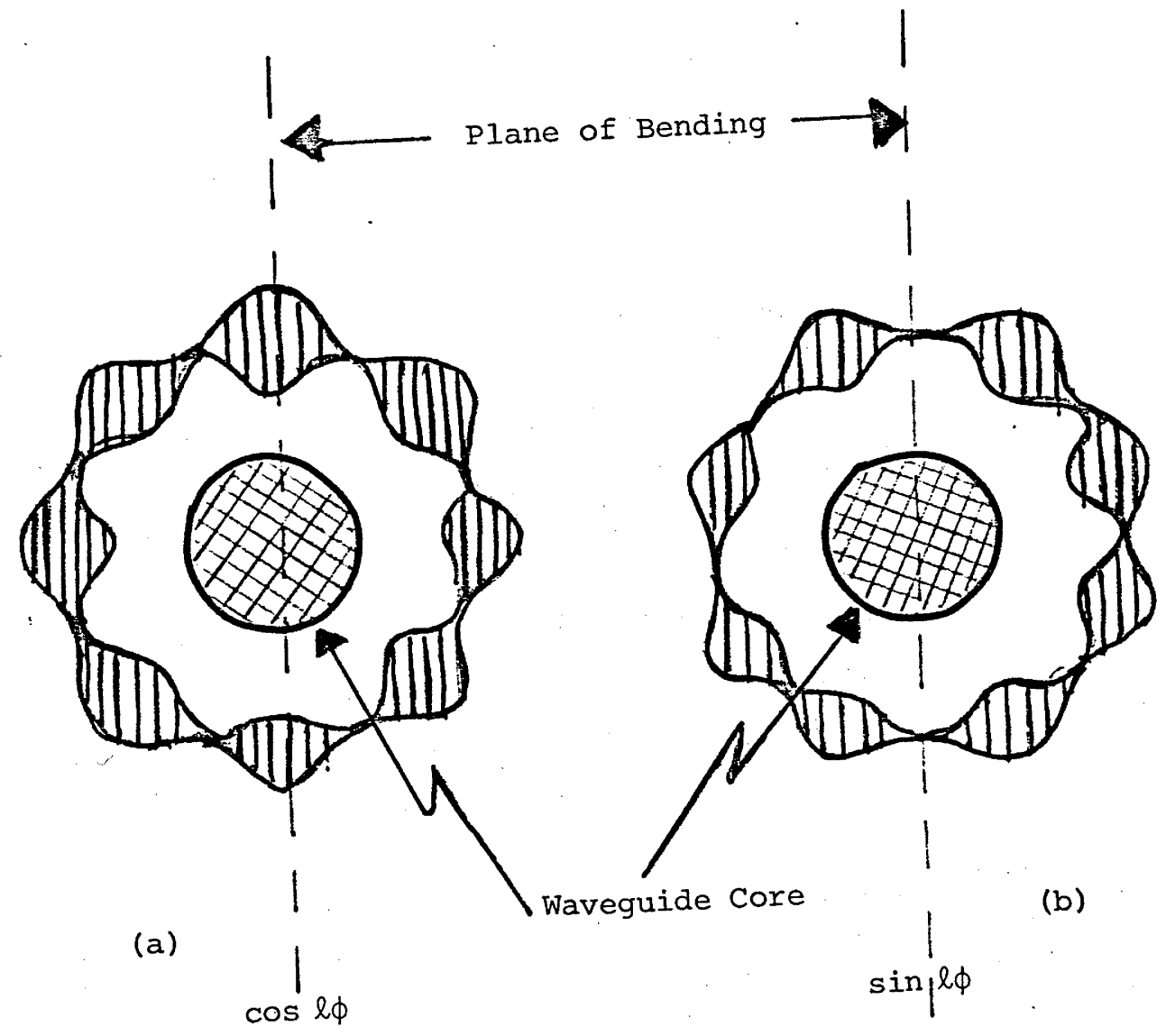

Fig. 9: The azimuthal variation of the intensity of the LP modes of the dielectric optical fibre. 
the active radiating region of the plane of the bend varies so that the radiation loss varies. The radiation loss can vary from a maximum when the plane of the bend is coincident with a plane of maximum intensity to zero when the plane of the bend is in a null zone of the intensity distribution. As the mode number of the LP mode increases, the intensity distribution of the modes tends to become more and more uniform so that we should expect that for higher order IP modes, this polarisation sensitivity in bends decreases. As the analysis of ref. 12 was not applicable to this situation, the truth of this statement has not yet been confirmed.

Due to the difficulty of measuring the radiative losses due to bends in optical fibres, the experimental verification of equation (17) has not yet been provided with any degree of confidence. The experimental results of Kapron et al. [45] confirm the dominant exponential behaviour of the attenuation coefficient but do not compare the magnitude of the losses with that predicted by the extant theories of the time. The most important studies on bending losses of monomode dielectric waveguides are those of Neumann and Rudolf [27] for optical fibres in the microwave range. However, the magnitude of the radiative losses can not be taken as conclusive proof, since the optical fibres used were of large dielectric differences between the core and cladding. From our heuristic discussion of the bending losses of optical waveguides, we have seen that by increasing the acceptance angle of an optical waveguide, $\theta_{C}\left[=\sin ^{-1}\left(1-\varepsilon_{2} / \varepsilon_{1}\right)^{\frac{1}{2}}\right]$, the radiation loss is reduced; thus, we may expect, as can be seen from the results of ref. 27 , that the measured attenuation coefficient is much less than that predicted by equation (17). In fig. 7 of ref. 27, the measured attenuation coefficient is plotted and compared with the theoretical predictions extant at this time. The figure illustrates this discrepancy in the magnitude of the loss in comparison to the theoretical results, discussed above, as well as confirming that the result of equation (17), which agrees with equation (27) of ref. $27,{ }^{\dagger}$

\footnotetext{
+ Equation (27) of ref. 27 is purported to be Lewin's original curvature loss result for optical fibres, which was in error by a factor of $2(11,10]$. However, due to a misinterpretation of the cmplitude attenuation coefficient of the fields in the bend, equation (8) of ref. 7 , as the power attenuation coefficient, equation (27) is in fact correct.
} 
provides the best fit for the variation of the attenuation coefficient.

In this analysis, we have ignored the effect of field deformation of the incident mode as it propagates around the bend. Marcuse's numerical analysis [13] of this situation indicates that the effect of field deformation is minimal for bending radii, with an aspect ratio

$$
\begin{gathered}
\frac{R_{0}}{\rho}>500 \quad \text { for } a \quad V=2.4 \text { fibre } \\
\text { and } \theta_{C}=0.14
\end{gathered}
$$

From the condition for the applicability of the conventional modal power attenuation coefficient ignoring all field deformation, equation (20), we have the criteria that

$$
\frac{R_{0}}{\rho}>\frac{V^{2}}{2 w \theta_{C}^{2}}
$$

from equation (19) for a $v=2.4, \theta_{C}^{2}=0.02$, as used in Marcuse's analysis. This yields

$$
\frac{R_{0}}{\rho}>80
$$

The difference between the two bending aspect ratios does not appear to be sufficiently large to assume that equation (17) is valid for $R_{0} / \rho \sim 500$, for the fibre with $V=2.4$ and $\theta_{C}^{2}=0.02$. As the lower limit on the aspect ratio of the bend, $R_{0} / \rho$, arises from the approximation

$$
\exp \left\{\frac{\rho}{R_{0}} \cdot \frac{v^{2}}{2 w \theta_{c}^{2}}\right\} \sim 1
$$

we can easily test the validity of the above statement.

and

$$
\begin{aligned}
& \text { For } \mathrm{R}_{0} / \rho \sim 500, \\
& \qquad \frac{\mathrm{v}^{2}}{2 \mathrm{~W} \theta_{\mathrm{C}}^{2}} \sim 80
\end{aligned}
$$

$$
\begin{aligned}
\exp \left\{\frac{\rho}{R_{0}} \cdot \frac{V^{2}}{2 W \theta_{C}^{2}}\right\} & \sim e^{1 / 6} \\
& \sim 1.16 .
\end{aligned}
$$


Thus at $\rho / R_{0} \sim 500$, the conventional modal attenuation coefficient from equation (17) already has an inherent 16\% error. In order to understand this field deformation of the incident modal field, we can consider the geometric optics of rays propagating in a bent waveguide, as in the following section.

\subsection{RAY OPTICAL DETERMINATION OF THE CRITICAL BENDING RADIUS}

In the preceding analyses of the radiative loss from a mode incident on a bend in the optical fibre, we have made two assumptions:

(1) The structure of the field is not altered by the bend in the waveguide, i.e. there is no deformation of the field; and

(2) The neglect of coupling between the incident mode and other bound modes of the waveguide.

The study of these effects has received little attention [13, $18,41]$ and to the author's knowledge, no simple explanation has ever been provided for them.

In this section, we discuss these effects for the particular case of step-index waveguides and present a geometric optics explanation of them, in order to derive an order of magnitude estimate for the ratio, $\rho / R_{0}$, for which these effects can be ignored, and thus the foregoing analysis is valid.

We will begin with the consideration of the deformation of the field and in order to understand more fully the physical processes, we will concern ourselves with slab waveguides. If we consider the situation, as illustrated in fig. 10, in which the family of rays that compose the mode of the straight slab waveguide with propagation constant $B$ such that

$$
\beta=k_{1} \cos \theta_{z}
$$

are incident on a curved section of waveguide, each of the rays in the family are incident on the outer and inner core-cladding interfaces at 

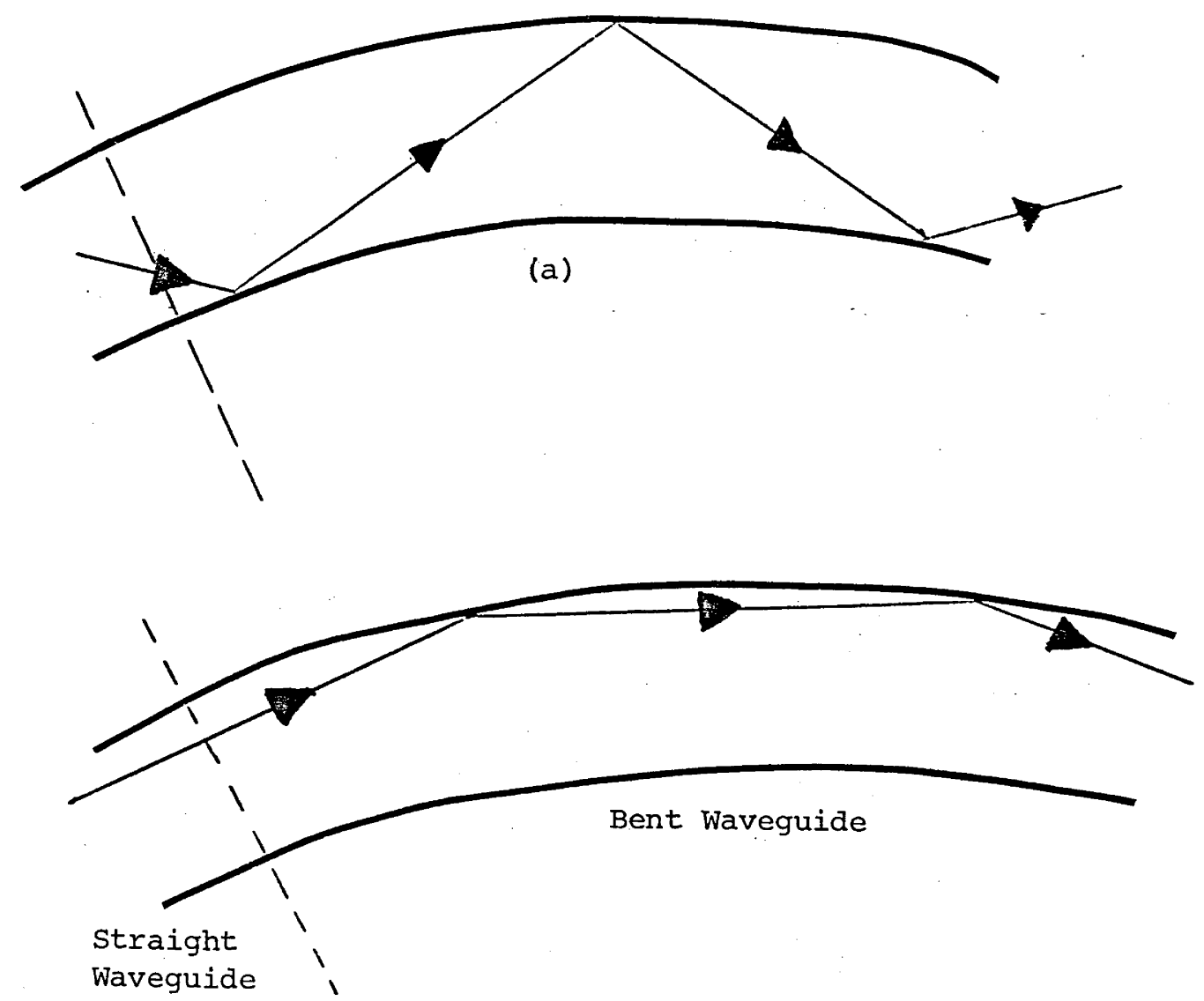

(b)

Fig. 10: A schematic drawing of the propagation of rays around a bent waveguide. The ray can be entirely guided by only the outer corecladding interface as in fig. $10 \mathrm{~b}$. 
different angles. If the radius of curvature of the axis of the waveguide is large enough, all the rays in the family are reflected from both the outer and inner core-cladding interfaces as in fig. 10a. However, as the radius of curvature is reduced below a critical radius $R_{C}$, some of the rays are only reflected from the outer core-cladding interface, as in fig. lob. This critical radius, $R_{C^{\prime}}$ is the radius of curvature of the axis for which the outermost ray (relative to the centre of the curvature) of the straight waveguide has an inner caustic in the bent segment with a radius of curvature greater than the radius of curvature of the inner core-cladding boundary.

For radii of curvature below this critical radius, the intensity of rays in the cross section of the bent waveguide is greatly different from intensity distribution of the straight waveguide as can be seen from fig. 11. This intensity redistribution is the geometric optics equivalent of the deformation of the electromagnetic field. From fig. 1l, we observe that the modal field deformation results in a narrowing of the field toward the outer core-cladding interface in the waveguide cross section as reported by Marcuse [13].

The rays incident on the curved segment of the waveguide closest to the outer core-cladding interface will be the first to experience this effect since they are incident on the curved outer corecladding interface in the bent waveguide at the smallest angles. As the radius of curvature is decreased, more and more of the rays in the family that constitute the mode will be similarly affected. We hypothesise that "the condition for the significant distortion of the modal field due to a bend in the waveguide is when all or part of the mode ceases to be guided by the two core-cladding interfaces." In ray terminology this condition means that when one of the rays that make up the mode ceases to be totally internally reflected by both core-cladding interfaces, then we can consider there to be significant deformation of the modal field. This corresponds to the outermost ray of the family of rays of the mode that is incident in the curved section of waveguide, having an inner caustic, in the bend, with a radius greater than the radius of the inner core-cladding boundary, i.e.

$$
\left(R_{0}+d\right) \cos \theta_{z}>\left(R_{0}-d\right)
$$




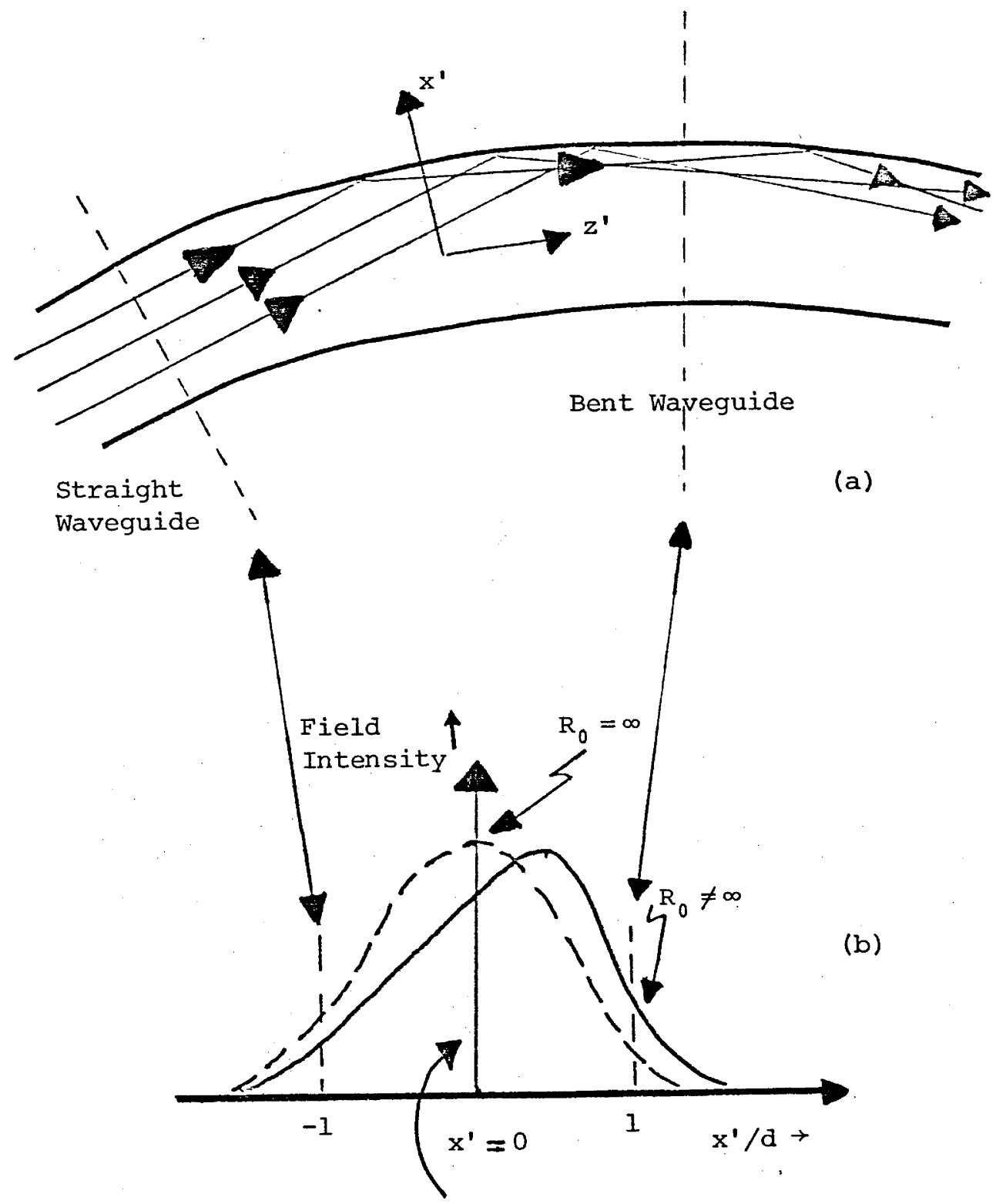

The Waveguide Axis

Fig. 11: The geometric optics interpretation of the deformation of a field in a bend. In fig. (b), the cross sectional field intensity of the incident mode is plotted for the two situations of a straight waveguide (dotted line) and a bent waveguide (solid line). 
So that for a radius curvature with $R_{0} / d \gg 1$, we can write, using the transformations of ray to modal parameters via equation (3.25), the aspect ratios below the critical aspect ratio, $\mathrm{R}_{C} / d$, at which one can consider significant distortion of the modal field of the straight waveguide to occur, satisfy

$$
\frac{R}{d} \leqslant \frac{R_{C}}{d}=\left(\frac{2 V}{U \theta_{C}}\right)^{2}
$$

Thus, equation (3) provides a quantitative limit on the aspect ratio, i.e. $R_{0} / d$, for which the incident modal field can be considered to be a valid approximation to the field in the bent slab waveguide. Although the criteria is somewhat arbitrary due to the choice of ray to which we apply the criteria, this should provide a good estimate of the applicability of the approximations used in the previous analysis for the slab waveguide.

From equation (3), we can see that this criteria cannot be satisfied for a particular waveguide for a radius of curvature greater than the critical radius of curvature

$$
\frac{\mathrm{R}_{\mathrm{C}}}{\mathrm{d}}=\left(\frac{2 \mathrm{~V}}{\mathrm{U}{ }_{\mathrm{C}}}\right)^{2}
$$

and for such bends we can use the approximation of no field deformation with confidence.

Also from equation ( 3 ), it can be readily observed that the modes far from cutoff, i.e. $\theta_{\mathrm{z}} \rightarrow 0$, or equivalently $(\mathrm{U} / \mathrm{V}) \rightarrow 0$, are more. susceptible to field deformation as is observed [13].

Although the above analysis is strictly applicable to the slab waveguide, these results can be used to provide a lower limit on the aspect ratio, $R_{0} / \rho$, of bends in optical fibres. Due to the inherent curvature of the core-cladding interface in round dielectric optical fibres, skew rays (i.e. the rays corresponding modes with $\ell>1$ ) can never be guided entirely by the outer part of the core-cladding interface, as can be seen from fig. 12 (i.e. the fraction of the surface furtherest away from the centre of curvature). Thus it is only the low order modes of the bent optical fibre which are deformed significantly 


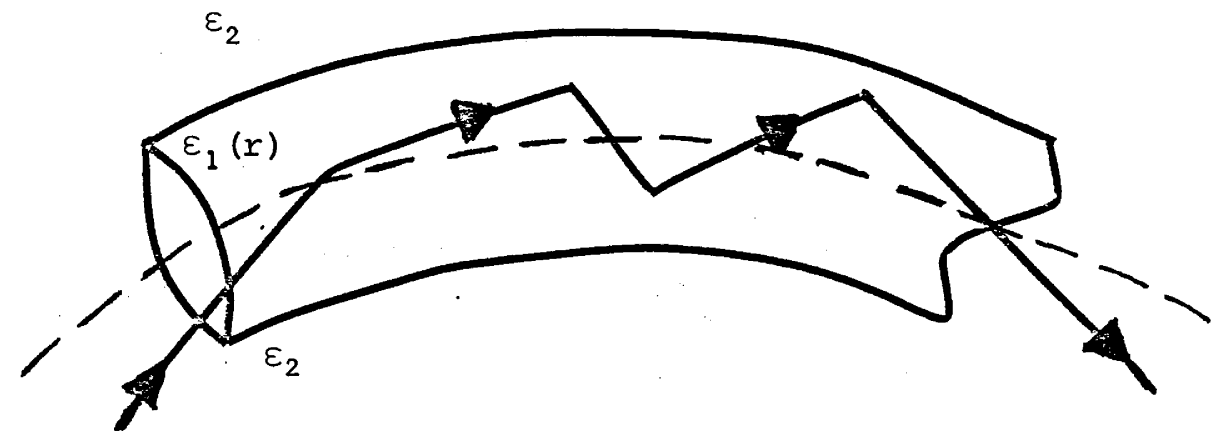

(a)

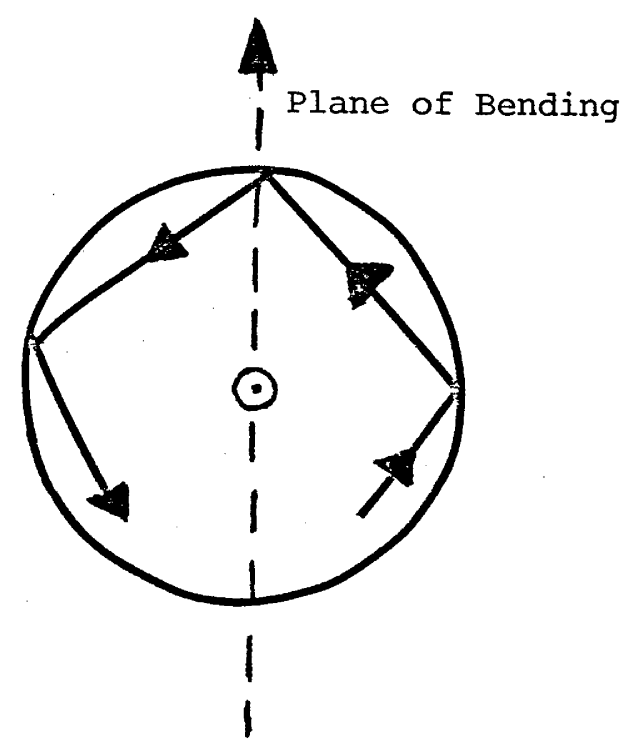

(b)

Fig. 12: The propagation of a ray along a round optical fibre with a bend in the waveguide axis. Due to the inherent curvature of the core-cladding interface, in (b), the ray cannot ever be totally guided by the outermost part of the core-cladding interface, as can happen in the slab. 
by curvature of the waveguide axis. Therefore, application of equation (3) to a bend in the axis on an optical fibre requires that field deformation occurs for aspect ratios, $R_{0} / \rho$, for which

$$
\frac{\mathrm{R}_{0}}{\rho} \leqslant \frac{\mathrm{R}_{\mathrm{C}}}{\rho}=\left(\frac{2 \mathrm{~V}}{\mathrm{U} \theta_{\mathrm{C}}}\right)^{2} \text {. }
$$

From Marcuse's numerical analysis [13] on a monomode stepindex optical fibre, with

$$
\theta_{C}^{2} \sim 0.02
$$

the above criteria, for a $V=2.4$ fibre, the critical aspect ratio $R_{C} / d$ becomes

$$
\begin{aligned}
\frac{R_{C}}{\rho} & =\left(0.12 \theta_{C}^{2}\right)^{-1} \\
& =420 \quad \text { for } \theta_{C}^{2}=0.02 .
\end{aligned}
$$

From the numerical results of Marcuse, we observe that for an aspect ratio $R_{0} / \rho \sim 500$, the field deformation is nearly negligible, which would appear to satisfy our criteria well.

Again, from Marcuse's calculations for a $V=12.76$ fibre, the $\mathrm{HE}_{11}$ mode by the above criteria would be expected to be deformed in a bent waveguide for aspect ratios $R_{0} / d$ below the critical ratio $R_{C} / d$, where

$$
\begin{aligned}
\frac{R_{C}}{d} & \sim \frac{170}{\theta_{C}^{2}} \\
& =8500 \quad \text { for } \theta_{C}^{2}=0.02 .
\end{aligned}
$$

Although Marcuse's analysis is limited to aspect ratios of 1000 at this upper limit, the effect of the field deformation results in a reduction in the power attenuation coefficient by a factor of 0.04 , compared to the conventional calculation, assuming no field deformation. Thus, even at an aspect ratio of $R_{0} / d \sim 1000$, there is significant field deformation. Thus it would appear that our critical aspect ratio $\mathrm{R}_{C} / \mathrm{d}$ is well justified. 
In Table 1, we calculate the critical aspect ratios for the next few modes in the $\mathrm{V}=12.76$ fibre and list Marcuse's numerically calculated change in the power attenuation coefficient due to the deformation of the incident field in the bend. From these values, it can be seen that the criteria, equation (5), provides a valuable quantitative measure of the effect of field deformation, even though it is strictly a ray optical argument developed for multimode slab waveguided.

We can extend the applicability of this geometric optics analysis to include the effect of mode coupling between the bound modes of the multimode optical waveguide due to the constant curvature of the waveguide axis. The bound modes of an optical waveguide have a discrete set of eigenvalues, $\beta$, corresponding to a discrete spectrum of ray propagation angles $\theta_{z}$ to the axis, via

$$
\beta=k_{1} \cos \theta_{z} \text {. }
$$

The angular separation of the modes in a weakly guiding waveguide can be written as

$$
\Delta \theta_{m}^{n}=\frac{U_{n+1}-U_{n}}{k_{1} \rho}
$$

since

where

$$
\mathrm{u}_{\mathrm{n}}=\mathrm{k}_{1} \rho \sin \theta_{\mathrm{n}}=\mathrm{k}_{1} \rho \theta_{\mathrm{n}}
$$

$$
\theta_{\mathrm{n}} \leqslant \theta_{\mathrm{c}} \ll 1
$$

where $\theta_{n}$ is the angle of inclination of the rays to the straight waveguide axis.

If we consider the slab waveguide configuration used previously for the field deformation, fig. 10, each family of rays that constitute a mode produces a spectrum of angles of incidence at the inner and outer core-cladding interfaces. To begin, let us consider the rays $A$ and $B$ of fig. 12a. These rays form the extremes of the angular range of angles of incidence of the family of rays at the outer corecladding interface, measured relative to the tangent ot the interface. The range of angles of incidence is, from $\theta_{n}$ for ray $A$ and $\left(\theta_{n}+\varepsilon_{0}\right)$ for 


\begin{tabular}{|cc|c|c|c|c|c|}
\hline${ }^{L} P_{\ell m}$ & \multicolumn{2}{|c|}{$\begin{array}{c}\text { Transverse Wave } \\
\text { Numbers of the Mode }\end{array}$} & $\begin{array}{c}\text { Critical Bending } \\
\text { Radius }\end{array}$ & $\alpha_{\text {deform }} / \alpha_{\text {conventional }}$ \\
\hline$\ell$ & $\mathrm{m}$ & $\mathrm{U}$ & $\mathrm{W}$ & $\mathrm{R}_{\mathrm{C}} / \rho=\left(2 \mathrm{~V} / \mathrm{U} \theta_{\mathrm{C}}\right)^{2}$ & $\mathrm{R}_{0} / \rho=500$ & $\mathrm{R}_{0} / \rho=1000$ \\
\hline 0 & 2 & 5.10 & 11.70 & 1250 & 4 & 2 \\
0 & 3 & 7.98 & 9.96 & 510 & 3 & 2 \\
0 & 4 & 10.79 & 6.82 & 120 & - & - \\
1 & 1 & 3.55 & 12.26 & 2590 & 0.02 & 0.1 \\
1 & 2 & 6.48 & 10.99 & 780 & 3 & 2 \\
1 & 3 & 9.35 & 8.68 & 370 & 0.9 & 3 \\
2 & 1 & 4.75 & 11.84 & 1440 & 0.4 & 0.5 \\
2 & 2 & 7.76 & 10.12 & 540 & 1 & 1 \\
3 & 1 & 5.9 & 11.31 & 934 & 0.3 & 0.7 \\
\hline
\end{tabular}

Table 1: The table represents the ratio of the modal power attenuation coefficient of the $\mathrm{LP}_{\ell m}$ modes calculated by including field deformation and the conventional calculations ignoring the field deformation, for a $V=12.76$ step-index fibre with acceptance angle $\theta_{C}=0.14 \mathrm{rad}$ for two bending radii, and the critical bending radius of each mode is presented, to see whether the critical bending radii have been exceeded. (As Marcuse indicates in ref. 13 the modes with smallest $U$ (or largest $\mathrm{W}$ ) tend to be more precise due to computational difficulties.) 
(a)

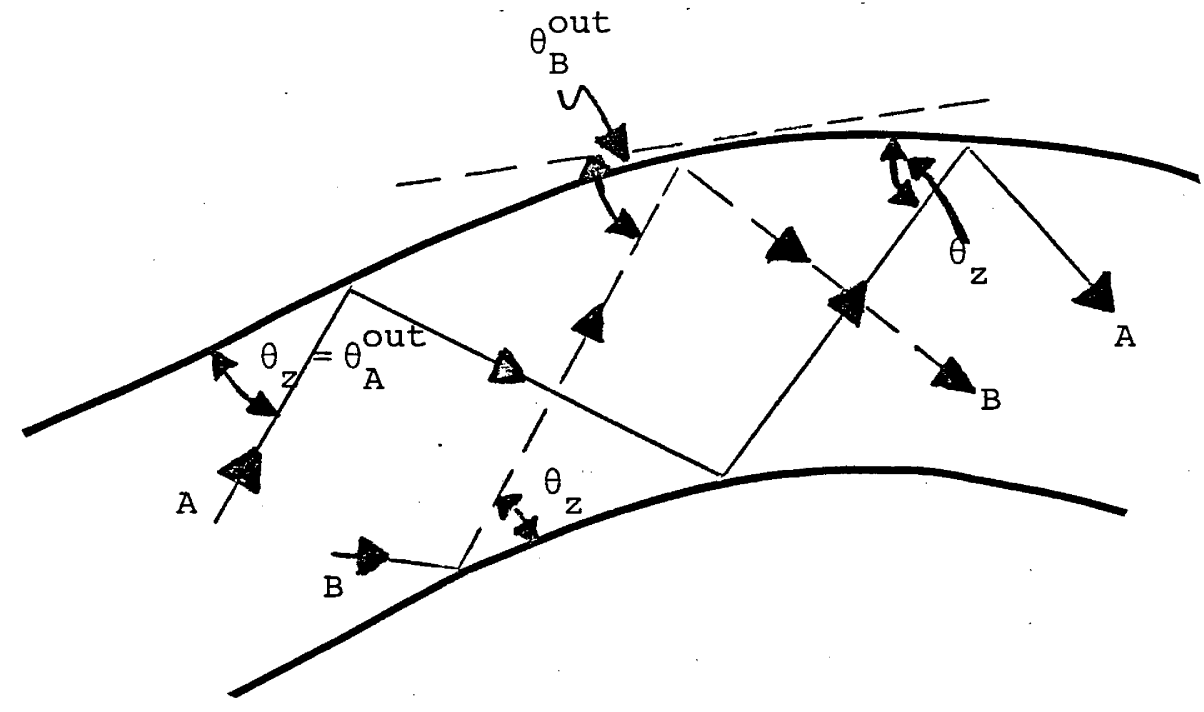

(b)

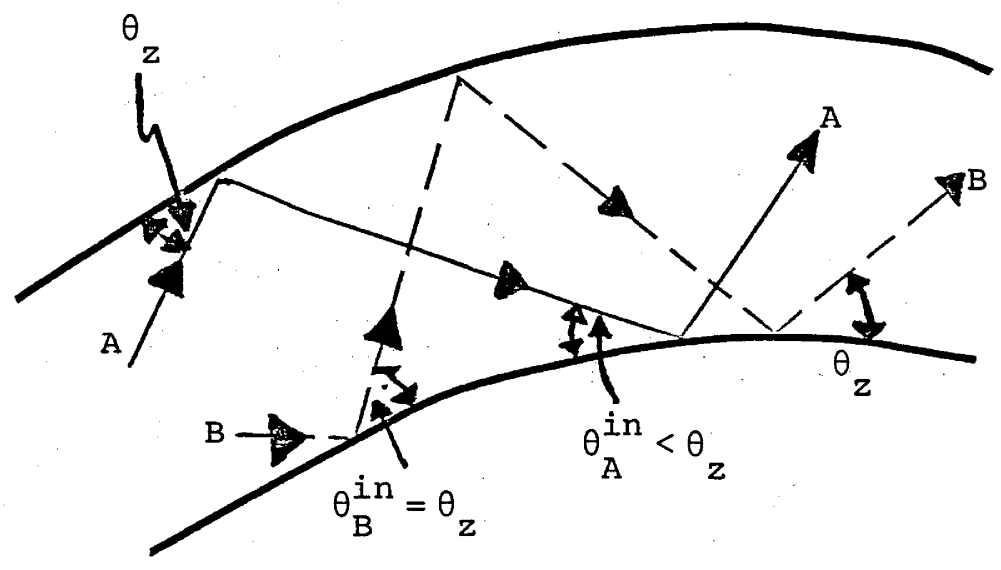

Fig. 13: (a) The two rays $A$ and $B$ of the mode of the straight slab with angle $\theta_{z}$ to the axis, that produce the minimim and maximum angles of incidence at the outer core-cladding interface, respectively.

(b) As for (a) at the inner core-cladding interface.

(c) See over page. 
(c)

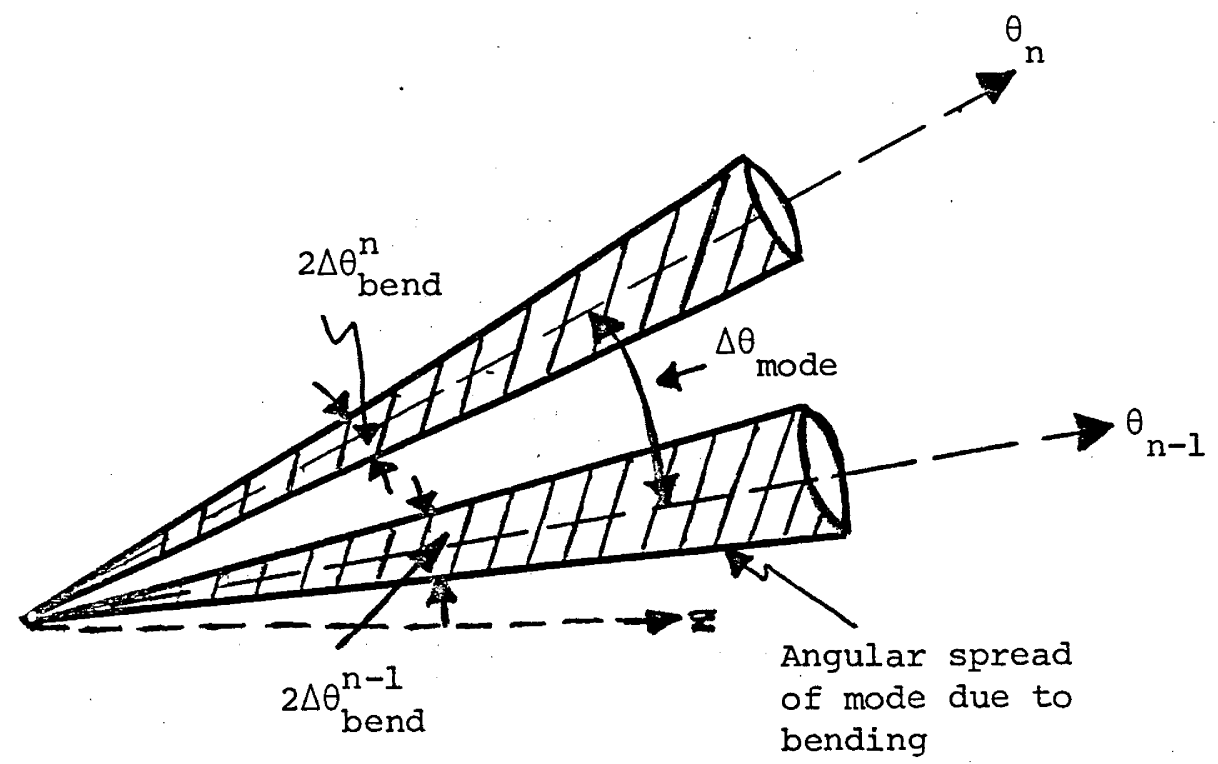

Fig. 13: (c) The angular spread, $\Delta \theta_{\text {bend }}$ of the two adjacent modes of the slab waveguide due to a bend in the axis relative to the angular spacing between the modes $\Delta \theta_{\text {mode. }}$ 
ray $B$, where for $d / R_{0}<1$

$$
\varepsilon_{0}=\frac{2 d}{R_{0}} \cot \theta_{z} .
$$

At the inner surface, consider rays $C$ and $D$, as in fig. 13b, of the same mode as the rays in fig. 13a. These rays produce the extremes in the angular range of the angles of incidence for the rays of the mode at the inner core-cladding interface, viz.

$$
\theta_{n} \text { for ray } c \text { and } \theta_{n}-\varepsilon_{i} \text { for ray } D \text {, }
$$

where $\theta_{i}=\varepsilon_{0}$ to first order in $d / R_{0}<1$

Rewriting the angular spread, $\varepsilon_{0}$, in modal terminology, equation (12) becomes, from equation (3.25)

$$
\varepsilon_{0}=\frac{2 d}{R_{0}} \cdot \frac{\beta d}{U} .
$$

Therefore, in the bent waveguide, the family of rays constituting a mode of a straight waveguide have a spread in angles relative to the tangent to the concentric circles, as in fig. 13c. Thus, if the spread in the rays of the families corresponding to two consecutive modes overlaps in the bent waveguide, these rays can ultimately become part of the other mode. In which mode family the ray ultimately finishes depends on the length of the bent waveguide, as the position in the cross section of the waveguide at the termination of the bend will determine its angle of inclination to the axis of the straight waveguide that terminates the bent section in an analogous fashion to the ray entering the bent waveguide. Therefore, by varying the length of the bend, one can vary the amount of coupling between the bound modes. This is observed in practice [42], and is predicted from the theoretical analysis of ref. 3 .

Thus, from our discussion, it is found that mode coupling becomes significant in multimode slab waveguides if the angular spread of the family of rays that constitute a mode in the bent waveguide overlaps with the angular spread of the family of rays of the next mode. From equation (10) for the angular spacing of the bound modes, we find 
that mode coupling occurs if

$$
\Delta \theta_{\mathrm{n}} \leqslant 2 \varepsilon_{\mathrm{o}}
$$

or from equation (13), using the weakly guiding fibre approximation $\beta / k_{1} \sim 1$, equation (14) becomes

$$
\frac{\mathrm{R}_{0}}{\mathrm{~d}} \leqslant \frac{4 \mathrm{~V}^{2}}{\mathrm{U}_{\mathrm{n}} \Delta \mathrm{U}_{\mathrm{n}} \cdot \theta_{\mathrm{c}}^{2}}
$$

where

and

$$
\left.\begin{array}{rl}
v & =k_{1} d \theta_{c} \\
\Delta u_{n} & =u_{n+I}-u_{n} .
\end{array}\right\}
$$

In the slab waveguide, there are $V$ modes for a waveguide with normalised frequence $V$, defined in equation (16), and these modes occupy an angular volume $\theta_{C^{*}}$. If we assume that these modes are equally spaced in the angular domain, we find that the angular spacing between modes is

$$
\Delta \theta_{\mathrm{n}}=\Delta \theta_{\mathrm{av}}=\frac{\theta_{\mathrm{c}}}{\mathrm{v}}
$$

so that

$$
\left.\begin{array}{l}
\Delta \mathrm{U}_{\mathrm{n}}=\mathrm{k}_{\mathrm{l}} \mathrm{d} \Delta \theta_{\mathrm{av}} \\
\Delta \mathrm{U}_{\mathrm{n}}=1
\end{array}\right\}
$$

and using equation (18) in equation (15), we find that the critical radius of curvature, $R_{C}^{m}$, below which mode coupling between bound modes occurs, satisfies

$$
\frac{\mathrm{R}_{\mathrm{c}}^{\mathrm{m}}}{\mathrm{d}}=\frac{4 \mathrm{v}^{2}}{\mathrm{U}_{\mathrm{n}} \theta_{\mathrm{C}}^{2}}
$$

From equation (19), we observe two things immediately:

(1) For a fixed radius of curvature, the mode coupling decreases as the normalised frequency of the waveguide is decreased due to the increased angular spacing between the modes; and

(2) Higher order modes are less susceptible to mode coupling as 
$\mathrm{U}_{\mathrm{n}} / \mathrm{V} \rightarrow \mathrm{l}$ as a mode approaches cutoff. From the gometric optics argument, it is seen that these modes, with rays with larger angles $\theta_{z}$ to the waveguide axis, do not spread as much as the lower order modes.

As for the field deformation argument previously, this result, equation (19), can be immediately applied to the round optical fibre. since most waveguide parameters of the optical fibre are relatively insensitive to $l$, the azimuthal mode number, in comparison to variations in $\beta$, the longitudinal propagation constant, it is this mode coupling that is significant, and equation (19) provides a good order of magnitude estimate of the aspect ratio $R_{0} / d$ below which mode coupling in multimode fibres is important.

For the $\mathrm{V}=12.76$ waveguide of Marcuse's field deformation analysis, the critical bending radius for mode coupling from the $\mathrm{HE}_{11}$ mode, from equation (19), becomes

$$
\left.\begin{array}{rl}
\frac{R_{C}}{\rho} & =\left(\frac{2.9 \times 10^{2}}{\theta_{C}^{2}}\right) \\
& =1.45 \times 10^{4} \quad \text { for } \theta_{C}^{2}=0.02,
\end{array}\right\}
$$

i.e. mode coupling occurs at a bending radius twice as large as the bending radius at which field deformation becomes significant, from equation (9). This study supports that of Marcatili's [1] in which he demonstrates that the coupling between bound modes is more significant than the radiative loss from the mode in a multimode optical waveguide.

The above geometric optics analysis of field deformation and mode coupling due to bends in the optical waveguide has provided physical insight into the processes as well as providing a reasonably accurate quantitative estimate of the regimes of applicability of the analysis by the Surface Current Method. The above analysis however, suffers from the disability that it does not provide a quantitative estimate of the corrections to the analysis when the curvature of the waveguide is less than the critical radius of curvature. 


\subsection{CONCLUSIONS}

In this chapter, we have used the Surface Current Method to determine the power radiated from a bound mode of a dielectric optical waveguide due to bends in the waveguide axis of a constant radius of curvature. Although the application of the Surface Current Method is lengthy, the analysis is straightforward and provides much physical insight into the structure of the radiation field due to the bend. The use of the Surface Current Method circumvents the necessity of a spectral representation of the radiation field, necessary in most other analyses. From the analysis of the tunnelling leaky modes of the straight dielectric optical fibre and the radiation losses due to bends in optical fibres, we observe that no additional analysis is required for the calculation of the graded-index fibre power attenuation coefficients in comparison to the analysis of the step-index optical fibre. The only discrepancy between the two results in both examples is the power normalisation coefficient of the modes of the waveguide, $A_{\ell \mp 1}$ ' defined in equation $(2.2 .53)$.

Although we have only analysed the radiation losses of the fibre modes for planar bends of constant radius of curvature, the form of the attenuation coefficient, equation (4.17), with its strong exponential dependence on the radius of curvature of the bend, allows us to estimate the radiative loss due to curvature for a much wider range of curvature phenomena. For a random planar bend in the axis of the waveguide, order of magnitude estimates which will provide the ultimate limits of the bending loss due to radiation can be determined by using the upper and lower bounds on the radius of curvature of the randomly bent fibre. From these two radii, the minimum and maximum radiative losses occur for the optical fibre. Since the radiation losses are dominated by the radiation from the bend in the waveguide for which the radius of curvature is least, an estimate of the average attenuation, $\alpha_{a v^{\prime}}$ of the length $L^{\prime}$, of randomly bent fibre, for a length $L_{m}$ of fibre with the minimum radius of curvature can be written as

$$
\alpha_{a v}=\frac{\alpha\left(R_{m}\right) L_{m}}{L^{\prime}}
$$


The more practical problem of non-planar bends in the optical fibre has been studied by Marcuse [14]. However, from the analysis of this chapter using the ray optical interpretation of the azimuthal travelling wave modes, his result that the loss is basically equivalent to the planar bend, with the radius of curvature replaced by the local radius of curvature of the helix, does not appear as obvious as one might at first imagine. From a consideration of the two classes of rays that make up the azimuthal standing wave modes, it can be seen that even for large radius of curvature of the helical structure, these two families of rays no longer experience the same radius of curvature in the plane of incidence. Even though the difference in radius of curvature will be slight for large aspect ratios, due to the dominant dependence of the radius of curvature of the attenuation of the bound mode, these two families of rays appear to suffer different radiative losses. The magnitude of this apparent discrepancy needs to be determined from the application of the surface Current Method. We shall mention this again in the final chapter.

Finally, in the analysis of the bend radiation, we have used three major assumptions:

(1) The deformation of the field of the mode due to the bend is negligible.

(2) The effects of elastic deformation of the dielctrics and the deformation of the waveguide cross section are negligible.

(3) The incident mode of the straight optical fibre coupled predominantly into one mode of the bent structure with mode number $m=\beta R_{0}$, around the waveguide axis.

From the analysis of section 5, we have derived limits on the aspect ratio, $\mathrm{R}_{0} / \rho$, for bends in step-index optical waveguides for which the assumptions concerning the lack of field deformation are justified. Although the criteria used are somewhat arbitrary, we have shown that the criteria do provide a reasonable estimate of the allowed bending losses. In most practical situations for bends in monomode waveguides, the material tolerance to bending becomes the significant factor in determination of the minimum bending loss for which the foregoing 
analysis is applicable rather than the field deformation, since, for the radius of the optical waveguides used in fig. 8 , i.e.

$$
\rho=2 \mu
$$

the critical bending radius of the monomode fibre, $R_{C}$, with $V=2.4$, and $\theta_{C}=0.14$ (as in Marcuse's analysis [13]) from equation (5.5), is

$$
\begin{aligned}
R_{C} & \sim\left(500 \times 2 \times 10^{-6}\right) \text { metres } \\
& \sim 1 \mathrm{~mm},
\end{aligned}
$$

i.e. the radius of the bend in the waveguide at which field deformation occurs is well below the material tolerances of the glass fibre.

The second assumption in these calculations has been studied, in part, by Hannay [43], in which the effect of the elastic deformation of a straight dielectric optical waveguide of planar cross section is determined. In Hannay's analysis, the deformation of the straight waveguide into the bent waveguide causes compression and expansion of the dielectric materials about the radius of curvature of the axis. This induces an extra dielectric profile, which when one considers the effective dielectric profile due to a bent uniform step-index slab waveguide, as in fig. 14, is in the opposite direction to the curvature induced profile. Thus, the radiative loss will be reduced, and as Hannay demonstrates, this can be accounted for by an effective radius of curvature larger than the actual physical bend radius. However, as this study is for planar waveguides, the extent of this effect in waveguides of finite cross section has yet to be determined.

From the comparison of the radiation loss measurements in fig. 7 of ref. 27 for bends in dielectric optical fibres, it can be seen that the radiative loss from the monomode waveguides is consistently less than that predicted by the conventional radiation loss formulae. This elastic deformation therefore provides another factor to consider in the explanation of this discrepancy.

The effect of the change in the waveguide cross sectional shape due to the bends inducing an elliptical cross section in the bent section of the optical fibre may predetermine the choice of modes used 


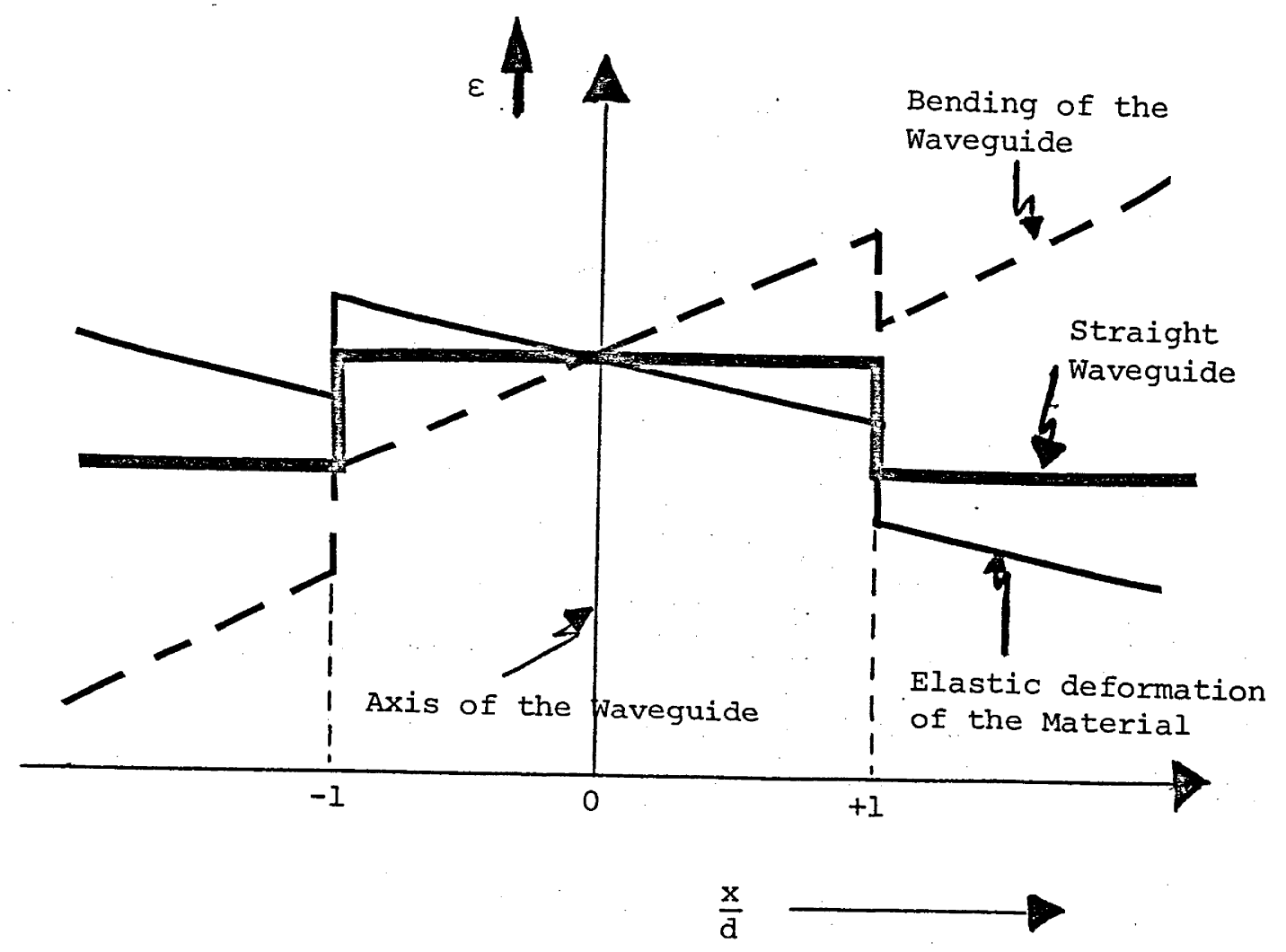

Fig. 14: The effect of bending a dielectric waveguide: the bend itself can be considered as an induced dielectric profile, together with the effect of elastic deformation of the material. 
for the calculations of bending losses. In his analysis, Marcuse [1214] used the LP "modes" to determine the radiation loss. As discussed in section 4 for a round optical fibre, these "modes" are not sufficiently stable to justify the use. However, Snyder, recently, has shown that a slight ellipticity of the waveguide cross section is sufficient to make such a linearly polarised set of modes extremely stable [44], and thus it may be necessary to study this structural deformation of the waveguide cross section to determine whether the induced ellipticity is sufficient to linearly polarise the incident fields.

The third approximation cannot be answered satisfactorily by the analysis of the Surface Current Method, but requires a detailed analysis of the electromagnetic modes of the bent structure and their coupling efficiencies to the modes of a straight optical fibre. Chang and Kuester's study [15] employing the concept of phase matching of the straight fibre mode to one toroidal mode along the path of the "centre of energy" is conceptually pleasing, and allows many analogies to be drawn from the classical mechanics analogue of a "centre of mass" of a system of masses. This concept and its mechanical analogues provide a better understanding of the physical nature of this approximation.

Although we have been able to use this concept to derive the radiation losses of straight waveguide modes incident on bent optical waveguides, we must be left with a certain degree of scepticism as to the validity of these results for the multimode fibre. From the classical mechanical analogue, the concept of a "centre of mass" of a system of bodies is useful only when the distribution of the masses about this point is strongly localised about this centre of mass, so that the entire collection of masses and their evolution in time can be characterised by the summed mass, at the centre of mass. In this electromagnetic analysis, the fields of the incident mode are not strongly localised around the "centre of energy" in comparison to the critical dimensions of the waveguide and thus the validity of this phase matching procedure as stated previously is highly questionable. We can understand this more fully from a geometric optics viewpoint. 
optical waveguide, we demonstrated that the magnitude of the radiation loss due to bends of each component of a mode, i.e. each ray of the family of rays that constitute the mode, critically depends on the location of this component in the waveguide cross section at the beginning of the bend, as can be seen from equation (3.24). Thus, from this ray optical interpretation, it would appear that the attenuation of a mode cannot be adequately characterised by one toroidal or annular mode in multimode waveguides. Although this result has used the ray family concept of a mode, without consideration of the intimate phase relationships between the component rays, and is therefore of doubtful accuracy, it does illustrate the fact that the components of the mode behave differently in the bend, depending upon their position in the waveguide cross section at the beginning of the bend.

Furthermore, for higher order modes for which little of the guided power propagates near the "centre of energy" of the mode on the fibre axis, it does not appear to be a particularly useful classification of the variation of the modal phase around the bend.

Apart from the above geometric optics analysis of section 3 for each individual ray in the multimode optical fibre, the author cannot at the present time present a consistent mathematical analysis to circumvent the above difficulties. Furthermore, even if the ray optical analysis proves to be exact, it becomes extremely tedious to accomplish for all waveguides of finite cross section, due to the difficulty in tracing the ray paths in the bent fibre. 


\section{APPENDIX A \\ RADIATION FROM THE BENT SYMMETRIC \\ SLAB WAVEGUIDE}

In this appendix, we will derive the power attenuation coefficients of the even TE modes of a step-index slab waveguide due to the radiative loss induced by a bend in the waveguide axis. To ensure that we isolate the curvature loss from other radiative mechanisms, such as the radiation due to a change in the curvature of the axis, we choose a complete annulus formed by the slab waveguide as in fig. $2 \mathrm{a}$. For a radius of curvature of the axis that is large in comparison to the cross sectional dimensions of the waveguide, we can use the philosophy of section 2 to replace the field on the bend by the incident field, and matching the phase change along the axis, we find that the mode number around the axis of the annulus, $m$, i.e. $\exp \left\{-i m \phi^{\prime}\right\}$ variation, satisfies

$$
m=\beta R_{0}
$$

and the fields in the annulus evaluated at the core-cladding interface in the local cartesian co-ordinate system, as in fig. 3, are [25]

and

$$
\begin{aligned}
& E_{y^{\prime}}=\cos U e^{-i m \phi^{\prime}} \\
& H_{X^{\prime}}=-\left(\frac{\varepsilon_{2}}{\mu}\right)^{\frac{1}{2}} \frac{\beta}{k_{2}} \cos U e^{-i m \phi^{\prime}}
\end{aligned}
$$

$$
\mathrm{H}_{\mathrm{z}^{\prime}}=-\left(\frac{\varepsilon_{2}}{\mu}\right)^{\frac{1}{2}} \frac{\mathrm{iU}}{\mathrm{k}_{2} \mathrm{~d}} \sin \mathrm{U} \mathrm{e}^{-i m \phi^{\prime}},
$$

where $\mathrm{k}_{2}$ is the wavenumber in the cladding

$$
\mathrm{k}_{2}=\omega\left(\mu \varepsilon_{2}\right)^{\frac{1}{2}}
$$

where $\varepsilon_{2}$ is the dielectric permittivity of the cladding. The eigenvalue 
of this local even $\mathrm{TE}$ mode is

$$
\frac{\mathrm{W}}{\mathrm{U}}=\tan \mathrm{U},
$$

where $U$ and $W$ are the transverse wave numbers of the mode in the core and cladding respectively, defined as

$$
\mathrm{U}=\mathrm{d}\left(\mathrm{k}_{1}^{2}-\beta^{2}\right)^{\frac{1}{2}}
$$

and

$$
\mathrm{W}=\mathrm{d}\left(\beta^{2}-\mathrm{k}_{2}^{2}\right)^{\frac{1}{2}}
$$

From the symmetry in the $\phi^{\prime}$ direction around the annulus, the radiation will be symmetric around the annulus, so that we need only calculate the radiation field at one position in the azimuthal direction. For convenience, we choose this direction to be $\phi=\pi / 2$, so that

$$
\underset{\mathrm{R}}{\sim} \hat{\underline{\mathrm{Y}}}
$$

and the position vector of the surface ${\underset{\sim}{\prime}}^{\prime}$, is

$$
\stackrel{R}{\sim}^{\prime}=R_{0}+\underline{\sim}^{\prime},
$$

where $R_{0}$ is the position vector of the axis of the slab waveguide and $\underline{\sim}^{\prime}$ is the position vector of the outer core-cladding interface, relative to the axis. Writing ${\underset{\sim}{\sim}}_{0}$ and $\underline{\sim}^{\prime}$ in the global cartesian co-ordinates, relative to the centre of curvature,

$$
\stackrel{R}{\sim}^{\prime}=\left(R_{0}+d\right) \cos \phi^{\prime} \underset{\sim}{\hat{x}}+\left(R_{0}+d\right) \sin \phi^{\prime} \underset{\sim}{\hat{y}} \cdot
$$

From equations (4) and (6)

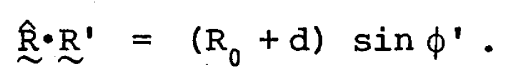

Now, from equation (5.2.2), the pseudo surface electric and magnetic currents on this core-cladding interface are

$$
\left.\begin{array}{l}
\underset{\sim}{\mathrm{K}}=-\underset{\sim}{\hat{\mathrm{n}}} \times \underset{\sim}{\mathrm{H}} \\
\underset{\sim m}{\mathrm{~K}}=\underset{\sim}{\hat{\sim}} \times \underset{\sim}{\mathrm{E}}
\end{array}\right\}
$$

and using $\underset{\sim}{\hat{n}}={\underset{\sim}{x}}^{\prime}$, and translating the currents to the global cartesian co-ordinates via 


$$
\begin{aligned}
& \hat{\underline{y}}^{\prime}=-\hat{z} \\
& \underline{\underline{z}}^{\prime}=-\sin \phi^{\prime} \underline{\sim}_{\underline{x}}+\cos \phi^{\prime} \underline{\sim}^{\hat{y}},
\end{aligned}
$$

equations ( 8 ) become

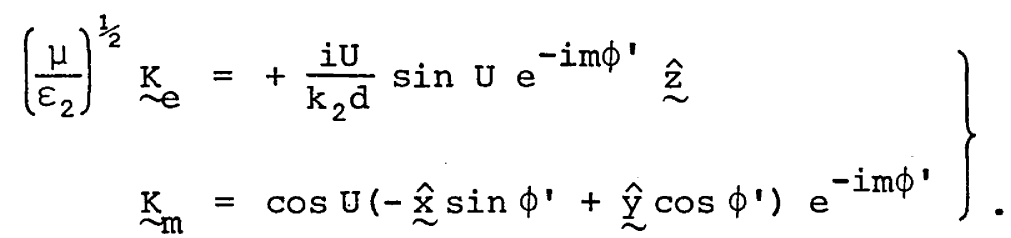

Using equation (10) in equation $(5.2 .8)$ and $(5.2 .5)$, we find we have two vector integrals to evaluate, viz.

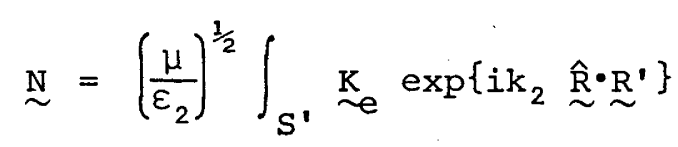

which from equations (10) and (7) becomes

$$
\stackrel{N}{\sim} \hat{z}\left(\left(R_{0}+d\right) \int_{0}^{2 \pi} d \phi^{\prime}\left\{\frac{i U}{k_{2} d} \sin U e^{i k_{2}\left(R_{0}+d\right) \sin \phi^{\prime}-i m \phi^{\prime}}\right\}\right)
$$

and

$$
\stackrel{L}{\sim}=\int_{S^{\prime}} \stackrel{\mathrm{K}}{\mathrm{m}}_{\mathrm{m}} \exp \left\{i k_{2} \underset{\mathrm{R}^{\prime}}{\mathrm{R}^{\prime}}{ }^{\prime}\right\} \mathrm{dA} \mathrm{A}^{\prime}
$$

which from equations (7) and (10) becomes

$$
\begin{aligned}
\underset{\sim}{L}=\left(R_{0}+d\right) \cos U \int_{0}^{2 \pi} d \phi^{\prime}\left\{e^{i k_{2}\left(R_{0}\right.}\right. & +d) \sin \phi^{\prime}-i m \phi^{\prime} \\
& \left.\times\left[-\sin \phi^{\prime} \underset{\sim}{x}+\cos \phi^{\prime} \underset{\sim}{y}\right]\right\} .
\end{aligned}
$$

The integration over $\phi^{\prime}$ is recognised as the integral representation of the Bessel function of the first kind [36], i.e. $J_{m}(x)$ where

$$
J_{m}(x)=\frac{1}{2 \pi} \int_{0}^{2 \pi} \exp \{i x \sin \theta-i m \theta\} d \theta
$$

and equations (12) and (14) become

$$
\stackrel{N}{\sim}=\hat{z}\left(2 \pi \frac{i U}{k_{2} d} \sin U J_{m}\left(k_{2}\left(R_{0}+d\right)\right) \cdot\left(R_{0}+d\right)\right)
$$

and 


$$
\underline{\sim}=2 \pi \cos U\left(R_{0}+d\right)\left(i J_{m}^{\prime}\left(k_{2}\left(R_{0}+d\right)\right) \hat{x}+\frac{\beta}{k_{2}} J_{m}\left(k_{2}\left(R_{0}+d\right)\right) \hat{y}\right)
$$

so that the time averaged radiant energy flux $S^{R}$ from equation (5.2.8) becomes $^{\dagger}$

$$
\begin{aligned}
S^{R}=\left(\frac{\varepsilon_{2}}{\mu}\right)^{\frac{1}{2}} \frac{k_{2} \pi\left(R_{0}+d\right)^{2}}{4 R} \mid\left(\frac{U}{k_{2} d}\right. & \sin U_{m}\left(k_{2}\left(R_{0}+d\right)\right) \\
& \left.+\cos U J_{m}^{\prime}\left(k_{2}\left(R_{0}+d\right)\right)\right)\left.\right|^{2}
\end{aligned}
$$

and the total power radiated, $\mathrm{P}^{\mathrm{R}}$, is

$$
\mathrm{P}^{\mathrm{R}}=2 \pi \mathrm{R} \cdot \mathrm{S}^{\mathrm{R}}
$$

The power attenuation coefficient of the incident mode due to the bend is

$$
\alpha=\frac{P^{R}}{2 \pi R_{0} \cdot P_{0}}
$$

where $\left(2 \pi R_{0}\right)$ is the axial distance the mode travels around the annulus and $P_{0}$ is the power of the incident guided mode [25]

$$
\mathrm{P}_{0}=\left(\frac{\varepsilon_{2}}{\mu}\right)^{\frac{1}{2}} \frac{\beta \mathrm{d}(1+\mathrm{W})}{2 \mathrm{k}_{2} \mathrm{~W}}
$$

and with equations (18), (19) in (20), we find

$$
\alpha=\frac{S^{R}}{P_{0}} \cdot \frac{R}{R_{0}}
$$

which, upon using the eigenvalue equation, equation (3), becomes

$$
\alpha=\frac{\pi}{2} \frac{k_{2}^{2} W U^{2}}{(\beta d)(1+W) v^{2}}\left(\frac{W}{k_{2} d} J_{m}\left(k_{2}\left(R_{0}+d\right)\right)+J_{m}^{\prime}\left(k_{2}\left(R_{0}+d\right)\right)\right)^{2} .
$$

Since $\beta>k_{0}$ for the bound modes, and the order of the Bessel functions is $m$, where from equation ( 1 )

$$
m \equiv \beta R_{0}>k_{2}\left(R_{0}+d\right)
$$

\footnotetext{
+ since the bent slab waveguide is a two-dimensional structure, we must use equation $(5.3 .5)$ in equation (5.2.8).
} 
we may use the near Debye forms for the asymptotic representation of the Bessel functions [36], where in equations (23) below, the superscript "a", denotes the asymptotic form, viz.

and

$$
\left.\begin{array}{c}
\left.J_{m}(x) \sim J_{m}^{(a)}(x)=\left(2 \pi\left(m^{2}-x^{2}\right)^{\frac{1}{2}}\right)^{-\frac{1}{2}} \exp [\pi(m, x)]\right) \\
J_{m}^{\prime}(x) \sim J_{m}^{\prime}(a)(x)=\left(\frac{\left(m^{2}-x^{2}\right)^{\frac{1}{2}}}{2 \pi x}\right)^{\frac{1}{2}} \exp [\pi(m, x)]
\end{array}\right\},
$$

where

$$
\pi(m, x)=\left(m^{2}-x^{2}\right)^{\frac{1}{2}}-m \tanh ^{-1}\left\{\left(1-\frac{x^{2}}{m^{2}}\right)^{\frac{1}{2}}\right\} .
$$

Using equations (23) and (24) in equation (22), the power attenuation coefficient $\alpha$ becomes

$$
\alpha=\frac{W^{2} U^{2}}{\beta d^{2}(I+W) V^{2}} \exp \left\{2 \pi\left(m, k_{2}\left(R_{0}+d\right)\right)\right\}
$$

where we have ignored all terms of order $d / R_{0}$, except in the exponent.

Now, recalling the definition of $\mathrm{m}$, from equation (1), and using a Taylor series expansion of $\pi(m, x)$, of equation (24), in powers of $d / R_{0}$, we find

$$
\pi\left(\beta R_{0}, k_{2}\left(R_{0}+d\right)\right)=R_{0}\left(\frac{W}{k_{2} d}-\beta \tanh ^{-1}\left(\frac{W}{\beta d}\right)\right)+W+O\left(\frac{d}{R_{0}}\right),
$$

where $\mathrm{w}=\mathrm{d}\left(\beta^{2}-\mathrm{k}_{2}^{2}\right)^{\frac{1}{2}}$.

If we wish to retain only the first order correction, i.e. $W$, in equation (26), we require

$$
O\left(\frac{d}{R_{0}}\right) \ll 1
$$

which from equation (24), considering the second order term in the expansion, becomes

$$
\frac{(\beta d)^{2}}{2 W}<\frac{R_{0}}{d}
$$

which can certainly be satisfied for some bending radius $R_{0}$. 
satisfied, we find

$$
\alpha=\frac{U^{2} W^{2}}{\left(\beta d^{2}\right) V^{2}(1+W)} \exp \left\{2 W+2 R_{0}\left(\frac{W}{d}-\beta \tanh ^{-1}\left(\frac{W}{\beta d}\right)\right)\right\}
$$

For weakly guiding dielectric optical waveguides $(W / B d)<1$ and we may use a power series expansion of $\tanh ^{-1}(x)$, for $x<1$ [37], in the exponent of equation (28), so that

$$
2 R_{0}\left(\frac{W}{d}-\beta \tanh ^{-1}\left(\frac{W}{\beta d}\right)\right)=-\frac{2}{3} \frac{W^{3}}{(\beta d)^{2}}\left(\frac{R_{0}}{d}\right)-\frac{2}{5}\left(\frac{W^{5}}{(\beta d)^{4}}\right)\left(\frac{R_{0}}{d}\right) .
$$

If we retain only the first term in $(W / B d)$ in equation (29), we require

$$
\frac{2}{5}\left(\frac{W^{5}}{(B d)^{4}}\right) \frac{R_{0}}{d} \ll 1
$$

which from using

$$
B d \sim \frac{\mathrm{V}}{\theta_{\mathrm{c}}}
$$

where

$$
\mathrm{v}=\mathrm{k}_{1} \rho \theta_{\mathrm{c}}=\mathrm{k}_{1} \rho\left(1-\frac{\varepsilon_{2}}{\varepsilon_{1}}\right)^{\frac{1}{2}}
$$

for weakly guiding waveguides, equation (30), becomes

$$
\frac{R_{0}}{d} \ll \frac{5 v^{4}}{2 w^{5} \theta_{C}^{4}}
$$

Then, using equation (29), retaining only the first order terms, we find that the power attenuation coefficient of the even $\mathrm{TE}$ mode is

$$
\alpha=\frac{U^{2} W^{2}}{\beta d^{2}(1+W) \cdot V^{2}} \exp \left\{2 W-\frac{2 W^{3}}{3(\beta \rho)^{2}}\left(\frac{R_{0}}{\rho}\right)\right\}
$$

which is valid for the range of bending radii satisfying, from equation (27) and (32),

$$
\frac{v^{2}}{2 w \theta_{c}^{2}} \ll \frac{R_{0}}{d} \ll \frac{5 v^{4}}{2 w^{5} \theta_{c}^{4}}
$$

From this discussion, it would also appear to be a great temptation to write the exponent purely as a function of $w, v, \theta_{C}$ and $R_{0} / d$. Rewriting the exponent of equation (33) as 


$$
\frac{W^{3}}{(\beta d)^{2}}\left(\frac{R_{0}}{d}\right)=\frac{W^{3}}{V^{2}} \theta_{C}^{2}\left(\frac{R_{0}}{d}\right)\left(1+\frac{U^{2}}{(\beta d)^{2}}\right) .
$$

Since $U / B d \ll 1$, we can ignore the second term in equation (35) if

$$
\frac{R_{0}}{d}<<\frac{V^{4}}{w^{3} \cdot U^{2} \theta_{C}^{4}}
$$

and then equation (33) becomes

$$
\alpha=\frac{U^{2} W^{2}}{B d^{2}(I+W) V^{2}} \exp \left\{2 W-\frac{2}{3} \frac{W^{3}}{V^{2}} \theta_{C}^{2}\left(\frac{R_{0}}{d}\right)\right\}
$$

which is valid within the restrictions of equation (34) and (36).

From an investigation of equations (34) and (36), modes far from cutoff satisfy

$$
\begin{aligned}
& \frac{\mathrm{W}}{\mathrm{V}} \sim 1 \\
& \frac{\mathrm{U}}{\mathrm{V}} \ll 1
\end{aligned}
$$

so that we find the upper limit of equation (34) is more restrictive than the upper limit for equation (37). Thus, we may write

$$
\alpha=\frac{U^{2} W^{2}}{B d^{2}(I+W) V^{2}} \exp \left\{2 W-\frac{2}{3} \frac{W^{3}}{V^{2}} \theta_{C}^{2}\left(\frac{R_{0}}{d}\right)\right\}
$$

provided

$$
\frac{V^{2}}{2 w \theta_{c}^{2}} \ll \frac{R_{0}}{d} \ll \frac{5 V^{4}}{2 W^{5} \theta_{c}^{4}}
$$




\section{APPENDIX B \\ RAY OPTICAL ANALYSIS OF THE RADIATION LOSS \\ IN A BENT SLAB WAVEGUIDE}

In Appendix A, we used the Surface Current Method to derive the radiative loss from a mode due to a bend in the slab waveguide axis. In this analysis, we have employed various assumptions as to the behaviour of the straight fibre mode in the bend. By application of the generalised Fresnel's law for rays incident on a curved dielectric interface in this appendix, we hope to clarify some of these assumptions.

From the generalised Fresnel's law for rays incident on a concave curved dielectric interface [19] between two homogeneous media, from the more dense medium at an angle $\theta_{i}<\theta_{c}$, where $\theta_{c}$ is the complement of the critical angle, a fraction of the incident energy in the ray is transmitted across the interface and emerges as free radiation in the less dense medium, as in fig. 15. This fraction of the incident power transmitted is $T$, where [19]

$$
T=\frac{4}{\sin \theta_{i}}\left(\sin ^{2} \theta_{c}-\sin ^{2} \theta_{i}\right)^{\frac{1}{2}} \exp \left\{-\frac{2 k_{1} R_{0}}{3 \cos ^{2} \theta_{i}}\left(\sin ^{2} \theta_{c}-\sin ^{2} \theta_{i}\right)^{\frac{3}{2}}\right\}
$$

for rays with $\theta_{i} / \theta_{C} \sim 1$, where

$$
\theta_{C}=\sin ^{-1}\left(1-\frac{\varepsilon_{2}}{\varepsilon_{1}}\right)^{\frac{1}{2}}
$$

and $R_{0}$ is the local radius of curvature of the surface. In a curved waveguide, each time the ray is incident on this surface, it loses a fraction $T$ of its power, thus the power attenuation coefficient of the ray is

$$
\alpha=\mathrm{NT}
$$




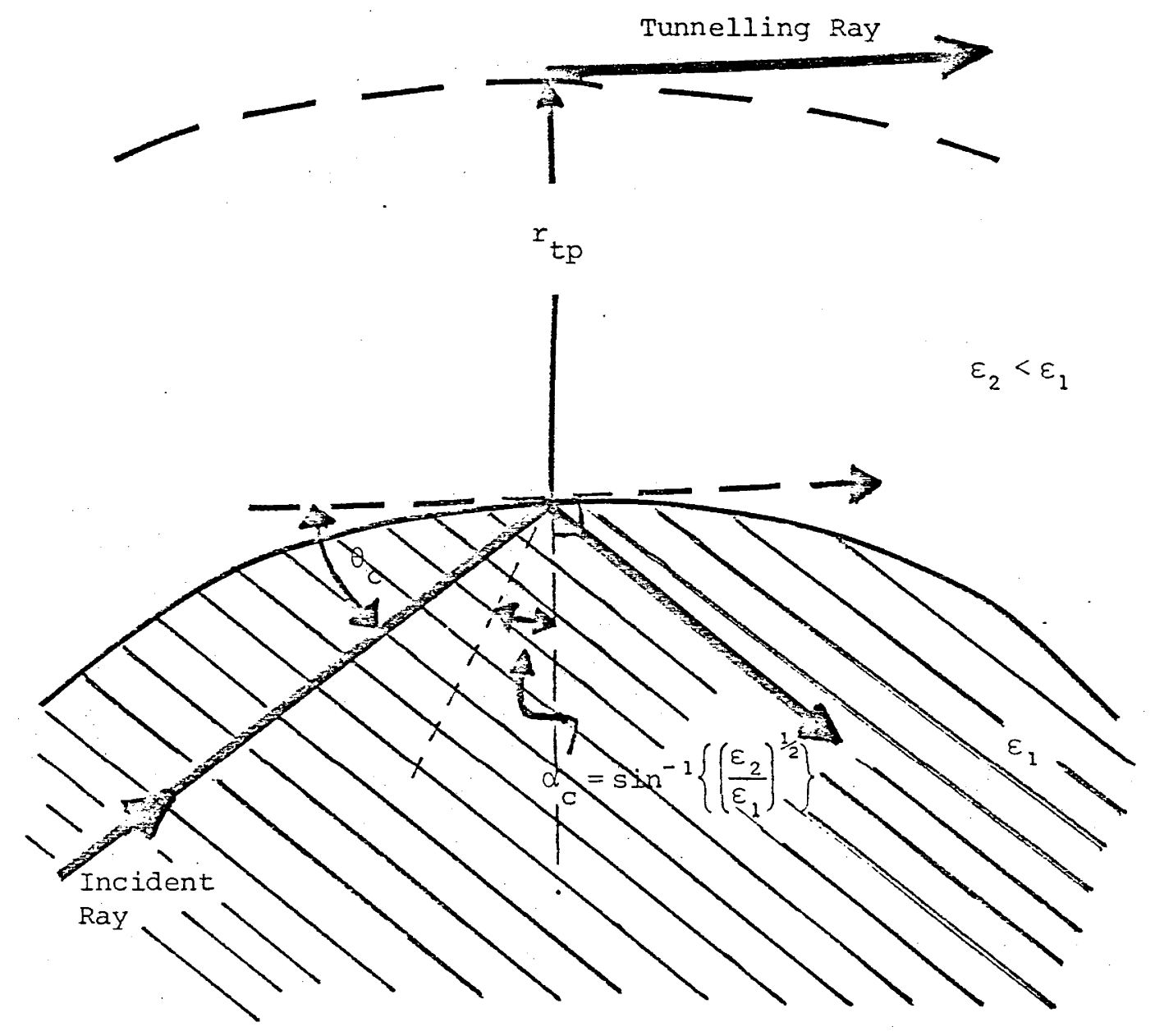

Fig. 15: Ray optical interpretation of the curvature induced radiation loss. The tunnelling leaky ray emerges at a distance, $r$ tp' from the
core-cladding interface. 
where $\mathrm{N}$ is the number of times the ray is incident on this interface per unit length of bend. If we assume that for a slow bend, i.e. $R_{0}>1$, this number of bounces is the same as in the straight slab, then

$$
N=\frac{1}{z_{\text {tot }}}=\frac{1}{2\left(z_{g \circ}+z_{G H}\right)}
$$

where $z_{g o}$ is the geometric optics distance of a ray at angle $\theta_{i}$ to the axis, between successive reflections, and $z_{\mathrm{GH}}$ is the Goos-Hänchen shift of the ray [38] that occurs at the interface at every reflection, as in fig. 16 .

Then, using the well known formulae [38]

$$
\left.\begin{array}{rl}
2 z_{g O} & =\frac{4 d}{\tan \theta_{i}} \\
2 z_{G H} & =\frac{4}{k_{1} \tan \theta_{i}\left(\sin ^{2} \theta_{C}-\sin ^{2} \theta_{i}\right)^{\frac{1}{2}}}
\end{array}\right\}
$$

and using equations (4) and (5) in (3)

$$
\begin{gathered}
\alpha=\frac{\left(\sin ^{2} \theta_{c}-\sin ^{2} \theta_{i}\right) k_{1}}{\cos ^{2} \theta_{i}\left\{1+k_{1} d\left(\sin ^{2} \theta_{c}-\sin ^{2} \theta_{i}\right)^{\frac{1}{2}}\right\}} \\
\times \exp \left\{-\frac{2 k_{1} R_{0}}{\cos ^{2} \theta_{i}}\left(\sin ^{2} \theta_{c}-\sin ^{2} \theta_{i}\right)^{\frac{3}{2}}\right\} .
\end{gathered}
$$

Now consider the two rays, $A$ and $B$, of fig. 4 entering the curved segment of waveguide from the straight segment. In the straight fibre, the two rays are parallel with angle $\theta_{z}$ to the axis. In the bent section, ignoring the scattering of the rays due to the discontinuity, these rays have different angles of incidence with the outer corecladding interface. From elementary geometry,

$$
\left.\begin{array}{l}
\theta_{i}^{A}=\theta_{z} \\
\theta_{i}^{B}=\theta_{z}+\frac{d}{R_{0}} \cot \theta_{z}
\end{array}\right\}
$$

for $d / R_{0} \ll 1$. Although the change in angle is small, the exponential 


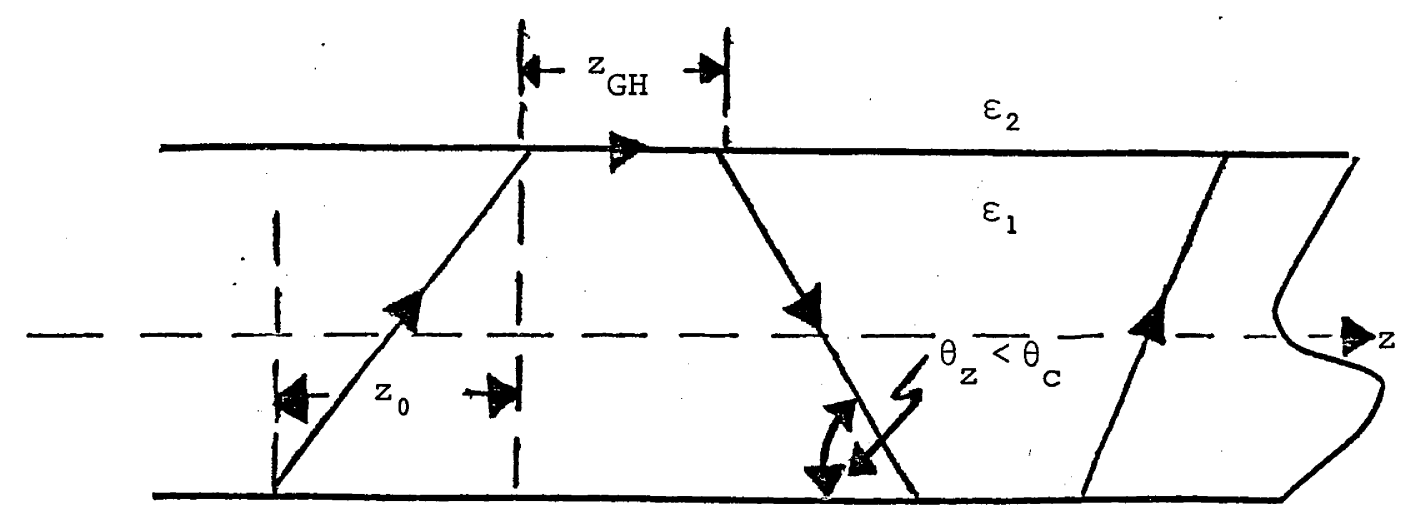

Fig. 16: Propagation of a ray along a slab waveguide. The Goos-Hänchen shift upon reflection $z_{\mathrm{GH}}$ must be included for an accurate description of the propagation of the rays. 
component in equation (6) has a factor proportional to $R_{0}$ so that using a Taylor series expansion of the exponent in powers of $d / R_{0}$, we find

$$
\left.\begin{array}{rl}
\frac{\alpha_{A}}{\alpha_{B}} & =e^{-2 k_{1} \alpha\left(\sin ^{2} \theta_{C}-\sin ^{2} \theta_{i}\right)^{\frac{1}{2}}} \\
& =e^{-2 W}
\end{array}\right\}
$$

and

$$
\alpha_{B}=\frac{w^{2} e^{2 W}}{k_{1} \cdot d^{2}(1+W)} \exp \left\{-\frac{2}{3} \frac{W^{3}}{\left(k_{1} d\right)^{2}} \frac{R_{0}}{d}\right\} \text {, }
$$

where we have used the transformation [39]

$$
\begin{aligned}
& \left.\begin{array}{l}
\mathrm{U}=\mathrm{k}_{1} \mathrm{~d} \sin \theta_{z} \\
\mathrm{v}=\mathrm{k}_{1} \mathrm{~d} \sin \theta_{\mathrm{C}}
\end{array}\right\} \\
& W=\left(V^{2}-U^{2}\right)^{\frac{1}{2}}=\left(k_{1} d\right)\left(\sin ^{2} \theta_{C}-\sin ^{2} \theta_{i}\right)^{\frac{1}{2}} .
\end{aligned}
$$

Now, from a comparison of equation (9) with equation (A.33) for $(U / V) \sim 1$, which corresponds to the regime of validity of equation (1) for $T$, (from equations (10)), we find

$$
\alpha_{B}^{\text {ray }}=\alpha_{\text {mode }}
$$

Thus, we can see that the ray optics analysis agrees with the modal attenuation if we choose to characterise the family of rays that make up the mode by the ray that makes an angle $\theta_{z}$ to the axis in the bent waveguide. 


\section{APPENDIX C \\ RADIATION FROM A BEND IN AN OPTICAL FIBRE OF CIRCULAR CROSS SECTION}

In this appendix, we calculate the radiative loss from a mode of the straight optical fibre of circular cross section due to a planar bend in the axis of the fibre. As for the analysis of the slab waveguide, we consider a complete torus, as in fig. $2 \mathrm{~b}$, so that we exclude all contributions due to changes in the radius of curvature of the fibre axis. In the final calculations for the total radiated power, we will differentiate between two classes of modes, i.e. the low order modes, $\ell \sim 1$, and the higher order modes, $\ell \gg 1$. This differentiation between the two mode sets allows for a greater understanding of the bending loss problem from a geometric optics interpretation of the loss process for the higher order modes. We will discuss this in section 4 .

In general, the position vector of the core-cladding interface over which the Surface Current Integral, equation (5.2.12), will be applied is defined by

$$
\stackrel{\mathrm{R}}{\sim}^{\prime}=\underset{\sim}{\mathrm{R}}+\underline{\sim}^{\prime},
$$

where $\mathrm{R}_{0}$ is the position vector of the fibre axis and ${\underset{\sim}{\sim}}^{\prime}$ is the position vector of the surface relative to the fibre axis.

Defining the local cylindrical co-ordinate system about the fibre axis $r^{\prime}, X_{,} z^{\prime}$ with associated local cartesian co-ordinate system $x^{\prime}, y^{\prime}, z^{\prime}$, as in fig. 5, and the spherical polar co-ordinate system $R$, $\theta, \phi$ relative to the centre of curvature of the waveguide axis, with a radius of a curvature $R_{0}$, as in fig. 5 , we find

$$
\stackrel{R}{\sim}_{0}=R_{0}\left(\cos \phi^{\prime} \hat{\underline{x}}+\sin \phi^{\prime} \hat{\underline{Z}}\right)
$$

and 


$$
\underline{r}^{\prime}=\rho \cos \chi{\underset{\sim}{\hat{x}}}^{\prime}+\sin \chi{\underset{\sim}{\hat{y}}}^{\prime},
$$

where in terms of the global cartesian co-ordinates $x, y, z$ about the centre of curvature 0 , we have

$$
\left.\begin{array}{l}
\hat{\sim}^{\prime}=\hat{\sim} \\
\hat{\underline{y}}^{\prime}=-\left(\cos \phi^{\prime} \underset{\sim}{\hat{x}}+\sin \phi^{\prime} \underset{\sim}{\hat{y}}\right) \\
\hat{\sim}^{\prime}=-\sin \phi^{\prime} \underset{\sim}{\hat{x}}+\cos \phi^{\prime} \underset{\sim}{\hat{y}}
\end{array}\right\}
$$

so that equation ( 1 ) becomes

$$
\stackrel{R}{\sim}^{\prime}=\left(R_{0}-\rho \sin \chi\right) \cos \phi^{\prime} \underset{\sim}{\hat{x}}+\left(R_{0}-\rho \sin \chi\right) \sin \phi^{\prime} \underset{\sim}{\hat{y}}+\rho \cos \chi \underset{\sim}{\hat{z}} \cdot
$$

Now from the symmetry of the complete toroid, we need only calculate the radiation field at one point, viz. for $\phi=\pi / 2$ and for $\phi=\pi / 2$

$$
\underset{\sim}{\hat{R}}=\sin \theta \underline{\underline{z}}+\cos \theta \underset{\sim}{\hat{z}}
$$

and

$$
\stackrel{\hat{\mathrm{R}}}{\sim} \mathbb{\sim}^{\prime}=\left(\mathrm{R}_{0}-\rho \sin \chi\right) \sin \theta \sin \theta^{\prime}+\rho \cos \chi \cos \theta .
$$

From Appendix 5.B, we need only calculate one vector integral for the azimuthal travelling wave formulation of the fibre modes, i.e.

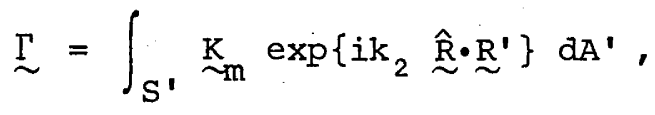

where we now have $S^{\prime}$ as the core-cladding interface

$$
\underset{\mathrm{m}}{\mathrm{K}}=\underset{\sim}{\hat{\mathrm{n}}} \times \underset{\sim}{\mathrm{E}},
$$

where now, $\hat{\sim}=\hat{\underline{x}}^{\prime}$, the local radial vector in cylindrical co-ordinates, so that

$$
\underset{\sim m}{\mathrm{~K}}=-\mathrm{E}_{z} \hat{\chi}+\mathrm{E}_{x} \hat{z}^{\prime}
$$

where $\underset{\sim}{\hat{x}}, \hat{z}^{\prime}$ are the unit vectors of the local cylindrical system

$$
\underset{\sim}{\hat{x}}=-\sin \chi \hat{x}^{\prime}+\cos \chi \underline{\sim}^{\hat{y}^{\prime}} .
$$

Using equations (3) together with (9) in equation (8), we have 


$$
\begin{aligned}
\underset{\sim}{\mathrm{K}}= & \left\{E_{z^{\prime}} \cos \chi \cos \phi^{\prime}-E_{\chi} \sin \phi^{\prime}\right\} \underset{\sim}{\hat{x}} \\
& +\left\{E_{z^{\prime}}, \cos \chi \sin \phi^{\prime}+E_{X} \cos \phi^{\prime}\right\} \underset{\sim}{\hat{z}}+E_{z^{\prime}} \sin \chi \underset{\sim}{\hat{z}} .
\end{aligned}
$$

From equations $(2.2 .43)-(2.2 .45)$, the electric fields evaluated at the core-cladding interface are

$$
\left.\begin{array}{l}
E_{z}^{\prime}=-\frac{|\ell|}{\ell} A_{\ell \mp 1} B_{\ell \mp 1} e^{-i \ell \chi-i m \phi^{\prime}} \\
E_{X}=A_{\ell \mp 1} e^{-i \ell \chi-i m \phi^{\prime}}
\end{array}\right\},
$$

where $A_{\text {lFl }}$ is the power normalisation coefficient, chosen to ensure, unit power is propagating in the incident mode, defined in equation (2.2.53), and $B_{\ell \mp 1}$ is the magnitude of the longitudinal electric field at the core-cladding interface

$$
B_{\ell \mp 1}=\frac{i W}{k_{2} \rho} \cdot\left(\frac{H_{l}^{(1)}(i W)}{H_{l \mp 1}^{(1)}(i W)}\right) \text {. }
$$

where we have already employed the phase matching along the axis, via

$$
\beta d z=m d \phi=\beta R_{0} d z
$$

which provides

$$
m=B R_{0} \cdot
$$

Using equations (11) in (10) we have

$$
\begin{aligned}
\stackrel{\sim m}{m}^{\mathrm{K}}= & { }^{A_{\ell \mp 1}} \mathrm{e}^{-i \ell X-i m \phi^{\prime}}\left(\underset{\sim}{\hat{x}}\left\{-\frac{|\ell|}{\ell} \mathrm{B}_{\ell \mp 1} \cos X \cos \phi^{\prime}-\sin \phi^{\prime}\right\}\right. \\
& \left.+\hat{\sim}\left\{-\frac{|\ell|}{\ell} B_{\ell \mp 1} \cos X \sin \phi^{\prime}+\cos \phi^{\prime}\right\}+\hat{z}\left\{-\frac{|\ell|}{\ell} \sin X B_{\ell \mp 1}\right\}\right)^{\prime}
\end{aligned}
$$

where the $|\ell| / \ell$ terms are included for the $\pm \ell$ modes and subscripts on

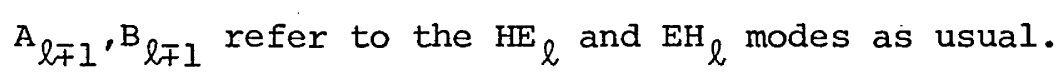

In Appendix D, we demonstrate the equivalence of the calculations of the $\pm l$ modes, so that we need only calculate equation (13) for one " $\ell$ " mode. We choose $\ell>0$ so that equation (13) now becomes 


$$
\begin{aligned}
& {\underset{\sim m}{\mathrm{~m}}}^{\mathrm{A}}=\mathrm{A}_{\ell \mp 1} \mathrm{e}^{-i \ell X-i m \phi^{\prime}}\left\{\underset{\hat{x}}{\hat{x}}\left(-\mathrm{B}_{\ell \mp 1} \cos X \cos \phi^{\prime}-\sin \phi^{\prime}\right)\right. \\
& +\underset{\sim}{\hat{y}}\left(-B_{\ell \mp 1} \cos \chi \sin \phi^{\prime}+\cos \phi^{\prime}\right)-\underbrace{\hat{z}}_{\ell \neq 1} \cdot B_{\ell \mp 1} \sin \chi\}
\end{aligned}
$$

and using equation (14) in equation (7) together with equation (6), we find

$$
\begin{aligned}
& \Sigma=\rho A_{\ell \mp 1} \int_{0}^{2 \pi} d x\left(R_{0}-\rho \sin \chi\right) \int_{0}^{2 \pi} d \phi^{\prime} \\
& \text { - }\left\{\left(\exp \left\{i k_{2} \rho \cos \theta \cos \chi+i k_{2}\left(R_{0}-\rho \sin \chi\right) \sin \theta \sin \phi^{\prime}-i m \phi^{\prime}-i \ell \chi\right\}\right)\right. \\
& \text { - }\left(-\underset{\sim}{\hat{x}}\left(B_{\ell \mp 1} \cos X \cos \phi^{\prime}+\sin \phi^{\prime}\right)+\hat{z}\left(-B_{\ell \mp 1} \cos X \sin \phi^{\prime}+\cos \phi^{\prime}\right)\right. \\
& \left.\left.-\hat{z}\left(B_{\ell \mp 1} \sin x\right)\right)\right\} \text {. }
\end{aligned}
$$

and from equation (5.2.12), we find

$$
S^{R}=\left(\frac{\varepsilon_{2}}{\mu}\right)^{\frac{1}{2}} \frac{k_{2}^{2}}{16(\pi R)^{2}}|\underset{\sim}{\Gamma} \cdot(\underset{\sim}{\hat{x}} \pm i(\underset{\sim}{\hat{y}} \cos \theta-\underset{\sim}{\hat{z}} \sin \theta))|^{2},
$$

where the + and - signs in equation (16) refer to the $\mathrm{HE}$ and $\mathrm{EH}$ mode respectively.

From an investigation of equation (15), we can observe that the integral over $\phi^{\prime}$ is in the form of integral representations of Bessel functions of the first kind [36], i.e.

$$
J_{n}(x)=\frac{1}{2 \pi} \int_{0}^{2 \pi} \exp \{i x \sin \theta-i n \theta\} d \theta
$$

and using the recurrence relations of Bessel functions [36], we find

$$
\begin{aligned}
& \Gamma=2 \pi \rho A_{\ell \mp 1} \int_{0}^{2 \pi} d x\left\{\exp \left\{i k_{2} \rho \cos \theta \cos \chi-i \ell \chi\right\}\right. \\
& \text { - }\left(-\hat{\hat{x}}\left(B_{\ell \mp I} \frac{\mathrm{m}}{\mathrm{k}_{2} \sin \theta} \mathrm{J}_{\mathrm{m}}(Y) \cos X-i J_{m}^{\prime}(Y)\left(R_{0}-\rho \sin X\right)\right)\right. \\
& +\hat{\underline{y}}\left(-i B_{\ell \mp 1} J_{m}^{\prime}(Y) \cos X\left(R_{0}-\rho \sin X\right)+\frac{m}{k_{2} \sin \theta} J_{m}(Y)\right) \\
& \left.\left.-\hat{z}\left\{B_{\ell \mp I} J_{m}(Y) \sin X\left(R_{0}-\rho \sin X\right)\right)\right]\right\} \text {, }
\end{aligned}
$$


where the arguments of the Bessel functions is $Y$, where

$$
Y=k_{2}\left(R_{0}-\rho \sin \chi\right) \sin \theta \text {. }
$$

The ' indicates differentiation with respect to the argument $\mathrm{Y}$.

The remaining integral of equation (17) over $X$, cannot be evaluated in a closed analytic form. However, since the incident modal field is a bound mode of the straight fibre

$$
\beta>\mathrm{k}_{2}
$$

and from equation (12) this means that the argument of the Bessel functions is less than the order. Thus we can use the near Debye asymptotic forms of the Bessel functions [36]. We write the asymptotic forms as $J_{m}^{(a)}(Y)$ and $J_{m}{ }^{(a)}(Y)$ where the superscript denotes the asymptotic nature of the expression. Thus

and

$$
\left.\begin{array}{rl}
J_{m}(Y) \sim J_{m}^{(a)}(Y) & =\left(2 \pi\left(m^{2}-Y^{2}\right)^{\frac{1}{2}}\right)^{-\frac{1}{2}} \exp \{\pi(m, Y)\} \\
J_{m}^{\prime}(Y) \sim \underset{m}{J_{m}(a)}(Y) & =\left(\frac{\left(m^{2}-Y^{2}\right)}{2 \pi Y}\right)^{\frac{1}{2}} \exp \{\pi(m, Y)\}
\end{array}\right\}
$$

where

$$
\pi(m, Y)=\left\{\left(m^{2}-Y^{2}\right)-m \tanh ^{-1}\left(\left[1-\frac{m^{2}}{Y^{2}}\right)^{\frac{1}{2}}\right)\right\}
$$

Since all integrals over $X$ in equation (17) can be written as below, we confine our discussion to this integral

$$
\Psi(Y)=\int_{0}^{2 \pi}\left\{\begin{array}{c}
J_{m}(Y) \\
J_{m}^{\prime}(Y)
\end{array}\right\} \exp \left\{-i p X+i k_{2} \rho \cos \theta \cos X\right\}
$$

where $\mathrm{p}$ is some integer. Using the asymptotic expressions for the Bessel functions, as in (18), we observe that the integral of equation (20) has an extra term in the exponent due to the exponent of the asymptotic expression for the Bessel function. In order to isolate the $X$ dependence in this exponential term, we use the fact that

$$
\frac{\rho}{R_{0}} \ll 1
$$


and use a Taylor series expansion of $\pi(m, Y)$ about $\rho / R_{0}=0$, so that defining

$$
\mathrm{Y}_{0}=\mathrm{k}_{2} \mathrm{R}_{0} \sin \theta
$$

we find

$$
\pi(m, Y)=\pi\left(m, Y_{0}\right)-k_{2} \rho \sin \theta \sin X+o\left(\frac{\rho}{R_{0}}\right) .
$$

If we wish to retain only the first order correction, viz. $\left(k_{2} \rho \sin \theta \sin X\right)$, we require

$$
O\left(\frac{\rho}{R_{0}}\right) \ll I
$$

which from evaluation of the next term in the Taylor series expansion requires

$$
\frac{(\beta \rho)^{2}}{2 \mathrm{R}_{0}\left(\beta^{2}-\mathrm{k}_{2}^{2} \sin ^{2} \theta\right)^{\frac{1}{2}}} \ll 1 \text {. }
$$

Using equation (22), ignoring higher order terms in $\rho / R_{0}$, then, we find equation (20) can be written as

$$
(Y) \sim\left\{\begin{array}{c}
J_{m}^{(a)}\left(Y_{0}\right) \\
J_{m}^{\prime}(a) \\
\left(Y_{0}\right)
\end{array}\right\} \int_{0}^{2 \pi} d X\{\exp \{W \sin (X-i \delta)-i p X\}\},
$$

where

and

$$
\left.\begin{array}{rl}
W & =\rho\left(\beta^{2}-k_{2}^{2}\right)^{\frac{1}{2}} \\
\sinh \delta & =\frac{k_{2} \rho \cos \theta}{W}
\end{array}\right\} \text {. }
$$

After changing variables, equation (24) is recognised as the integral representation of the modified Bessel function $I_{p}(W)$ [36], and thus equation (24) becomes

$$
\psi\left(Y_{0}\right)=2 \pi(-i)^{p}\left\{\begin{array}{c}
J_{m}^{(a)}\left(Y_{0}\right) \\
J_{m}^{\prime}(a) \\
\left(Y_{0}\right)
\end{array}\right\} e^{p \delta} I_{p}(W)
$$

and this is a valid approximation to equation (20) provided equation (23) is satisfied. 
Using equation (20) together with its solution equation (26) in equation (17) for $\underset{\sim}{\Gamma}$ we find

$$
\begin{aligned}
& I=(-i)^{\ell-1} e^{\ell \delta}\left(2 \pi^{2} \rho R_{0}\right) J_{m}^{(a)}\left(Y_{0}\right) A_{\ell \mp 1} \\
& \times\left\{\hat{x}\left(B_{\ell \mp 1} \frac{\beta}{k_{2} \sin \theta}\left(I_{\ell-1}-I_{\ell+1} e^{-2 \delta}\right) e^{\delta}+\frac{2\left(\beta^{2}-k_{2}^{2} \sin ^{2} \theta\right)^{\frac{1}{2}}}{k_{2} \sin \theta} I_{\ell}\right)\right. \\
& -i \underset{\sim}{\hat{y}}\left(B_{\ell \mp 1} \frac{\left(B^{2}-k_{2}^{2} \sin ^{2} \theta\right)^{\frac{1}{2}}}{k_{2} \sin \theta}\left(I_{\ell-1}-I_{\ell+1} e^{-2 \delta}\right) e^{\delta}+2 I_{\ell}\right) \\
& \left.+i \underline{z}\left(B_{\ell \mp 1}\left(I_{\ell-I}+I_{\ell+1} e^{-2 \delta}\right) e^{\delta}\right)\right\} \text {, }
\end{aligned}
$$

where we have ignored all non-exponent terms of order $\rho / R_{0}$. The argument of the modified Bessel function is $\mathrm{W}$ defined in equation (25).

Rewriting equation (20) as

$$
\Gamma=e^{\pi\left(m, Y_{0}\right)} \stackrel{\Lambda}{\sim}
$$

where

$$
\begin{aligned}
& \Lambda=(-i)^{l-1}\left(2 \pi^{2} \rho R_{0}\right)\left(2 \pi\left(m^{2}-Y_{0}^{2}\right)^{\frac{1}{2}}\right)^{-\frac{1}{2}} A_{l \mp 1} e^{\ell \delta} \\
& \times\left\{\hat{\underline{x}}\left(B_{\ell \mp 1} \frac{B}{k_{2} \sin \theta}\left(I_{\ell-1} e^{\delta}-I_{\ell+1} e^{-\delta}\right)+\frac{2\left(\beta^{2}-k_{2}^{2} \sin ^{2} \theta\right)^{\frac{1}{2}}}{k_{2} \sin \theta} I_{\ell}\right)\right. \\
& -i \hat{z}\left(B_{\ell \mp 1} \frac{\left(B^{2}-k^{2} \sin ^{2} \theta\right)^{\frac{1}{2}}}{k_{2} \sin \theta}\left(I_{\ell-1} e^{\delta}-I_{\ell+1} e^{-\delta}\right)+2 I_{\ell}\right) \\
& \left.+i \underset{\sim}{\hat{z}}\left\{B_{\ell \mp 1}\left(I_{\ell-1} e^{\delta}+I_{\ell+1} e^{-\delta}\right)\right\}\right\}
\end{aligned}
$$

we find, from equations (28): and (29) in (16),

$$
S^{R}=\left(\frac{\varepsilon_{2}}{\mu}\right)^{\frac{1}{2}} \frac{k_{2}^{2}}{16(\pi R)^{2}} e^{2 \pi\left(m, Y_{0}\right)} \mid \Lambda \cdot\left(\underset{\sim}{\hat{x}} \pm\left. i(\underset{\sim}{\hat{y}} \cos \theta-\underset{\sim}{\hat{z}} \sin \theta)\right|^{2}\right.
$$

and

$$
P^{R}=2 \pi R^{2} \int_{0}^{\pi} S^{R} \sin \theta d \theta
$$

The form of equations (30) suggests that the evaluation of equation (31) is most easily accomplished by an asymptotic evaluation 
using Laplace's method [40]. This technique basically uses the fact that strongly exponentially dependent integrals can be approximated by Gaussian distributions about their point of maximum amplitude, i.e.

$$
\int_{a}^{b} e^{\lambda h(x)} g(x) d x \sim\left(-\frac{2 \pi}{\lambda h^{\prime \prime}\left(x_{0}\right)}\right)^{\frac{1}{2}} e^{\lambda h\left(x_{0}\right)} g\left(x_{0}\right)
$$

for $\lambda>>1$, where $x_{0}$ is the point that corresponds to the maximum contribution of the integral; $x_{0}$ satisfies

$$
\frac{d}{d x}\left\{h\left(x_{0}\right)\right\}=0
$$

Using this technique for the evaluation of equation (31) using $S^{R}$ defined in equation (30), we find that for low azimithal mode numbers the exponent entering into equation (31) is $\pi\left(m, Y_{0}\right)$, so that the point of maximum contribution in the $\theta$ direction corresponds to

$$
\frac{d}{d \theta}\left\{\pi\left(m, Y_{0}\right)\right\}=0
$$

which from equation (19) for $\pi\left(m, Y_{0}^{\prime}\right)$ using equation (21) for $Y_{0}$, is satisfied by

$$
\theta_{0}=\frac{\pi}{2}
$$

For higher order modes, $\ell>>1$, there is an additional exponential term due to the $\exp \{2 \delta l\}$ with $\delta$ defined in equation (25) so that the angle $\theta$ with maximum contribution to the radiation field is the solution of

$$
\frac{d}{d \theta}\left\{\pi\left(m, Y_{0}\right)+l \delta\right\}=0
$$

which for $\rho / R_{0}<1$ is satisfied by

where

$$
\theta=\frac{\pi}{2}-\psi
$$

$$
\psi=\frac{k_{2} l \rho^{2}}{R_{0} W^{2}} \ll 1 \text {, }
$$

i.e. for higher order modes the radiation does not lie in the plane of 
the bend as for the low order modes $(\theta=\pi / 2$ is the plane of the bend as in fig. 5b) but is slightly out of the plane due to the skewness of the modal ray family.

The accuracy of the Laplace method can be checked by determining the spread of the function about the position of maximum contribution. This spread can be considered as the half width of the Gaussian approximation. Thus if

and

$$
\left.\begin{array}{c}
\lambda h^{\prime \prime}\left(\mathrm{x}_{0}\right)<0 \\
\left|\lambda \mathrm{h}^{\prime \prime}\left(\mathrm{x}_{0}\right)\right|>1
\end{array}\right\}
$$

the approximation is valid [40]. From equation (35) and (37), to leading order, the half width of the high and low order modes is the same and

$$
\frac{\mathrm{d}^{2}}{\mathrm{~d} \theta^{2}}\left\{\pi\left(\mathrm{m}, \mathrm{Y}_{0}\right)+l \delta\right\} \sim-\left.\frac{2 \mathrm{R}_{0} \mathrm{~W}}{\rho}\right|_{\theta=\theta_{0}} .
$$

For $R_{0} / \rho \gg 1$, then the Laplace method asymptotic evaluation of the integral for the total power radiated, $\mathrm{P}^{\mathrm{R}}$, should be extremely accurate.

$$
\text { Thus, using equations (34) - (40) in equation (31) together }
$$

with equation (30), the asymptotic evaluation of the total power radiated becomes

$$
\begin{aligned}
P^{R}= & \left(\frac{\varepsilon_{2}}{\mu}\right)^{\frac{1}{2}}\left(\frac{\pi \rho}{W R_{0}}\right)^{\frac{1}{2}} \frac{k_{2}^{2} \rho^{3} \pi^{2} R_{0}}{W}\left(A_{\ell \mp I}\right) \\
& \times\left( \pm B_{\ell \mp I} I_{\ell \mp I}(W)+\frac{W}{k_{2} \rho} I_{\ell}(W)\right)^{2} \exp \left\{2 \pi\left(\beta R_{0}, k_{2} R_{0} \cos \psi\right)+2 \ell \delta\right\},
\end{aligned}
$$

where

and

$$
\left.\begin{array}{ll}
\psi=0 & \text { for } \ell \sim 1 \\
\psi=\frac{\ell k_{2} \rho^{2}}{R_{0} W^{2}} & \text { for } l>>1
\end{array}\right\}
$$

$$
\left.\begin{array}{rl}
\delta & =\operatorname{arcsinh}\left\{\frac{k_{2} \rho \sin \psi}{w}\right\} \\
& \sim \frac{k_{2} \rho \psi}{w}
\end{array}\right\} .
$$

Since the incident mode was normalised to unit guided power, the power 
attenuation coefficient of the mode, $\alpha$, is

$$
\alpha=\frac{P^{R}}{2 \pi R_{0}}
$$

so that for low order modes $l \sim 1$

$$
\begin{aligned}
\alpha=\frac{\pi}{2}\left(\frac{\varepsilon_{2}}{\mu}\right)^{\frac{1}{2}}\left(\frac{\pi \rho}{W R_{0}}\right)^{\frac{1}{2}} \frac{k_{2}^{2} \rho^{3}}{W}\left(A_{\ell \mp 1}\right)^{2}\left( \pm B_{l \mp I} I_{\ell \mp I}(W)+\frac{W}{k_{2} \rho} I_{\ell}(W)\right)^{2} \\
\times \exp \left\{\frac{2 R_{0}}{\rho}\left(W-\beta \rho \tanh ^{-1}\left(\frac{W}{\beta \rho}\right)\right)\right\}
\end{aligned}
$$

and for higher order modes $\ell>>1$, expanding the exponent $\pi\left(\beta R_{0}, k_{2} R_{0} \cos \psi\right)$ about $\psi=0$, we have

$$
\begin{aligned}
\alpha=\frac{\pi}{2}\left(\frac{\varepsilon_{2}}{\mu}\right)^{\frac{1}{2}}\left(\frac{\pi \rho}{W R_{0}}\right)^{\frac{1}{2}} & \frac{k_{2}^{2} \rho^{3}}{W}\left(A_{\ell \mp 1}\right)^{2}\left( \pm B_{\ell \mp 1} I_{\ell \mp 1}(W)+\frac{W}{k_{2} \rho} I_{\ell}(W)\right) \\
& \times \exp \left\{\frac{2 R_{0}}{\rho}\left(W-\beta \rho \tanh ^{-1}\left(\frac{W}{\beta \rho}\right)\right)+\frac{\ell^{2} k_{2}^{2} \rho^{3}}{W^{3} R_{0}}\right\} .
\end{aligned}
$$

For the weakly guiding dielectric optical fibres, from equation (1la)

$$
B_{\ell \mp 1}=\frac{i W}{k_{2} \rho} \frac{H_{\ell}^{(1)}(i W)}{H_{\ell \mp I}^{(1)}(i W)}
$$

and changing from Hankel functions to modified Bessel functions via [36]

$$
H_{\ell}^{(1)}(i W)=\frac{2}{i \pi}(i)^{-\frac{1}{2} \ell \pi i} \cdot K_{\ell}(W)
$$

so that

$$
\frac{i W}{k_{2} \rho} \frac{H_{\ell}^{(1)}(i W)}{H_{\ell \mp 1}^{(I)}(i W)}=i\left(i^{\mp 1}\right) \frac{w}{k_{2} \rho} \frac{k_{\ell}(W)}{k_{\ell \mp 1}(W)} .
$$

Thus, using the Wronksian relations [36] for Bessel functions of the second kind, we have for equation (46)

and

$$
\begin{aligned}
\alpha_{\ell}= & \frac{\pi}{2}\left(\frac{\varepsilon_{2}}{\mu}\right)^{\frac{1}{2}}\left(\frac{\pi \rho}{W R_{0}}\right)^{\frac{1}{2}} \frac{\rho\left(A_{\ell \mp 1}\right)^{2}}{W\left(K_{\ell \mp 1}(W)\right)^{2}} \\
& \times \exp \left\{\frac{2 R_{0}}{\rho}\left(W-\beta \rho \tanh ^{-1}\left(\frac{W}{\beta \rho}\right)\right) \quad \text { for } \ell \sim 1\right.
\end{aligned}
$$




$$
\alpha=\alpha_{\ell} e^{l^{2} \cdot k_{2}^{2} \rho^{3} / w^{3} R_{0}} \quad \text { for } \ell>1
$$

where $\alpha_{\ell}$ is defined above in equation (49), and for step-index fibres from equation $(2.3 .16), A_{\ell \mp I}$ is

$$
\left(A_{\ell \mp 1}\right)^{-2}=\left(\frac{\varepsilon_{2}}{\mu}\right)^{\frac{1}{2}} \pi \rho^{2}\left(\frac{V}{U}\right)^{2} \frac{H_{l \mp 2}^{(I)}(i W) H_{\ell}^{(1)}(i W)}{\left(H_{\ell \mp 1}^{(I)}(i W)\right)^{2}}
$$

which from above, can be written as

$$
\left(A_{\ell \mp 1}\right)^{-2}=\left(\frac{\varepsilon_{2}}{\mu}\right)^{\frac{1}{2}} \pi \rho^{2}\left(\frac{V}{U}\right)^{2}\left(\frac{K_{\ell \mp 2}(W) K_{\ell}(W)}{\left(K_{\ell \mp 1}(W)\right)^{2}}\right)
$$

and using equations (50)-(51) in equations (48), the power attenuation coefficients for the modes of the step-index fibre become

$$
\text { for } \begin{aligned}
& \ell: \quad \alpha=\frac{1}{2 \rho}\left(\frac{\pi \rho}{W R_{0}}\right)^{\frac{1}{2}} \frac{U^{2}\left[\mathrm{~K}_{\ell \mp 2}(W) \mathrm{K}_{\ell}(W)\right]^{-1}}{W V^{2}} \\
& \times \exp \left\{\frac{2 \mathrm{R}_{0}}{\rho}\left(W-\beta \rho \tanh ^{-1}\left(\frac{W}{\beta \rho}\right)\right)\right\}
\end{aligned}
$$

whereas

$$
\text { for } \ell>>1: \quad \begin{aligned}
\alpha=\frac{1}{2 \rho}\left(\frac{\pi \rho}{W R_{0}}\right)^{\frac{1}{2}} & \frac{U^{2}\left[K_{\ell \mp 2}(W) K_{\ell}(W)\right]^{-1}}{W V^{2}} \\
& \times \exp \left\{\frac{2 R_{0}}{\rho}\left(w-\beta \rho \tanh ^{-1}\left(\frac{W}{\beta \rho}\right)\right)+\frac{\ell^{2} k_{2}^{2} \rho^{3}}{W^{3} R_{0}}\right\} .
\end{aligned}
$$




\section{APPENDIX D \\ EQUIVALENCE OF THE RADIATED POWER FROM THE \\ AZIMUTHAL TRAVELLING WAVE AND STANDING \\ WAVE MODES DUE TO A PLANAR BEND}

In this appendix, we demonstrate that the use of the simplified form of the Surface Current Method for the radiation from a bend in an optical fibre of circular cross section obtained by considering the azimuthal travelling wave modes, is adequate for the determination of the radiation loss of the physically realistic standing wave modes for a planar bend in the fibre axis.

From equation (C.13) we have, for the pseudo surface magnetic current density $\underset{\sim}{\mathrm{K}}$

$$
\begin{aligned}
{\underset{\sim}{\mathrm{K}}}_{\mathrm{m}}= & { }_{\ell \mp I} \mathrm{e}^{-i \ell X-i m \phi^{\prime}}\left\{\underset{\sim}{\hat{\mathrm{x}}}\left\{-\frac{|\ell|}{\ell} \mathrm{B}_{\ell \mp I} \cos X \cos \phi^{\prime}-\sin \phi^{\prime}\right\}\right. \\
& +\underset{\sim}{\hat{\mathrm{y}}}\left\{-\frac{|\ell|}{\ell} \mathrm{B}_{\ell \mp I} \cos X \sin \phi^{\prime}+\cos \phi^{\prime}\right\}-\hat{z}\left\{\frac{|\ell|}{\ell} \sin X \mathrm{~B}_{\ell \mp 1}\right\},
\end{aligned}
$$

where the $|\ell| / \ell$ refers to the $\pm \ell$ modes and the subscripts $l \pm 1$ on $A_{\ell \mp 1}$ and $\mathrm{B}_{\ell \mp I}$ refer to the $\mathrm{HE}_{\ell}$ and $\mathrm{EH}_{\ell}$ modes as usual.

Now, from equation (C.15), we have

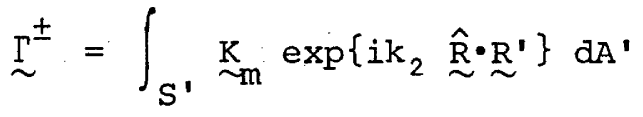

$$
\begin{aligned}
& =\rho A_{\ell \mp 1} \int_{0}^{2 \pi} d \chi\left(R_{0}-\rho \sin X\right) \int_{0}^{2 \pi} d \phi^{\prime} \\
& \times\left\{\exp \left\{i k_{2} \rho \cos \theta \cos \chi+i k_{2}\left(R_{0}-\rho \sin \chi\right) \sin \theta \sin \phi^{\prime}-i m \phi^{\prime}-i \ell \chi\right\}\right. \\
& \times\left\{\hat{x}\left\{-\frac{|\ell|}{\ell} B_{\ell \mp 1} \cos X \cos \phi^{\prime}-\sin \phi^{\prime}\right\}\right.
\end{aligned}
$$




$$
\left.\left.+\hat{\underline{y}}\left\{-\frac{|\ell|}{\ell} \mathrm{B}_{\ell \mp 1} \cos X \sin \phi^{\prime}+\cos \phi^{\prime}\right\}-\hat{\underline{z}}\left\{\frac{|\ell|}{\ell} \sin X \mathrm{~B}_{\ell \mp 1}\right\}\right)\right\}
$$

from which, for the time average radiant energy flux, we require to determine two integrals

$$
\Phi^{ \pm}=\left|\Gamma_{\sim}^{ \pm} \cdot(\underset{\sim}{\hat{x}} \pm i(\underset{\sim}{\hat{y}} \cos \theta-\underset{\sim}{\hat{z}} \sin \theta))\right| \text {. }
$$

Changing variables for $\Gamma^{+}$to $\theta^{+}=\pi / 2-\theta$ and for $\Gamma_{\sim}^{-}$changing to $X^{\prime}=\pi-X$ and $\theta^{-}=\pi / 2+\theta$ such that

$$
\begin{aligned}
\stackrel{\Gamma}{\sim}^{+}= & \rho A_{\ell \mp 1} \int_{0}^{2 \pi} \mathrm{d} \chi\left(R_{0}-\rho \sin \chi\right) \int_{0}^{2 \pi} \mathrm{d} \phi^{\prime} \\
& \times\left\{\exp \left\{+i k_{2} \rho \sin \theta^{+} \cos \chi+i k_{2}\left(R_{0}-\rho \sin \chi\right) \cos \theta^{+} \sin \phi^{\prime}-i m \phi^{\prime}-i \ell \chi\right\}\right. \\
& \times\left(\hat{x}\left(-B_{\ell \mp 1} \cos \chi \cos \phi^{\prime}+\sin \phi^{\prime}\right)+\underset{\sim}{\hat{y}}\left(-B_{\ell \mp 1} \cos \chi \sin \phi^{\prime}+\cos \phi^{\prime}\right)\right. \\
& \left.-\underset{\sim}{\hat{z}}\left(B_{\ell \mp 1} \sin \chi\right)\right\}
\end{aligned}
$$

and

$$
\begin{aligned}
\Gamma^{-}= & e^{i \ell \pi} \rho A_{\ell \mp 1} \int_{0}^{2 \pi} d X^{\prime}\left(R_{0}-\rho \sin X^{\prime}\right) \int_{0}^{2 \pi} d \phi^{\prime} \\
& \times\left\{\operatorname { e x p } \left\{+i k_{2} \rho \sin \theta^{-} \cos \chi^{\prime}+i k_{2} \cos \theta^{-}\left(R_{0}-\rho \sin X^{\prime}\right) \sin \phi^{\prime}\right.\right. \\
& \left.-i m \phi^{\prime}-i \ell \chi^{\prime}\right\}\left(\hat{x}\left(-B_{\ell \mp 1} \cos X^{\prime} \cos \phi^{\prime}-\sin \phi^{\prime}\right)\right. \\
& \left.\left.+\underset{\sim}{\hat{y}}\left(-B_{\ell \mp 1} \cos \chi^{\prime} \sin \phi^{\prime}+\cos \phi^{\prime}\right)+\hat{z}\left(B_{\ell \mp 1} \sin \chi^{\prime}\right)\right)\right\}
\end{aligned}
$$

and

$$
\Phi^{ \pm}=\left|\Gamma_{\sim}^{ \pm} \cdot\left(\underset{\sim}{\hat{x}} \pm i\left(\underset{\sim}{\hat{y}} \sin \theta^{ \pm}-\underset{\sim}{\hat{z}} \cos \theta^{ \pm}\right)\right)\right|
$$

From an inspection of equations (4) and (5)

and

$$
\begin{aligned}
& \stackrel{\Gamma}{+}^{+} \cdot \underset{\underline{x}}{\hat{x}}=(-1)^{l} \underset{\Gamma^{-}}{\Gamma^{\hat{x}}} \\
& \stackrel{\Gamma}{\Gamma}^{+} \cdot \hat{z}=(-1)^{\ell} \underset{\sim}{\Gamma^{-}} \cdot \underset{\sim}{\underline{z}} \\
& \left.\Gamma^{+} \cdot \underset{\sim}{\tilde{z}}=(-1)^{\ell} \underset{\sim}{\Gamma^{-}} \cdot \underset{\sim}{z}\right] .
\end{aligned}
$$


Thus

$$
\Phi^{+}=\Phi^{-}
$$

and therefore the magnitude of the radiation from each of the azimuthal travelling wave modes is equal but their radiation directions are not. From an inspection of the angular transformations above, we can see that their radiation patterns are mirror images of each other in the plane of the bend, $\theta=\pi / 2$ from fig. 5 . 


\section{REFERENCES}

[1] A.W. Snyder: "Leaky ray theory of optical waveguides of circular cross section", Appl. Phys. 4, 273 (1974).

[2] D. Marcuse: Theory of Dielectric Optical Waveguides (Academic Press, New York, 1974).

[3] R.A. Abram and C.J. Rees: "Mode conversion in an imperfect waveguide", J. Phys. A 6 , 1693 (1973).

[4] E.A.J. Marcatili: "Bends in optical dielectric guides", B.S.T.J. $\underline{48}, 2103$ (1969).

[5] E.A.J. Marcatili: "Dielectric guide with curved axis and truncated parabolic index", B.S.T.J. 49,1645 (1970).

[6] V.V. Schevchenko: "Radiation losses in bent waveguides for surface waves", Izv. VUZ Radiofizika 14, 768 (1971).

[7] L. Lewin: "Local form of the radiation condition: Application to curved dielectric structures", Electron. Letters 9 , 468 (October 1973).

[8] (a) L. Lewin: "Radiation from curved dielectric slabs and fibers", I.E.E.E. Trans. MTT-22, 718 (1974).

(b) L. Lewin: "Correction to 'Radiation from curved dielectric slabs and fibers'", I.E.E.E. Trans. MTT-23, 779 (1975).

[9] J.A. Arnaud: "Transverse coupling in fiber optics. Part III. Bending losses", B.S.T.J. 53, 1379 (1974).

[10] J.A. Arnaud: "Comments on 'Radiation from curved dielectric slabs and fibers'", I.E.E.E. Trans. MTT-23, 935 (1975).

[11] A.W. Snyder, I.A. White and D.J. Mitchell: "Radiation from bent optical waveguides", Electron. Letters 11, 332 (1975).

[12] D. Marcuse: "Curvature loss formula for optical fibers", J. Opt. Soc. Am. 66, 216 (1976).

[13] D. Marcuse: "Field deformation and loss caused by curvature of optical fibers", J. Opt. Soc. Am. 66, 311 (1976). 
[14] D. Marcuse: "Radiation loss of a helically deformed optical fiber", J. Opt. Soc: Am. 66, 1025 (1976).

[15] D.C. Chang and E.F. Kuester: (a) "General theory of surface wave propagation on a curved optical waveguide of arbitrary cross section", Scientific Report No. 11, Electromagnetics Lab., Dept. of Electrical Eng., Univ. of Colorado, Boulder, Colorado.

(b) Supplement to Scientific Report No. 11 (February 1976).

[16] E.F. Kuester and D.C. Chang: "Surface wave radiation loss from curved dielectric slabs and fibers", J. Quant. Electron. QE-11, 903 (1975).

[17] L. Lewin: "Polarisation degeneracy in a bent optical fiber", Electron. Letters 12, 469 (1976).

[18] D. Gloge: "Bending losses in multimode fibers with graded and ungraded core-index", Appl. Optics 11, 2506 (1972).

[19] A.W. Snyder and D.J. Mitchell: "Generalised Fresnel's law for determining radiation loss from optical waveguides and curved dielectric structures", Optik 40, 438 (1974).

[20] D. Gloge: "Propagation effects in optical fibers", I.E.E.E. Trans. MTT-23, 106 (1975).

[21] E.A.J. Marcatili and S.E. Miller: "Improved relations describing directional control in electromagnetic wave guidance", B.S.T.J. 48, 2161 (1969).

[22] S.J. Maurer and L.B. Felsen: "Ray methods for trapped and slightly leaky modes in multilayered or multiwave regions", I.E.E.E. Trans. MTT-18, 584 (1970).

[23] D. Marcuse: (a) "Bending losses of the asymmetric slab waveguide", B.S.T.J. 50, 2551 (1971).

(b) "Bent optical waveguide with lossy jacket", B.S.T.J. 53, 1079 (1974).

[24] S. Kawakami, M. Miyagi and S. Nisheda: "Bending losses of dielectric slab optical waveguide with double or multiple claddings: Theory", Appl. Optics 14, 2588 (1975).

[25] D. Marcuse: Light Transmission Optics (Van Nostrand Reinhold, New York, 1972).

[26] D.C. Chang and F.S. Barnes: "Reduction of radiation loss in a curved dielectric slab waveguide", Sci. Rept. No. 2, Dept. of Elect. Eng., Univ. of Colorado, Boulder, Colorado.

[27] E.G. Neumann and H.D. Rudolf: "Radiation from bends in dielectric rod transmission lines", I.E.E.E. Trans. MTT-23, 142 (1975). 
[28] D.C. Chang and E.F. Kuester: "Radiation of a surface wave from a curvature discontinuity in an impedance surface: Part I. Convex bend", Scientific Rept. No. 16, Dept. of Elect. Eng., Univ. of Colorado, Boulder, Colorado (1976).

[29] S.W. Maley: "Radiation from a circular bend between two discontinuities in a dielectric slab waveguide", Scientific Rept. No. 10, Dept. of Elect. Eng., Univ. of Colorado, Boulder, Colorado (1974).

[30] J.F. Taylor: "Power loss at directional change in dielectric waveguides", Appl. Optics 13, 642 (1974).

[31] D.S. Jones: Theory of Electromagnetism (Pergamon, Oxford, 1964), pp. 562-564.

[32] J.D. Love: "Quasi-static modes of oscillation of a cold toroidal plasma", J. Plasma Phys. 14, 25 (1975).

[33] R.E. Collin: Field Theory of Guided Waves (McGraw-Hill, New York, 1961).

[34] S.A. Schelkunoff: Electromagnetic Waves (Van Nostrand Reinhold, New York, 1943).

[35] L. Lewin: Theory of Waveguides (Newnes Butterworths, London, 1975).

[36] M. Abramowitz and I.A. Stegun: Handbook of Mathematical Functions (Dover, New York, 1972), Chapter 9.

[37] Ref. 36: Chapter 4.

[38] L.M. Brekhovskikh: Waves in Layered Media (Academic, New York, 1960).

[39] A.W. Snyder and D.J. Mitchell: "Leaky rays on circular optical fibers", J. Opt. Soc. Am. 64, 599 (1974).

[40] G.F. Carrier, M. Krook and C.E. Pearson: Functions of a Complex Variable (McGraw-Hill, New York, 1966).

[41] M. Miyagi and G.L. Yip: "Field deformation and polarisation change in a step index fibre due to bending", Optical and Quant. Electron. 8 , 335 (1976).

[42] W.J. Stewart: Private communication.

[43] J.H. Hannay: "Mode coupling in an elastically deformed optical fibre", Electron. Letters. 12, 173 (1976).

[44] A.W. Snyder and W.R. Young: "Modes on optical waveguides", to be published.

[45] F.B. Kapron et aZ.: "Radiation losses in glass optical waveguides", Appl. Phys. 17, 423 (1970). 


\section{CHAPTER 7 \\ SUMMARY AND CONCLUSIONS}

The motivating force behind this thesis was the desire to develop a simple set of theoretical techniques that could be used to analyse the radiative losses in weakly guiding dielectric waveguides and elucidate the physics of the radiation mechanics. To this end, we have presented the Volume Current Method and the Surface Current Method, with which direct simple calculations of the radiation is possible. As discussed in Chapter 1, we are primarily interested in the modal radiative losses as the classical treatment by Coupled Mode Theory is ideally suited to the bound mode coupling.

It is only for the radiation modes of the dielectric waveguide that the analysis via Coupled Mode Theory becomes exceedingly tedious due to the difficulty in the normalisation and orthogonalisation of these radiation modes. From the state of the field of fibre optics, such analyses of dielectric waveguides should be equally applicable to a wide range of dielectric waveguides. To this end, in Chapter 2, we derived a set of modes for an arbitrary dielectric profile for the core of a weakly guiding waveguide of circular cross section and a uniform infinite cladding, and we use these modes in all analyses of the proceeding chapters.

These modal fields are simple in form and circumvent the difficulties of the conventional modal fields [1] when asymptotic analyses are used. Together with this, the analysis predicts extra degeneracies between the $\mathrm{HE}_{\ell}$ and $\mathrm{EH}_{\ell}$ modes, above the usual $\mathrm{HE}_{\ell}$ and $\mathrm{EH}_{\ell-2}$ degeneracy of weakly guiding dielectric optical fibres. Such additional degeneracies have been reported by numerical methods [2] but further analysis of the solutions of Chapter 2 are necessary before it can be demonstrated that these theoretically predicted degeneracies 
correspond to the observed ones.

In Chapter 3, we presented the Volume Current Method and applied it to the situation of isolated weak dielectric irregularities and structural imperfections in optical fibres. We have showed that the Volume Current Method for these radiative loss processes is equivalent to Coupled Mode Theory if the radiation modes used in the latter are approximated by the free space radiation modes. The analysis of section 3.3 illustrated the validity of these calculations for the diffuse scattering processes, such as Rayleigh scattering, where the radiation is spread over a large solid angle in comparison to the solid angle of acceptance of the fibre, i.e. $2 \pi \theta_{\mathrm{C}}^{2}$. However, for highly directional radiation where the radiation lobes are paraxially directed, the Volume Current Method does not provide an adequate description of the radiative losses.

In Chapter 4 , by the simple introduction of planar and cylindrical wave amplitude reflection coefficients, we showed how to include the effect of the core-cladding dielectric difference, ignored in the Volume Current Method. Simple extensions of this analysis of dielectric optical fibre as demonstrated by the general applicability of the cylindrical electromagnetic wave reflection coefficient of Appendix 4.C. This analysis also has the ability to include the effect of the multiple claddings of the practical optical fibre.

In Chapter 5, the philosophy of the Surface Current Method, the vector equivalent of Huygen's Principle, was presented and applied to the weakly guiding dielectric optical waveguide. By the utilisation of the azimuthal travelling wave modes of the circular cylindrical waveguide, the formulae of the method were much simplified. To demonstrate the effectiveness of this approach for the calculation of curvature induced radiation, we applied this simplified Surface Current Integral to calculate the modal power attenuation coefficients of the tunnelling leaky modes of the weakly guiding optical fibre. From this analysis, we found that the only difference between the results for different dielectric profiles of the core, was the power normalisation coefficient ${ }^{\mathrm{A}}{ }_{\ell \mp 1} \cdot$ 
Using this Surface Current Method, in Chapter 6, we analysed the radiative losses from modes of the straight ideal waveguide indicent on a curved segment of waveguide with constant radius of curvature $R_{0}$. By the application of the generalised Fresnel's law for rays incident on a curved interface, we clarified the physical nature of the axial phase matching approximation of the analysis for the bent symmetric slab waveguide, section 6.3. From the ray optical analyses, we derived order of magnitude estimates of the aspect ratio of the bend, $R_{0} / p$, for which we could be justified in assuming that no field deformation nor significant mode coupling occurs, equations $(6.5 .4)$ and (6.5.19).

From our investigation of the bending losses together with the restrictions on the aspect ratio of the bend, we demonstrated that the conventional modal power attenuation coefficients, ignoring all field deformation, were highly accurate over their range of aspect ratios defined by equation (6.4.19) for the $\mathrm{V}=2.4$ discussed. The questions as to the validity of the analyses of bending losses in multimode optical waveguides, employing the phase match along the path of the "centre of energy" of the incident mode, appear to be one of the major unanswered problems of these bending loss studies.

Together with this, the effect of ellipticity of the optical waveguide and the elastic deformation of the waveguide appear not to have been answered successfully to this time. Further analysis as to the effect of non-planar bends and the apparent difference between the radiation losses of the two azimuthal travelling wave modes, is also required.

In the analysis of all six chapters, we have confined ourselves to the quantitative results of the ideal two region fibre. For the practical optical fibre discussed in section 1.3, we must generalise these analyses to multiply clad optical waveguides. Most of the analyses are easily generalised to include the effect of a multiply clad optical waveguide. These generalisations are straightforward and should prove to be extremely accurate. 


\section{REFERENCES}

[1] C.N. Kurtz and W. Streifer: "Guided waves in inhomogeneous focussing media: Part I. Formulation, solution for quadratic inhomogeneity", I.E.E.E. Trans. MTT-17, 11 (1969).

[2] P.J.B. Clarricoats and K.B. Chan: "Electromagnetic wave propagation along radially inhomogeneous dielectric cylinders", Electron. Letters, October (1970). 